\title{
Metabolic reprogramming and redox perturbations
}

\author{
Citation for published version (APA):
}

Aboushousha, R. (2021). Metabolic reprogramming and redox perturbations: an interplay relevant to asthma. [Doctoral Thesis, Maastricht University]. Gildeprint Drukkerijen. https://doi.org/10.26481/dis.20211208ra

Document status and date:

Published: 01/01/2021

DOI:

10.26481/dis.20211208ra

Document Version:

Publisher's PDF, also known as Version of record

\section{Please check the document version of this publication:}

- A submitted manuscript is the version of the article upon submission and before peer-review. There can be important differences between the submitted version and the official published version of record.

People interested in the research are advised to contact the author for the final version of the publication, or visit the DOI to the publisher's website.

- The final author version and the galley proof are versions of the publication after peer review.

- The final published version features the final layout of the paper including the volume, issue and page numbers.

Link to publication

\footnotetext{
General rights rights.

- You may freely distribute the URL identifying the publication in the public portal. please follow below link for the End User Agreement:

www.umlib.nl/taverne-license

Take down policy

If you believe that this document breaches copyright please contact us at:

repository@maastrichtuniversity.nl

providing details and we will investigate your claim.
}

Copyright and moral rights for the publications made accessible in the public portal are retained by the authors and/or other copyright owners and it is a condition of accessing publications that users recognise and abide by the legal requirements associated with these

- Users may download and print one copy of any publication from the public portal for the purpose of private study or research.

- You may not further distribute the material or use it for any profit-making activity or commercial gain

If the publication is distributed under the terms of Article $25 \mathrm{fa}$ of the Dutch Copyright Act, indicated by the "Taverne" license above, 
Metabolic reprogramming and redox perturbations:

An interplay relevant to asthma

\author{
Reem Aboushousha
}


@ Reem Aboushousha, 2021

Metabolic reprogramming and redox perturbations:

An interplay relevant to asthma.

Layout: Reem Aboushousha

Cover image: Ben Lane

Cover design: Reem Aboushousha

Printed by: Gildeprint, Enschede

ISBN: 9789464193725

The research described in this thesis was performed at the Pathology Department, University of Vermont, USA, All rights are reserved. No parts of this thesis may be reproduced, stored in a retrieval system or transmitted in any form or by any means, without prior permission from the author. 


\section{Metabolic reprogramming and redox perturbations: An interplay relevant to asthma}

\section{Dissertation}

to obtain the degree of Doctor at Maastricht University, on the authority of the Rector Magnificus, Prof.dr. Rianne M. Letschert in accordance with the decision of the Board of Deans, to be defended in public on Wednesday December 8th, 2021, at 13:00 hours

by

Reem Aboushousha 


\section{Promotors}

Prof. Dr. E.F.M. Wouters

Prof. Dr. Y.M.W. Janssen-Heininger, University of Vermont, USA

\section{Co-Promotor}

Dr. N.L. Reynaert

\section{Assessment Committee}

Prof. dr. A. Bast (chair)

Prof. dr. C.G. Schalkwijk

Dr. K. Kampen

Prof. dr. C. Brightling, University of Leicester, United Kingdom

Prof. dr. H.I. Heijink, University Medical Center Groningen 
TABLE OF CONTENTS

\begin{tabular}{|l|c|c|}
\hline Chapter 1 & Introduction & 11 \\
\hline Chapter 2 & Outline of the thesis & 41 \\
\hline Chapter 3 & $\begin{array}{c}\text { IL-1/inhibitory KB kinase } \text {-induced glycolysis } \\
\text { augment epithelial effector function and promote } \\
\text { allergic airways disease }\end{array}$ & 47 \\
\hline Chapter 4 & $\begin{array}{c}\text { Activation of Pyruvate Kinase M2 attenuates } \\
\text { expression of pro-inflammatory mediators in house } \\
\text { dust mite-induced allergic airways disease }\end{array}$ & 91 \\
\hline Chapter 5 & $\begin{array}{c}\text { Glutathionylation chemistry promotes interleukin-1 } \\
\text { beta-mediated glycolytic reprogramming and pro- } \\
\text { inflammatory signaling in lung epithelial cells }\end{array}$ & 119 \\
\hline Chapter 6 & $\begin{array}{c}\text { Glutaredoxin-1 augments glutathione levels via } \\
\text { deglutathionylation of the ovarian tumor } \\
\text { deubiquitinase, OTUB1 and stabilization of system } \\
\text { xc - }\end{array}$ & 155 \\
\hline Chapter 7 & General discussion and future perspectives & 191 \\
\hline Chapter 8 & Summary & 211 \\
\hline Chapter 9 & Impact & 215 \\
\hline & Acknowledgments & 223 \\
\hline & 227 \\
\hline
\end{tabular}


Abbreviation List:

1,3-BPG: 1,3-bisphosphoglycerate

2PG: 2-phosphoglycerate

3PG: 3-phosphoglycerate

ADP: adenosine diphosphate

AERD: aspirin-exacerbated respiratory disease

AHR: airway hyperresponsiveness

AMP: adenosine monophosphate

AMPK: adenosine monophosphate-activated protein kinase

ARE: antioxidant response element

ATF4: activating transcription factor 4

ATP: adenosine triphosphate

BALF: bronchoalveolar lavage fluid

BSO: buthionine sulfoximine

CaMKI: Ca2+/calmodulin-dependent protein kinase I

CaMKII: Ca2+/calmodulin-dependent protein kinase II

CAT: catalase

COPD: chronic obstructive pulmonary disease

CREB: c-AMP-response element binding protein

Cys: cysteine

DCs: dendritic cells

DEM: diethyl maleate

DHAP: dihydroxyacetone phosphate

DP: dipeptidase

DUBs: deubiquitnases

EAATs: excitatory amino acid transporters

F6P: fructose 6-phosphate

FBP: fructose 1,6-bisphosphate

G6P: glucose 6-phosphate

GAPDH: glyceraldehyde 3-phosphate

GCL: glutamate cysteine ligase

GGT: y-glutamyltranspeptidase

GGCT: y-glutamylcyclotransferase 
GLRX: Glutaredoxin

GM-CSF: granulocyte macrophage colony-stimulating factor

GPX: glutathione peroxidase

GR: glutathione reductase

GS: glutathione synthase

GSH: glutathione

GSSG: oxidized form of glutathione

GSTs: glutathione S-transferase

GSTP1: glutathione S-transferase Pi 1

HAT: heteromeric amino acid transporter family

HDM: house dust mite extract

HK: hexokinase

ICS: inhaled corticosteroids

IL1B: interleukin-1B

ILC2: innate lymphoid cells type 2

IPF: idiopathic pulmonary fibrosis

Keap1: Kelch-like ECH-associated protein 1

mTORC2: mechanistic target of rapamycin complex 2

$\mathrm{NAD}^{+}$: nicotinamide adenine dinucleotide

$\mathrm{NADH}$ : nicotinamide adenine dinucleotide reduced

NADPH: nicotinamide adenine dinucleotide phosphate

NF-кB: nuclear factor-кB

NOXs: NADPH oxidases

Nrf2: nuclear factor-erythroid 2-related factor 2

$\mathrm{O}_{2}^{-}$: superoxide

OPLAH: 5-oxoprolinase

OTUB1: ovarian tumor (OTU) deubiquitinase binding protein-1

OVA: ovalbumin

PEP: phosphoenolpyruvate

PFK: phosphofructokinase

PK: pyruvate kinase

PKA: protein kinase $A$

PKC: protein kinase $C$

PPP: pentose phosphate pathway 
PRXs: peroxiredoxins

PSSG: protein S-glutathionylation

R5P: ribose 5-phosphate

RNS: reactive nitrogen species

ROS: reactive oxygen species

SOD: superoxide dismutase

$\mathrm{TBH}$ : tert-butyl hydroquinone

TCA: tricarboxylic acid cycle

TGF- $\beta 1$ : transforming growth factor- $\beta 1$

Th2: T helper type 2

TNFa: tumor necrosis factor $\alpha$

TRXs: thioredoxins

TSLP: thymic stromal lymphopoietin

UPR: unfolded protein response 
CHAPTER 1: INTRODUCTION 


\section{$\underline{1.1 \text { Metabolism and glycolysis }}$}

Metabolism can be broadly defined as the sum of the biochemical reactions and processes taking place within each cell that either produce or consume energy. Cellular metabolism is very complex and can be classified into three main categories; anabolism, catabolism, and waste disposal [1]. Anabolism involves the metabolic pathways that synthesize small molecules or polymerize them into complex macromolecules. Catabolism, on the other hand, includes pathways that degrade molecules and compounds and is often accompanied by energy release. Processes responsible for eliminating the toxic waste produced by the other pathways fall under the waste disposal category [1].

Scientific research progressed greatly in studying some of these different metabolic pathways including glycolysis, tricarboxylic acid (TCA) cycle, respiration, oxidative phosphorylation and others. Multiple studies reported strong links between several disease conditions and metabolic perturbations. These scientific data were also supported by many clinical observations. Cells undergo several metabolic adaptations in order to meet specific needs, such as increased energy demand, or the requirement of building blocks for various organelles [2]. These alterations in cellular metabolism in fact have been described as a cause and/or a contributor to many diseases including cancer [3-5], Alzheimer [6], diabetes [7], chronic obstructive pulmonary disease (COPD) [8] and recently asthma [9-11].

Glycolysis, the metabolic pathway that is used by all the cells for energy generation, has been extensively studied in the last century. The word "glycolysis" is derived from the Greek origin "glykys" that refers to sweet, and "Iysis" which means breakdown. The term then describes the splitting/ breakdown of one glucose molecule into two molecules of pyruvate, the end product of glycolysis. This can occur in presence of oxygen (aerobic), where pyruvate enters the mitochondria, or in its absence (anaerobic), in which pyruvate gets converted into lactate. Importantly, glycolysis is the only known pathway that is able to generate ATP in absence of oxygen or in cells lacking mitochondria. In addition to generating ATP, glycolytic intermediates are able to funnel into and fuel many biosynthetic pathways. Under some circumstances, cells consume more glucose and secrete more lactate, even under aerobic conditions. This type of metabolic reprogramming is known as the Warburg effect [3]. The advantage of this type of metabolic reprogramming is not only the generation of copious amount of ATP, but also in the biosynthetic capacity of glycolysis as mentioned above. This enhanced glycolysis was originally described in, and is considered the hallmark of, cancer metabolism, however it is now known to contribute to pathogenesis of multiple inflammatory and metabolic diseases [12, 13]. 
The glycolysis pathway comprises ten steps reactions occurring in the cytosol and generates two molecules of adenosine triphosphate (ATP) without the requirement of oxygen. The pathway can be subdivided into two phases: ATP investment and ATP payoff phases (Figure 1). In the first step of glycolysis, a phosphate group is transferred from ATP to glucose forming glucose 6-phosphate (G6P) in presence of hexokinase enzyme. The G6P then is rearranged into fructose 6-phosphate (F6P) by the action of phosphoglucose isomerase. It is also worthy to note that fructose can enter the glycolysis pathway by phosphorylation at this step [14]. Phosphofructokinase-1 enzyme then converts F6P into fructose 1,6-bisphosphate (FBP) at the expense of one ATP molecule. The FBP's hexose ring then gets split by the enzyme aldolase to form two triose sugars, glyceraldehyde 3-phosphate (GAPDH) and dihydroxyacetone phosphate (DHAP) in the fourth step of glycolysis. The DHAP gets rapidly converted to GAPDH by the action of triose phosphate isomerase enzyme in the fifth and last step of the ATP investment phase of glycolysis. With that said, each molecule of glucose entering the glycolysis pathway generates 2 molecules of GAPDH at the expense of two molecules of ATP in the ATP investment phase of glycolysis (Figure 1). The second payoff phase of glycolysis starts by forming 1,3bisphosphoglycerate (1,3-BPG) from GAPDH through glyceraldehyde 3-phosphate hydrogenase enzyme while utilizing two inorganic phosphate and reducing two molecules of $\mathrm{NAD}^{+}$to $\mathrm{NADH}_{\text {. }}$

In the next step, two ATP molecules are released after the transfer of a phosphate group from 1,3BPG to ADP by phosphoglycerate kinase enzyme forming 3-phosphoglycerate (3PG). The 3PG will be converted to 2-phosphoglycerate (2PG) then to phosphoenolpyruvate (PEP) in the subsequent steps through the action of phosphoglycerate mutase and enolase respectively. The last and final step of glycolysis is catalyzed by pyruvate kinase in which PEP forms pyruvate that can either be converted to lactate or enter mitochondria where it fuels the TCA cycle (Figure 1). The net gain of glycolysis is the generation of two ATP and two NADH molecules from each glucose molecule breakdown. There are three key irreversible steps in the glycolysis pathway (step 1, 3 and 10). These steps are catalyzed by hexokinase, phosphofructokinase and pyruvate kinase. The activity of these three enzymes is crucial for controlling glycolysis and there are multiple ways of regulating them. Allosteric modulators, product inhibition and post translational modifications are examples of the different mechanisms that contribute to the activity of these three key enzymes [15-17]. 


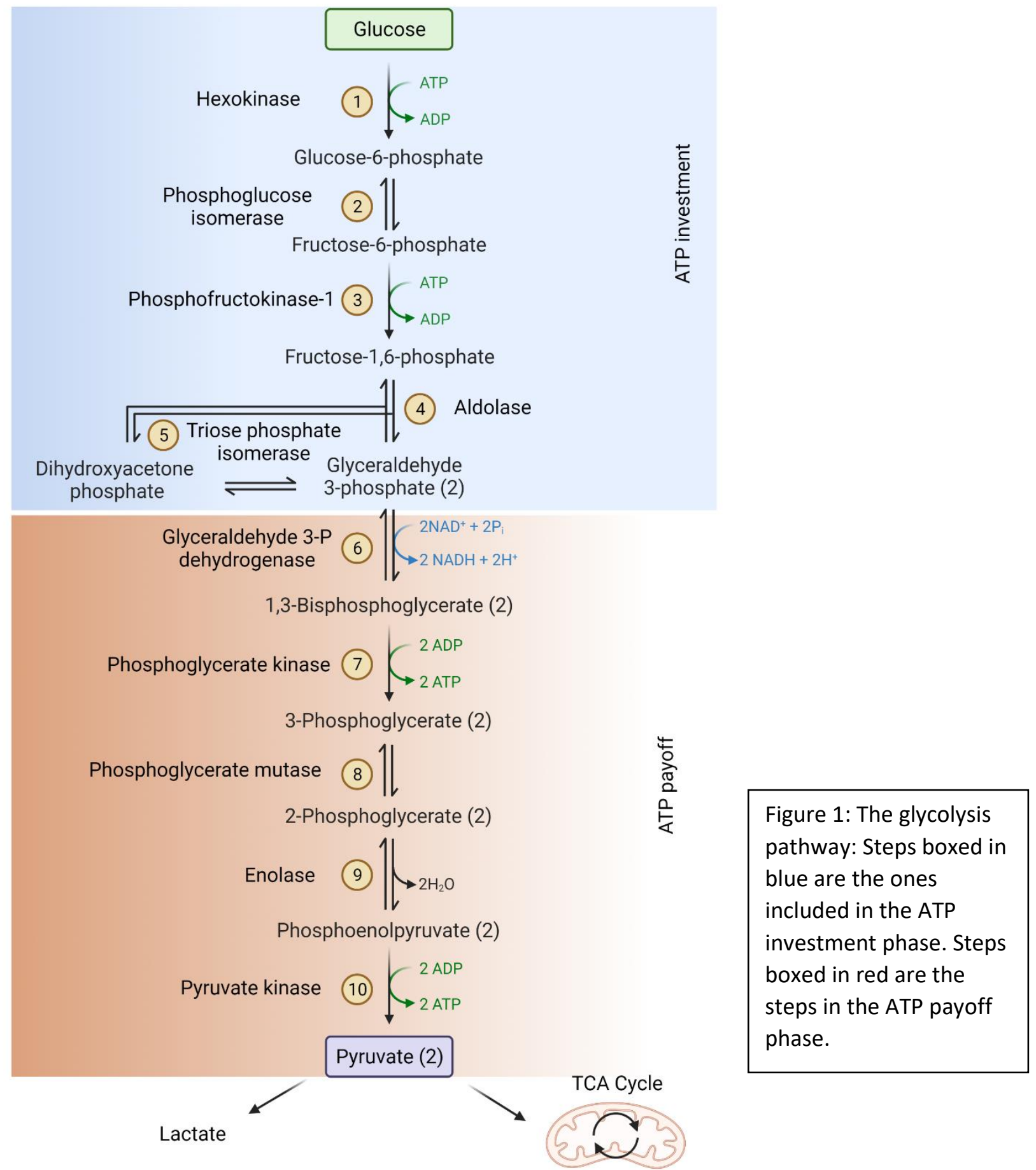

Hexokinase (HK), the enzyme that catalyzes the conversion of glucose to G6P in the first step of glycolysis, plays a major role in determining the rate of the pathway. HK has four subtypes: HK-I, II, III and IV. HK-I is the main isoform found in brain, but in addition it is also ubiquitously expressed in other tissues. HK-I is considered, in most cases, as housekeeping enzyme that is 
not affected by most metabolic changes. HK-Il however is the most regulated isoform of all hexokinases. It is predominantly found in insulin sensitive tissues such as cardiac muscles, and it is upregulated in many cancers. HK-III is universally expressed without dominating any tissues, but little is known about this isoform and its regulation. The fourth isoform HK-IV (also known as glucokinase) is mainly found in liver and pancreas. This isoform differs in its kinetics and function than the other three HK isoforms. HK-IV has a Km for glucose that is 100 times higher than HKI, -II and -III, which means it will only phosphorylate glucose if its concentration is very high [1821].

Phosphofructokinase (PFK) is considered the most crucial and the rate limiting enzyme in the glycolysis pathway [22]. PFK is subjected to extensive regulation since it catalyzes the committed step in the glycolysis pathway (phosphorylation of F6P into FBP). PFK can undergo allosteric inhibition by high levels of ATP, an effect that can be reversed by AMP. In this type of regulation, a lower ATP/AMP ratio increases PFK activity and enhances glycolysis [23]. Low $\mathrm{pH}$ levels, such as in the case during excessive lactic acid release from muscles after strenuous exercise, also inhibits PFK and hence protect the muscle from extensive damage [24]. PEP, acylcoA, citrate and ADP have also been reported to allosterically inhibit PFK [25, 26], while fructose 2,6-bisphosphate leads to its activation [27]. Cancer cells as well as some viruses including the herpes simplex virus type-I over express and/or activate PFK enzyme to keep up with their high metabolic demand [27, 28].

Pyruvate kinase (PK), the enzyme that catalyzes the last and irreversible step of glycolysis, is another key regulator of the pathway. There are four mammalian PK isoforms, PKM1, PKM2, PKL and PKR. PKM1 is present in the tetrameric form and is expressed in tissues with high catabolic demand such as brain, heart, and muscles [29]. PKM2 is highly expressed during embryogenesis and is present in proliferating cells [30]. PKM2 presents itself in a monomer, dimer, or tetramer form, with each of these structures dictating a different intracellular function. PKL isoform is found mainly in the liver and to lower extent in kidneys, while PKR is exclusive to red blood cells [31, 32]. As expected, the pyruvate kinase enzyme undergoes extensive regulation. FBP binds to the allosteric binding site on domain $C$ of PK, changing its conformation and leading to its activation [33, 34]. Serine is another allosteric activator of PKM [35, 36], while phenylalanine, alanine, ATP, and thyroid hormone T3 can all inhibit the PK enzymatic activity [37-39]. Post translational modifications also play a key role in PK regulation. Phosphorylation of the PKM2 isoform at tyrosine 105 has been described to suppress its activity [40]. In addition, higher ROS concentration during oxidative stress inhibits PKM2 activity through oxidizing the enzyme at cysteine 358 [41]. Acetylation at lysine 305 is another example of post 
translational modification of PKM2 that leads to its degradation [42]. Other types of modifications that can regulate PKM include methylation [43], ubiquitination [44], hydroxylation [45], glycosylation [46] and sumoylation [47]. Given the extensive regulation of pyruvate kinase, it is no wonder that any dysregulation and/or modification to its structure will have drastic effects on its fate and activity.

\subsection{Glycolysis and redox homeostasis}

Glycolysis and glycolytic reprogramming have been linked to redox homeostasis through the pentose phosphate pathway (PPP). The PPP is an offshoot of glycolysis that starts with the consumption of G6P and generates F6P and GAPDH. In addition, the PPP supplies ribose 5phosphate (R5P) and NADPH, instead of ATP in case of glycolysis. The R5P is the building block for nucleic acid synthesis, while the NADPH is the major reducing equivalent that drives biosynthesis of fatty acids, cholesterol, amino acids, and nucleotides, in addition to its indispensable role in redox homeostasis [48] (Figure 2).

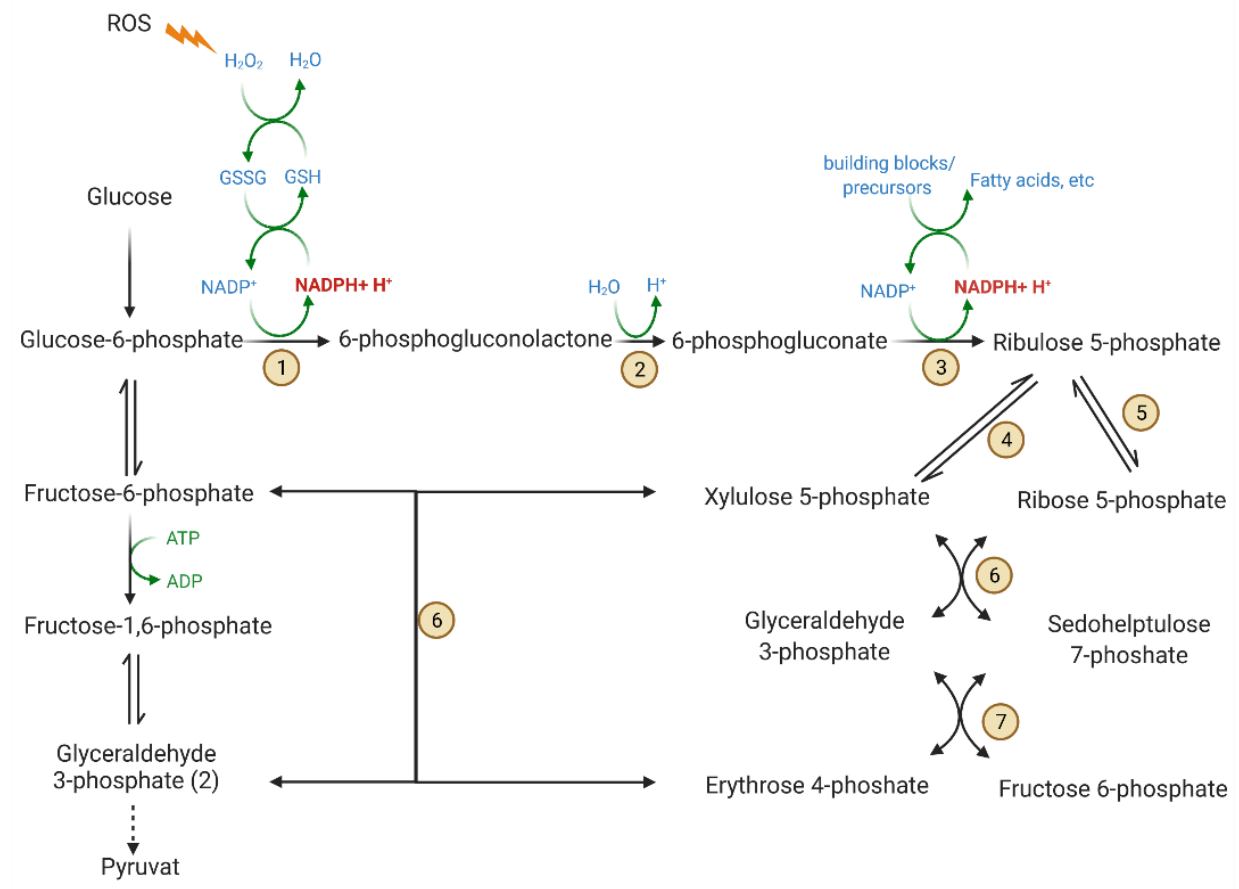

The term "redox homeostasis" has 2 components: "redox" which is used as an abbreviation for reduction/oxidation, and "homeostasis" that describes the balance between the reactive oxygen/nitrogen species
Figure 2: The pentose phosphate pathway (PPP): PPP branches from glycolysis at the glucose 6phoshate step before it gets back at the fructose 6-phosphate and glyceraldehyde 3phosphate steps. PPP generates NADPH necessary for redox homeostasis. 1: Glucose 6phosphate dehydrogenase, 2: 6phosphoglucololactonase, 3: 6-phosphogluconate dehydrogenase, 4 : ribulose-5-phosphate epimerase, 5: Ribose-5phosphate isomerase, 6 : transketolase, 7 : transaldolase (ROS/RNS) production and removal. NADPH generated from the PPP, is used in the regeneration of GSH antioxidant (discussed below) following oxidants scavenging and in the generation of 
superoxide $\left(\mathrm{O}_{2}^{-}\right)$by NADPH oxidases (NOXs), therefore is essential for maintaining redox balance $[49,50]$.

Reactive oxygen species (ROS) are usually generated as byproducts of normal physiological processes. Contrary to the notion that oxidants are always bad, moderate amounts of ROS are indeed beneficial in many cases such as in wound healing and fighting invading pathogens [51, 52]. ROS are also well described as key signaling regulators governing autophagy, necrosis, apoptosis, proliferation, as well as other cellular processes [53-58]. ROS elicit their effects altering protein's structure and function through special types of oxidations on reactive cysteine residues of their target protein. Methionine, tyrosine and tryptophan are also targets for oxidation by ROS, however they are not as well studied and will not be focused on here. Reactive cysteine can be oxidized to form sulfenic or sulfinic acids $\left(-\mathrm{SOH},-\mathrm{SO}_{2} \mathrm{H}\right)$, form a disulfide with another cysteine (S-S), gets $\mathrm{S}$-nitrosylated if there is a nitrogen nearby (-SNO), forms a protein mixed di-sulfide with glutathione (PSSG) or gets irreversibly oxidized to sulfonic acid (SO3H) [59] (Figure 3).

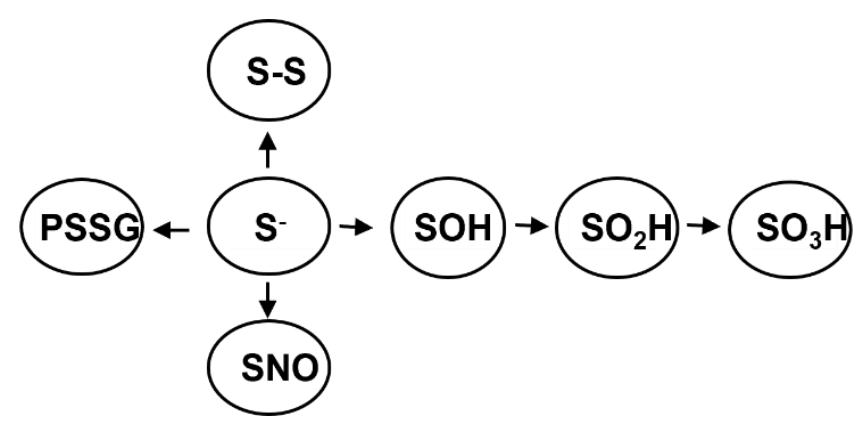

Figure 3: Different fates of cysteine oxidation: $S$ : reactive cysteine, PSSG: S-glutathionylation, S-S: disulfide, SNO: S-nitrosylation, $\mathrm{SOH}$ : sulfenic acid, $\mathrm{SO}_{2} \mathrm{H}$ : sulfinic acid, $\mathrm{SO}_{3} \mathrm{H}$ : sulfonic acid. Arrows serve illustrative purposes only and do not indicate directionality. Adapted from [59]

Several antioxidant systems are responsible for eliminating excessive amount of ROS in order to maintain the redox homeostasis. They can be subcategorized to enzymatic and nonenzymatic antioxidants. The enzymatic category include: thioredoxins (TRXs), glutathione Stransferase (GSTs), catalase (CAT), superoxide dismutase (SOD), glutathione peroxidase (GPxs) and peroxiredoxin (PRXs). In addition, cells also use non-enzymatic small antioxidant molecules, such as glutathione (GSH), ascorbic acid (vitamin C), $\alpha$-tocopherol (Vitamin E) and $\beta$-carotene to neutralize oxidants and fight oxidative stress. The focus of this thesis will be on the antioxidant glutathione.

\subsection{Glutathione}


The most abundant non-protein thiol antioxidant that is present in all mammalian cells is glutathione (GSH). The antioxidant is a tripeptide composed of cysteine, glycine and glutamic acid. GSH can exist in the thiol-reduced (GSH) or thiol-oxidized (GSSG) forms, with GSH being the predominant form and is present in millimolar concentration in the cytosol, while GSSG represents less than $1 \%$ of total GSH [60]. Glutathione reductase (GR) is the enzyme responsible for catalyzing the reduction of GSSG to GSH, using NADPH as a cofactor, thereby maintaining GSH homeostasis. There are two ATP requiring steps involved in GSH synthesis in the cytosol:

1- $L-$ Glutamate + L cysteine $+A T P \rightarrow \gamma-$ glutamyl $-L-$ Cysteine $+A D P+P i$

2- $\gamma-$ glutamyl $-L-$ Cysteine $+L-$ glycine $+A T P \rightarrow G S H+A D P+P i$

The first step is catalyzed by glutamate cysteine ligase (GCL) and is considered the rate limiting step in GSH synthesis, while the second step is catalyzed by glutathione synthase (GS). GCL is composed of two subunits: the catalytic or heavy subunit GCLC and a modifier or light subunit GCLM. GCLC subunit exhibits all the catalytic activity of the enzyme and is subjected to feedback inhibition by GSH $[61,62]$. On the other hand, GCLM subunit lowers the Km of GCL for glutamate and raises the Ki for GSH, making the holoenzyme more catalytically efficient and less subject to inhibition by GSH than GCLC alone [62]. In addition to GCL and GS, several other enzymes are involved in the synthesis and/or degradation of GSH including: $\gamma$-glutamyltranspeptidase (GGT), V-glutamylcyclotransferase (GGCT), 5-oxoprolinase (OPLAH), and dipeptidase (DP) (Figure 4). The enzyme GGT breaks down GSH extracellularly into cysteinylglycine and $\gamma$-glutamyl aminoacid. Cysteinylglycine gets broken down into cysteine and glycine by the DP enzyme, where they can be re-uptaken by the cells and incorporated in GSH synthesis or shunted into other metabolic pathways. The $\gamma$-glutamyl aminoacid will be converted to 5-oxoproline, by the action of GGCT, before it gets converted back to glutamate by OPLAH enzyme and then used in GSH biosynthesis [63-65]. Recently, exciting discoveries have identified the glutathione-specific $\mathrm{Y}^{-}$ glutamylcyclotransferase $\mathrm{ChaC} 1$ and 2 as another GSH degradation pathway that takes place intracellularly [66, 67]. ChaC1 was originally identified downstream of activating transcription factor 4 (ATF4) as part of the unfolded protein response (UPR) before proving its involvement in other processes such as proliferation and apoptosis [68-70]. ChaC1, and to a lesser extent ChaC2, break down intracellular GSH into cysteinylglycine and 5-oxoproline directly [66, 67]. 


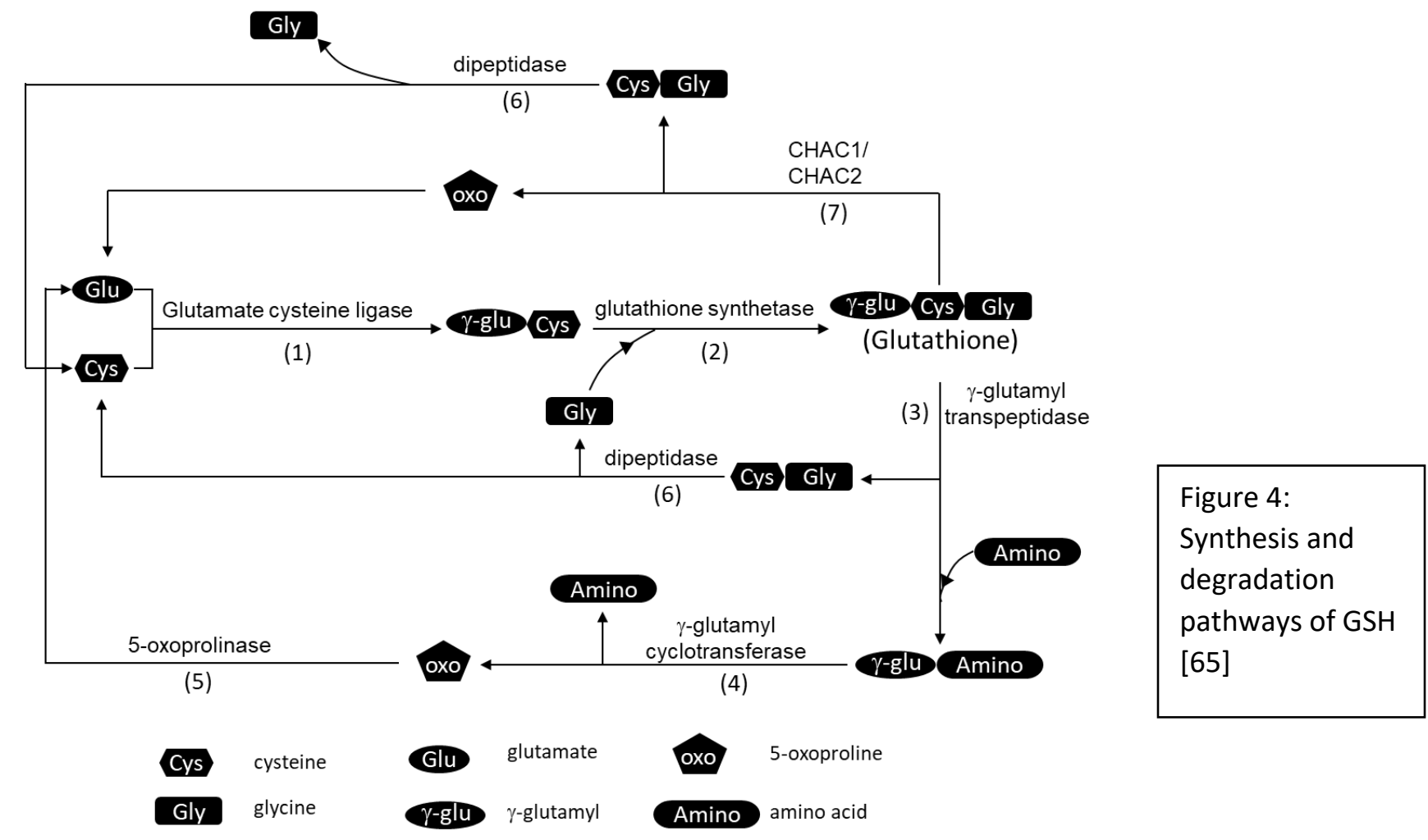

In addition to its function as an antioxidant, GSH plays a role in several other vital processes in our bodies. For example, the elimination of several xenobiotics is accomplished by conjugation with GSH followed by the secretion of the glutathione adduct from the cells through membrane transporters such as the multidrug resistant proteins [71]. GSH also regulates cell cycle progression [72], and its level correlates with growth of many cell types including liver and metastatic cancer melanoma cells [73]. The $\gamma$-glutamyl cycle proves that GSH also serves as a continuous source of cysteine, which is essential for protein and non-protein compounds biosynthesis [64, 74]. Metabolism of estrogens, leukotrienes, and prostaglandins, synthesis of DNA and maintenance of the cell redox potential are also among the several functions of GSH [75-79].

\subsection{Glutathione and redox signaling}

One critical role of GSH is modulating redox-dependent cell signaling. Protein Sglutathionylation (PSSG), which occurs through the conjugation of GSH to a protein cysteine residue, is an important regulator of multiple biological processes due to its effect on changing proteins' structure and/ or function (Figure 5). For instance, S-glutathionylation of the cytoskeletal protein actin inhibits its polymerization $[80,81]$. Aldolase S-glutathionylation, is another example 
in which S-glutathionylation regulates cellular functions through inhibiting the enzymatic function of the S-glutathionylated target [82]. Uncoupling proteins UCP2 and UCP3, mitochondrial complex I and $\mathrm{Ca} 2+/$ calmodulin-dependent protein kinase I (CaMKI) are some other examples of targets inhibited by S-glutathionylation [83-85], while the $20 \mathrm{~S}$ proteasome core and adenosine monophosphate-activated protein kinase (AMPK) exhibit increases in their activities following Sglutathionylation $[86,87]$.

The post-translational modification S-glutathionylation is a reversible process. While glutathione S-transferase Pi 1 (GSTP1) catalyzes the forward reaction leading to PSSG of some proteins, the thiol transferase glutaredoxin (GLRX) is responsible for the reverse reaction and removal of GSH (Figure 4). Interestingly, GLRX has emerged as a key regulator of multiple physiological processes including inflammation, energy metabolism and fibrosis [88-90].

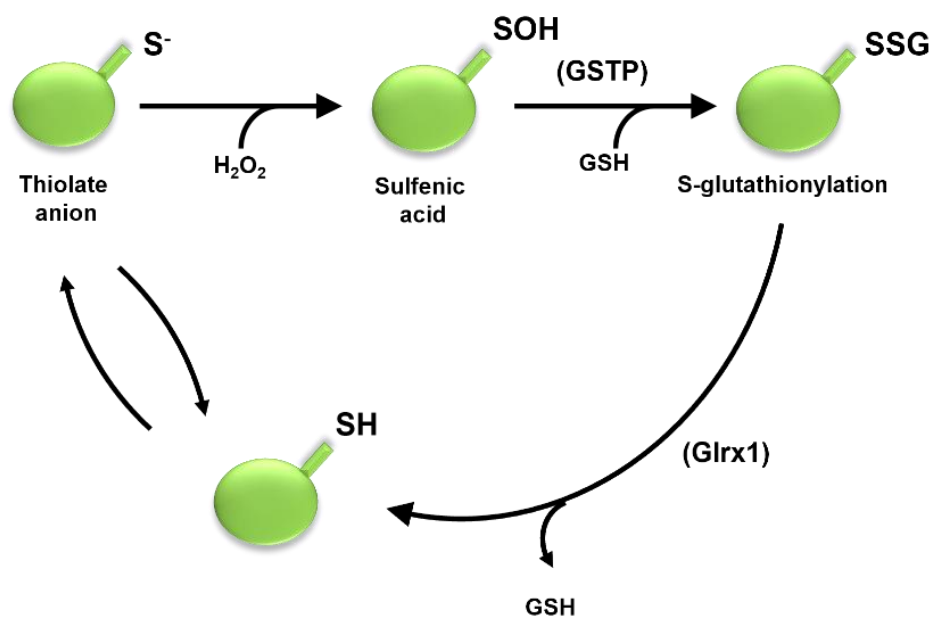

Figure 5: Overview of the Sglutathionylation process: GSTP: glutathione S-transferase Pi 1. Glrx1: Glutaredoxin 1

\subsection{Glutathione regulation}

Given the vast cellular processes influenced by GSH, it is not surprising that it is extensively regulated. In addition to GSH recycling from GSSG by the GR, at the expense of NADPH (mentioned above), regulation of cellular GSH levels falls under two main mechanisms: regulation of the synthesis rate and regulation of its export from cells. The rate of GSH synthesis is largely controlled by the expression and the catalytic activity of $\mathrm{GCL}$, which catalyzes the first step in GSH biosynthesis. As discussed above, GCLC subunit can be regulated through a feedback inhibition by GSH, that is attenuated when GCLM subunit is present [62]. Oxidants and electrophiles however have been shown to increase expression levels of GCLC and GCLM subunits [91, 92]. The Nuclear factor-erythroid 2-related factor 2 (Nrf2) is a key transcription factor that is kept in the cytosol by Kelch-like ECH-associated protein 1 (Keap1), under physiological conditions. Upon oxidative stress, Nrf2 dissociates from Keap1 and translocates to the nucleus where it upregulates expression of genes involved in the antioxidant defense, including GCLC 
and GCLM [93, 94]. The nuclear factor-kB (NF-kB) is another regulator of GSH that has been described to control basal levels of GCLC and GCLM subunits [95]. Moreover, under oxidative stress, the transcription factor c-Myc binds to GCLC and GCLM promoters and induce their activities [96]. In addition, other transcription factors such as C-AMP-response element binding protein (CREB) and transforming growth factor- $\beta 1$ (TGF- $\beta 1$ ) have also been described to regulate GCLC expression [97-99]. Post-translationally, GCLC is a target for phosphorylation by protein kinase $A(P K A)$, protein kinase $C(P K C)$ or Ca2+-calmodulin kinase II (CaMKII), and its phosphorylation/ dephosphorylation status impact its activity [100]. GCLC subunit also undergoes caspase-3 dependent cleavage which inhibits its activity [101]. Although studied with little attention, GS, the enzyme catalyzing the second step in GSH synthesis, can also be regulated by Nrf2 [102]. Interestingly, several treatments such as diethyl maleate (DEM), buthionine sulfoximine (BSO), tert-butyl hydroquinone (TBH), tumor necrosis factor $\alpha$ (TNFa) and hepatocyte growth factor (HGF) were reported to induce expression of the two GCL subunits as well as GS enzyme [72, 103].

The second major regulator of GSH synthesis is the cysteine availability. Diet, protein breakdown and the transsulfuration pathway are all sources of cysteine intracellularly [104]. Extracellularly, cysteine is found in its oxidized "disulfide" form (cystine), that gets reduced to two cysteines intracellularly $[105,106]$. For this reason, transporters that allow cystine uptake intracellularly are a great determinant of GSH homeostasis.

\subsection{System $\mathrm{x}_{\mathrm{c}}^{-}$}

System $\mathrm{x}_{c}{ }^{-}$was first discovered in 1980 by Bannai and Kitamura in human fetal lung fibroblasts before it grabbed scientists' attention in the following years [107]. The sodiumindependent, chloride-dependent antiporter is responsible for importing cystine into the cells while exporting glutamate in 1:1 ratio. Once inside the cells, the cystine will be reduced to its monomeric form, cysteine, that can be incorporated in GSH synthesis. For this reason, system $x_{c}^{-}$is considered a key regulator of cellular redox homeostasis. The $x_{c}{ }^{-}$transporter is a member of the heteromeric amino acid transporter (HAT), a family of amino acid transporters consisting of a light and a heavy chain linked by a disulfide bridge [108]. In case of $x_{c}^{-}$, the heterodimer is composed of the light SLC7A11 subunit (also known as $x C T$ ), which is responsible for the transporter activity, linked via a disulfide bridge to SLC3A2 (also known as CD98, 4F2hc) heavy subunit, that traffics the light chain to the cell surface [109, 110] (Figure 6). Along its most obvious role in providing cysteine and maintaining redox homeostasis through GSH synthesis, system $\mathrm{x}_{c}{ }^{-}$also represents a non-vesicular source of extrasynaptic excitatory signal in CNS through the export of glutamate 
[111]. Interestingly, changes in $x_{c}^{-}$activity and subsequently glutamate levels at these synapses have been associated with the pathophysiology of drug addictions [112-115]. In the eyes, $x_{c}^{-}$mediated glutamate release, in combination with glutamate vesicular release, were shown to be important for the signaling through the photoreceptor, further pointing to the diverse roles played

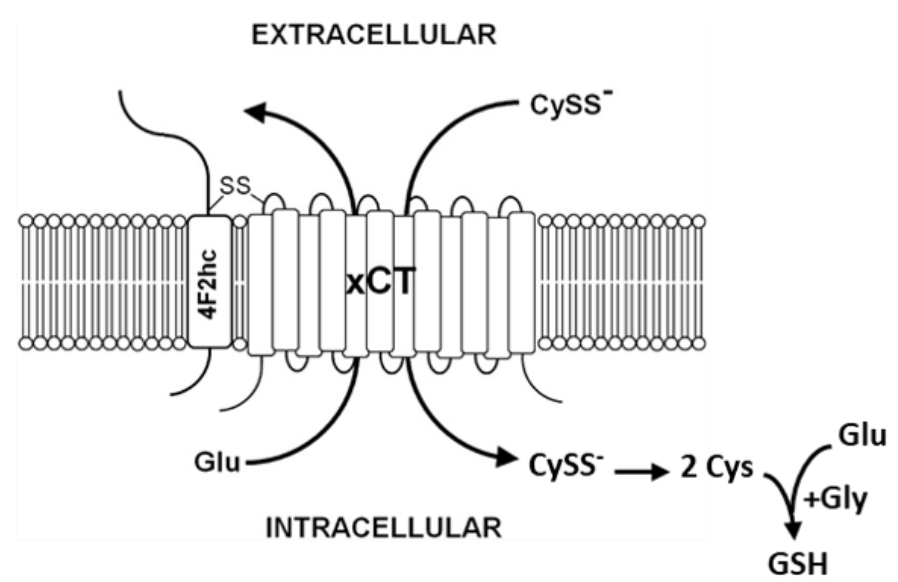

Figure 6: system $x_{c}^{-}$structure and function: CySS-: cystine, Cys: cysteine, Glu: glutamate, Gly: Glycine. Adapted from [110]

by $x_{c}^{-}[116]$. Given the crucial roles played by $x_{c}^{-}$transporter, and the importance of its substrates, it is not surprising that the dysregulation of the transporter has been linked to a variety of diseases. Indeed, Alzheimer's disease [117], Parkinson's disease [118], multiple sclerosis [119] and bacterial infection [120] are among some of the diseases affected by system $x_{c}{ }^{-}$dysregulation. However, the strongest link between overexpression/ overactivation of the $x_{c}^{-}$transporter and a disease has been established in cancer, where $x_{c}{ }^{-}$is necessary for the growth, expansion, and survival of many cancer subtypes [121, 122].

Multiple mechanisms have been identified as regulators of system $x_{c}$. For instance, oxidative stress [123], electrophilic agents [124], amino acid deprivation [125, 126], the proinflammatory cytokine TNF- $\alpha$ [127], as well as exposure to xenobiotics [128] have all been linked to the upregulation of system $x_{c}$. As the case with multiple genes in the GSH biosynthesis pathway, SLC7A11 has multiple antioxidant response element (ARE) motifs in its promoter region of the gene $[123,129,130]$. Nrf2 binds to these ARE regions, following its activation and translocation to the nucleus, leading to the upregulation of these genes [130-132]. A variant isoform of the adhesion molecule CD44 (CD44v), which is expressed in cancer stem cells, has been described as a regulator of system $x_{c}^{-}$, through its interaction with SLC7A11/SLC3A2 subunits, and stabilizing their membrane insertion [133]. Substrates availability was also identified as a regulator of the $x_{c}{ }^{-}$transporter. In this type of regulation, high concentration of extracellular glutamate acts as a competitive inhibitor of the transporter. When needed and in order to overcome this deactivation, cells re-uptake the glutamate via the excitatory amino acid transporters (EAATs) and this in turn activates the cystine import [134-136]. 
Recently, activity of system $x_{c}^{-}$has been shown to be also regulated at the posttranslational level. The mechanistic target of rapamycin complex 2 (mTORC2) was shown to be capable of phosphorylating serine 26 of the cytosolic N terminus of SLC7A11, inhibiting its activity [137]. Protein ubiquitination, which is the post-translational modification targeting proteins for degradation via the proteasome, has also been linked to system $x_{c}^{-}$regulation [138]. The ubiquitination process can be reversed by the action of deubiquitnases (DUBs). Several families and members of DUBs have been identified, with the ovarian tumor (OTU) deubiquitinase binding protein-1 (OTUB1) member being linked to system $x_{c}^{-}$regulation. OTUB1 was found to directly bind to and stabilize SLC7A11 in a CD44-dependent manner in cancer cells [138].

\subsection{Redox homeostasis in the development of lung diseases}

Lungs, beside their continuous contact with oxygen in ambient air, are also exposed to several other exogenous oxidants, such as these accompanying cigarette smoke, air pollutants, irritants, sulfur, nitrogen oxides (NOx) and ozone [139, 140]. In addition, lungs are also exposed to endogenous oxidants generated as byproducts of normal cellular processes or released by macrophages that normally reside along the lungs' epithelial surface. Moreover, neutrophils, eosinophils and leukocytes that are recruited to the epithelia under acute and chronic inflammatory lung disorders also generate ROS that can produce substantial damage [141, 142]. Therefore, lungs have a strong antioxidant capacity to protect themselves from the harmful effects of oxidants and electrophilic molecules. In fact, concentration of the antioxidant GSH in the lung lining fluid, is 100 times higher than that found in blood [143]. Lungs also utilize more GSH than any other organ [144]. Despite this protection, lung damage and diseases still occur, with the imbalance and/or failure of this protective mechanism contributing to pulmonary pathologies. For example, diseases such as idiopathic pulmonary fibrosis (IPF), chronic obstructive pulmonary disease (COPD) and asthma, have been linked to oxidative stress without a clear mechanistic explanation. From these chronic non-communicable respiratory conditions, we will discuss asthma in greater detail.

\subsection{Asthma}

A very common but complex lung disease that affects people of all ages is asthma. With 25 million people affected in the US alone and approximately 300 million people affected worldwide, asthma presents a widespread disease with huge burden on economies [145, 146]. The disease is characterized by chronic airway inflammation, airflow obstruction, airway hyperresponsiveness (AHR) and airway remodeling. Symptoms of asthma include shortness of 
breath, wheezing, chest tightness, cough accompanied with reversible airway obstruction. Several subtypes of asthma have been identified including occupational asthma, steroid-resistant asthma, cold air or exercise-induced asthma as well as the most studied subtype allergic asthma. Additionally, there are several risk factors that are linked to asthma development. These include exposure to environmental allergens such as dust mites, air pollution, tobacco smoking, viral infection, and obesity. Although asthma often begins in childhood, the disease can still occur anytime throughout life. And while asthma prevalence is higher in children, the complications and mortality are a lot more prevalent in adults [146].

\subsection{Pathophysiology of Asthma}

Through the years, asthma has been well described with its hallmarks of reversible airflow obstruction, bronchial hyper-reactivity, and chronic airway inflammation without affecting the lung parenchyma [147]. The underlying mechanism of the chronic airway obstruction and airflow limitation in asthma is complex and includes multiple key players. Allergic asthma has two phases, an early and a late phase. The early phase is initiated by the $\lg \mathrm{E}$, which is produced by plasma cells in response to sensitization to the common inhaled allergens such as, house dust mite (HDM), fungi, animal dander or pollens. Upon exposure to these allergens, IgE binds the high affinity lgE receptor ( $F c \varepsilon R I)$ expressed on mast cells leading to their activation. This in turn leads to the release of several mediators that activate epithelial, endothelial, and smooth muscle cells, neurons as well as other immune cells, thereby inducing changes in lung function [148, 149]. In addition, T helper type 2 (Th2) lymphocytes produce several types of interleukins (IL-) such as IL4, IL-5 and IL-13 as well as the granulocyte macrophage colony-stimulating factor (GM-CSF), which communicate with other cell types to sustain the inflammation [150, 151]. IL-3 and IL-5 help eosinophils and basophils to survive, while IL-13 contributes to remodeling, fibrosis and hyperplasia [150]. All these events lead to the sensitization of the patients to a specific allergen. Re-exposure to the same sensitized allergen, induces an aggregation on receptor-bound $\lg \mathrm{E}$ molecules and results in the activation of type I hypersensitivity reactions [152]. The later phase of the disease typically starts within the next few hours, when dendritic cells (DCs) process the antigen molecules into small peptides and present them, through the major histocompatibility complexes I and II, for recognition by T cell receptors. There are no DCs in the airways at birth, but upon damage to and activation of the respiratory epithelium, bone marrow starts producing immature DCs that will migrate towards the epithelium and underlying mucosa in response to chemoattractants $[153,154]$. The presence of the GM-CSF released from the epithelium and other structure and immune cells in presence of IL-4, and tumor necrosis factor- $\alpha$ (TNF- $\alpha$ ), leads to DC 
maturation to fully competent antigen processing cells. These mature DCs form an immunologic synapse with the allergen-specific T lymphocyte to initiate a Th response. Some of the Th cells will be directed to the $\mathrm{B}$ - cell follicle to facilitate immunoglobulin class switching from $\lg \mathrm{M}$ to $\lg \mathrm{E}$, while others will move back to the airway mucosa and induce the classical Th2 response through secretion of proinflammatory cytokines [155]. Eosinophils, basophiles, neutrophils as well as helper and memory T-cells localize to the lungs in the late phase of asthma and contribute to the inflammation and bronchoconstriction.

Multiple studies suggest that asthma persistence is driven by the ongoing host immune response that generates mediators inducing the airway dysfunction and remodeling. Increased airway smooth muscle mass, thickening of basement membrane and subepithelial fibrosis are all hallmarks of airway remodeling [156] (Figure 7).

\subsection{Airway epithelium}

Airway epithelial cells play a critical role in asthma progression and development. These cells are the first line of defense against inhaled allergens and consist mainly of basal cells that are found in close proximity to ciliated and secretory cells, together forming a tight unit that not only maintains a physical barrier but also responds to the inhaled environment. Ciliated and secretory cells function primarily to facilitate the mucociliary clearance of particulate matter and infectious pathogens in the air we breathe. In addition, and like other mucosal surfaces, the airway epithelium is at an interface with the environment and is therefore critically important to host defense. An impaired epithelial barrier in susceptible individuals, exposes their airways rendering them more vulnerable to virus infections, and penetration by inhaled allergens [157]. Once activated, the airway epithelial cells produce a variety of cytokines, including thymic stromal lymphopoietin (TSLP), GM-CSF and interleukins (IL) -25 and -33, that drive the Th2 response $[74,158-160]$. 


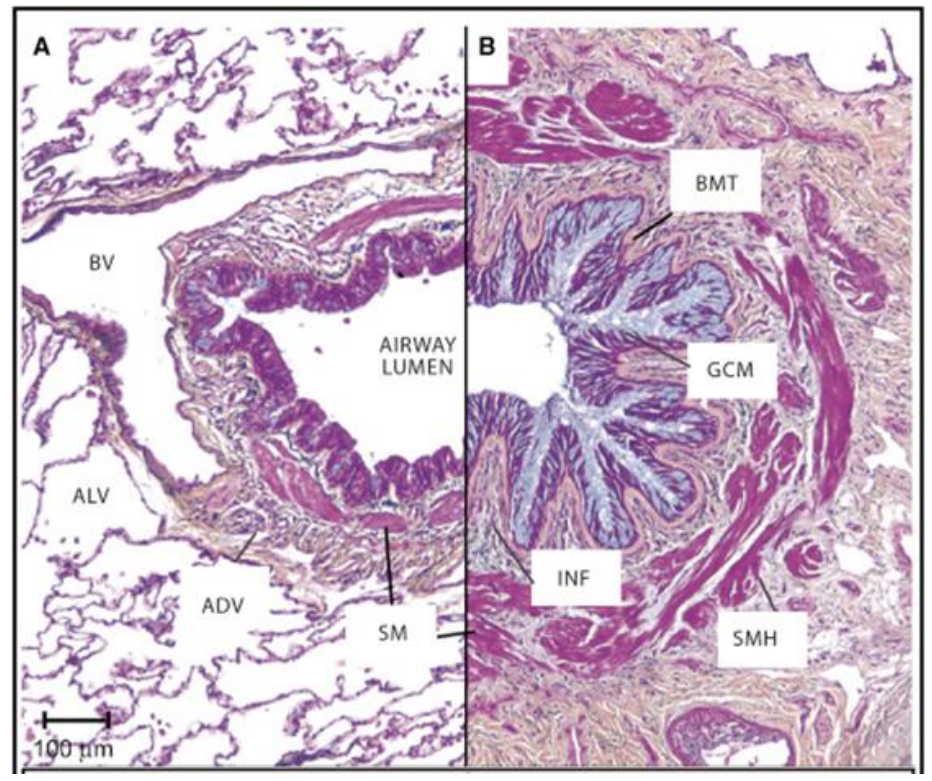

Figure 7: Asthma under the microscope

A: Histology of a healthy control lung. B: Lung of a patient with severe asthma highlighting the airway remodeling, inflammation (INF), basal membrane thickening (BMT), smooth muscle hypertrophy (SMH) and goblet cell metaplasia (GCM) [151].

\subsection{Classification of asthma subtypes}

The complexity of asthma, its heterogeneity, different underlying mechanisms, and different treatment responsiveness, urged for a better classification of asthma subtypes. The original thought that asthma is considered an excessive Th2 response and IgE-driven airway hyperresponsiveness does not apply to all asthma patients [151]. Indeed, the newer classification of asthma, generated by employing different methodologies and data sets in an unbiased way, divides asthma into two subclasses: type 2 (T2) and non-type 2 (non-T2) groups [161-163]. Characteristics of the T2 subgroup include higher levels of airway inflammation, airway eosinophilia and higher numbers of mast cells in airways [164, 165]. T2 endotype can further be subdivided into early-onset "extrinsic" allergic asthma, late-onset "steroid-resistant" eosinophilic asthma and aspirin-exacerbated respiratory disease (AERD) [165]. The non-T2 patients are usually characterized with neutrophilic or pauci-granulocytic (normal levels of eosinophils and neutrophils in sputum) inflammation, resistance to corticosteroids, and absence of T2- biomarkers such as eosinophilia. Unfortunately, the mechanistic etiology of non-T2 subtype is not yet well defined, however, chronic infection with atypical bacteria, smoking and obesity have all been linked to play a role in its disposition [166-168]. Although currently we have better classifications of asthma and its subtypes, a huge overlap between these subcategories exists in subpopulation of patients.

Inhaled corticosteroids (ICS) combined with short acting $\beta$-agonists are the first line treatment for asthmatics, which were proven to reduce asthma exacerbations and improve the 
disease control [169-171]. Patients with poorly controlled asthma and history of exacerbations, however, may benefit more of combining ICS with long acting $\beta$-agonists [172]. Other asthma treatment strategies include leukotriene antagonists [173, 174], and targeted biological therapy [175]. Unfortunately, as mentioned above, there is a subgroup of patients that cannot be classified under one asthma subtype and are not responsive to the conventional therapy. Therefore, more studies are needed to better understand and characterize specific biomarkers to help with treatment optimization and overall care [176].

\subsection{Metabolism and redox homeostasis in asthma}

As previously explained, the aberrant inflammatory response induced by respiratory insults and the airway epithelial damage, creates a state of chronic activation of innate immune effector cells leading to lung remodeling [177]. This activation is usually accompanied by widespread changes in cellular metabolism [178], as well as an imbalance in the redox environment [179-181]. In addition, large number of studies have identified ROS, RNS and the loss of the antioxidant defense, not only as effects but also as contributors to asthma pathogenesis [182-184]. For instance, bronchoalveolar lavage fluid (BALF) of asthmatics exhibited $50 \%$ reduction in the activity of the antioxidant enzyme catalase compared to healthy controls. This reduction in activity was accompanied by oxidation of catalase at cys 377 [185], which likely amplified oxidative stress and contributed to the inflammatory state of the asthmatic airway. In fact, higher levels of nitric oxide, eosinophil-mediated oxidative tissue injury and bioactive lipid oxidation products are some of the characteristic features of asthma [186-189]. Elevated levels of eosinophil peroxidase and myeloperoxidase were found to parallel numbers of eosinophils and neutrophils, respectively, and were increased in asthmatic peripheral blood, sputum and BALF [184, 190, 191]. Importantly, asthma and asthma exacerbations elicit changes in intracellular and extracellular GSH and GSSG, leading to rapid changes in the redox potential immediately following antigen challenge in epithelial lining fluid of asthmatics [192]. This altered redox signaling induces protein S-glutathionylation that can be reversed by glutaredoxins and thioredoxins [193]. Interestingly, Glutaredoxin 1 (GLRX1) was found to be increased in a murine mouse model of asthma [194], as well as in sputum derived from asthmatics [195]. The nuclear factor-kB (NF-kB), that regulates multiple inflammatory genes responsible for asthma pathogenesis, is another example of targets modulated by S-glutathionylation and S-nitrosylation in lung epithelial cells [196, 197]. Additionally, the glutathione S-transferase (GST) family, including GSTP1 and GSTM, has also been implicated in asthma pathophysiology [198]. Together, these findings confirm the implications of oxidative stress and altered redox balance in 
asthma pathogenesis. Moreover, activation of mast cells, dendritic cells, neutrophils as well as polarization of the proinflammatory macrophages are all accompanied by increases in glycolysis and shifts towards the PPP and fatty acid synthesis [199-202]. This metabolic shift provides the macromolecules required for proper functioning of the immune cells. On the other hand, the antiinflammatory macrophages utilize the fatty acid oxidation and oxidative phosphorylation to generate sufficient energy required for prolonging survival [178, 202]. Other studies highlighted the role played by TLR-mediated increase in glycolysis and its necessity for the proper activation and function of DCs [200, 203]. Inhibition of type 2 innate lymphoid cells (ILC2)-driven IL33 by PKM2 is yet another example of how glycolysis and metabolic reprogramming affect asthma progression [204]. In addition, modulation of the glycolysis pathway via phosphorylation and inactivation of PKM2 had been shown to be indispensable for mast cells degranulation [205]. Interestingly, airway epithelial cells have recently been shown to be modulated by glycolytic reprogramming and redox homeostasis. For instance, airway epithelial cells derived from asthmatics exhibit increases in mitochondrial numbers and enhanced oxidative phosphorylation due to the arginine metabolism reprogramming [206]. In addition, increased glycolysis was demonstrated in epithelial cells and platelets of obese asthmatics compared to lean asthmatics and healthy subjects, pointing at a potential role of metabolic reprogramming in epithelial cells in asthma pathogenesis [207]. It is obvious then that metabolic reprogramming and redox balance may contribute to asthma pathogenesis, however, there is still a lot to be unraveled. 


\section{REFERENCES:}

1. DeBerardinis, R.J. and C.B. Thompson, Cellular metabolism and disease: what do metabolic outliers teach us? Cell, 2012. 148(6): p. 1132-44.

2. Lévy, P. and B. Bartosch, Metabolic reprogramming: a hallmark of viral oncogenesis. Oncogene, 2016. 35(32): p. 4155-64.

3. Warburg, O., On the origin of cancer cells. Science, 1956. 123(3191): p. 309-14.

4. Boroughs, L.K. and R.J. DeBerardinis, Metabolic pathways promoting cancer cell survival and growth. Nat Cell Biol, 2015. 17(4): p. 351-9.

5. Deberardinis, R.J., et al., Brick by brick: metabolism and tumor cell growth. Curr Opin Genet Dev, 2008. 18(1): p. 54-61.

6. Yan, X., et al., Metabolic Dysregulation Contributes to the Progression of Alzheimer's Disease. Front Neurosci, 2020. 14: p. 530219.

7. Veijola, R., et al., Dysregulation of glucose metabolism in preclinical type 1 diabetes. Pediatr Diabetes, 2016. 17 Suppl 22: p. 25-30.

8. Chen, H., et al., Lipid metabolism in chronic obstructive pulmonary disease. Int J Chron Obstruct Pulmon Dis, 2019. 14: p. 1009-1018.

9. Rastogi, D., et al., Inflammation, metabolic dysregulation, and pulmonary function among obese urban adolescents with asthma. Am J Respir Crit Care Med, 2015. 191(2): p. 149-60.

10. Pite, H., et al., Metabolic Dysfunction and Asthma: Current Perspectives. J Asthma Allergy, 2020. 13: p. 237-247.

11. Park, S., et al., The relationship between metabolic syndrome and asthma in the elderly. Sci Rep, 2018. 8(1): p. 9378.

12. Abdel-Haleem, A.M., et al., The Emerging Facets of Non-Cancerous Warburg Effect. Front Endocrinol (Lausanne), 2017. 8: p. 279.

13. Palsson-McDermott, E.M. and L.A. O'Neill, The Warburg effect then and now: from cancer to inflammatory diseases. Bioessays, 2013. 35(11): p. 965-73.

14. Johnson, R.J., et al., Hypothesis: could excessive fructose intake and uric acid cause type 2 diabetes? Endocr Rev, 2009. 30(1): p. 96-116.

15. Kosow, D.P. and I.A. Rose, Product inhibition of the hexokinases. J Biol Chem, 1970. 245(1): p. 198204.

16. Šmerc, A., E. Sodja, and M. Legiša, Posttranslational modification of 6-phosphofructo-1-kinase as an important feature of cancer metabolism. PLoS One, 2011. 6(5): p. e19645.

17. Prakasam, G., et al., Posttranslational Modifications of Pyruvate Kinase M2: Tweaks that Benefit Cancer. Front Oncol, 2018. 8: p. 22.

18. Wilson, J.E., Hexokinases. Rev Physiol Biochem Pharmacol, 1995. 126: p. 65-198.

19. Board, M., A. Colquhoun, and E.A. Newsholme, High Km glucose-phosphorylating (glucokinase) activities in a range of tumor cell lines and inhibition of rates of tumor growth by the specific enzyme inhibitor mannoheptulose. Cancer Res, 1995. 55(15): p. 3278-85.

20. Roberts, D.J. and S. Miyamoto, Hexokinase II integrates energy metabolism and cellular protection: Akting on mitochondria and TORCing to autophagy. Cell Death Differ, 2015. 22(2): p. 248-57.

21. Wilson, J.E., Isozymes of mammalian hexokinase: structure, subcellular localization and metabolic function. J Exp Biol, 2003. 206(Pt 12): p. 2049-57.

22. Uyeda, K., Phosphofructokinase. Adv Enzymol Relat Areas Mol Biol, 1979. 48: p. 193-244.

23. Mansour, T.E., Phosphofructokinase. Curr Top Cell Regul, 1972. 5: p. 1-46.

24. Dobson, G.P., E. Yamamoto, and P.W. Hochachka, Phosphofructokinase control in muscle: nature and reversal of pH-dependent ATP inhibition. Am J Physiol, 1986. 250(1 Pt 2): p. R71-6. 
25. Jenkins, C.M., et al., Reversible high affinity inhibition of phosphofructokinase-1 by acyl-CoA: a mechanism integrating glycolytic flux with lipid metabolism. J Biol Chem, 2011. 286(14): p. 1193750.

26. Kemp, R.G. and L.G. Foe, Allosteric regulatory properties of muscle phosphofructokinase. Mol Cell Biochem, 1983. 57(2): p. 147-54.

27. Uyeda, K., E. Furuya, and L.J. Luby, The effect of natural and synthetic D-fructose 2,6-bisphosphate on the regulatory kinetic properties of liver and muscle phosphofructokinases. J Biol Chem, 1981. 256(16): p. 8394-9.

28. Webb, B.A., et al., Structures of human phosphofructokinase-1 and atomic basis of cancerassociated mutations. Nature, 2015. 523(7558): p. 111-4.

29. Mazurek, S., Pyruvate kinase type M2: a key regulator of the metabolic budget system in tumor cells. Int J Biochem Cell Biol, 2011. 43(7): p. 969-80.

30. Netzker, R., et al., Cell cycle-associated expression of M2-type isozyme of pyruvate kinase in proliferating rat thymocytes. J Biol Chem, 1992. 267(9): p. 6421-4.

31. Israelsen, W.J. and M.G. Vander Heiden, Pyruvate kinase: Function, regulation and role in cancer. Semin Cell Dev Biol, 2015. 43: p. 43-51.

32. Kung, C., et al., AG-348 enhances pyruvate kinase activity in red blood cells from patients with pyruvate kinase deficiency. Blood, 2017. 130(11): p. 1347-1356.

33. Dombrauckas, J.D., B.D. Santarsiero, and A.D. Mesecar, Structural basis for tumor pyruvate kinase M2 allosteric regulation and catalysis. Biochemistry, 2005. 44(27): p. 9417-29.

34. Macpherson, J.A., et al., Functional cross-talk between allosteric effects of activating and inhibiting ligands underlies PKM2 regulation. Elife, 2019. 8.

35. Chaneton, B., et al., Serine is a natural ligand and allosteric activator of pyruvate kinase M2. Nature, 2012. 491(7424): p. 458-462.

36. de Koning, T.J., et al., L-serine in disease and development. Biochem J, 2003. 371(Pt 3): p. 653-61.

37. Carbonell, J., et al., Pyruvate kinase. Classes of regulatory isoenzymes in mammalian tissues. Eur J Biochem, 1973. 37(1): p. 148-56.

38. Yang, W. and Z. Lu, Regulation and function of pyruvate kinase M2 in cancer. Cancer Lett, 2013. 339(2): p. 153-8.

39. Davis, P.J. and F.B. Davis, Nongenomic actions of thyroid hormone. Thyroid, 1996. 6(5): p. 497504.

40. Hitosugi, T., et al., Tyrosine phosphorylation inhibits PKM2 to promote the Warburg effect and tumor growth. Sci Signal, 2009. 2(97): p. ra73.

41. Anastasiou, D., et al., Inhibition of pyruvate kinase $M 2$ by reactive oxygen species contributes to cellular antioxidant responses. Science, 2011. 334(6060): p. 1278-83.

42. Lv, L., et al., Acetylation targets the M2 isoform of pyruvate kinase for degradation through chaperone-mediated autophagy and promotes tumor growth. Mol Cell, 2011. 42(6): p. 719-30.

43. Liu, F., et al., PKM2 methylation by CARM1 activates aerobic glycolysis to promote tumorigenesis. Nat Cell Biol, 2017. 19(11): p. 1358-1370.

44. Liu, K., et al., Parkin Regulates the Activity of Pyruvate Kinase M2. J Biol Chem, 2016. 291(19): p. 10307-17.

45. Luo, W., et al., Pyruvate kinase M2 is a PHD3-stimulated coactivator for hypoxia-inducible factor 1. Cell, 2011. 145(5): p. 732-44.

46. Wang, Y., et al., O-GICNAcylation destabilizes the active tetrameric PKM2 to promote the Warburg effect. Proc Natl Acad Sci U S A, 2017. 114(52): p. 13732-13737.

47. Spoden, G.A., et al., The SUMO-E3 ligase PIAS3 targets pyruvate kinase M2. J Cell Biochem, 2009. 107(2): p. 293-302. 
48. Patra, K.C. and N. Hay, The pentose phosphate pathway and cancer. Trends Biochem Sci, 2014. 39(8): p. 347-54.

49. Bohme, C.C., et al., Kinetic characterization of glutathione reductase from the malarial parasite Plasmodium falciparum. Comparison with the human enzyme. J Biol Chem, 2000. 275(48): p. 37317-23.

50. Tarafdar, A. and G. Pula, The Role of NADPH Oxidases and Oxidative Stress in Neurodegenerative Disorders. Int J Mol Sci, 2018. 19(12).

51. Dunnill, C., et al., Reactive oxygen species (ROS) and wound healing: the functional role of ROS and emerging ROS-modulating technologies for augmentation of the healing process. Int Wound J, 2017. 14(1): p. 89-96.

52. Puertollano, M.A., et al., Dietary antioxidants: immunity and host defense. Curr Top Med Chem, 2011. 11(14): p. 1752-66.

53. Scherz-Shouval, R., et al., Reactive oxygen species are essential for autophagy and specifically regulate the activity of Atg4. Embo j, 2007. 26(7): p. 1749-60.

54. Zhang, X., et al., MCOLN1 is a ROS sensor in lysosomes that regulates autophagy. Nat Commun, 2016. 7: p. 12109.

55. Izeradjene, K., et al., Reactive oxygen species regulate caspase activation in tumor necrosis factorrelated apoptosis-inducing ligand-resistant human colon carcinoma cell lines. Cancer Res, 2005. 65(16): p. 7436-45.

56. Kamata, H., et al., Reactive oxygen species promote TNFalpha-induced death and sustained JNK activation by inhibiting MAP kinase phosphatases. Cell, 2005. 120(5): p. 649-61.

57. Cho, Y.S., et al., Phosphorylation-driven assembly of the RIP1-RIP3 complex regulates programmed necrosis and virus-induced inflammation. Cell, 2009. 137(6): p. 1112-23.

58. Jones, R.M., et al., Symbiotic lactobacilli stimulate gut epithelial proliferation via Nox-mediated generation of reactive oxygen species. Embo j, 2013. 32(23): p. 3017-28.

59. Janssen-Heininger, Y.M., et al., Redox-based regulation of signal transduction: principles, pitfalls, and promises. Free Radic Biol Med, 2008. 45(1): p. 1-17.

60. Akerboom, T.P., M. Bilzer, and H. Sies, The relationship of biliary glutathione disulfide efflux and intracellular glutathione disulfide content in perfused rat liver. J Biol Chem, 1982. 257(8): p. 424852.

61. Seelig, G.F., R.P. Simondsen, and A. Meister, Reversible dissociation of gamma-glutamylcysteine synthetase into two subunits. J Biol Chem, 1984. 259(15): p. 9345-7.

62. Huang, C.S., et al., Catalytic and regulatory properties of the heavy subunit of rat kidney gammaglutamylcysteine synthetase. J Biol Chem, 1993. 268(26): p. 19675-80.

63. Liu, Y., et al., Emerging regulatory paradigms in glutathione metabolism. Adv Cancer Res, 2014. 122: p. 69-101.

64. Lu, S.C., Glutathione synthesis. Biochim Biophys Acta, 2013. 1830(5): p. 3143-53.

65. Chia, S.B., et al., Dysregulation of the glutaredoxin/S-glutathionylation redox axis in lung diseases. Am J Physiol Cell Physiol, 2020. 318(2): p. C304-c327.

66. Kumar, A., et al., Mammalian proapoptotic factor ChaC1 and its homologues function as $\gamma$ glutamyl cyclotransferases acting specifically on glutathione. EMBO Rep, 2012. 13(12): p. 1095101.

67. Kaur, A., et al., ChaC2, an Enzyme for Slow Turnover of Cytosolic Glutathione. J Biol Chem, 2017. 292(2): p. 638-651.

68. Mungrue, I.N., et al., CHAC1/MGC4504 is a novel proapoptotic component of the unfolded protein response, downstream of the ATF4-ATF3-CHOP cascade. J Immunol, 2009. 182(1): p. 466-76.

69. Joo, N.E., et al., Nisin, an apoptogenic bacteriocin and food preservative, attenuates HNSCC tumorigenesis via CHAC1. Cancer Med, 2012. 1(3): p. 295-305. 
70. Goebel, G., et al., Elevated mRNA expression of CHAC1 splicing variants is associated with poor outcome for breast and ovarian cancer patients. Br J Cancer, 2012. 106(1): p. 189-98.

71. Boyland, E. and L.F. Chasseaud, The role of glutathione and glutathione S-transferases in mercapturic acid biosynthesis. Adv Enzymol Relat Areas Mol Biol, 1969. 32: p. 173-219.

72. Huang, Z.Z., et al., Mechanism and significance of increased glutathione level in human hepatocellular carcinoma and liver regeneration. Faseb j, 2001. 15(1): p. 19-21.

73. Carretero, J., et al., Growth-associated changes in glutathione content correlate with liver metastatic activity of B16 melanoma cells. Clin Exp Metastasis, 1999. 17(7): p. 567-74.

74. Locksley, R.M., Asthma and allergic inflammation. Cell, 2010. 140(6): p. 777-83.

75. Mayatepek, E., et al., Deficient synthesis of cysteinyl leukotrienes in glutathione synthetase deficiency. Int J Tissue React, 1993. 15(6): p. 245-52.

76. Rogan, E.G., et al., Relative imbalances in estrogen metabolism and conjugation in breast tissue of women with carcinoma: potential biomarkers of susceptibility to cancer. Carcinogenesis, 2003. 24(4): p. 697-702.

77. Margalit, A., et al., Regulation of prostaglandin biosynthesis in vivo by glutathione. Am J Physiol, 1998. 274(2): p. R294-302.

78. Suthanthiran, M., et al., Glutathione regulates activation-dependent DNA synthesis in highly purified normal human T lymphocytes stimulated via the CD2 and CD3 antigens. Proc Natl Acad Sci U S A, 1990. 87(9): p. 3343-7.

79. Dickinson, D.A. and H.J. Forman, Cellular glutathione and thiols metabolism. Biochem Pharmacol, 2002. 64(5-6): p. 1019-26.

80. Sakai, J., et al., Reactive oxygen species-induced actin glutathionylation controls actin dynamics in neutrophils. Immunity, 2012. 37(6): p. 1037-49.

81. Wang, J., et al., Reversible glutathionylation regulates actin polymerization in A431 cells. J Biol Chem, 2001. 276(51): p. 47763-6.

82. van der Linde, K., et al., Regulation of plant cytosolic aldolase functions by redox-modifications. Plant Physiol Biochem, 2011. 49(9): p. 946-57.

83. Mailloux, R.J., et al., Glutathionylation acts as a control switch for uncoupling proteins UCP2 and UCP3. J Biol Chem, 2011. 286(24): p. 21865-75.

84. Passarelli, C., et al., GSSG-mediated Complex I defect in isolated cardiac mitochondria. Int J Mol Med, 2010. 26(1): p. 95-9.

85. Kambe, T., et al., Inactivation of $\mathrm{Ca}+\mathrm{+} /$ calmodulin-dependent protein kinase I by $S$ glutathionylation of the active-site cysteine residue. FEBS Lett, 2010. 584(11): p. 2478-84.

86. Silva, G.M., et al., Redox control of 205 proteasome gating. Antioxid Redox Signal, 2012. 16(11): p. 1183-94.

87. Dong, K., et al., Glutaredoxins concomitant with optimal ROS activate AMPK through Sglutathionylation to improve glucose metabolism in type 2 diabetes. Free Radic Biol Med, 2016. 101: p. 334-347.

88. Gorelenkova Miller, O., et al., Upregulation of Glutaredoxin-1 Activates Microglia and Promotes Neurodegeneration: Implications for Parkinson's Disease. Antioxid Redox Signal, 2016. 25(18): p. 967-982.

89. Weinberg, E.O., et al., IL-33 induction and signaling are controlled by glutaredoxin-1 in mouse macrophages. PLoS One, 2019. 14(1): p. e0210827.

90. Anathy, V., et al., Reducing protein oxidation reverses lung fibrosis. Nat Med, 2018. 24(8): p. 11281135.

91. Tian, L., M.M. Shi, and H.J. Forman, Increased transcription of the regulatory subunit of gammaglutamylcysteine synthetase in rat lung epithelial L2 cells exposed to oxidative stress or glutathione depletion. Arch Biochem Biophys, 1997. 342(1): p. 126-33. 
92. Rahman, I., et al., Induction of gamma-glutamylcysteine synthetase by cigarette smoke is associated with AP-1 in human alveolar epithelial cells. FEBS Lett, 1996. 396(1): p. 21-5.

93. Kensler, T.W., N. Wakabayashi, and S. Biswal, Cell survival responses to environmental stresses via the Keap1-Nrf2-ARE pathway. Annu Rev Pharmacol Toxicol, 2007. 47: p. 89-116.

94. Jyrkkänen, H.K., et al., Nrf2 regulates antioxidant gene expression evoked by oxidized phospholipids in endothelial cells and murine arteries in vivo. Circ Res, 2008. 103(1): p. e1-9.

95. Peng, Z., et al., Inhibitor of kappaB kinase beta regulates redox homeostasis by controlling the constitutive levels of glutathione. Mol Pharmacol, 2010. 77(5): p. 784-92.

96. Benassi, B., et al., c-Myc phosphorylation is required for cellular response to oxidative stress. Mol Cell, 2006. 21(4): p. 509-19.

97. Zhu, W., et al., The anthocyanin cyanidin-3-O-B-glucoside, a flavonoid, increases hepatic glutathione synthesis and protects hepatocytes against reactive oxygen species during hyperglycemia: Involvement of a CAMP-PKA-dependent signaling pathway. Free Radic Biol Med, 2012. 52(2): p. 314-27.

98. Fu, Y., et al., Epigallocatechin-3-gallate inhibits growth of activated hepatic stellate cells by enhancing the capacity of glutathione synthesis. Mol Pharmacol, 2008. 73(5): p. 1465-73.

99. Jardine, H., et al., Molecular mechanism of transforming growth factor (TGF)-beta1-induced glutathione depletion in alveolar epithelial cells. Involvement of AP-1/ARE and Fra-1. J Biol Chem, 2002. 277(24): p. 21158-66.

100. Sun, W.M., Z.Z. Huang, and S.C. Lu, Regulation of gamma-glutamylcysteine synthetase by protein phosphorylation. Biochem J, 1996. 320 ( Pt 1)(Pt 1): p. 321-8.

101. Franklin, C.C., et al., TGFbeta1-induced suppression of glutathione antioxidant defenses in hepatocytes: caspase-dependent post-translational and caspase-independent transcriptional regulatory mechanisms. Faseb j, 2003. 17(11): p. 1535-7.

102. Lee, T.D., et al., Cloning and characterization of the human glutathione synthetase 5'-flanking region. Biochem J, 2005. 390(Pt 2): p. 521-8.

103. Yang, H., et al., Tumour necrosis factor alpha induces co-ordinated activation of rat GSH synthetic enzymes via nuclear factor kappaB and activator protein-1. Biochem J, 2005. 391(Pt 2): p. 399408.

104. Lu, S.C., Regulation of glutathione synthesis. Mol Aspects Med, 2009. 30(1-2): p. 42-59.

105. Lu, S.C., Regulation of hepatic glutathione synthesis: current concepts and controversies. Faseb j, 1999. 13(10): p. 1169-83.

106. Bannai, S. and N. Tateishi, Role of membrane transport in metabolism and function of glutathione in mammals. J Membr Biol, 1986. 89(1): p. 1-8.

107. Bannai, S. and E. Kitamura, Transport interaction of L-cystine and L-glutamate in human diploid fibroblasts in culture. J Biol Chem, 1980. 255(6): p. 2372-6.

108. Verrey, F., et al., CATs and HATs: the SLC7 family of amino acid transporters. Pflugers Arch, 2004. 447(5): p. 532-42.

109. Sato, H., et al., Cloning and expression of a plasma membrane cystine/glutamate exchange transporter composed of two distinct proteins. J Biol Chem, 1999. 274(17): p. 11455-8.

110. Lewerenz, J., et al., The cystine/glutamate antiporter system x(c)(-) in health and disease: from molecular mechanisms to novel therapeutic opportunities. Antioxid Redox Signal, 2013. 18(5): p. 522-55.

111. Mohan, A., et al., Molecular diffusion model of neurotransmitter homeostasis around synapses supporting gradients. Neural Comput, 2011. 23(4): p. 984-1014.

112. Kalivas, P.W., et al., Glutamate transmission in addiction. Neuropharmacology, 2009. 56 Suppl 1(Suppl 1): p. 169-73. 
113. Baker, D.A., et al., Neuroadaptations in cystine-glutamate exchange underlie cocaine relapse. Nat Neurosci, 2003. 6(7): p. 743-9.

114. Amen, S.L., et al., Repeated N-acetyl cysteine reduces cocaine seeking in rodents and craving in cocaine-dependent humans. Neuropsychopharmacology, 2011. 36(4): p. 871-8.

115. Knackstedt, L.A., et al., The role of cystine-glutamate exchange in nicotine dependence in rats and humans. Biol Psychiatry, 2009. 65(10): p. 841-5.

116. Hu, R.G., et al., Characterization of the cystine/glutamate transporter in the outer plexiform layer of the vertebrate retina. Eur J Neurosci, 2008. 28(8): p. 1491-502.

117. Barger, S.W. and A.S. Basile, Activation of microglia by secreted amyloid precursor protein evokes release of glutamate by cystine exchange and attenuates synaptic function. J Neurochem, 2001. 76(3): p. 846-54.

118. Massie, A., et al., Time-dependent changes in striatal xCT protein expression in hemi-Parkinson rats. Neuroreport, 2008. 19(16): p. 1589-92.

119. Domercq, M., et al., System xc- and glutamate transporter inhibition mediates microglial toxicity to oligodendrocytes. J Immunol, 2007. 178(10): p. 6549-56.

120. Taguchi, K., et al., Induction of cystine/glutamate transporter in bacterial lipopolysaccharide induced endotoxemia in mice. J Inflamm (Lond), 2007. 4: p. 20.

121. Lin, W., et al., SLC7A11/XCT in cancer: biological functions and therapeutic implications. Am J Cancer Res, 2020. 10(10): p. 3106-3126.

122. de Groot, J. and H. Sontheimer, Glutamate and the biology of gliomas. Glia, 2011. 59(8): p. 11819.

123. Ishii, T., et al., Transcription factor Nrf2 coordinately regulates a group of oxidative stress-inducible genes in macrophages. J Biol Chem, 2000. 275(21): p. 16023-9.

124. Bannai, S. and E. Kitamura, Adaptive enhancement of cystine and glutamate uptake in human diploid fibroblasts in culture. Biochim Biophys Acta, 1982. 721(1): p. 1-10.

125. Sato, H., et al., Transcriptional control of cystine/glutamate transporter gene by amino acid deprivation. Biochem Biophys Res Commun, 2004. 325(1): p. 109-16.

126. Koppula, P., et al., The glutamate/cystine antiporter SLC7A11/xCT enhances cancer cell dependency on glucose by exporting glutamate. J Biol Chem, 2017. 292(34): p. 14240-14249.

127. Gupta, S., A decision between life and death during TNF-alpha-induced signaling. J Clin Immunol, 2002. 22(4): p. 185-94.

128. Sato, H., et al., Induction of cystine transport activity in mouse peritoneal macrophages by bacterial lipopolysaccharide. Biochem J, 1995. 310 ( Pt 2)(Pt 2): p. 547-51.

129. Limón-Pacheco, J.H., et al., Glutathione depletion activates mitogen-activated protein kinase (MAPK) pathways that display organ-specific responses and brain protection in mice. Free Radic Biol Med, 2007. 43(9): p. 1335-47.

130. Sasaki, H., et al., Electrophile response element-mediated induction of the cystine/glutamate exchange transporter gene expression. J Biol Chem, 2002. 277(47): p. 44765-71.

131. Giudice, A., C. Arra, and M.C. Turco, Review of molecular mechanisms involved in the activation of the Nrf2-ARE signaling pathway by chemopreventive agents. Methods Mol Biol, 2010. 647: p. 3774.

132. Lewerenz, J., et al., Induction of Nrf2 and $x C T$ are involved in the action of the neuroprotective antibiotic ceftriaxone in vitro. J Neurochem, 2009. 111(2): p. 332-43.

133. Ishimoto, T., et al., CD44 variant regulates redox status in cancer cells by stabilizing the xCT subunit of system xc(-) and thereby promotes tumor growth. Cancer Cell, 2011. 19(3): p. 387-400.

134. Lewerenz, J., M. Klein, and A. Methner, Cooperative action of glutamate transporters and cystine/glutamate antiporter system Xc- protects from oxidative glutamate toxicity. J Neurochem, 2006. 98(3): p. 916-25. 
135. Patel, S.A., et al., Differentiation of substrate and non-substrate inhibitors of transport system xc(): an obligate exchanger of L-glutamate and L-cystine. Neuropharmacology, 2004. 46(2): p. 27384.

136. Bannai, S. and T. Ishii, A novel function of glutamine in cell culture: utilization of glutamine for the uptake of cystine in human fibroblasts. J Cell Physiol, 1988. 137(2): p. 360-6.

137. Gu, Y., et al., mTORC2 Regulates Amino Acid Metabolism in Cancer by Phosphorylation of the Cystine-Glutamate Antiporter xCT. Mol Cell, 2017. 67(1): p. 128-138.e7.

138. Liu, T., et al., The Deubiquitylase OTUB1 Mediates Ferroptosis via Stabilization of SLC7A11. Cancer Res, 2019. 79(8): p. 1913-1924.

139. Bargagli, E., et al., Oxidative stress in the pathogenesis of diffuse lung diseases: a review. Respir Med, 2009. 103(9): p. 1245-56.

140. Rogers, L.K. and M.J. Cismowski, Oxidative Stress in the Lung - The Essential Paradox. Curr Opin Toxicol, 2018. 7: p. 37-43.

141. Auten, R.L. and J.M. Davis, Oxygen toxicity and reactive oxygen species: the devil is in the details. Pediatr Res, 2009. 66(2): p. 121-7.

142. Kirkham, P.A. and P.J. Barnes, Oxidative stress in COPD. Chest, 2013. 144(1): p. 266-273.

143. Cantin, A.M., et al., Normal alveolar epithelial lining fluid contains high levels of glutathione. J Appl Physiol (1985), 1987. 63(1): p. 152-7.

144. Mårtensson, J., et al., Glutathione metabolism in the lung: inhibition of its synthesis leads to lamellar body and mitochondrial defects. Proc Natl Acad Sci U S A, 1989. 86(14): p. 5296-300.

145. John, J., et al., Regional Disparity in Asthma Prevalence and Distribution of Asthma Education Programs in Texas. J Environ Public Health, 2020. 2020: p. 9498124.

146. Dharmage, S.C., J.L. Perret, and A. Custovic, Epidemiology of Asthma in Children and Adults. Front Pediatr, 2019. 7: p. 246.

147. Standards for the diagnosis and care of patients with chronic obstructive pulmonary disease (COPD) and asthma. This official statement of the American Thoracic Society was adopted by the ATS Board of Directors, November 1986. Am Rev Respir Dis, 1987. 136(1): p. 225-44.

148. Liu, M.C., et al., Immediate and late inflammatory responses to ragweed antigen challenge of the peripheral airways in allergic asthmatics. Cellular, mediator, and permeability changes. Am Rev Respir Dis, 1991. 144(1): p. 51-8.

149. Bradding, P. and G. Arthur, Mast cells in asthma--state of the art. Clin Exp Allergy, 2016. 46(2): $\mathrm{p}$. 194-263.

150. Zhu, Z., et al., Pulmonary expression of interleukin-13 causes inflammation, mucus hypersecretion, subepithelial fibrosis, physiologic abnormalities, and eotaxin production. J Clin Invest, 1999. 103(6): p. 779-88.

151. Lambrecht, B.N., H. Hammad, and J.V. Fahy, The Cytokines of Asthma. Immunity, 2019. 50(4): p. 975-991.

152. Simons, F.E., Anaphylaxis. J Allergy Clin Immunol, 2010. 125(2 Suppl 2): p. S161-81.

153. McWilliam, A.S., et al., Rapid dendritic cell recruitment is a hallmark of the acute inflammatory response at mucosal surfaces. J Exp Med, 1994. 179(4): p. 1331-6.

154. Hammad, H., et al., Inflammatory dendritic cells--not basophils--are necessary and sufficient for induction of Th2 immunity to inhaled house dust mite allergen. J Exp Med, 2010. 207(10): p. 2097111.

155. Holgate, S.T., Innate and adaptive immune responses in asthma. Nat Med, 2012. 18(5): p. 673-83. 156. Holgate, S.T., et al., Asthma. Nat Rev Dis Primers, 2015. 1(1): p. 15025.

157. Hammad, H. and B.N. Lambrecht, Dendritic cells and epithelial cells: linking innate and adaptive immunity in asthma. Nat Rev Immunol, 2008. 8(3): p. 193-204. 
158. Cates, E.C., et al., Intranasal exposure of mice to house dust mite elicits allergic airway inflammation via a GM-CSF-mediated mechanism. J Immunol, 2004. 173(10): p. 6384-92.

159. Hammad, H., et al., House dust mite allergen induces asthma via Toll-like receptor 4 triggering of airway structural cells. Nat Med, 2009. 15(4): p. 410-6.

160. Nagarkar, D.R., et al., Airway epithelial cells activate TH2 cytokine production in mast cells through IL-1 and thymic stromal lymphopoietin. J Allergy Clin Immunol, 2012. 130(1): p. 225-32.e4.

161. Moore, W.C., et al., Identification of asthma phenotypes using cluster analysis in the Severe Asthma Research Program. Am J Respir Crit Care Med, 2010. 181(4): p. 315-23.

162. Shaw, D.E., et al., Clinical and inflammatory characteristics of the European U-BIOPRED adult severe asthma cohort. Eur Respir J, 2015. 46(5): p. 1308-21.

163. Loza, M.J., et al., Validated and longitudinally stable asthma phenotypes based on cluster analysis of the ADEPT study. Respir Res, 2016. 17(1): p. 165.

164. Dougherty, R.H., et al., Accumulation of intraepithelial mast cells with a unique protease phenotype in T(H)2-high asthma. J Allergy Clin Immunol, 2010. 125(5): p. 1046-1053.e8.

165. Kuruvilla, M.E., F.E. Lee, and G.B. Lee, Understanding Asthma Phenotypes, Endotypes, and Mechanisms of Disease. Clin Rev Allergy Immunol, 2019. 56(2): p. 219-233.

166. McGrath, K.W., et al., A large subgroup of mild-to-moderate asthma is persistently noneosinophilic. Am J Respir Crit Care Med, 2012. 185(6): p. 612-9.

167. Carr, T.F. and M. Kraft, Chronic Infection and Severe Asthma. Immunol Allergy Clin North Am, 2016. 36(3): p. 483-502.

168. Fahy, J.V., Type 2 inflammation in asthma--present in most, absent in many. Nat Rev Immunol, 2015. 15(1): p. 57-65.

169. Juniper, E.F., et al., Effect of long-term treatment with an inhaled corticosteroid (budesonide) on airway hyperresponsiveness and clinical asthma in nonsteroid-dependent asthmatics. Am Rev Respir Dis, 1990. 142(4): p. 832-6.

170. Juniper, E.F., et al., Long-term effects of budesonide on airway responsiveness and clinical asthma severity in inhaled steroid-dependent asthmatics. Eur Respir J, 1990. 3(10): p. 1122-7.

171. Dahl, R., et al., A dose-ranging study of fluticasone propionate in adult patients with moderate asthma. International Study Group. Chest, 1993. 104(5): p. 1352-8.

172. Pauwels, R.A., et al., Effect of inhaled formoterol and budesonide on exacerbations of asthma. Formoterol and Corticosteroids Establishing Therapy (FACET) International Study Group. N Engl J Med, 1997. 337(20): p. 1405-11.

173. O'Byrne, P.M., et al., Budesonide/formoterol combination therapy as both maintenance and reliever medication in asthma. Am J Respir Crit Care Med, 2005. 171(2): p. 129-36.

174. Reiss, T.F., et al., Montelukast, a once-daily leukotriene receptor antagonist, in the treatment of chronic asthma: a multicenter, randomized, double-blind trial. Montelukast Clinical Research Study Group. Arch Intern Med, 1998. 158(11): p. 1213-20.

175. Busse, W., et al., Omalizumab, anti-IgE recombinant humanized monoclonal antibody, for the treatment of severe allergic asthma. J Allergy Clin Immunol, 2001. 108(2): p. 184-90.

176. Chen, M., et al., Overlap of allergic, eosinophilic and type 2 inflammatory subtypes in moderateto-severe asthma. Clin Exp Allergy, 2021. 51(4): p. 546-555.

177. Hiemstra, P.S., P.B. McCray, Jr., and R. Bals, The innate immune function of airway epithelial cells in inflammatory lung disease. Eur Respir J, 2015. 45(4): p. 1150-62.

178. Van den Bossche, J., L.A. O'Neill, and D. Menon, Macrophage Immunometabolism: Where Are We (Going)? Trends Immunol, 2017. 38(6): p. 395-406.

179. Dweik, R.A., et al., NO chemical events in the human airway during the immediate and late antigen-induced asthmatic response. Proc Natl Acad Sci U S A, 2001. 98(5): p. 2622-7. 
180. Gaston, B., et al., The biology of nitrogen oxides in the airways. Am J Respir Crit Care Med, 1994. 149(2 Pt 1): p. 538-51.

181. MacPherson, J.C., et al., Eosinophils are a major source of nitric oxide-derived oxidants in severe asthma: characterization of pathways available to eosinophils for generating reactive nitrogen species. J Immunol, 2001. 166(9): p. 5763-72.

182. Mabalirajan, U., et al., Mitochondrial structural changes and dysfunction are associated with experimental allergic asthma. J Immunol, 2008. 181(5): p. 3540-8.

183. Aguilera-Aguirre, L., et al., Mitochondrial dysfunction increases allergic airway inflammation. J Immunol, 2009. 183(8): p. 5379-87.

184. Comhair, S.A. and S.C. Erzurum, Redox control of asthma: molecular mechanisms and therapeutic opportunities. Antioxid Redox Signal, 2010. 12(1): p. 93-124.

185. Ghosh, S., et al., Nitrotyrosine proteome survey in asthma identifies oxidative mechanism of catalase inactivation. J Immunol, 2006. 176(9): p. 5587-97.

186. Guo, F.H., et al., Molecular mechanisms of increased nitric oxide (NO) in asthma: evidence for transcriptional and post-translational regulation of NO synthesis. J Immunol, 2000. 164(11): p. 5970-80.

187. Kharitonov, S.A., et al., Increased nitric oxide in exhaled air of asthmatic patients. Lancet, 1994. 343(8890): p. 133-5.

188. Montuschi, P., et al., Increased 8-isoprostane, a marker of oxidative stress, in exhaled condensate of asthma patients. Am J Respir Crit Care Med, 1999. 160(1): p. 216-20.

189. Piotrowski, W.J., et al., Exhaled breath 8-isoprostane as a marker of asthma severity. Arch Med Sci, 2012. 8(3): p. 515-20.

190. Bolscher, B.G., H. Plat, and R. Wever, Some properties of human eosinophil peroxidase, a comparison with other peroxidases. Biochim Biophys Acta, 1984. 784(2-3): p. 177-86.

191. Olsen, R.L. and C. Little, Purification and some properties of myeloperoxidase and eosinophil peroxidase from human blood. Biochem J, 1983. 209(3): p. 781-7.

192. Comhair, S.A., et al., Rapid loss of superoxide dismutase activity during antigen-induced asthmatic response. Lancet, 2000. 355(9204): p. 624.

193. Dalle-Donne, I., et al., Molecular mechanisms and potential clinical significance of Sglutathionylation. Antioxid Redox Signal, 2008. 10(3): p. 445-73.

194. Reynaert, N.L., E.F. Wouters, and Y.M. Janssen-Heininger, Modulation of glutaredoxin-1 expression in a mouse model of allergic airway disease. Am J Respir Cell Mol Biol, 2007. 36(2): p. 147-51.

195. Kuipers, I., et al., Increased glutaredoxin-1 and decreased protein S-glutathionylation in sputum of asthmatics. Eur Respir J, 2013. 41(2): p. 469-72.

196. Reynaert, N.L., et al., Dynamic redox control of NF-kappaB through glutaredoxin-regulated Sglutathionylation of inhibitory kappaB kinase beta. Proc Natl Acad Sci U S A, 2006. 103(35): p. 13086-91.

197. Reynaert, N.L., et al., Nitric oxide represses inhibitory kappaB kinase through S-nitrosylation. Proc Natl Acad Sci U S A, 2004. 101(24): p. 8945-50.

198. Strange, R.C., et al., Glutathione-S-transferase family of enzymes. Mutat Res, 2001. 482(1-2): p. 21-6.

199. Kitahata, Y., et al., Prolonged culture of mast cells with high-glucose medium enhances the Fc epsilon RI-mediated degranulation response and leukotriene C4 production. Int Arch Allergy Immunol, 2010. 152 Suppl 1: p. 22-31.

200. Everts, B., et al., TLR-driven early glycolytic reprogramming via the kinases TBK1-IKKE supports the anabolic demands of dendritic cell activation. Nat Immunol, 2014. 15(4): p. 323-32. 
201. Meijer, M., G.T. Rijkers, and F.J. van Overveld, Neutrophils and emerging targets for treatment in chronic obstructive pulmonary disease. Expert Rev Clin Immunol, 2013. 9(11): p. 1055-68.

202. Arora, S., et al., Macrophages: Their role, activation and polarization in pulmonary diseases. Immunobiology, 2018. 223(4-5): p. 383-396.

203. Krawczyk, C.M., et al., Toll-like receptor-induced changes in glycolytic metabolism regulate dendritic cell activation. Blood, 2010. 115(23): p. 4742-9.

204. Li, Q., et al., E3 Ligase VHL Promotes Group 2 Innate Lymphoid Cell Maturation and Function via Glycolysis Inhibition and Induction of Interleukin-33 Receptor. Immunity, 2018. 48(2): p. 258270.e5.

205. Ryu, H., et al., Regulation of M2-type pyruvate kinase mediated by the high-affinity IgE receptors is required for mast cell degranulation. Br J Pharmacol, 2008. 154(5): p. 1035-46.

206. Xu, W., et al., Increased mitochondrial arginine metabolism supports bioenergetics in asthma. J Clin Invest, 2016. 126(7): p. 2465-81.

207. Winnica, D., et al., Bioenergetic Differences in the Airway Epithelium of Lean Versus Obese Asthmatics Are Driven by Nitric Oxide and Reflected in Circulating Platelets. Antioxid Redox Signal, 2019. 31(10): p. 673-686. 
CHAPTER 2: THESIS OUTLINE 
It is now well recognized that oxidants, via redox sensitive processes, control several physiological functions, including cell proliferation, migration, mitochondrial function, and energy metabolism. The highly abundant antioxidant molecule glutathione (GSH) plays a critical role in the effector function of T-lymphocytes. In addition, enhanced glycolysis has been shown to promote growth and survival, through increasing GSH levels to combat the oxidative environment, in multiple cell types including cancer cells. Similarly, it has been demonstrated that the metabolic signature of inflammatory cells can modulate their pro-inflammatory phenotype. For example, the glycolytic metabolite phosphoenolpyruvate's (PEP) accumulation has been shown to promote the inflammatory phenotype of macrophages by increasing the secretion of proinflammatory cytokines [1]. Lactate, however, was reported to suppress the immune response by blocking proinflammatory signaling pathways in monocytes, macrophages and DCs [2,3]. On the other hand, some inflammatory signals such as NF-KB and IL-6 have also been demonstrated to increase glycolysis [4, 5]. As mentioned earlier, some studies have reported a potential link between changes in cellular metabolism and subsequently enhanced inflammation in pathogenesis of asthma. However, it is not fully clear how changes in redox biochemistry and cellular metabolism affect lung epithelial cells and whether this paradigm plays a role in the development of allergic lung diseases. Thus, the overall aim of this thesis was to study the role played by metabolic reprogramming in the development of allergic airways disease and whether this is controlled by redox perturbations.

The use of in vivo animal models is an invaluable tool in studying complex diseases like asthma. This allows for better understanding of the disease pathophysiology, mechanism, and help evaluate both efficacy and safety of treatments before introducing them to patients. Mice, the most commonly used animal in biomedical research, do not develop asthma spontaneously and require a sensitization and re-exposure protocol to elicit the allergic response. Injection of ovalbumin (OVA) along with an adjuvant, followed by intranasal or aerosolized OVA exposure have been used for years as an asthma mouse model. However, some drawbacks to this model exist, and this include: (1) OVA is injected intraperitoneal whereas asthmatic patients are sensitized by inhalation. (2) Prolonged exposure to OVA develops immunological tolerance [6]. (3) OVA-induced immunologic response is primarily of the Th2 type with eosinophil but not neutrophil influx [7]. (4) OVA is not considered one of the common asthma triggers. For these reasons, allergens such as ragweed extract, cockroach antigens and house dust mite extract (HDM), that can be directly relevant to human asthma are now more acceptable [8-10]. 
Approximately $50-85 \%$ of asthmatics are allergic to HDM, making it the most common allergen used in modeling asthma nowadays [10]. The allergic airways disease induced by HDM is largely dependent on the epithelial cells [11] and the production of the proinflammatory cytokines TSLP, GM-CSF, IL-25 and IL-33 [12]. In addition, HDM exposure leads to both eosinophilic and neutrophilic immunologic response, airway inflammation, along with other characteristics of fibrotic remodeling such as smooth muscle hypertrophy, subepithelial fibrosis and mucous hyperplasia [13]. In this thesis, HDM was used as model of allergic airways disease in mice.

Growing body of evidence point at the role played by glycolysis in the development of inflammation. Given that chronic inflammation is an underlying cause of asthma pathogenesis, in chapter $\mathbf{3}$ we investigate whether glycolysis is altered and the significance of these alterations in allergic asthma. In addition to our in vivo studies in mice, we used samples (nasal cells and sputum) derived from asthmatics, as well as mouse tracheal epithelial cells to confirm our findings. In this chapter, we highlight the involvement of enhanced glycolysis in the development of allergic airways disease. We specifically demonstrate that interleukin-1B (IL1B) is the main driver of the enhanced glycolysis during the disease progression. In addition, we demonstrate that enhanced glycolysis and IL1B induce pro-inflammatory cytokines, notably TSLP and GM-CSF, contribute to allergic airways disease. Moreover, we propose increases in lactate and IL1B as biomarkers for severe asthma subtype.

In chapter 4, we dive deeper in studying the glycolysis-induced inflammatory responses in the settings of allergic airways disease. Specifically, we focus on PKM2, the enzyme responsible for catalyzing the last step of glycolysis, and its role in the disease progression. We indeed demonstrate that PKM2 activation attenuates the secretion of several IL1B-induced proinflammatory mediators, decreases mucus metaplasia and collagen deposition during allergic airways disease. Together, proposing PKM2 as a potential target for asthma therapeutics.

The link between metabolic reprogramming and redox homeostasis has been described earlier whereby glycolysis, through PPP, provides NADPH reducing equivalents necessary for redox homeostasis. In chapter $\mathbf{5}$, we use unbiased approaches to study whether and how changes in the redox environment, specifically in protein S-glutathionylation, regulate metabolic reprogramming in lung epithelial cells. Utilizing multi-omics platforms, that include Sglutathionylated proteome, metabolome, and DNA array, we demonstrate that IL1B induces Sglutathionylation of multiple proteins involved in metabolic regulation. Furthermore, cells 
exhibiting higher basal level of S-glutathionylation ( $\left.G / r x^{-/}\right)$have a modulated metabolic profile, exhibit an enhanced IL1B-induced glycolytic reprograming and secrete higher amounts of TSLP, a key cytokine in asthma. This chapter provides an intriguing link between S-glutathionylation chemistry and glycolytic reprogramming in epithelial cells.

The antioxidant glutathione is at the core of the redox system. Our multi-omics study performed in chapter 5 highlighted increases in GSH in response to IL1B stimulation, a scenario similar to the observations made in cancer cells where increases in glycolysis leads to the upregulation of GSH. In addition, our data in the previous chapter point at multiple proteins being targets of S-glutathionylation in response to IL1B. Therefore, in chapter 6, we asked whether Sglutathionylation chemistry plays a role in IL1B-induced GSH increases in epithelial cells and whether this has any functional relevance on inflammatory signaling. Our results here show that indeed S-glutathionylation of the deubiquitinase OTUB1 stabilizes the cystine transporter system $\mathrm{x}_{c}{ }^{-}$, a rate limiting step in GSH synthesis, and allows for increases in GSH levels. Inhibition of GSH, interestingly, attenuates proinflammatory cytokine release, notably TSLP. In addition, the outcome of this chapter points at a unique feed-forward regulation of GSH by S-glutathionylation.

Lastly, chapter 7 provides a general discussion of the key findings of the thesis, accompanied by future directions, implications, and limitations. 


\section{REFERENCES:}

1. Ko, C.W., et al., Macrophages with a deletion of the phosphoenolpyruvate carboxykinase 1 (Pck1) gene have a more proinflammatory phenotype. J Biol Chem, 2018. 293(9): p. 3399-3409.

2. Errea, A., et al., Lactate Inhibits the Pro-Inflammatory Response and Metabolic Reprogramming in Murine Macrophages in a GPR81-Independent Manner. PLoS One, 2016. 11(11): p. e0163694.

3. Hoque, R., et al., Lactate reduces liver and pancreatic injury in Toll-like receptor- and inflammasome-mediated inflammation via GPR81-mediated suppression of innate immunity. Gastroenterology, 2014. 146(7): p. 1763-74.

4. Kawauchi, K., et al., p53 regulates glucose metabolism through an IKK-NF-kappaB pathway and inhibits cell transformation. Nat Cell Biol, 2008. 10(5): p. 611-8.

5. Ando, M., et al., Interleukin 6 enhances glycolysis through expression of the glycolytic enzymes hexokinase 2 and 6-phosphofructo-2-kinase/fructose-2,6-bisphosphatase-3. J Nippon Med Sch, 2010. 77(2): p. 97-105.

6. Yiamouyiannis, C.A., et al., Shifts in lung lymphocyte profiles correlate with the sequential development of acute allergic and chronic tolerant stages in a murine asthma model. Am J Pathol, 1999. 154(6): p. 1911-21.

7. Schramm, C.M., et al., Chronic inhaled ovalbumin exposure induces antigen-dependent but not antigen-specific inhalational tolerance in a murine model of allergic airway disease. Am J Pathol, 2004. 164(1): p. 295-304.

8. Wild, J.S., et al., IFN-gamma-inducing factor (IL-18) increases allergic sensitization, serum IgE, Th2 cytokines, and airway eosinophilia in a mouse model of allergic asthma. J Immunol, 2000. 164(5): p. 2701-10.

9. Kim, J., et al., Eotaxin represents the principal eosinophil chemoattractant in a novel murine asthma model induced by house dust containing cockroach allergens. J Immunol, 2001. 167(5): p. 2808-15.

10. Nelson, R.P., Jr., et al., Allergen-specific IgE levels and mite allergen exposure in children with acute asthma first seen in an emergency department and in nonasthmatic control subjects. J Allergy Clin Immunol, 1996. 98(2): p. 258-63.

11. Gregory, L.G. and C.M. Lloyd, Orchestrating house dust mite-associated allergy in the lung. Trends Immunol, 2011. 32(9): p. 402-11.

12. Hammad, H., et al., House dust mite allergen induces asthma via Toll-like receptor 4 triggering of airway structural cells. Nat Med, 2009. 15(4): p. 410-6.

13. Tully, J.E., et al., Epithelial NF-KB orchestrates house dust mite-induced airway inflammation, hyperresponsiveness, and fibrotic remodeling. J Immunol, 2013. 191(12): p. 5811-21. 


\section{CHAPTER 3:}

IL-1/inhibitory KB kinase $\varepsilon$-induced glycolysis augment epithelial effector function and promote allergic airways disease

Qian X, Aboushousha $\mathrm{R}^{*}$, van de Wetering $\mathrm{C}^{\star}$, Chia SB, Amiel E, Schneider RW, van der Velden JLJ, Lahue KG, Hoagland DA, Casey DT, Daphtary N, Ather JL, Randall MJ, Aliyeva M, Black KE, Chapman DG, Lundblad LKA, McMillan DH, Dixon AE, Anathy V, Irvin CG, Poynter ME, Wouters EFM, Vacek PM, Henket M, Schleich F, Louis R, van der Vliet A, JanssenHeininger YMW. * equal contribution Journal of Allergy and Clinical Immunology 2018 Aug;142(2):435-450.e10. 


\section{Abstract}

\section{Background:}

Emerging studies suggest that enhanced glycolysis accompanies inflammatory responses. Virtually nothing is known about the relevance of glycolysis in allergic asthma.

\section{Objectives:}

Here we sought to determine if glycolysis is altered in allergic asthma and to address its importance in the pathogenesis of allergic asthma.

\section{Methods:}

We examined alterations in glycolysis in sputum samples from asthmatics and primary human nasal cells, and used murine models of allergic asthma as well as primary mouse tracheal epithelial cells to evaluate the relevance of glycolysis.

\section{Results:}

In a murine model of allergic asthma, glycolysis was induced in the lungs in an IL-1-dependent manner. Furthermore, administration of IL-1 $\beta$ into airways stimulated lactate production and expression of glycolytic enzymes, with notable expression of lactate dehydrogenase A occurring in the airway epithelium. Indeed, exposure of mouse tracheal epithelial cells to IL-1 $\beta$ or IL-1 $\alpha$ resulted in increased glycolytic flux, glucose usage, expression of glycolysis genes, and lactate

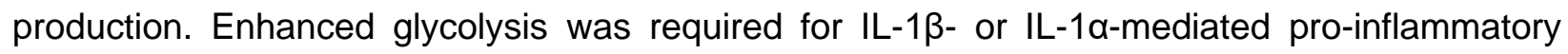
responses and the stimulatory effects of IL-1 $\beta$ on HDM-induced release of TSLP, and GM-CSF from tracheal epithelial cells. Inhibitor of $\mathrm{KB}$ kinase $\varepsilon$ was downstream of house dust mite (HDM) or IL-1 $\beta$, and was required for HDM-induced glycolysis and the pathogenesis of allergic airways disease. SiRNA-ablation of lactate dehydrogenase A attenuated HDM-induced increases in lactate and attenuated HDM-induced disease. Primary nasal epithelial cells from asthmatics intrinsically produced more lactate as compared to cells from healthy subjects. Lactate content was significantly higher in sputum supernatants from asthmatics, notably those patients with $>61 \%$ neutrophils. A positively correlation was observed between sputum lactate and IL-1 $1 \beta$, and lactate content negatively correlated with lung function.

\section{Conclusions:}

Collectively, these findings demonstrate that IL-1 $\beta / I K K \varepsilon$ signaling plays an important role in HDMinduced glycolysis and the pathogenesis of allergic airways disease. 
Keywords: Asthma, house dust mite, glycolysis, interleukin-1, inhibitor of $\mathrm{kB}$ kinase $\varepsilon$, lactate, lactate dehydrogenase $\mathrm{A}$

\section{Capsule summary:}

IL-1 and IKKE play important roles in HDM-induced glycolysis and the pathogenesis of allergic airways disease, and lactate is a potential biomarker for increased glycolysis and IL-1-associated pro-inflammatory signals in airways of asthmatics.

\section{Graphical Abstract}

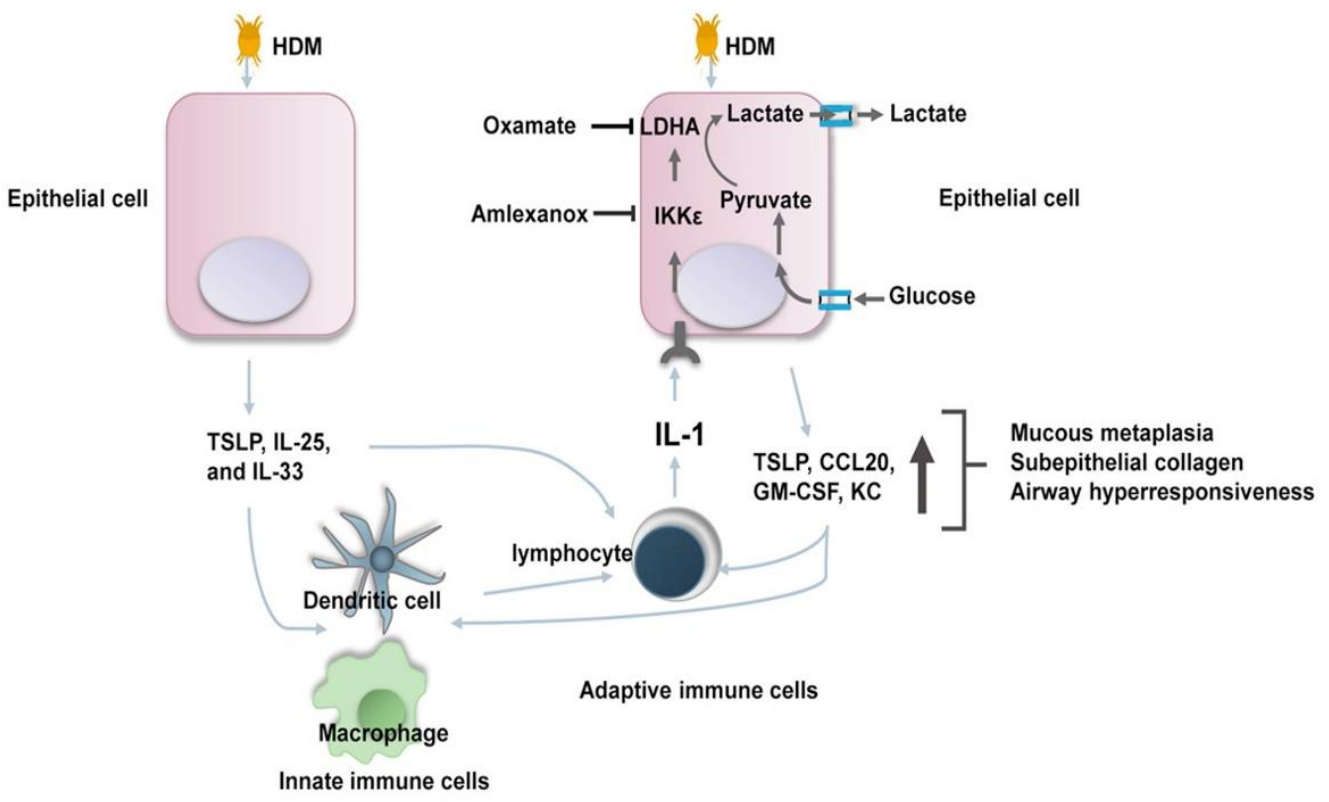

Abbreviations used: HDM; house dust mite, TSLP; Thymic stromal lymphopoietin, IL; Interleukin, IKKE; I kappa B Kinase epsilon, LDHA; lactate dehydrogenase A, GM-CSF; Granulocyte-macrophage colony-stimulating factor, KC; Chemokine (C-X-C motif) ligand 1, CCL20; Chemokine (C-C motif) ligand 20 


\section{Introduction}

Asthma is a pulmonary disorder that is characterized by reversible airflow obstruction, chronic airway inflammation, airways hyperresponsiveness (AHR) and remodeling. Asthma affects nearly $10 \%$ of the US population and is increasing in prevalence, making it a major public health problem (1). Asthma is a complex and heterogeneous syndrome and has a number of different clinical phenotypes that are associated with distinct cellular and molecular mechanisms (2) controlled by innate and adaptive immune responses to allergens, which rely on both immune (such as DCs, Th2 and Th17 cells, and innate lymphoid cells) and structural cells that include airway epithelium $(3,4)$. The exact biochemical processes underlying the diverse phenotypes of asthma, and the precise contributions of lung structural and immune cells during asthma pathogenesis remain incompletely understood.

Changes in cellular metabolism, notably increases in glycolysis, accompany inflammatory responses (5). Glucose is taken up by cells through glucose transporters and subsequently undergoes glycolysis via a step-wise cascade to form pyruvate that can enter the mitochondria and undergo oxidative phosphorylation. Alternatively, pyruvate can be metabolized to lactate, via lactate dehydrogenase (LDHA). Aerobic glycolysis, the metabolism of glucose to form lactate in the presence of oxygen, is a feature of tumor or metabolically active cells, and is associated with increased glucose uptake and lactate over-production (6, 7). Aerobic glycolysis also generates $\mathrm{NADPH}$ that is important in protection against oxidative stress, and preserves the carbon backbone of glucose to fuel the synthesis of macromolecules $(6,7)$.

Glucose metabolism is implicated in immune activation, and increases in glycolysis regulate immune effector function through multiple mechanisms (8). For example, enhanced glycolysis has been shown to facilitate the polarization and/or activation of immune cells (9). Moreover, lactate, the end product of glycolysis, accumulates at the sites of chronic inflammation $(10,11)$, and tumor microenvironments (12), indicative of increased glycolytic flux. Virtually nothing, however, is known about the glycolytic status in the setting of, and the relevance of deregulated glycolysis in the pathogenesis of allergic airways diseases. A previous study demonstrated increases in lactate in serum of asthmatics compared to patients with COPD or healthy controls, and increases in lactate in proliferating CD4 T cells isolated from asthmatics compared to healthy subjects. The same authors demonstrated that intraperitoneal injection of dichloroacetate, an 
inhibitor of pyruvate dehydrogenase kinase, attenuated increases in lactate in proliferating CD4 $T$ cells, and attenuated ragweed-induced allergic airways inflammation and airways hyperresponsiveness in mice (13). However, the extent of increases in glycolysis in airways of asthmatics remain unknown. Similarly, the signals that promote increases glycolysis in allergically-inflamed lung tissue also remain elusive. It also is not clear whether increases in glycolysis occur in lung epithelial cells and affects the response of epithelial cells to house dust mite allergen. Therefore, the goal of the current study was to address some of these questions, using a mouse model of house dust mite (HDM)-induced allergic airways disease, nasal epithelial cells and sputum samples derived from asthmatics. Our results demonstrate that increases in glycolysis are a critical feature of allergic airways disease, controlled by an IL-1//KKE signaling axis.

\section{Materials and Methods}

\section{Subject characteristics}

The study population was enrolled at the asthma clinic in CHU Liege (Belgium). Healthy subjects were recruited at the hospital and University of Liege, Belgium. The study cohort consisted of healthy subjects $(n=20)$ and patients with asthma $(n=94)$. The demographic and functional characteristics of the 114 subjects from the study cohort are shown in Table E1. The study was approved by the local ethics committee, University of Liege, Belgium, (reference 2005/181; conforming to the declaration of Helsinki).

Nasal epithelial cells were isolated from healthy subjects $(n=6)$ or patients with allergic rhinitis and asthma asthmatics $(n=7)$ enrolled at the University of Vermont Medical Center. Patient characteristics are provided in Table E2. The local IRB granted approval for all of the procedures involving human subjects (CHRMS 15-067).

Additional details are provided as Online Supplementary Information.

\section{Mouse studies}

Age-matched, 8- to 12-week-old mice were used (The Jackson Laboratory, Bar Harbor, ME) for all experiments. Wild-type (WT, C57BL6/NJ)), Rag $^{-/-}$(C57BL6/J), or lkbke ${ }^{-/-}$(C57BL6/J) mice along with their strain-matched controls were sensitized (Days 1 and 8), challenged (Days 1519), and rechallenged (Days 29, 32, 36, and 39) with HDM extract as shown in Figure 1A. All animal experiments were approved by the Institutional Animal Care and Use Committee.

\section{Cell studies}


Human nasal epithelial cells were isolated from healthy subjects or asthmatics. Cells were cultured and exposed to HDM for assessment of glycolysis proteins and lactate content in culture supernatants. Mouse tracheal epithelial cells were isolated from tracheas from WT mice of mice or mice lacking lkbke. Cells were cultured and exposed to the indicated mediators, for the assessment of lactate in supernatants, glucose uptake, extracellular acidification rate, and cytokine levels in medium.

\section{Statistical analysis}

All data were evaluated using JMP Pro 10 software (SAS Institute, Cary, NC) and GraphPad Prism 6 (GraphPad Software, Inc., La Jolla, CA). Cell culture and mouse data were compared with either one-way, or two-way ANOVA, followed by a Tukey post hoc test. Scoring of histological staining was analyzed by the Kruskal-Wallis test. Human demographic data were compared by Student T-test, Chi-squared test or Wilcoxon rank sum test. Human sputum data for lactate and IL-1 $\beta$ were log-transformed before being compared by Student T-tests. Comparisons of sputum data between asthmatic and healthy participants were adjusted for differences in BMI using ANCOVA. $P$ values less than 0.05 were considered statistically significant.

More detailed information on the materials and methods used in this study is available as supplemental information. 


\section{Results}

\section{Increases in glycolysis in lungs from mice with house dust mite (HDM)-induced allergic airways disease.}

Low $\mathrm{pH}$ is a characteristic of chronic inflammatory sites $(10,14,15)$ and results mainly from a metabolic shift to aerobic glycolysis and subsequent lactate over-production. Little is known about the glycolytic status in asthma. We therefore first determined whether glycolysis was affected in a HDM model of allergic airways disease (Figure 1A). No increases in lactate were observed acutely following HDM (Day 1 and 2, Figure 1B). Five consecutive daily exposures to HDM in week 3 without prior sensitization during week 1 and 2 (day 20: 2X saline, 5X HDM) also did not result in increases in lactate (Figure 1B). However, lactate levels were increased in the BAL and lung tissue homogenates of mice at Day 20 following 2 sensitizations and 5 challenges (Figure 1B). Significant increases in lactate production were also observed $24 \mathrm{~h}$ (Day 30) following HDM re-challenge on day 29 , in mice previously sensitized and challenged with HDM (2XHDM, 5X HDM, HDM). These increases in lactate on days 20 and 30 corresponded with increases in total cells and notably increases in neutrophils in BAL (16) (Figure E1 A and B) and suggest that increases in lactate are a feature of the adaptive immune response. Increased expression of glycolysis proteins, including hexokinase 1 (HK1), HK2, and lactate dehydrogenase A (LDHA) were observed in lung tissue homogenates 20 or 29 days post HDM exposure, while they tended to decrease at day 30 (Figure 1C). LDHA preferentially converts pyruvate to lactate (17). Immunohistochemical analysis of LDHA in saline-exposed mouse lung tissues revealed that LDHA was constitutively expressed in bronchial epithelial and alveolar type II cells (Figure 1D). In response to HDM sensitization and challenge, widespread increases in expression of LDHA were apparent in lung tissue, with increases in immunoreactivity present not only in cells resembling infiltrating immune cells, consistent with the previously appreciated role of glycolysis in immune effector function (9), but also in bronchial epithelial cells (Figure 1D). These findings suggest that both structural and hematopoietic cells might be responsible for HDM-mediated increases in lactate production. 

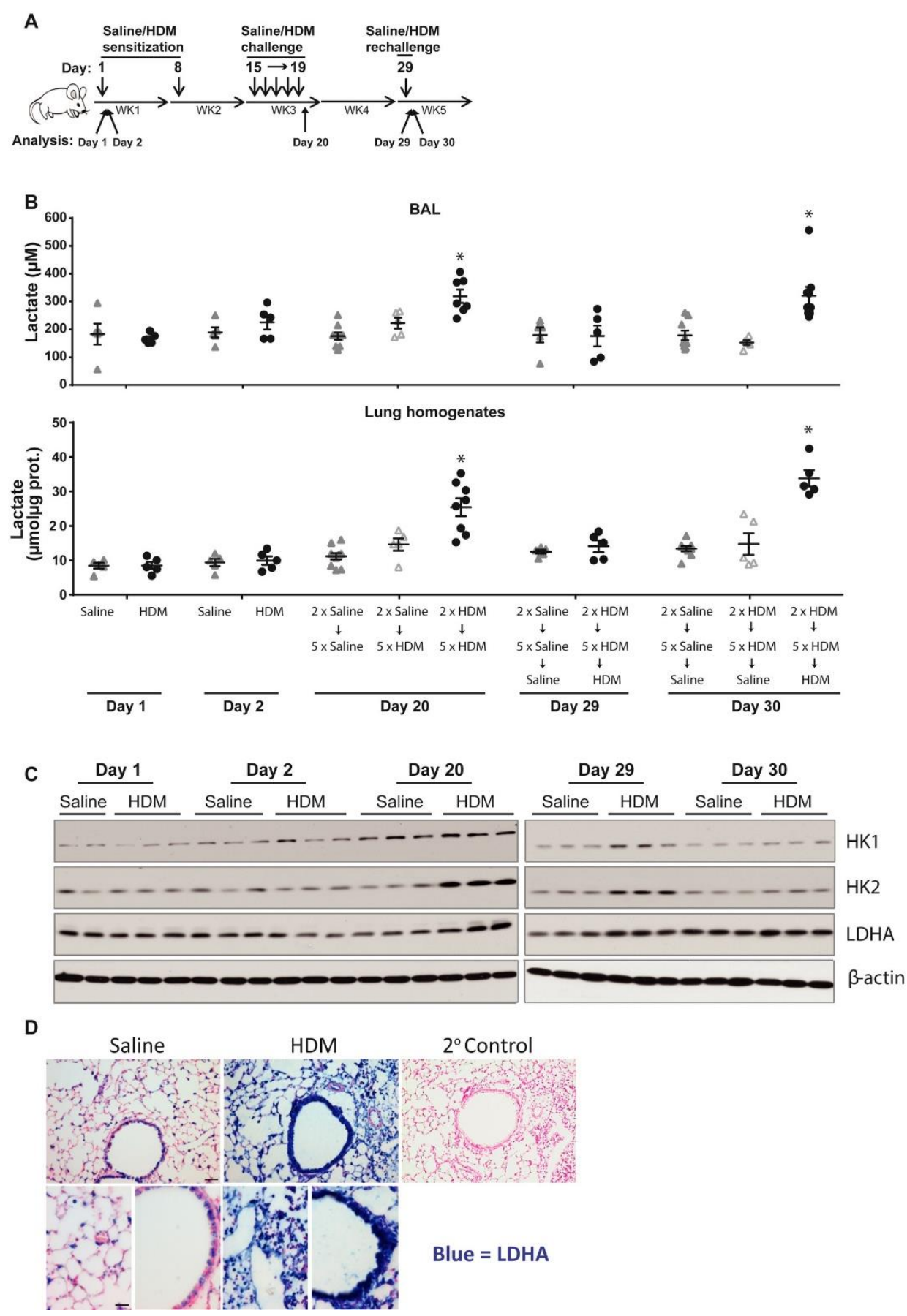

Figure 1. Evaluation of glycolysis in the lung tissues of mice exposed to house dust mite (HDM). A, Schematic depicting the dosing regimen of HDM (Detailed information is provided in the Supplemental Material). B, Lactate levels in BAL (top) and lung tissues (bottom) following a single or multiple exposures to HDM, according to the schematic in $A$. ${ }^{*} P<0.05$ (ANOVA) relative to the saline group ( $n=5-8$ per group). C, Protein expression of glycolysis enzymes in lung lysates from saline- or HDM-challenged mice harvested 
at the indicated times. $\beta$-Actin = loading control. D, LDHA immunohistochemistry in lung tissues of HDMsensitized and -challenged mice harvested at Day 20 (Top: scale bar, $50 \mu \mathrm{m}$; Bottom: scale bar, $25 \mu \mathrm{m}$ ). Blue $=$ LDHA. $2^{\circ}$ control; HDM-inflamed tissue wherein primary antibody was omitted as a negative control.

\section{An adaptive immune response and Interleukin-1 (IL1) signaling are required for increases in glycolysis in lungs of mice with HDM-induced allergic airways disease:}

To elucidate the mediators that cause glycolysis, we evaluated a number of proinflammatory mediators and assessed whether their levels correlated with increases in lactate. Levels of IL-1 $\mathrm{IL}-6$, and TNF $\alpha$ but not IL-1 $\alpha$ and IL-17 were increased at times that roughly corresponded with increases in lactate (Figure $2 \mathrm{~A}$ ). We next sought to determine whether IL-1 signaling plays a causal role in the augmentation of glycolysis in HDM-induced disease. Neutralization of IL-1 with IL-1 trap (18) (Figure E2) attenuated the HDM-mediated lactate increases (Figure 2B) as well as expression of glycolytic enzymes HK2 and LDHA (Figure 2C), demonstrating the functional importance of IL-1 in augmenting glycolysis in HDM-exposed mice.

The delayed increases in lactate in BAL and lung tissues following HDM sensitization and challenge suggest the requirement of an adaptive immune response. To directly test whether IL1-dependent increases in glycolysis in response to HDM were dependent on adaptive immunity, we assessed lactate levels in HDM-exposed WT and Rag $T^{-/-}$mice which lack mature B and T lymphocytes (19). We previously published that $R a g 1^{-/-}$mice exhibited robust decreases in HDMinduced immune cell influx in BAL and IgG and IgE production (20). Strikingly, the HDM-mediated increases in lactate levels (Figure 2D) and IL-1 $\beta$ (Figure 2E) were completely inhibited in $\mathrm{Rag}^{-/-}$ mice. Taken together, our results suggest that HDM-induced adaptive immunity is required for IL1 signaling and resultant increases in glycolysis. In order to address whether IL-1 $\beta$ is sufficient to increase glycolysis, we directly administrated IL-1 into the airways of WT mice. IL-1 $\beta$ caused increases in lactate levels in BAL at 6 and $24 \mathrm{~h}$ post administration, and in lung tissue after $48 \mathrm{~h}$ post administration, and resulted in increases in BAL neutrophils, along with increases in the proinflammatory cytokines, CCL20, KC, GM-CSF and TSLP in lung tissue (Figure E3A-C). Increases in lactate were accompanied by increases in HK2 and LDHA in lung tissue (Figure 2G). Evaluation of LDHA by immunohistochemistry revealed increases in LDHA in bronchial epithelia $24 \mathrm{~h}$ postadministration of IL-1 $\beta$ (Figure $2 \mathrm{H}$ ). 

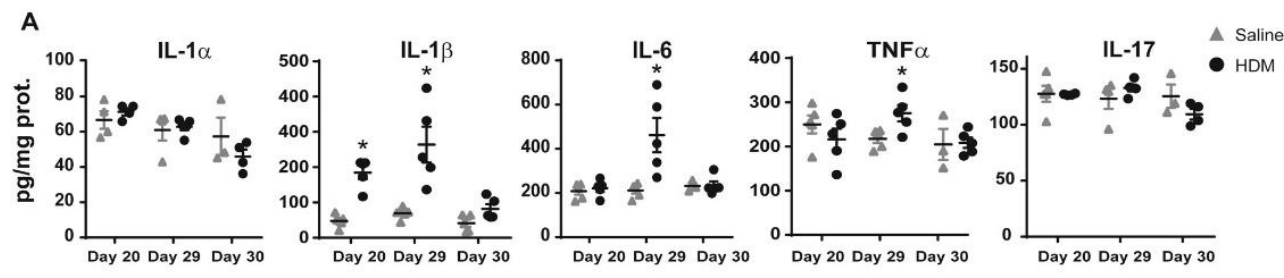

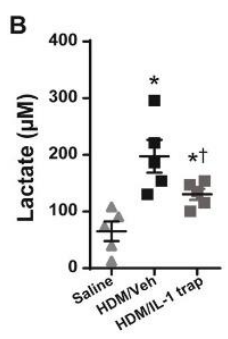

D
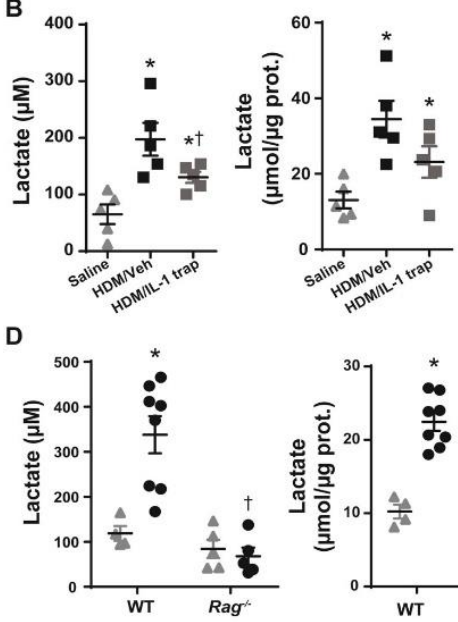

C
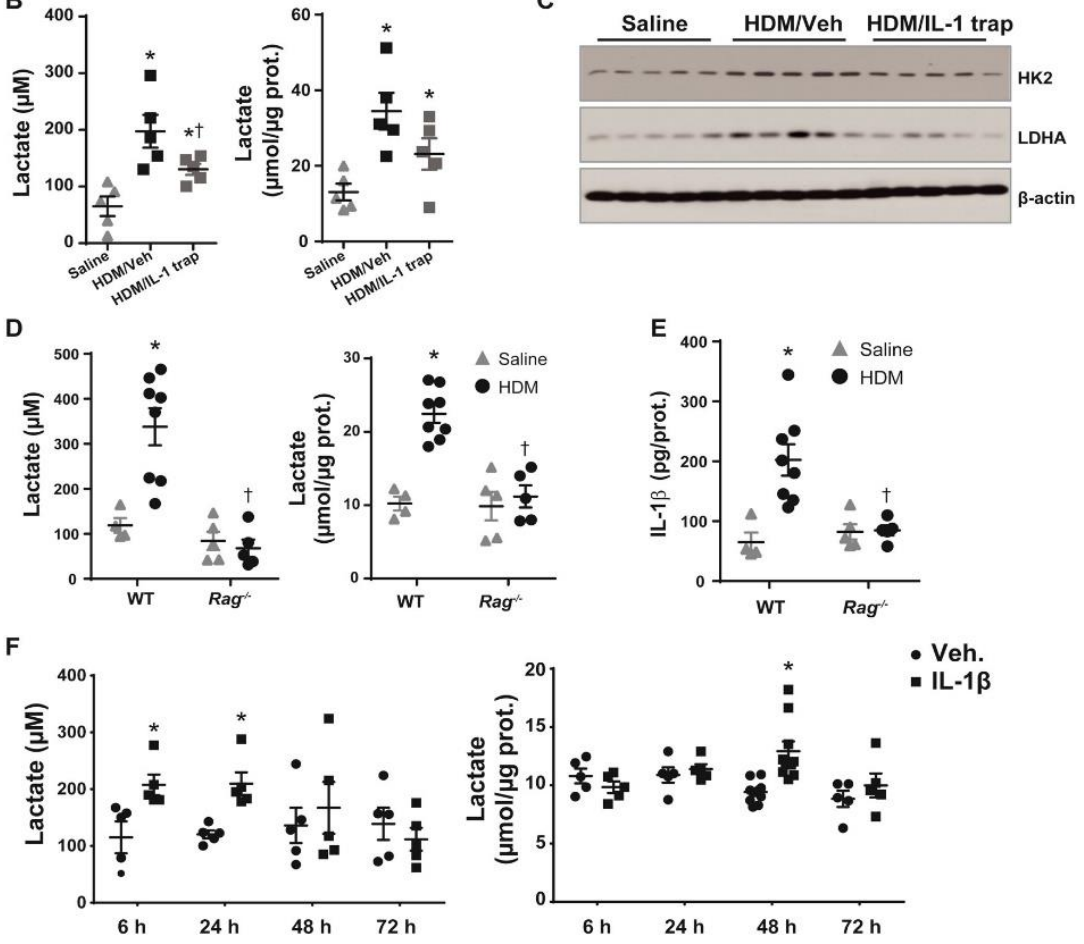

G

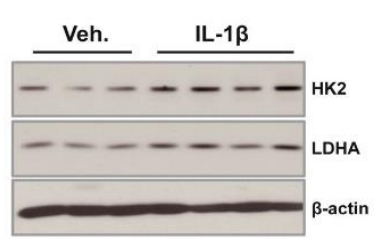

H

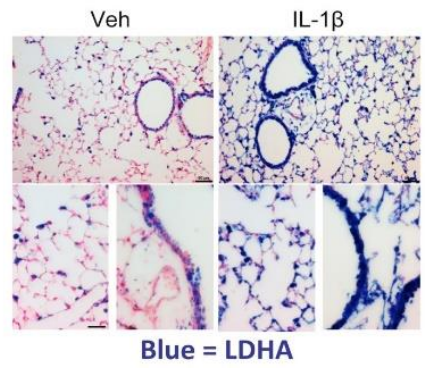

Figure 2. House dust mite (HDM)-induced T and B-cell adaptive immune responses are required for IL-1 $\beta$ production and resultant increases in glycolysis in lung tissues. A, Levels of pro-inflammatory cytokines in lung tissue of HDM-exposed mice at the times indicated. ${ }^{*} P<0.05$ compared to saline groups (ANOVA) ( $n=5$ per group). B-C, Lactate levels in the broncho-alveolar lavage fluid (BAL) and homogenized lung tissues (B) and Western blot analysis of HK2 and LDHA in lung tissues (C) from saline-exposed mice or HDM-exposed mice treated with vehicle (Veh) or IL-1 TRAP. Mice were harvested at day $20 .{ }^{*} P<0.05$ compared to the saline group, $\dagger P<0.05$ compared to the HDM/Veh group (ANOVA) ( $n=5$ per group). Lactate levels in BAL fluid and lung tissues (D) and IL-1 $\beta$ levels in the lung tissues (E) from $\mathrm{Rag}^{-/}$mice and WT mice exposed to saline or HDM. Mice were analyzed at Day 20. ${ }^{*} P<0.05$ compared to the saline 
controls, $† P<0.05$ compared to the respective WT group (ANOVA, $n=4-8$ per group). $F$, Lactate levels in BAL fluid and lung tissues from the mice $6,24,48$, and $72 \mathrm{~h}$ post intranasal administration of IL-1 $3 .{ }^{*} P<$ 0.05 compared to Veh-exposed mice (ANOVA, $n=5-8$ per group). G, Western blotting of HK2 and LDHA in lung tissues from mice treated with recombinant IL-1 $\beta$ ( $1 \mu \mathrm{g} /$ mouse) for $48 \mathrm{~h}$. $\mathbf{H}$, Immunohistochemical analysis of LDHA in lung tissues $24 \mathrm{~h}$ post administration of IL-1 $\beta$ or vehicle (Top: scale bar, $50 \mu \mathrm{m}$; Bottom: scale bar, $25 \mu \mathrm{m})$. Blue = LDHA.

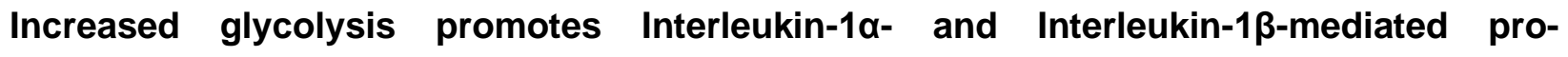 inflammatory responses in airway epithelial cells and augments release of pro- inflammatory mediators following subsequent exposure to house dust mite.}

Our findings demonstrating that intranasal administration of IL-1 $\beta$ increases BAL lactate levels 6 $\mathrm{h}$ later, a time point prior to the recruitment of inflammatory cells (Figures E3B and E3C), suggest that IL-1 $\beta$ increases glycolysis in airway epithelial cells in settings of allergic airways disease. In order to directly address this possibility, we exposed mouse tracheal epithelial (MTE) cells to IL$1 \beta$ or IL-1 $\alpha$ for $24 \mathrm{~h}$. Both cytokines resulted in significant increase in lactate levels in culture supernatants. No increases in lactate were observed $24 \mathrm{~h}$ after exposure to IL-6, IL-13, IL-33, TGF- $\beta 1$, TNF $\alpha$, IL-17, LPS, or HDM (Figure 3A), demonstrating notable selectivity of IL-1 $\alpha / \beta$ in augmenting glycolysis in MTE cells in these experimental settings. Concomitant to increases in lactate, IL-1 $\beta$ significantly augmented expression of a number of genes in the glycolysis pathway (Figure 3B). IL-1 $\beta$-treated MTE cells had higher basal extracellular acidification rates (ECAR) than vehicle-exposed cells, indicating a higher rate of release of lactate into the culture medium. In response to glucose injection, IL-1 $\beta$-treated cells demonstrated higher rate of ECAR compared to control cells. IL-1 $\beta$-treated cells were also more sensitive to the ATP synthesis inhibitor, oligomycin and maintained a higher ECAR (Figure 3C), revealing the higher glycolytic capacity of IL-1 $\beta$ - treated cells. Addition of 2-deoxyglucose (2-DG), a competitive inhibitor of glucose hexokinase, decreased ECAR to baseline levels, confirming that the observed ECAR is due to glycolysis (Figure $3 \mathrm{C}$ ). In contrast to changes in ECAR, oxygen consumption rates (OCR) were similar in control and IL-1 $\beta$-treated cells (Figure $3 \mathrm{C}$ ). In line with these observations, glucose levels in the medium decreased and glucose uptake was increased in response to IL-1 $\beta$ (Figure 3D). These findings collectively demonstrate IL-1 (and IL-1 $\alpha$ ) as an inducer of glycolysis in lung epithelial cells, and that the ability of IL-1 to augment glycolysis in these experimental settings is not shared by other asthma-relevant mediators tested herein. 
The importance of glycolysis in regulating immune effector function responses is well established $(21,22)$. It is not known whether glycolysis regulates pro-inflammatory responses in epithelial cells exposed to IL-1 $\beta$. We therefore inhibited glycolysis by pre-treating the MTE cells with the hexokinase inhibitor, 2-DG, or the LDHA inhibitor, oxamate, and assessed IL-1 $\beta$-induced proinflammatory cytokines. As shown in Figure 4A, 2-DG completely blocked IL-1B-induced lactate production and strongly attenuated production of TSLP, GM-CSF, KC and CCL20 in response to IL-1 $\beta$ (Figure 4B). Similar inhibitory effects on IL-1 $\beta$-induced lactate and pro-inflammatory cytokines were observed in cells treated with oxamate (Figure $4 C$ and D). 2-DG or oxamate did not induce cell death (Figure E4A) demonstrating that decreases in cytokines observed are not due to a loss of survival. Similar to IL-1 $\beta$, IL-1 $\alpha$ also resulted in increases in the same cytokines which were also inhibited by 2-DG or oxamate (Figure E4B), suggesting that both interleukins trigger similar glycolysis-dependent pro-inflammatory responses in epithelial cells.

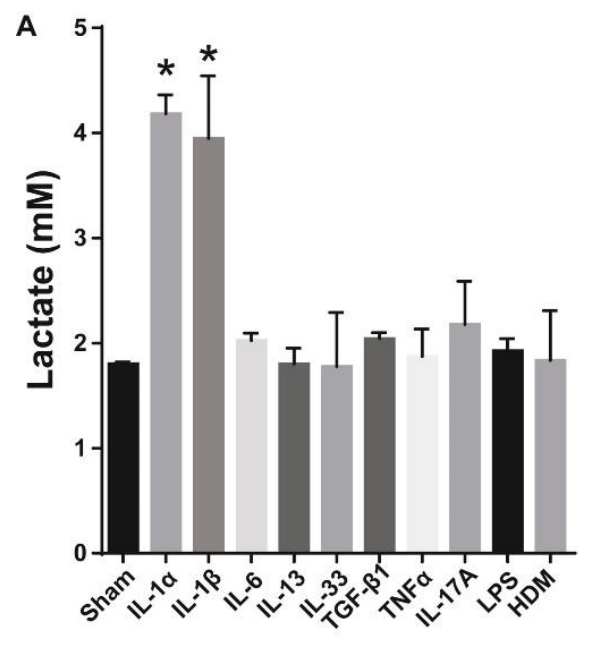

C

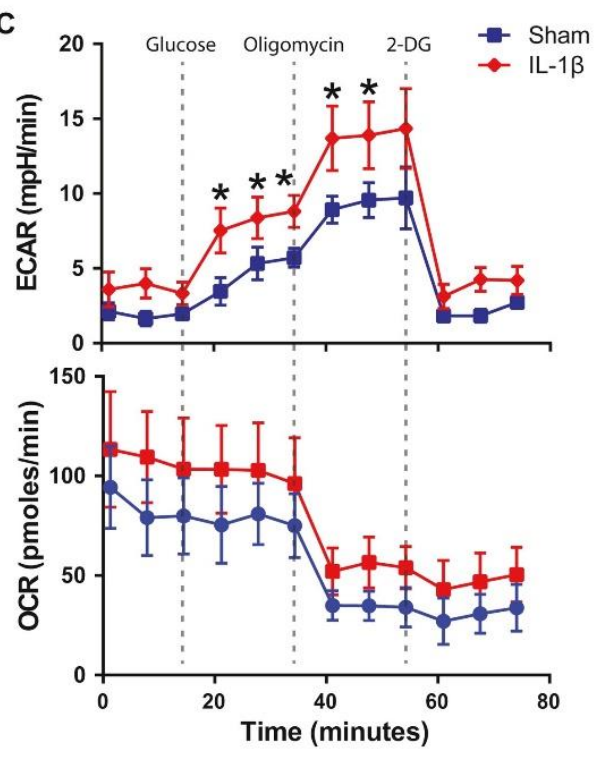

B

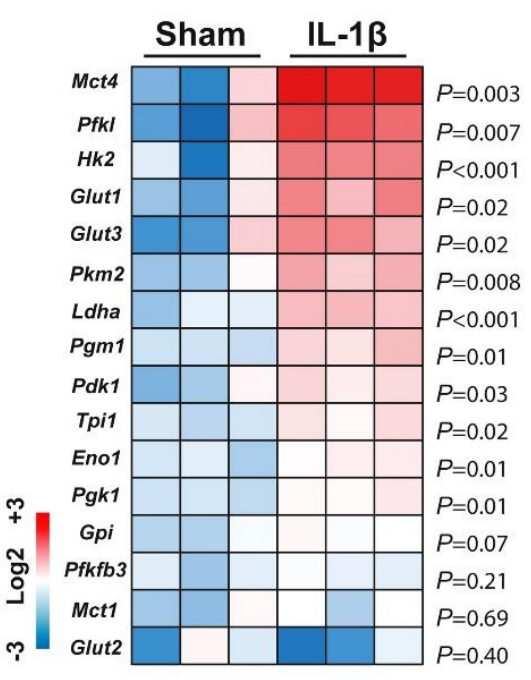

Glycolysis genes
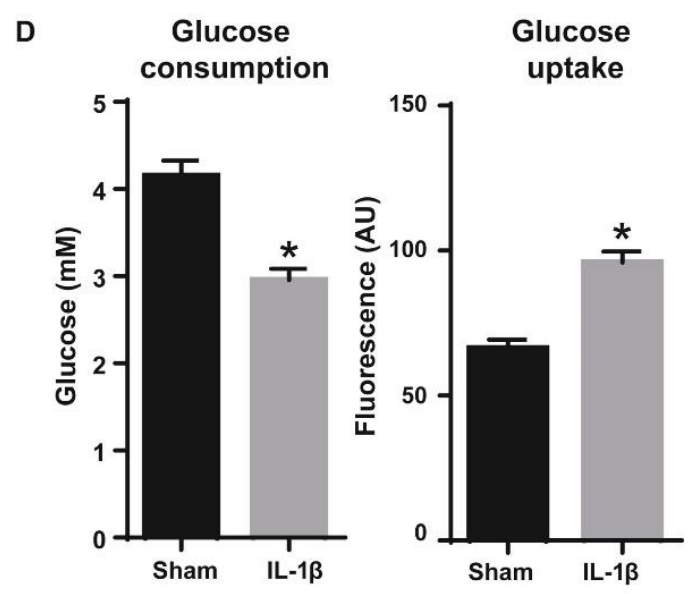
Figure 3. IL-1 $\alpha / \beta$ increase lactate production, glycolysis gene expression, glucose usage, and glycolytic flux rate in primary mouse tracheal epithelial (MTE) cells. A, Lactate levels in the cell-culture supernatants of MTE cells following $24 \mathrm{~h}$ stimulation with IL-1 $\alpha$, IL-1 1 , IL-6, IL-13, IL-33, TGF- $\beta 1$, TNF $\alpha$, IL-17, LPS, or HDM. ${ }^{*} P<0.05$ compared to the sham group (ANOVA). Representative results from one out three independent experiments are shown. B, mRNA expression of glycolysis-related genes in MTE cells treated with or without IL-1 $\beta$ (10 $\mathrm{ng} / \mathrm{mL}$ ). $P$ values from Student's $t$ test are indicated. C, ECAR and OCR of IL-1 $\beta$ - or sham-treated MTE cells, measured via a Seahorse Extracellular Flux (XF24) Analyzer. Glucose, oligomycin, and 2-DG were injected sequentially marked by the vertical lines. ${ }^{*} \mathrm{P}<0.05$ compared to the sham group (Student's $t$ test). Representative results out three independent experiments were shown. D, glucose consumption (left) and uptake (right) in MTE cells $24 \mathrm{~h}$ post stimulation with IL-1 $\beta$. *P $<0.05$ compared to the sham group (Student's $t$ test).

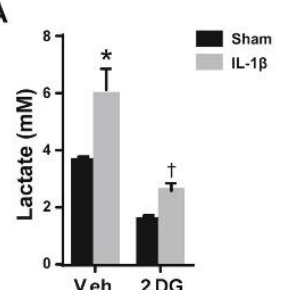

C

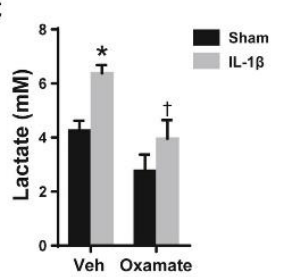

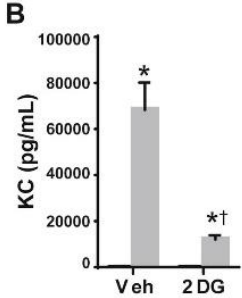

D

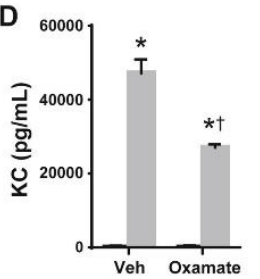

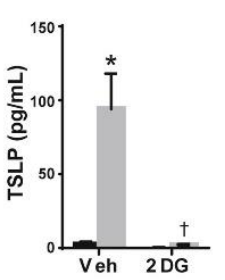

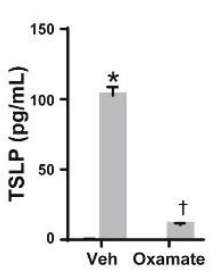

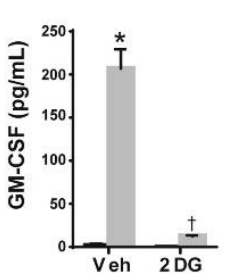
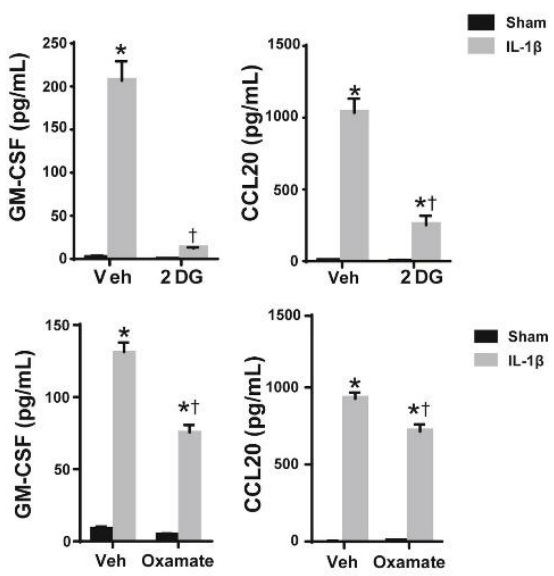

$\mathbf{E}$

$$
\text { MTECs } \rightarrow \text { 2-DG } \rightarrow \mathrm{IL}-1 \beta \rightarrow \mathrm{HDM}
$$

$1 \mathrm{~h}$
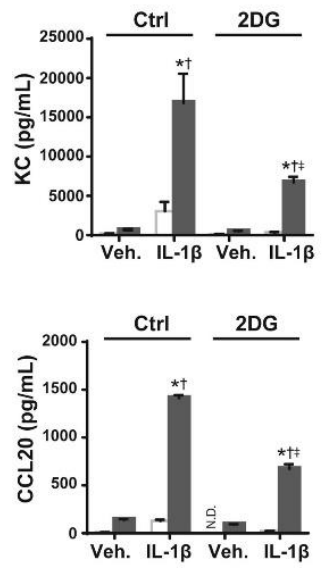

$24 \mathrm{~h} \quad 2 \mathrm{~h}$
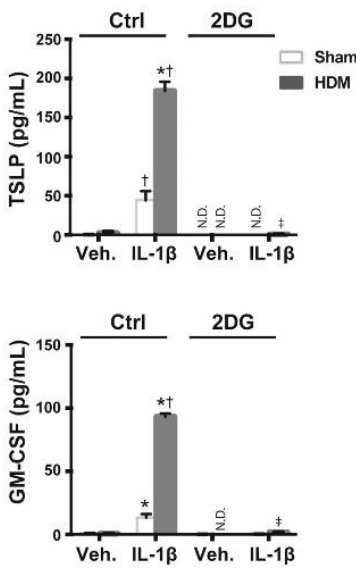

$\mathbf{F}$

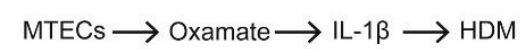

$\mathrm{O} / \mathrm{N}$
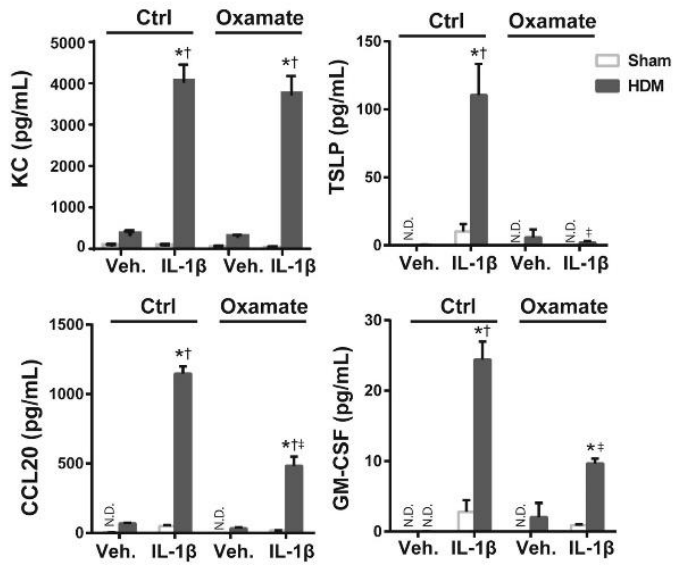

Figure 4. Importance of glycolysis for IL-1 $\beta$-induced pro-inflammatory responses and the IL-1 $\beta$ mediated augmentation of HDM-induced innate cytokine responses in primary mouse tracheal epithelial (MTE) cells. A-D, Lactate (A\&C) and levels of proinflammatory mediators (B\&D) in the cell culture supernatants of MTE cells. MTE cells were pre-treated with 2-Deoxyglucose (2-DG, $10 \mathrm{mM})(\mathbf{A}-\mathbf{B})$, 
or oxamate $(10 \mathrm{mM})(\mathbf{C}-\mathbf{D})$, followed by stimulation with IL-1 $\beta(10 \mathrm{ng} / \mathrm{mL})$ for $24 \mathrm{~h}$. E-F, Importance of glycolysis in the IL-1 $\beta$ - mediated augmentation of HDM $(50 \mu \mathrm{g} / \mathrm{ml})$-induced KC, CCL20, TSLP, and GMCSF levels in culture supernatants. ${ }^{*} P<0.05$ compared to non-HDM exposed sham group, $\dagger P<0.05$ compared to respective non-IL-1 $\beta$ treated vehicle group (Veh.), and $\ddagger P<0.05$ relative to non-2DG or nonoxamate treated control group (Ctrl) (two-way ANOVA).

Results in Figures $2 \mathrm{~F}$ and $\mathrm{H}$ demonstrate that IL1 $\beta$ was sufficient to increase lactate in lung tissues and expression of LDHA in bronchial epithelia. We next tested whether IL-1 $\beta$-mediated increases in glycolysis in epithelial cells affected their subsequent response to HDM, in order to gain insights into the functional impact of enhanced glycolysis (which would be expected to occur in a setting wherein IL-1 is increased), for subsequent responses to allergens in airway epithelia. We treated MTE cells with 2-DG for one $\mathrm{h}$ or oxamate overnight, followed by treatment with IL-1 $\beta$ for $24 \mathrm{~h}$. Cells were then washed and exposed to HDM for $2 \mathrm{~h}$ (Figure 4E and F). While IL-1 $\beta$ or HDM individually led to increases in pro-inflammatory cytokines, a strong synergy was observed in cells sequentially exposed to IL-1 $\beta$ and HDM. Importantly, inhibition of glycolysis with 2-DG strongly attenuated the IL-1 $\beta$ plus HDM induced levels in TSLP or GM-CSF, and moderately decreased CCL-20 and KC (Figure 4E). Similar responses were observed with oxamate (Figure $4 \mathrm{~F}$ ) with the exception of $\mathrm{KC}$ which remained unaffected. Collectively, these data demonstrate that IL-1 $\beta$-induced glycolysis augments the subsequent pro-inflammatory responses of epithelial cells to HDM.

\section{Inhibitory kappa B kinase-epsilon, (IKKع) promotes IL-1 $\beta$-induced glycolysis in epithelial cells and HDM-induced allergic airways disease in mice}

The inhibitory kappa B kinase (IKK) family includes four kinase members, the canonical IKKa and $\mathrm{IKK} \beta$, as well as two non-canonical family members, IKKE and TBK1. We have previously shown that activation of IKK $\beta$ play a critical role in the pathogenesis of allergic airways disease $(23,24)$. Essentially no information exists about the role of other IKKs. IKKE is emerging as a critical regulator of Th17 maintenance, IL-17-induced airway neutrophilia (25), and glycolytic reprogramming in DCs (21). We therefore explored whether IKKE was increased during the pathogenesis of HDM-induced allergic airways disease, and whether IKKE contributed to IL-1 $\beta$ induced glycolysis. In mice with HDM-induced disease, expression of IKKa and IKK $\beta$ increased in lung tissues (Figure 5A), consistent with our previous observations (16, 26). We also observed robust and prolonged increases in IKKE and TBK1 in lung tissues (Figure 5A). We next addressed the impact of Ikbke ablation (the gene encoding IKKE) (Figure 5B) on HDM-induced glycolysis 
and allergic airways disease. Ablation of Ikbke significantly attenuated the HDM-mediated increases in lactate (Figure $5 \mathrm{C}$ ), suggesting the requirement of lkbke in HDM-induced glycolysis. Assessment of HDM-induced airway inflammation revealed slight decreases in overall BAL cell counts in HDM-challenged $l \mathrm{kbke}^{-/-}$mice compared to WT littermates, reflected by slight decreases in neutrophils (albeit not significant), significant decreases in eosinophils, and a lack of differences in macrophages or lymphocytes (Figure 5D). Similar to our previous studies (16) significant increases airway resistance $\left(R_{N}\right)$ occurred in HDM-challenged WT mice compared to controls (Figure 5E). While HDM-exposed $\mathrm{lkbke}^{-/-}$mice showed comparable increases in baseline $\mathrm{RN}$ compared to saline-exposed mice, no further increases in $\mathrm{RN}$ in response to increasing doses of methacholine were observed. No differences in tissue resistance $(G)$ were observed between any of the groups. Converse to the attenuation of HDM-mediated increased in $\mathrm{R}_{\mathrm{N}}$ observed in HDM-exposed $/ \mathrm{kbke}^{-/-}$mice, tissue elastance was significantly elevated (Figure $5 E$ ), suggesting complex modulation of AHR in mice lacking lkbke. In WT mice, HDM led to mucus metaplasia and increases in Muc5AC in BAL (Figure 5F-G), in association with increases in IL-33 and IL-13 in lung tissues (Figure $5 \mathrm{H}$ ), consistent with a type 2, eosinophil-associated inflammatory response. In contrast, HDM-mediated increases in mucus metaplasia, Muc5AC, IL-33, IL-13, and $\mathrm{CCL}-20$ were strongly attenuated in $i k b k e^{-/-}$mice (Figure $5 \mathrm{~F}-\mathrm{H}$ ), suggesting that absence of $i k b k e$ attenuates type 2 inflammation. Although levels of TSLP were constitutively lower in $i k b k e^{-/-}$mice, compared to WT counterparts, no effect of HDM was observed at this time point (Figure $5 \mathrm{H}$ ). No differences between HDM-mediated increases in IL-1 $\beta$ were observed between WT or ikbke ${ }^{-/}$ mice (Figure $5 \mathrm{H}$ ), suggesting that IL-1 $\beta$ is increased proximally to, or independently of, ikbke. Because of these findings, the attenuation of HDM-induced lactate in lung tissues from ikbke ${ }^{-/-}$ mice, compared to WT littermates (Figure $5 \mathrm{C}$ ), and the previously reported role of IKKE in glycolytic reprogramming of DCs (21), we next addressed the role of $i k b k e$ in IL-1 $\beta$-mediated increases in glycolysis in lung tissue. IL1 $\beta$ administration was sufficient to increase lactate in WT mice. The IL-1 $\beta$-mediated increases in lactate were almost completely abolished in $i k b k e^{-/-}$mice (Figure $5 \mathrm{I}$ ), suggesting that $i k b k e$ is required for IL-1 $\beta$-induced glycolysis. 
A

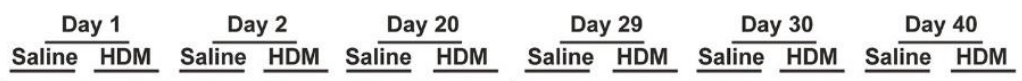

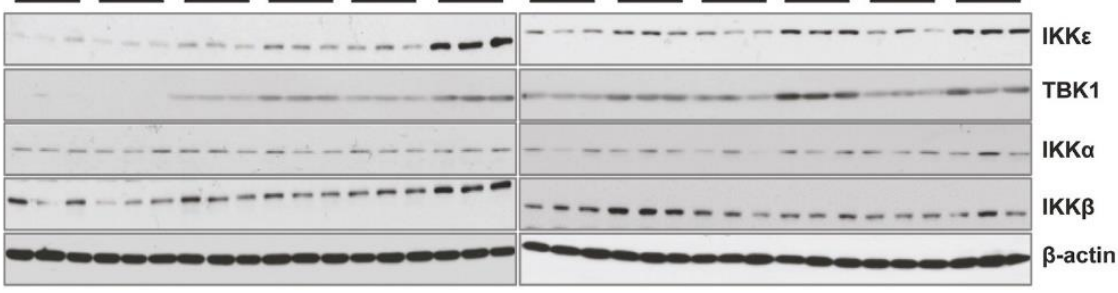

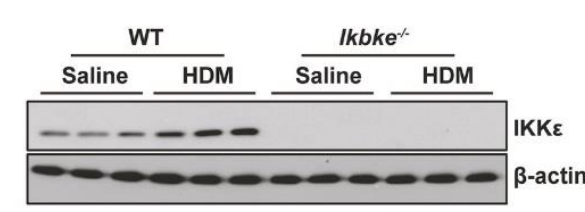

C

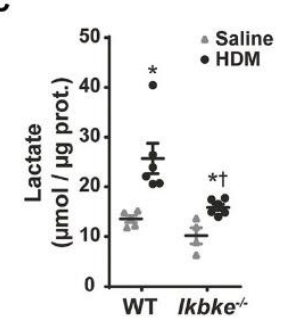

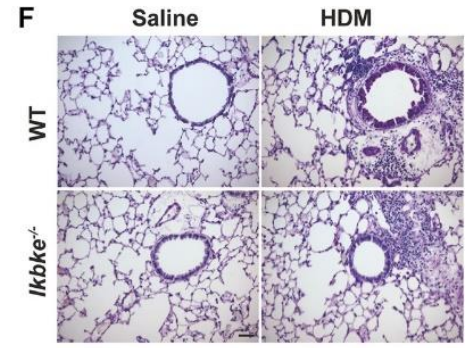

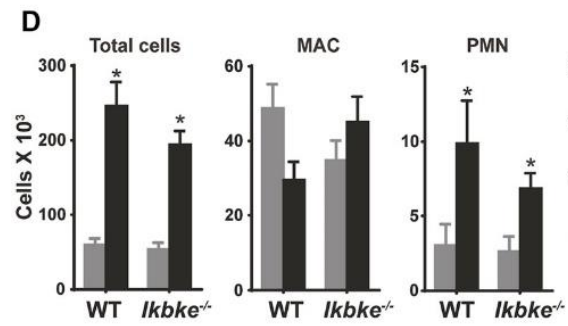

$\mathrm{E}$
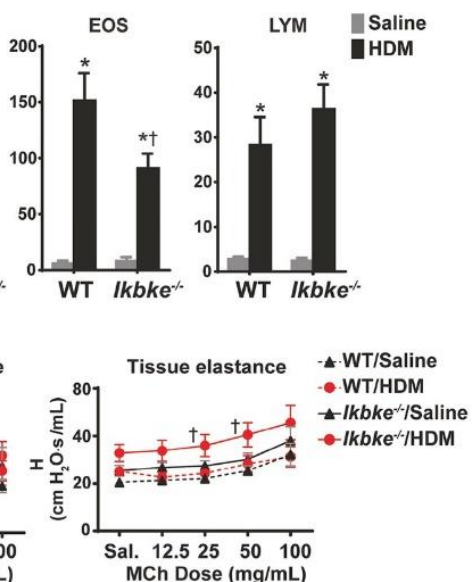

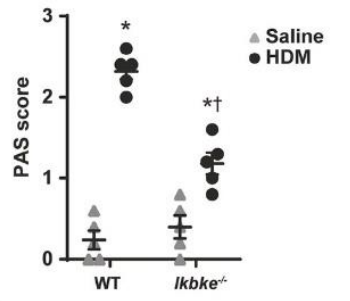

G

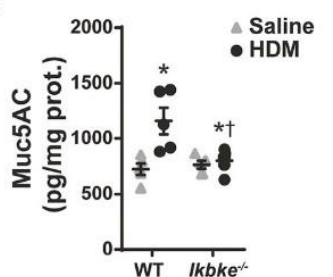

H

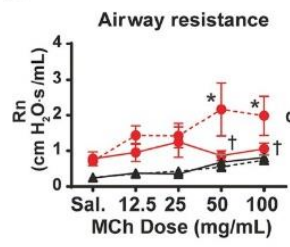

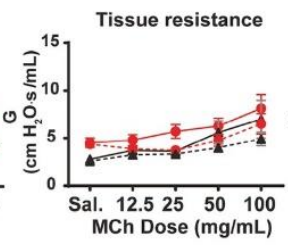

I
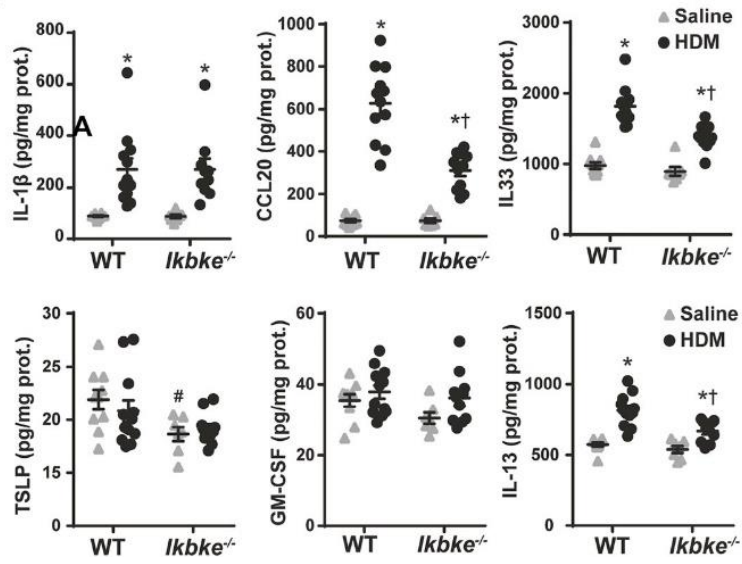

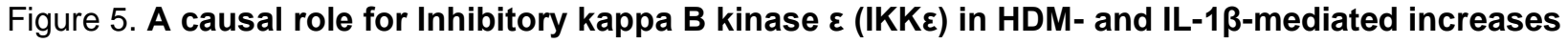
in glycolysis and the pathogenesis of allergic airways diseases. A, Western blot analyses of IKKs in lung tissues of WT mice subjected to the HDM regimen for the indicated times. WT or lkbke -/- mice were exposed as described in Fig. 1. Mice were euthanized at day 20 for assessment of IKKE in lung tissue via 
Western blot analysis (B), lactate levels in lung tissues (C), total and differential cell counts in BAL fluid (D), and AHR (E). ${ }^{*} P<0.05$ compared to the saline control group, $\dagger P<0.05$ compared to respective wildtype (WT) (ANOVA, $\mathrm{n}=5-10$ per group). F, Assessment of mucus metaplasia in WT or $/ \mathrm{kbke}^{-/-}$mice exposed to HDM or saline (scale bar, $50 \mu \mathrm{m}$ ) (Top). Quantification of airway mucus staining (PAS) intensity (Bottom). Data are expressed as means ( \pm SEM) from five mice per group. ${ }^{*} P<0.05$ compared with respective saline controls. $\mathrm{P}<0.05$ compared with WT HDM groups (Kruskal-Wallis). Levels of Muc5AC (G) and pro-inflammatory mediators $(\mathbf{H})$ in lung tissues of WT and $l k_{b k e^{-/}}$mice exposed to HDM as described in B-E. I, BAL and lung lactate levels in WT and $I k b k e^{-/-}$mice exposed to IL-1 $\beta$ for $24 \mathrm{~h}$. ${ }^{*} P<$ 0.05 relative to $V e h$ (vehicle) control group, $\dagger P<0.05$ relative to the respective wild-type (WT) group (ANOVA, $n=5-10$ per group).

Strong increases in $\mathrm{IKK} \varepsilon$ immunolocalization were observed in bronchial epithelial cells in

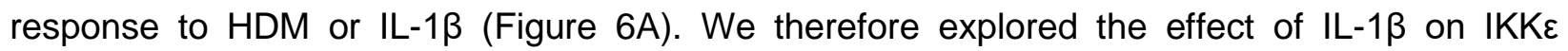

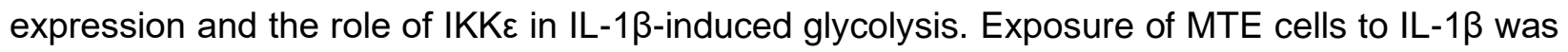
sufficient to upregulate ikbke mRNA (Figure 6B). IL-1ß-mediated increases in lactate were attenuated in $\mathrm{ikbke}^{-/-}$MTE cells (Figure 6C). Similarly, the IKKE/TBK1 inhibitor, Amlexanox resulted in a dose-dependent decrease in IL1 $\beta$-induced lactate in MTE cells (Figure 6D), and abrogated IL1ß-mediated increases in Glut1, Hk2, Ldha, and Pkm2 mRNA (Figure 6E). The more potent effects of Amlexanox compared to ikbke ablation are potentially due to Amlexamox

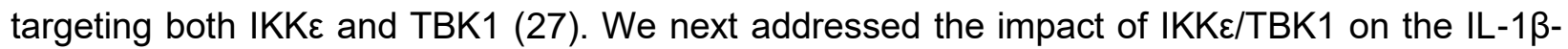
mediated augmentation of HDM-induced pro-inflammatory responses. WT cells were treated with Amlexamox overnight, followed by treatment with IL-1 $\beta$ for $24 \mathrm{~h}$. Cells were then washed and exposed to HDM for $2 \mathrm{~h}$ (Figure 6F). Similar to results in Figure 4E, prior exposure to IL-1 $\beta$ led to an augmentation of HDM-induced release of pro-inflammatory mediators from MTE cells (Figure 6F). Amlexanox ablated the IL-1 $\beta$ plus HDM-mediated increases in TSLP and GM-CSF, and attenuated CCL20 and KC (Figure 6F), identical to our findings with 2-DG (Figure 4E). Comparative evaluation of WT and Ikbke-/- epithelial cells demonstrated a strong attenuation of IL-1ß/HDM-mediated increases of TSLP, and a modest attenuation of KC and GM-CSF, while CCL20 was increased equally in Ikbke-/- cells and WT cells in response to IL-1ß/HDM. (Figure $6 \mathrm{G})$. Overall, these data suggest that IKKE is a critical mediator in IL-1 $\beta$-induced glycolysis and subsequent augmentation of HDM-mediated increases of TSLP in airway epithelial cells, and that the further decreases in CCL20, GM-CSF and KC observed in response to Amlexamox in these settings (Figure 6F) may be attributable to TBK1. 
A

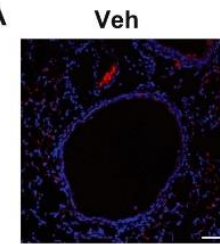

IL-1 $\beta$
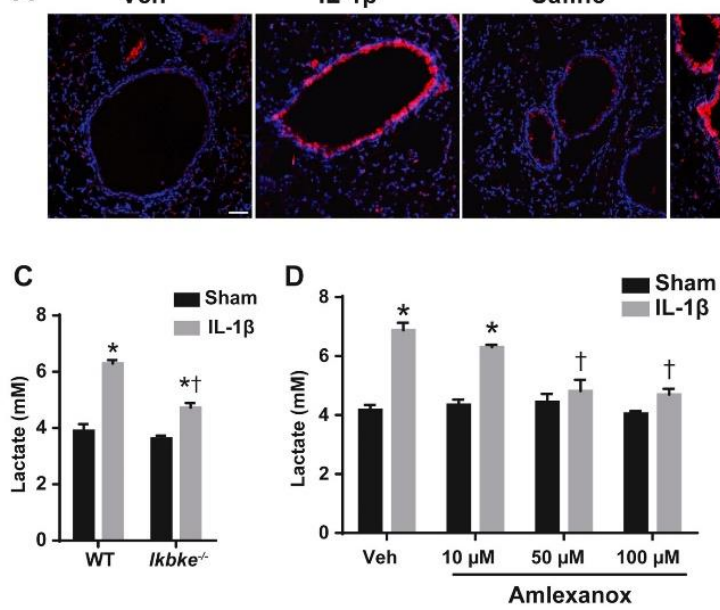

HDM

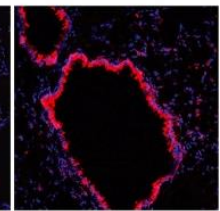

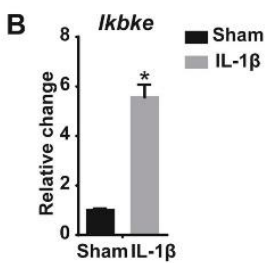

E

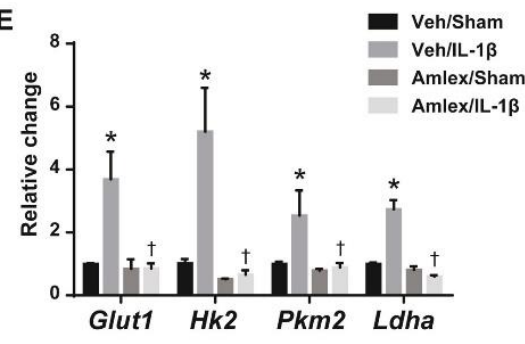

$\mathbf{F}$

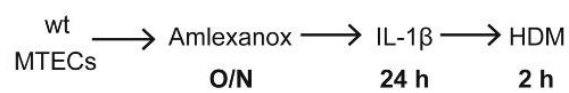

G

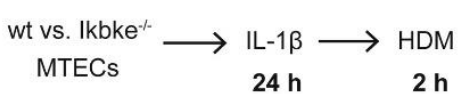
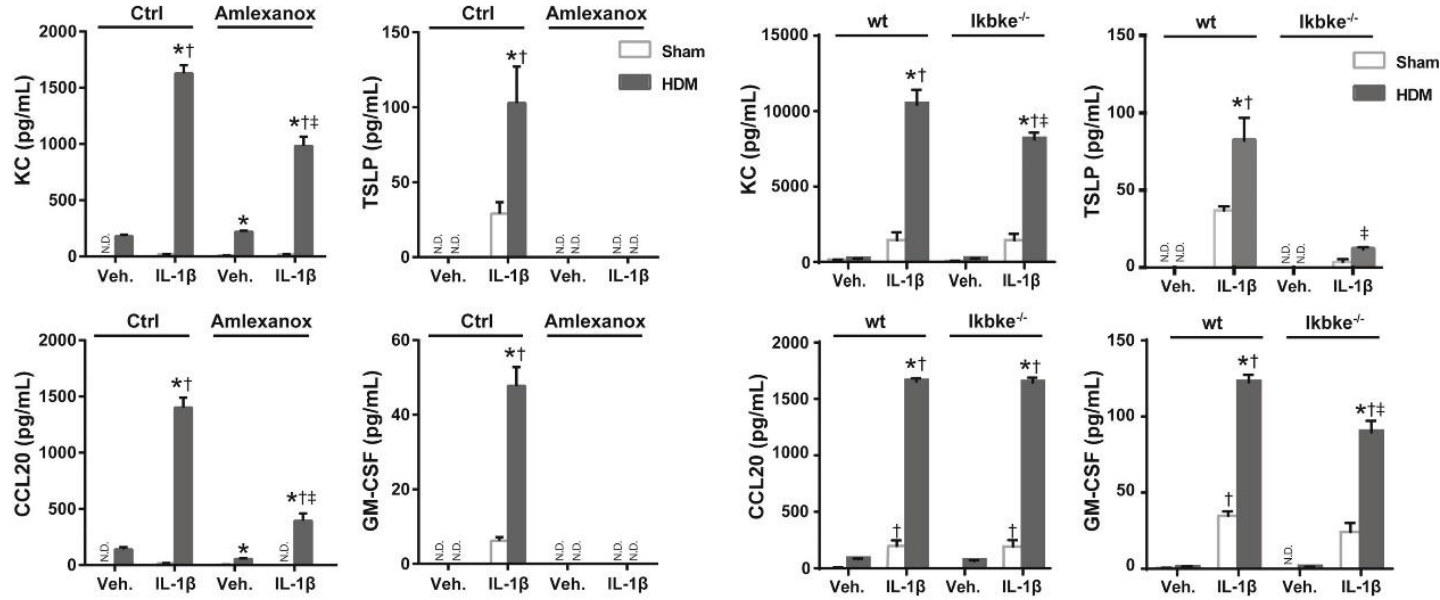
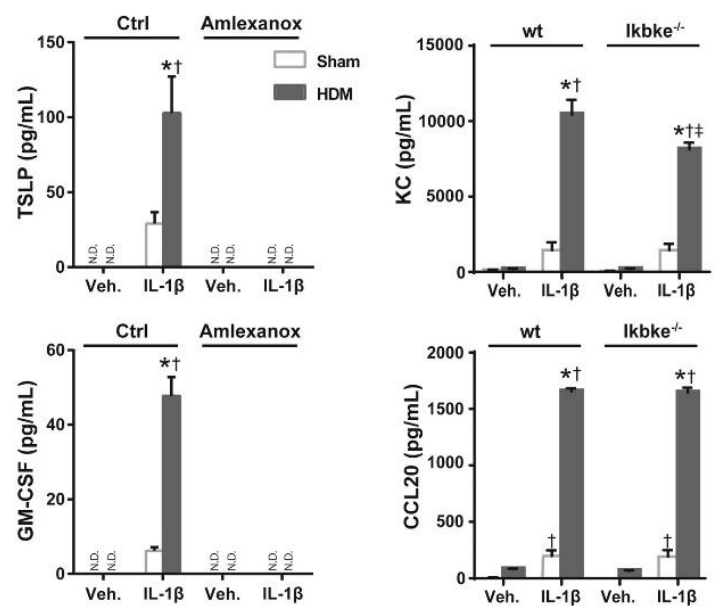

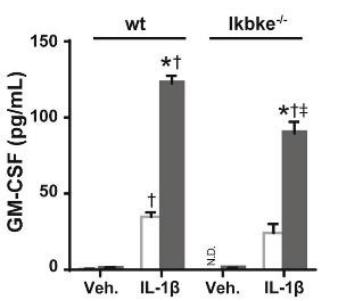

Figure 6. Inhibitory kappa B kinase $\varepsilon(\mathrm{IKK} \varepsilon)$ is required for IL-1 $\beta$-mediated increases in glycolysis, and the IL-1 $\beta$-mediated augmentation of HDM-induced innate cytokine responses in MTE cells. A,

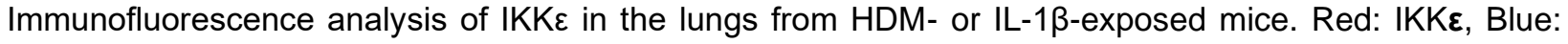
DAPI counterstain (scale bar, $50 \mu \mathrm{m}$ ). B, mRNA expression of Ikbke in MTE cells exposed to IL-1 $1 \beta$. $P<$ 0.05 relative to sham control (Student's $t$ test). $\mathbf{C}$, Lactate levels in supernatants of WT or Ikbke ${ }^{-/-}$MTE cells stimulated with IL-1 $\beta$ for $24 \mathrm{~h}$. ${ }^{*} P<0.05$ compared to sham controls, $\uparrow P<0.05$ relative to respective WT (ANOVA). D, Lactate levels in cell culture supernatants of MTE cells treated with vehicle or amlexanox, at the indicated concentrations. ${ }^{*} P<0.05$ compared to sham controls (Student's $t$ test). E, Attenuation of IL$1 \beta$-induced expression of glycolysis genes in MTE cells pre-treated with $100 \mu \mathrm{M}$ amlexanox. ${ }^{*} P<0.05$ relative to the veh/sham group, $\uparrow P<05$ relative to Veh/IL-1 $\beta$ (ANOVA). F MTE cells were pre-treated with $100 \mu \mathrm{M}$ amlexanox, followed by stimulation of IL-1 $\beta$ for $24 \mathrm{~h}$ prior to exposure to HDM (50 $\mu \mathrm{g} / \mathrm{ml}) \mathrm{for}$ an additional $2 \mathrm{~h}$ according to the indicated schematic. KC, CCL20, TSLP and GM-CSF in the cell culture supernatants of mouse tracheal epithelial cells. G KC, CCL20, TSLP and GM- CSF levels in supernatants 
of WT or Ikbke-l- MTE cells sequentially exposed to IL-1 $\beta$ and HDM according to the schematic. ${ }^{*} P<0.05$ relative to non-HDM exposed sham group, $\dagger P<0.05$ compared to respective non-IL-1 $\beta$ treated vehicle group (Veh.), and $\ddagger P<0.05$ relative to respective non-amlexanox treated control group (Figure $\mathrm{F}$ ) or wt group (Figure G) (two-way ANOVA).

\section{Lactate dehydrogenase A (LDHA) augments lactate levels in lung tissues and contributes to HDM-induced allergic airways disease}

To address the functional importance of increased glycolysis, we administered Ldha siRNA in mice with pre-existing allergic airways disease (Figure 7A). SiRNA-mediated ablation of Ldha attenuated HDM-mediated increases in LDHA expression (Figure 7B) and lactate (Figure 7C), and markedly decreased HDM-mediated increases in airway inflammation (Figure 7D). Ldha siRNA attenuated tissue levels of IL-33, IL-13 and CCL-20, but did not affect GM-CSF, IL-1 $\beta$, or TSLP (Figure 7E). Ldha siRNA decreased HDM-induced mucus metaplasia and diminished Muc5AC levels in BAL in HDM-exposed mice (Figure 7F-G), consistent with diminished type 2 inflammatory responses. Although siRNA-mediated ablation of Ldha did not affect $\mathrm{Rn}$, it attenuated tissue resistance and elastance, compared to Ctrl siRNA HDM-exposed mice (Figure 7H). Collectively, these findings point to the functional relevance of LDHA-linked glycolysis in HDM-induced airways disease, and that increases in glycolysis are an important pro-inflammatory signal.

\section{Evidence for increased glycolysis in human asthma in association with airway neutrophils}

In order to address the relevance of these findings for human asthma, we evaluated increases in glycolysis proteins and lactate in primary nasal epithelial cells (NECs). Protein levels of LDHA and pyruvate kinase M2 (PKM2) were constitutively increased in NECs from asthmatics as compared to controls (Figure 8A, Figure E5A), in association with increases in lactate (Figure 8B). In response to HDM, no further differences in expression of these mediators were observed. These findings suggest that asthmatic NECs show an intrinsic increase in glycolysis. Assessment of cell-free sputum samples of healthy subjects $(n=20)$ or asthmatics $(n=94)$ showed increased lactate levels in asthmatics as compared to controls (Figure $8 \mathrm{C}$ ). Sputum lactate levels negatively correlated with \%FEV1 in asthmatics but not in healthy individuals (Figure 8D). Although overall levels of IL-1 $\beta$ in sputum samples were not significantly higher in the overall asthmatic population, than those in controls (Figure 8E) a significant correlation was apparent between lactate and IL$1 \beta$ in asthmatics (Figure 8F). Given the large fluctuations in levels of lactate (range 5.3-362.9 
$\mu \mathrm{M}$ ) and IL-1 $($ range $0.8-262.8 \mu \mathrm{g} / \mathrm{ml}$ ) in the asthmatic subjects we further investigated whether these parameters were related to specific clinical features. Lactate or IL-1 $\beta$ were not elevated in patients with eosinophilic asthma ( $>3 \%$ sputum eosinophils) compared to patients with low eosinophils ( $<3 \%$ eosinophils, $p=0.81$ and 0.57 , respectively). Lactate was not different between atopic and non-atopic asthmatics $(p=0.67)$. IL-1 $\beta$ levels trended towards being elevated in atopic compared to non-atopic asthmatics $(p=0.07)$. Lactate and IL-1 $\beta$ values trended towards increases in asthmatic patients who received corticosteroids compared to the patients who did not $(p=0.09$ and 0.07 , respectively). Lactate and IL-1 $\beta$ were significantly elevated in neutrophilic asthmatics ( $>61 \%$ sputum neutrophils, Figure E5B) compared to patients with $<61 \%$ neutrophils. Lactate levels (but not IL-1 $\beta$ ) were significantly higher in patients whose asthma was uncontrolled (Figure E5B). The BMI was increased in asthmatics as compared to healthy subjects (Table E1). Adjustment for BMI still showed significant increases in lactate in asthmatics as compared to healthy subjects $(p=0.0002)$. Collectively, these data suggest that IL-1-linked glycolysis is an important feature of allergic asthma. 
A

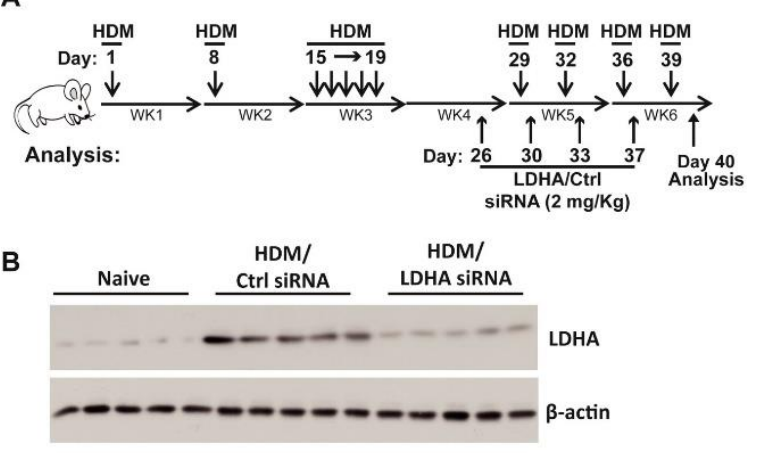

E
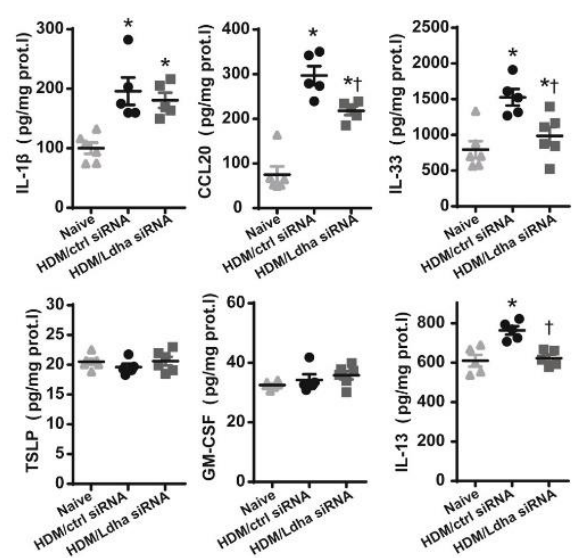

C
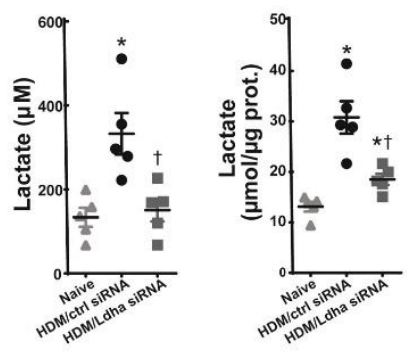

D

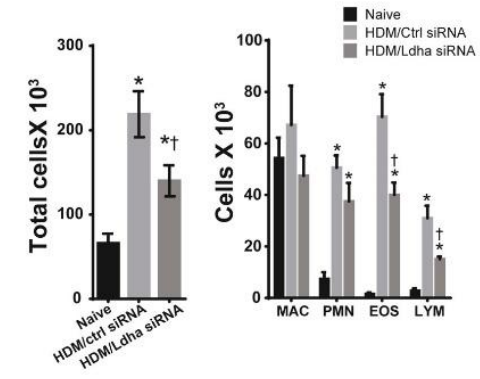

G

H
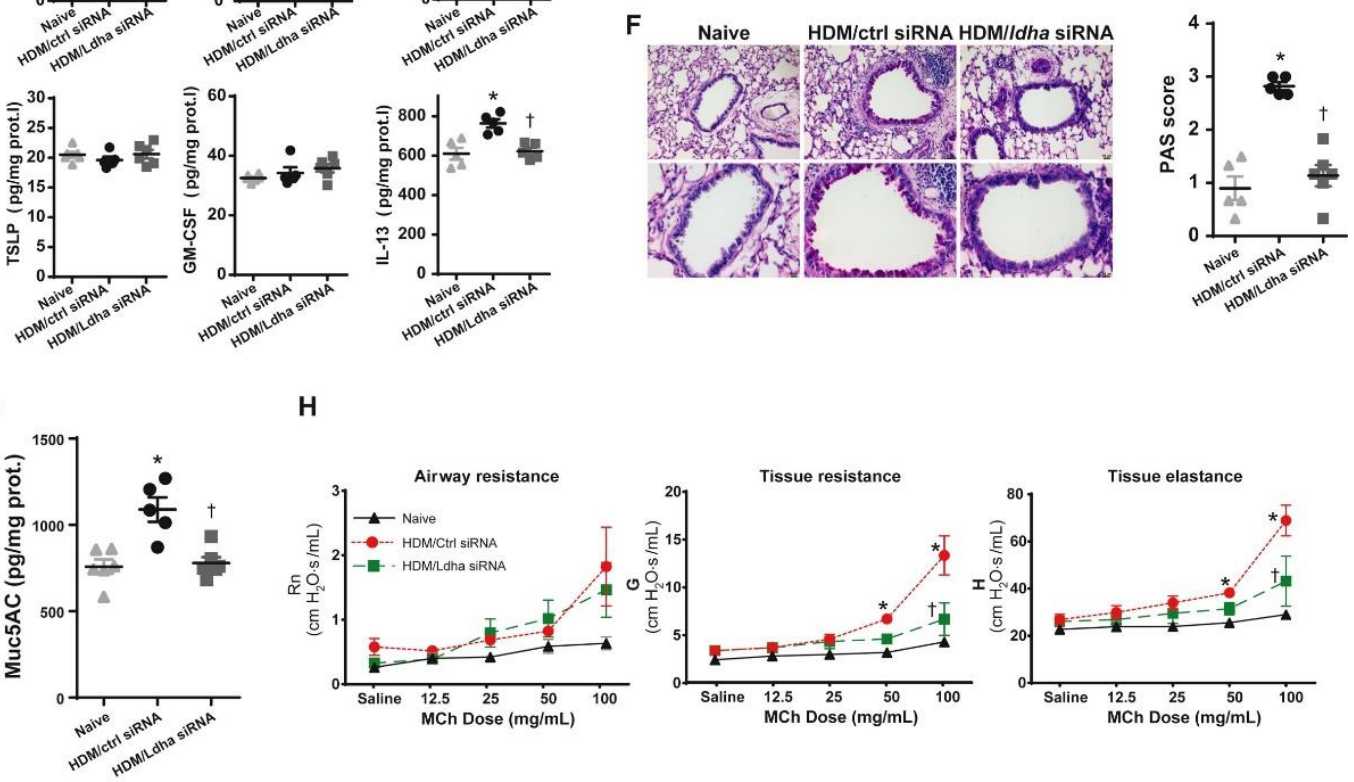

Figure 7. siRNA-mediated knockdown of Ldha attenuates HDM-mediated increase in glycolysis, airway inflammation, and airways hyperresponsiveness. A, Schematic depicting the dosing regimen of HDM, control (Ctrl) and Ldha siRNAs. At day 40, Salineexposed mice or HDM-exposed mice treated with Ctrl siRNA or Ldha siRNA were harvested for the assessment of LDHA protein levels in the lung tissues via Western blot analyses (B) levels of lactate in BAL and lung tissue $(\mathbf{C})$, total and differential cell counts in the BAL (D), levels of IL-1 $\beta$, CCL20, IL-33, TSLP, GM-CSF, and IL-13 in the lung tissue (E). ${ }^{*}<0.05$ relative to the naive group, $\dagger P<0.05$ relative to the HDM/Ctrl siRNA group (ANOVA). F, Periodic acid Schiff (PAS) staining of airway mucus in saline- or HDM-exposed mice treated with Ctrl siRNA or Ldha siRNA (scale bar, $50 \mu \mathrm{m}$ ) (Left). Quantification of airway mucus staining (PAS) intensity (Right). Data are expressed as means $\left( \pm\right.$ SEM) from five-six mice per group. ${ }^{*} P<0.05$ compared with naive mice. $\dagger P<0.05$ compared to HDM/ctrl siRNA group (Kruskal Wallis) G, Measurement of muc5AC levels in the BAL from 
mice described in A-E. $\mathbf{H}$, Assessment of AHR. ${ }^{*} P<0.05$ relative to naive group, $\dagger P<0.05$ relative to HDM/Ctrl siRNA group (ANOVA).

A

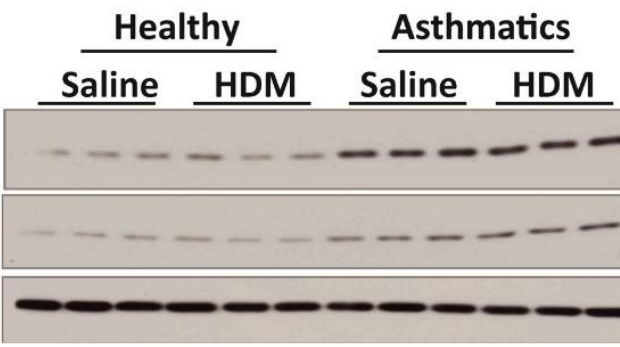

B

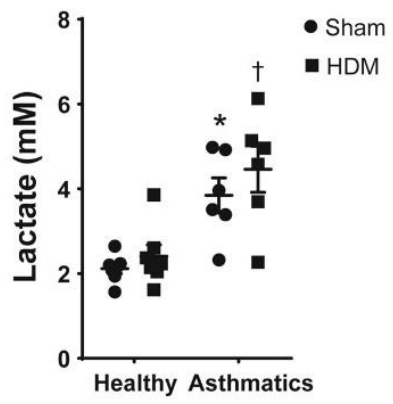

C

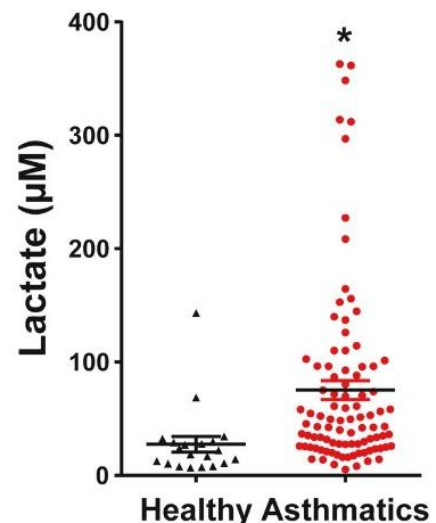

E

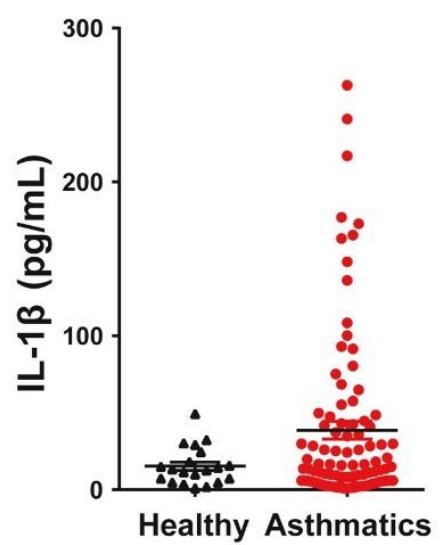

D

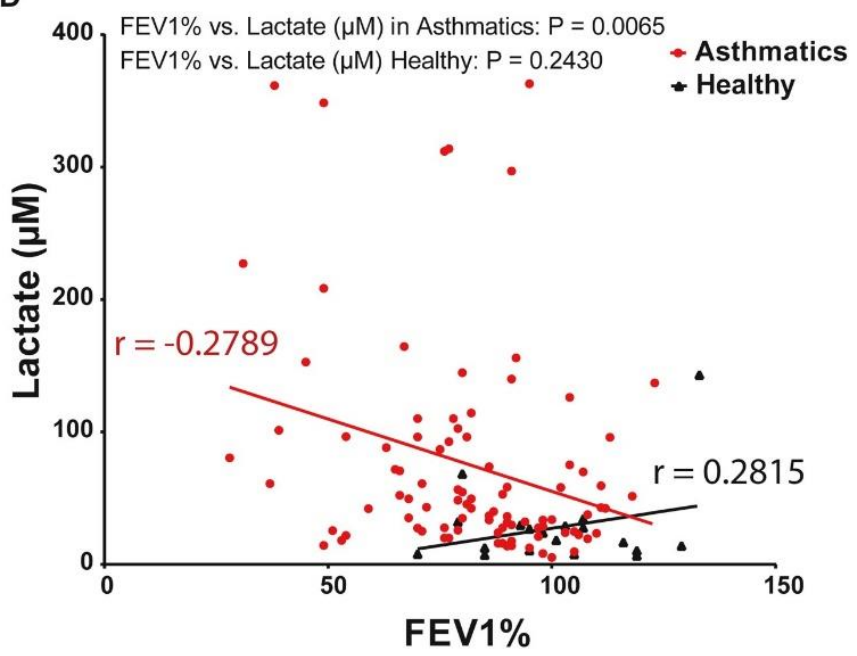

$\mathbf{F}$

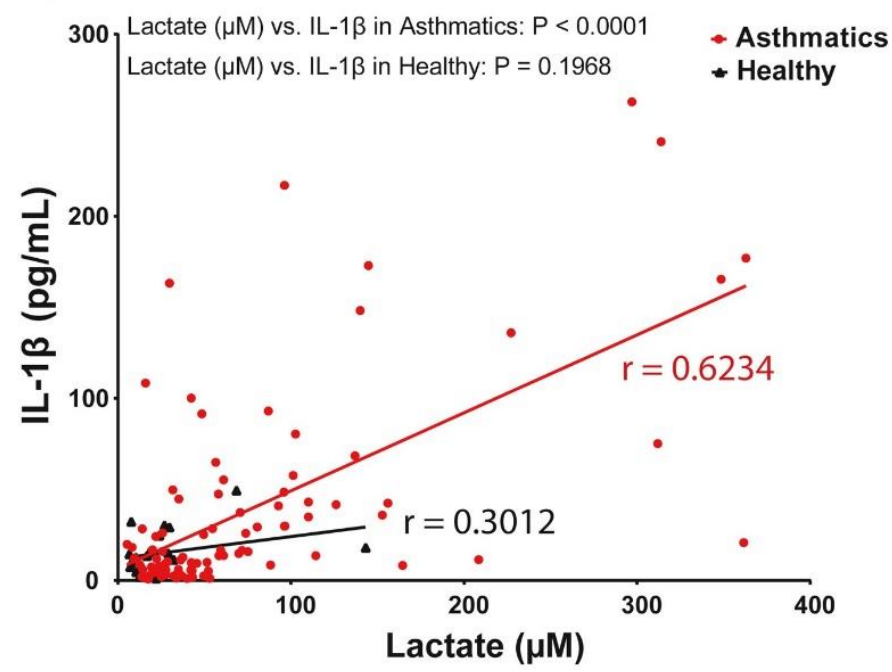

Figure 8. Evidence of increases in glycolysis in human asthma. A, Western blot analysis of PKM2 and LDHA, in saline or HDM-treated nasal cells isolated from asthmatics or healthy individuals. Data are representative of 6 healthy subjects, and 6 asthmatics $\mathbf{B}$, Lactate content in culture supernatants of cells shown in $\mathrm{A}$. ${ }^{*} P<0.05$ compared to cells from healthy controls not exposed to HDM, $\uparrow P<0.05$ compared 
to cells from healthy controls exposed to HDM, (ANOVA). C-F, Lactate (C) and IL-1 $\beta$ levels (E) in the sputum supernatants from healthy subjects $(n=20)$ or asthmatics $(n=94)$. Correlations between lactate content and forced expiratory volume in $1 \mathrm{~s}$ percentage predicted (FEV1\%) (D) or IL-1 $\beta$ levels (F) in asthmatics and healthy subjects. Correlation analyses were performed via Spearman rank correlation coefficients.

\section{Discussion}

Perturbations in glycolysis are implicated in the pathogenesis of several chronic inflammatory diseases $(10,28)$. However, the role of dysregulated glycolysis in allergic asthma is not well appreciated. Herein, we discovered that in mice with HDM-induced allergic airways disease, glycolysis was increased in association with HDM-induced inflammation, mucus metaplasia, and AHR. Our results also illuminated that IL-1- and IKKE-dependent signals are important in augmenting glycolysis in HDM-exposed mice, and in enhancing HDM-induced pro-inflammatory signals in epithelial cells. Importantly, inhibition of glycolysis via administering Ldha siRNA in mice with pre-existing allergic airways disease attenuated the patho-physiological manifestations of allergic airways disease. These findings have potential relevance to human asthma given the robust increases in expression of LDHA and increased levels of lactate in primary human NECs and cell culture supernatants, respectively, and the observed positive correlation between lactate and IL-1 $\beta$ in asthmatic sputum samples.

In the present study we demonstrated the importance of IL-1 signaling in mediating HDM-induced glycolysis. This claim is based upon findings demonstrating that increases in IL-1 $\beta$ levels were temporally correlated with increases in lactate in response to HDM, that neutralization of IL-1 significantly attenuated HDM-induced glycolysis, and that administration of IL-1 $\beta$ into airways or to MTE cells was sufficient to increase glycolysis. These findings are consistent with earlier studies showing that IL-1 signaling increases glycolysis during Th17 cell differentiation (29), and in mesangial cells (30). IL-1 $\alpha$ and IL-1 $\beta$ share biological activity by acting exclusively on Interleukin 1 receptor, type I (IL1RI) (31), and various studies suggest that both cytokines play critical roles in asthma (32-37). Neutralizing IL-1 $\alpha$ during allergic sensitization to HDM resulted in strongly attenuated Th2 inflammation (36). Although we did not detect increases in IL-1 $\alpha$ at times that corresponded with increases in lactate, we cannot rule out the possibility that IL-1 $\alpha$ may be involved in increased glycolysis in settings of allergic airways disease. As IL-1 $\beta$ and IL-1 $\alpha$ both activate IL-1RI, and increase glycolysis in epithelial cells (Figure 3) and IL1-Trap lowered both IL$1 \beta$ and $\mathrm{IL}-1 \alpha$ in lung tissue (Figure E2), their relative contributions in mediating HDM-induced 
glycolysis need to be further dissected. Lastly, we also report that HDM-induced adaptive immunity is required for the observed increases in IL-1 $\beta$ and associated increases in lactate.

Alterations in cellular metabolism are known to affect function of immune cells (9), and increases in glycolysis have been shown to regulate immune effector function (21, 22, 38-41). Despite these studies, the role of enhanced glycolysis in structural cells such as airway epithelium and implications for their innate effector function has remained unknown. Here, we demonstrate that inhibition of glycolysis via targeting HK or LDHA markedly dampened IL-1 $\alpha$ - or IL-1 $\beta$-induced proinflammatory responses, and strongly attenuated the ability of IL-1 $\beta$ to augment HDM-induced innate cytokine responses in MTE cells. Overall these findings suggest that enhanced glycolysis is important for the amplification of allergen-induced pro-inflammatory responses. However, further mechanistic studies will be required to unravel how glycolysis modulates pro-inflammatory responses in epithelial cells. Rapid ATP generation during glycolysis is required for immediate energy demand during immune cell proliferation and activation (9). We and others have shown that extracellular ATP activates purinergic receptors, leading to release of IL-33 from epithelial cells (42), suggesting a potential mechanism whereby increased glycolysis augments epithelial effector function.

In the present study we also demonstrate that IKKE expression is increased in bronchial epithelium in response to HDM or IL-1 3 , and that it promotes glycolysis and pro-inflammatory responses in epithelial cells and contributes to HDM-induced allergic airways disease. However, the molecular details whereby IL-1 and IKKE enhance glycolysis remain unknown. IL-1 has recently been shown to activate IKte and subsequent AKT-mTOR signaling pathway, leading to Th17 cell maintenance (25); and, AKT or mTOR, when activated, are known to induce glycolysis $(21,43)$, suggesting the potential role of AKT-mTOR signaling in HDM/IL-1/IKKE-induced glycolysis.

The connection of IL-1 signaling and glycolysis described herein in the murine model of allergic airway disease is corroborated by our findings in samples from asthmatics. As was mentioned above, lactate was significantly higher in sputum samples of asthmatics. Furthermore, lactate and IL-1 $\beta$ level and were positively correlated in asthmatic sputum supernatants, and lactate negatively correlated with lung function. Lactate and IL-1 $\beta$ were notably increased in patients with neutrophilic asthma ( $\geq 61 \%$ neutrophils), whereas no correlations between these parameters and eosinophils were observed. IL-1 $\beta$ has implicated in a number of pulmonary diseases (44-46). Although increases in IL-1 $1 \beta$ observed herein are not specific to only patients with asthma, IL-1 $1 \beta$ is emerging as a key cytokine relevant to the pathogenesis of asthma (47). IL-1 $\beta$ has been linked 
to severe, neutrophilic, steroid insensitive asthma in a mouse model (48). In contrast to the present data, a recent study suggested a critical role for the IL-1 $\beta$ pathway in patients with $\mathrm{T}_{\mathrm{H}} 2 / \mathrm{T}_{\mathrm{H}}$ 17-predominant asthma (having $4 \%$ of $\mathrm{BAL}$ neutrophils) whereas IL-1a was linked to neutrophilic asthma (having 16\% BAL neutrophils) (49). The discrepancy between these findings may be associated with differences in patient characteristics, sampling (sputum as compared to BAL analyses) and illuminates the complexities among the various asthma subtypes. Therefore, additional studies will be essential to unravel the contributions of IL-1 $\alpha, \mathrm{IL}-1 \beta$ and activation of glycolysis pathways in the asthma subtypes. Excessive $\beta$-agonist administration has been associated with elevated plasma lactate levels (50-52). We believe it is unlikely that salbutamol used to induce sputum in our study contributed to the increased level of sputum lactate because a low dose of salbutamol $(400 \mu \mathrm{g})$ was used, and both healthy and asthmatic patients received salbutamol. This notion is also backed by our findings that asthmatic NECs expressed more LDHA and produced more lactate as compared to controls, in the absence of exposure to $\beta$-agonists (Figure 8). The latter findings also suggests that human NECs from asthmatics are intrinsically different from their counterparts derived from healthy individuals. Considering that IL-1 proteins can be produced by epithelial cells (36), it will be interesting to elucidate whether epithelial IL-1 and IL-1RI signaling form an autocrine loop that sustains the constitutive over-production of lactate observed in NECs derived from asthmatics. Furthermore, an epigenetic mechanism may also be involved in this process, as a recent study discovered that, during Th1 cell differentiation, LDHA-mediated increases in glycolysis maintain a high concentration of acetyl-coenzyme $A$ that in turn enhances histone acetylation (53).

In summary, the present study demonstrates the importance of glycolysis in the pathophysiology of allergic airways disease, and suggests that targeting glycolysis $(6,54,55)$ may ultimately provide a new approach in the treatment of asthma. Additional studies will be required to elucidate the cell types wherein enhanced glycolysis occurs in settings of asthma. Similarly, the molecular details whereby changes in glycolysis regulate the effector function of epithelial and other cell types also warrant further investigation.

\section{Key message:}

- Primary nasal epithelial cells from asthmatics intrinsically express more LDHA and produce more lactate as compared to healthy controls, and sputum lactate levels negatively correlate with lung function in asthmatics. 
- The IL-1/IKKE signaling axis mediates HDM-induced glycolysis and allergic airways disease in mice.

- Increases in glycolysis are critical in the augmentation of HDM-triggered proinflammatory Abbreviations:

- AHR: Airways hyperresponsiveness

- LDHA: Lactate dehydrogenase A

- HDM: House dust mite

- WT: Wild-type

- HK1: Hexokinase 1

- HK2: Hexokinase 2

- MTE cells: Mouse tracheal epithelial cells

- ECAR: Extracellular acidification rates

- OCR: Oxygen consumption rates

- 2-DG: 2-Deoxyglucose

- IKK: Inhibitory kappa B kinase

- IL1RI: Interleukin 1 receptor, type I

- TLR4: Toll like receptor-4

- TGF $\beta$ : Transforming growth factor $\beta$

- GAPDH: Glyceraldehyde 3-phosphate dehydrogenase

- IFNy: Interferon y

- NECs: Nasal epithelial cells

- PKM2: Pyruvate kinase M2 


\section{REFERENCES:}

1. Moorman JE, Akinbami $L$, Bailey C, Zahran H, King M, Johnson C, et al. National surveillance of asthma: United States, 2001-2010 Vital \& health statistics Series 3, Analytical and epidemiological studies/[US Dept of Health and Human Services, Public Health Service, National Center for Health Statistics; ]. 2012(35):1-67.

2. Gauthier M, Ray A, Wenzel SE. Evolving concepts of asthma. American journal of respiratory and critical care medicine. 2015;192(6):660-8.

3. Fahy JV. Type 2 inflammation in asthma--present in most, absent in many. Nature Reviews Immunology. 2015;15(1):57-65.

4. Erle DJ, Sheppard D. The cell biology of asthma. The Journal of cell biology. 2014;205(5):621-31.

5. Hotamisligil GS. Inflammation and metabolic disorders. Nature. 2006;444(7121):860-7.

6. Doherty JR, Cleveland JL. Targeting lactate metabolism for cancer therapeutics. The Journal of clinical investigation. 2013;123(9):3685-92.

7. Ward PS, Thompson CB. Metabolic reprogramming: a cancer hallmark even warburg did not anticipate. Cancer cell. 2012;21(3):297-308.

8. Nathan C, Ding A. Nonresolving inflammation. Cell. 2010;140(6):871-82.

9. O'Neill LA, Kishton RJ, Rathmell J. A guide to immunometabolism for immunologists. Nature Reviews Immunology. 2016.

10. Haas R, Smith J, Rocher-Ros V, Nadkarni S, Montero-Melendez T, D'Acquisto F, et al. Lactate regulates metabolic and pro-inflammatory circuits in control of $t$ cell migration and effector functions. PLoS Biol. 2015;13(7):e1002202.

11. Leppänen $O$, Björnheden T, Evaldsson M, Borén J, Wiklund O, Levin M. ATP depletion in macrophages in the core of advanced rabbit atherosclerotic plaques in vivo. Atherosclerosis. 2006;188(2):323-30.

12. Colegio OR, Chu N-Q, Szabo AL, Chu T, Rhebergen AM, Jairam V, et al. Functional polarization of tumour-associated macrophages by tumour-derived lactic acid. Nature. 2014;513(7519):559-63.

13. Ostroukhova M, Goplen N, Karim MZ, Michalec L, Guo L, Liang Q, et al. The role of low-level lactate production in airway inflammation in asthma. American Journal of Physiology-Lung Cellular and Molecular Physiology. 2012;302(3):L300-L7.

14. Rajamäki K, Nordström T, Nurmi K, Äkerman KE, Kovanen PT, Öörni K, et al. Extracellular acidosis is a novel danger signal alerting innate immunity via the NLRP3 inflammasome. Journal of Biological Chemistry. 2013;288(19):13410-9.

15. Wike-Hooley J, Haveman J, Reinhold H. The relevance of tumour $\mathrm{pH}$ to the treatment of malignant disease. Radiotherapy and Oncology. 1984;2(4):343-66.

16. Tully JE, Hoffman SM, Lahue KG, Nolin JD, Anathy V, Lundblad LK, et al. Epithelial NF-kB orchestrates house dust mite-induced airway inflammation, hyperresponsiveness, and fibrotic remodeling. The Journal of Immunology. 2013;191(12):5811-21. 
17. Doherty JR, Cleveland JL. Targeting lactate metabolism for cancer therapeutics. The Journal of clinical investigation. 2013;123(9):3685.

18. Ather JL, Foley KL, Suratt BT, Boyson JE, Poynter ME. Airway epithelial NF-KB activation promotes the ability to overcome inhalational antigen tolerance. Clinical \& Experimental Allergy. 2015;45(7):1245-58.

19. Mombaerts $P$, lacomini J, Johnson RS, Herrup K, Tonegawa S, Papaioannou VE. RAG-1-deficient mice have no mature B and Tlymphocytes. Cell. 1992;68(5):869-77.

20. Hoffman SM, Chapman DG, Lahue KG, Cahoon JM, Rattu GK, Daphtary N, et al. Protein disulfide isomerase-endoplasmic reticulum resident protein 57 regulates allergen-induced airways inflammation, fibrosis, and hyperresponsiveness. Journal of Allergy and Clinical Immunology. 2016;137(3):822-32. e7.

21. Everts B, Amiel E, Huang SC-C, Smith AM, Chang C-H, Lam WY, et al. TLR-driven early glycolytic reprogramming via the kinases TBK1-IKK [epsiv] supports the anabolic demands of dendritic cell activation. Nature immunology. 2014;15(4):323-32.

22. Krawczyk CM, Holowka T, Sun J, Blagih J, Amiel E, DeBerardinis RJ, et al. Toll-like receptor-induced changes in glycolytic metabolism regulate dendritic cell activation. Blood. 2010;115(23):4742-9.

23. Pantano C, Ather JL, Alcorn JF, Poynter ME, Brown AL, Guala AS, et al. Nuclear factor-KB activation in airway epithelium induces inflammation and hyperresponsiveness. American journal of respiratory and critical care medicine. 2008;177(9):959-69.

24. Ather JL, Hodgkins SR, Janssen-Heininger YM, Poynter ME. Airway epithelial NF-kB activation promotes allergic sensitization to an innocuous inhaled antigen. American journal of respiratory cell and molecular biology. 2011;44(5):631-8.

25. Gulen MF, Bulek K, Xiao H, Yu M, Gao J, Sun L, et al. Inactivation of the enzyme GSK3 $\alpha$ by the kinase IKKi promotes AKT-mTOR signaling pathway that mediates interleukin-1-induced Th17 cell maintenance. Immunity. 2012;37(5):800-12.

26. Tully JE, Nolin JD, Guala AS, Hoffman SM, Roberson EC, Lahue KG, et al. Cooperation between classical and alternative NF-kB pathways regulates proinflammatory responses in epithelial cells. American journal of respiratory cell and molecular biology. 2012;47(4):497-508.

27. Reilly SM, Chiang S-H, Decker SJ, Chang L, Uhm M, Larsen MJ, et al. An inhibitor of the protein kinases TBK1 and IKK-[epsiv] improves obesity-related metabolic dysfunctions in mice. Nature medicine. 2013;19(3):313-21.

28. Shirai $T$, Nazarewicz RR, Wallis $B B$, Yanes RE, Watanabe $R$, Hilhorst $M$, et al. The glycolytic enzyme PKM2 bridges metabolic and inflammatory dysfunction in coronary artery disease. The Journal of experimental medicine. 2016;213(3):337-54.

29. Park M-J, Lee SH, Lee S-H, Lee E-J, Kim E-K, Choi JY, et al. IL-1 Receptor Blockade Alleviates Graft-versusHost Disease through Downregulation of an Interleukin-1-Dependent Glycolytic Pathway in Th17 Cells. Mediators of inflammation. 2015;2015.

30. Taneja N, Coy PE, Lee I, Bryson JM, Robey RB. Proinflammatory interleukin-1 cytokines increase mesangial cell hexokinase activity and hexokinase II isoform abundance. American Journal of PhysiologyCell Physiology. 2004;287(2):C548-C57. 
31. Garlanda C, Dinarello CA, Mantovani A. The interleukin-1 family: back to the future. Immunity. 2013;39(6):1003-18.

32. Broide DH, Campbell $K$, Gifford T, Sriramarao P. Inhibition of eosinophilic inflammation in allergenchallenged, IL-1 receptor type 1-deficient mice is associated with reduced eosinophil rolling and adhesion on vascular endothelium. Blood. 2000;95(1):263-9.

33. Schmitz $N$, Kurrer $M$, Kopf $M$. The IL-1 receptor 1 is critical for Th2 cell type airway immune responses in a mild but not in a more severe asthma model. European journal of immunology. 2003;33(4):991-1000.

34. Nakae S, Komiyama Y, Yokoyama H, Nambu A, Umeda $M$, Iwase $M$, et al. IL - 1 is required for allergen - specific Th2 cell activation and the development of airway hypersensitivity response. International immunology. 2003;15(4):483-90.

35. Whelan R, Kim C, Chen M, Leiter J, Grunstein M, Hakonarson H. Role and regulation of interleukin-1 molecules in pro-asthmatic sensitised airway smooth muscle. European Respiratory Journal. 2004;24(4):559-67.

36. Willart MA, Deswarte $K$, Pouliot $P$, Braun $H$, Beyaert $R$, Lambrecht $B N$, et al. Interleukin-1a controls allergic sensitization to inhaled house dust mite via the epithelial release of GM-CSF and IL-33. The Journal of experimental medicine. 2012;209(8):1505-17.

37. Johnson VJ, Yucesoy B, Luster MI. Prevention of IL-1 signaling attenuates airway hyperresponsiveness and inflammation in a murine model of toluene diisocyanate-induced asthma. Journal of allergy and clinical immunology. 2005;116(4):851-8.

38. Jantsch J, Chakravortty D, Turza N, Prechtel AT, Buchholz B, Gerlach RG, et al. Hypoxia and hypoxiainducible factor-1 $\alpha$ modulate lipopolysaccharide-induced dendritic cell activation and function. The Journal of Immunology. 2008;180(7):4697-705.

39. Donnelly RP, Loftus RM, Keating SE, Liou KT, Biron CA, Gardiner CM, et al. mTORC1- dependent metabolic reprogramming is a prerequisite for NK cell effector function. The Journal of Immunology. 2014;193(9):4477-84.

40. Michalek RD, Gerriets VA, Jacobs SR, Macintyre AN, Maclver NJ, Mason EF, et al. Cutting edge: distinct glycolytic and lipid oxidative metabolic programs are essential for effector and regulatory CD4+ $T$ cell subsets. The Journal of Immunology. 2011;186(6):3299-303.

41. Doughty CA, Bleiman BF, Wagner DJ, Dufort FJ, Mataraza JM, Roberts MF, et al. Antigen receptormediated changes in glucose metabolism in $B$ lymphocytes: role of phosphatidylinositol 3- kinase signaling in the glycolytic control of growth. Blood. 2006;107(11):4458-65.

42. Kouzaki H, lijima K, Kobayashi T, O'Grady SM, Kita H. The danger signal, extracellular ATP, is a sensor for an airborne allergen and triggers $I L-33$ release and innate Th2-type responses. The Journal of Immunology. 2011;186(7):4375-87.

43. Moon J-S, Hisata S, Park M-A, DeNicola GM, Ryter SW, Nakahira K, et al. mTORC1-Induced HK1Dependent glycolysis regulates NLRP3 inflammasome activation. Cell reports. 2015;12(1):102-15.

44. Pugin J, Ricou B, Steinberg KP, Suter PM, Martin TR. Proinflammatory activity in bronchoalveolar lavage fluids from patients with ARDS, a prominent role for interleukin-1. Am J Respir Crit Care Med. 1996;153(6 Pt 1):1850-6. 
45. Pauwels NS, Bracke KR, Dupont LL, Van Pottelberge GR, Provoost S, Vanden Berghe T, et al. Role of IL1alpha and the NIrp3/caspase-1/IL-1beta axis in cigarette smoke-induced pulmonary inflammation and COPD. The European respiratory journal. 2011;38(5):1019-28.

46. Rogliani P, Calzetta L, Ora J, Matera MG. Canakinumab for the treatment of chronic obstructive pulmonary disease. Pulmonary pharmacology \& therapeutics. 2015;31:15-27.

47. Peebles RS Jr. Is IL-1beta inhibition the next therapeutic target in asthma? The Journal of allergy and clinical immunology. 2017.

48. Kim RY, Pinkerton JW, Essilfie AT, Robertson AA, Baines KJ, Brown AC, et al. Role for NLRP3 Inflammasome-mediated, IL-1beta-dependent Responses in Severe, Steroid-resistant Asthma. Am J Respir Crit Care Med. 2017.

49. Liu W, Liu S, Verma M, Zafar I, Good JT, Rollins D, et al. Mechanism of TH2/TH17-predominant and neutrophilic TH2/TH17-low subtypes of asthma. The Journal of allergy and clinical immunology. 2017;139(5):1548-58 e4.

50. Rodrigo G, Rodrigo C. Elevated plasma lactate level associated with high dose inhaled albuterol therapy in acute severe asthma. Emergency Medicine Journal. 2005;22(6):404-8.

51. Lewis LM, Ferguson I, House SL, Aubuchon K, Schneider J, Johnson K, et al. Albuterol administration is commonly associated with increases in serum lactate in patients with asthma treated for acute exacerbation of asthma. CHEST Journal. 2014;145(1):53-9.

52. Dodda V, Spiro P. Albuterol, an uncommonly recognized culprit in lactic acidosis. CHEST Journal. 2011;140(4_MeetingAbstracts):183A-A.

53. Peng M, Yin N, Chhangawala S, Xu K, Leslie CS, Li MO. Aerobic glycolysis promotes Thelper 1 cell differentiation through an epigenetic mechanism. Science. 2016;354(6311):481-4.

54. Baggstrom MQ, Qi Y, Koczywas M, Argiris A, Johnson EA, Millward MJ, et al. A phase II study of AT101 (Gossypol) in chemotherapy-sensitive recurrent extensive-stage small cell lung cancer. Journal of Thoracic Oncology. 2011;6(10):1757-60.

55. Polanski R, Hodgkinson CL, Fusi A, Nonaka D, Priest L, Kelly P, et al. Activity of the monocarboxylate transporter 1 inhibitor AZD3965 in small cell lung cancer. Clinical Cancer Research. 2014;20(4):926-37. 


\section{SUPPLEMENTAL MATERIAL AND METHODS}

\section{Sputum induction}

Sputum was induced and processed, as described previously (1-3). Prior to sputum induction, subjects inhaled $400 \mu \mathrm{g}$ salbutamol using a metered-dose inhaler (+spacer). Sputum was induced using an ultrasonic nebulizer (ultra-Neb 2000, Devilbiss; output set at $0.9 \mathrm{ml} / \mathrm{min}$ ). Subjects inhaled hypertonic saline $(\mathrm{NaCl} 5 \%)$ when FEV1 post salbutamol was ${ }^{3} 65 \%$ predicted and isotonic saline ( $\mathrm{NaCl} 0.9 \%$ ) when FEV1 was $<65 \%$ predicted. The aerosol was inhaled for three consecutive periods of $5 \mathrm{~min}$. FEV1 was monitored every 5 minutes for safety reasons, and when FEV1 dropped to $80 \%$ of the post-bronchodilator values, the induction procedure was stopped. The whole sputum was weighted and three volumes of PBS were added. After homogenizing by manual agitation for $30 \mathrm{sec}$ and centrifugation $(800 \mathrm{~g})$ for $10 \mathrm{~min}$ at $4^{\circ} \mathrm{C}$, the cell pellet and supernatant were separated.

Cells were treated with Sputolysinâ $0.1 \%$ (Calbiochem, Germany), washed with PBS and resuspended in $1 \mathrm{ml}$. Total cell counts, \% squamous cells and cell viability (trypan blue staining) were determined with a manual hemocytometer. Sputum cell differentials were determined by counting 500 cells non squamous cells on Cytospin samples that were stained with RAPI-DIFF II stain (Atom Scientific, Manchester, United Kingdom).

\section{Cell culture and treatments}

Primary human nasal epithelial cells (NECs) were isolated from 6 healthy volunteers and 6 patients with allergic rhinitis and asthma by gentle stroking of the inferior turbinate surface with a Rhino-Probe curette and cultured as recently described (4) in bronchial epithelial cell growth medium (Lonza). Atopy was confirmed by positive skin tests and elevated serum lgE (>100 IU/ml), and asthma was diagnosed by physicians, confirmed by positive response to bronchodilator ( $\geq 200$ cc and 12\% improvement in FEV1 and/or FVC) or a positive methacholine challenge test (PC20 $<8 \mathrm{mg} / \mathrm{ml}$ ), and had rhinitis with a sinonasal questionnaire (SNQ) score (5) of greater than 1. Healthy volunteers had no history of rhinitis or asthma, negative skin tests, negative methacholine challenge tests, and a SNQ score of less than 1. For experiments, NECs were plated on collagencoated 12-well plates at a density of $2 \times 105$ cells/well and cultured in a 1:1 mixture of bronchial epithelial cell basic medium and DMEM-H with SingleQuot supplements (Cambrex), bovine pituitary extract $(13 \mathrm{mg} / \mathrm{ml})$, bovine serum albumin $(1.5 \mu \mathrm{g} / \mathrm{ml})$, and nystatin (20 units). Following $2 \mathrm{~h}$ starvation in basal medium, NECs were treated with $50 \mathrm{ug} / \mathrm{ml}$ of HDM D. pteronyssinus; GREER, Lenoir, NC; 144.9 endotoxin units/mg protein, lot 290903) for $24 \mathrm{~h}$. Primary mouse 
tracheal epithelial (MTE) cells were isolated from wild-type (WT) C57BL/6 mice or C57BL/6 mice lacking the Inhibitor of $\mathrm{KB}$ kinase $\varepsilon$ gene (referred to herein as $/ k b k e-/-)$ and cultured as previously described $(6,7)$. After reaching confluence, MTE cells were incubated for $16 \mathrm{~h}$ in serum-free

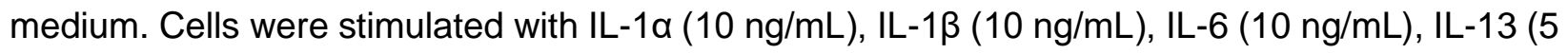
$\mathrm{ng} / \mathrm{mL}), \mathrm{IL}-33$ (5 ng/mL), TGF- $\beta 1$ ( $5 \mathrm{ng} / \mathrm{mL})$, TNF $\alpha(5 \mathrm{ng} / \mathrm{mL}), \mathrm{IL}-17$ (20 ng/mL), lipopolysaccharide (LPS, $1 \mu \mathrm{g} / \mathrm{mL})$, or HDM (10 $\mu \mathrm{g} / \mathrm{mL}$ or $50 \mu \mathrm{g} / \mathrm{mL}$ as indicated in the Figure Legend) for 2 or $24 \mathrm{hr}$.

To address the importance of glycolysis or IKKE/TBK1 in IL-1 induced pro-inflammatory responses in MTE cells, cells were pre-treated with the hexokinase inhibitor, 2- Deoxyglucose (2$\mathrm{DG}, 10 \mathrm{mM}$ ) for $1 \mathrm{~h}$ prior to exposure to IL-1 $\beta$ for $24 \mathrm{~h}$. Alternatively, cells were incubated with the lactate dehydrogenase $A$ inhibitor, oxamate $(10 \mathrm{mM})$, or the IKKE/TBK1 inhibitor, Amlexanox (10$100 \mu \mathrm{M}$, Tocris) overnight, followed by the stimulation IL-1 $\beta$ (10 $\mathrm{ng} / \mathrm{mL})$ for $24 \mathrm{~h}$. To determine whether IL-1 $\beta$-induced glycolysis or IKKع/TBK1 augmented the subsequent response to HDM, in select experiments, cells were washed post inhibitor/IL-1 $\beta$ treatment, incubated with DMEM/F12 medium for $2 \mathrm{~h}$ before stimulation with HDM for an additional 2 hours according the schematic illustrations shown in the relevant figures. To address the role of glycolysis in IL-1a-dependent proinflammatory responses, MTE cells were pre-incubated with $10 \mathrm{mM}$ 2-DG for $1 \mathrm{~h}$ or $10 \mathrm{Mm}$ oxamate for $16 \mathrm{~h}$ prior to exposure to $\mathrm{IL}-1 \mathrm{\alpha}$ for $24 \mathrm{~h}$ and subsequent assessment of proinflammatory mediators in supernatants. Note that absolute values of KC vary between studies due to freezing of some supernatants.

\section{Mouse studies}

Age-matched, 8- to 12-week-old mice were used (The Jackson Laboratory, Bar Harbor, ME) for all experiments. Wild-type (WT, C57BL6/NJ), Rag-/- (C57BL6/J), or Ikbke-/- (C57BL6/J) mice along with their strain-matched controls were sensitized (Days 1 and 8), challenged (Days 1519), and rechallenged (Days 29, 32, 36, and 39) with HDM extract containing $10 \mu \mathrm{g}$ protein (GREER, Lenoir, NC; 144.9 endotoxin units/mg protein, lot 290903) as shown in Figure 1A. Mice were euthanized and analyzed at different end points at Days 1 ( $2 \mathrm{~h}$ post HDM sensitization), 2 (24 h post HDM sensitization), 20 (24 h post the last challenge of 5 consecutive HDM challenge), 29 (2 $\mathrm{h}$ post the first HDM re-challenge), 30 (24 h post the first HDM rechallenge), and/or 40 (24 $\mathrm{h}$ post the last HDM rechallenge). The control group was subjected to saline as a vehicle control. In the Ldha siRNA knockdown studies, WT C57BL6/NJ mice were anesthetized with isoflurane and subjected to $10 \mathrm{mg} / \mathrm{kg}$ of siRNA targeting Ldha or scrambled small interfering siRNA oropharyngeally on days $26,30,33$, and 37 post-initiation of the HDM exposure regimen, and mice were harvested in day 40 (24h post the last HDM re-challenge). In the IL-1 neutralization 
experiments, WT C57BL6/J mice were challenged with HDM on days 15, 16, 17, and 18, and analyzed on day 20. Mice received $5 \mathrm{mg} / \mathrm{kg}$ of IL-1 Trap (Regeneron Pharmaceuticals, Tarrytown, NY) on Day 14 and Day 17 by i.p. injections, based upon a previous study demonstrating that this dosing regimen of IL-1 Trap attenuated cardiac remodeling after experimental acute myocardial infarction in mice (8). In select experiments, $1 \mu \mathrm{g}$ of IL-1 $\beta$ (R\&D Systems, resuspended in $0.1 \%$ BSA in PBS) was directly administered intranasally.

\section{Assessment of airway hyperresponsiveness}

Following completion of the HDM protocol, mice were anesthetized with intraperitoneal pentobarbital sodium $(90 \mathrm{mg} / \mathrm{kg})$, tracheotomized, and mechanically ventilated at 200 breaths/min. Mice were subjected to increasing doses of methacholine $(0,12.5,25,50$, and 100 $\mathrm{mg} / \mathrm{mL}$ ) administered via ultrasonic nebulization, and respiratory mechanics were assessed using a forced oscillation technique on a computer-controlled small animal ventilator (SCIREQ, QC, Canada), as previously described $(9,10)$. Parameters of Newtonian resistance (Rn), tissue resistance $(\mathrm{G})$ and elastance $(\mathrm{H})$ were calculated and quantified by averaging the three highest measurements obtained at each incremental methacholine dose for each mouse $(9,10)$.

\section{Assessment of mucus metaplasia}

Airway mucus was stained via Periodic acid Schiff (PAS) and the staining intensity was evaluated by scoring of slides by two independent blinded investigators (11). Levels of MUC5AC were evaluated in lung tissue or BAL via ELISA (My Biosource).

\section{Bronchoalveolar lavage fluid processing}

After mice were euthanized, bronchoalveolar lavage (BAL) was performed using $1 \mathrm{ml} P B S$. BAL was collected and total cell counts were determined using an Advia 120 Automated Hematology Analyzer. BAL was spun down at $1200 x g$ for 5 min. Cells were transferred to slides using a cytospin, fixed in methanol and stained using the Hema3 kit (Fisher Scientific, Kalamazoo, MI) and analyzed by counting a minimum of 300 cells per mouse, as described elsewhere (11). Supernatants were flash frozen in liquid nitrogen and stored at $-80^{\circ} \mathrm{C}$ until analysis.

\section{Enzyme-Linked Immunosorbent Assay (ELISA)}

IL-1 $\alpha$, IL-1 $\beta$, IL-6, TNFa, IL-17, GM-CSF, and CCL20 were detected by ELISA in lung homogenates (normalized for protein) or supernatants from cell culture, according to the manufacturer's instructions (R\&D Systems, Minneapolis, MN). 


\section{Quantitative Reverse Transcription-Polymerase Chain Reaction (qRT-PCR)}

RNA was extracted using miRNeasy columns (Qiagen, Valencia, CA) as directed by the manufacturer. One $\mu \mathrm{g}$ of RNA was reverse transcribed to cDNA for gene analysis using SYBR Green (Bio-Rad; Hercules, CA, USA) to assess expression of Mct4, monocarboxylate transporter 4; Pfkl, phosphofructokinase, live type; Hk2, Hexokinase 2; Glut1, glucose transporter 1; Glut3, glucose transporter 3; Pkm2, pyruvate kinase isoenzyme type M2; Ldha, lactate dehydrogenase A; Pgm1, phosphoglucomutase 1; Pdk1, pyruvate dehydrogenase kinase 1; Tpi1, triosephosphate isomerase 1; Eno1, enolase 1; Pgk1, phosphoglycerate kinase 1; Gpi, glucose6-phosphate isomerase; Pfkfb3, 6-Phosphofructo- 2-Kinase/Fructose-2,6-Biphosphatase 3; Mct1, monocarboxylate transporter 1; Glut2, glucose transporter 2; and Ikbke, inhibitory kappa B kinase $\varepsilon$. Expression values were normalized to the house keeping gene cyclophilin. Detailed primer sequences are provided in the online supplement, Table E3.

\section{Bioenergetics}

The extracellular acidification rate (ECAR) was measured using the Seahorse Extracellular Flux (XF24) Analyzer (Agilent Technologies). MTE cells were seeded onto 24-well seahorse plate at a density of 50,000 cells per well and cultured with or without $10 \mathrm{ng} / \mathrm{mL}$ IL-1 $\beta$ for $24 \mathrm{~h}$. Cells were then washed 3 times with Seahorse stress test glycolysis assay media (DMEM without glucose, L-glutamine, phenol red, sodium pyruvate, and sodium bicarbonate [Sigma-Aldrich] supplemented with $1.85 \mathrm{~g} / \mathrm{l}$ sodium chloride, $2 \mathrm{mM}$ Lglutamine, and $3 \mathrm{mg} / \mathrm{l}$ phenol red [GlycoStress Assay], $\mathrm{pH}$ 7.35). The plate was incubated in a $37^{\circ} \mathrm{C}$ non- $\mathrm{CO} 2$ incubator for $1 \mathrm{~h}$. The plate was then transferred to the Seahorse XF24 Analyzer for analysis and subjected to ECAR measurements followed by successive treatments with glucose $(10 \mathrm{mM})$, oligomycin $(0.25 \mu \mathrm{M})$, and 2-deoxyglucose (100 mM).

\section{Glucose measurements}

Glucose consumption and uptake in MTE cells were measured $24 \mathrm{~h}$ post stimulation with IL-1 $\beta$, by measuring glucose concentration in the cell culture supernatants (Eton Bioscience) and cellular incorporation of fluorescent glucose analog [2-NBDG, 2-(N-(7-Nitrobenz-2-oxa- 1,3-diazol-4yl)Amino)-2-Deoxyglucose, Life technology] using a plate reader (Biotek, Winooski, VT).

\section{Immunohistochemistry}

Fixed sections were prepared for immunostaining by deparaffinizing with xylene and rehydrating through a series of ethanol. For antigen retrieval, slides were heated for $20 \mathrm{~min}$ in $95^{\circ} \mathrm{C}$ citrate 
buffer ( $\mathrm{pH} \mathrm{6.0),} \mathrm{then} \mathrm{rinsed} \mathrm{in} \mathrm{distilled} \mathrm{water.} \mathrm{Sections} \mathrm{were} \mathrm{then} \mathrm{blocked} \mathrm{for} 1 \mathrm{~h}$ in blocking serum as per manufacturer's instructions (Vectastain Alkaline PhosphataseUniversal, Vector). Slides were then washed in TBS with $0.1 \%$ TWEEN $-203 \times 5 \mathrm{~min}$, followed by incubation with primary antibody for lactate dehydrogenase $A$ overnight at $4^{\circ} \mathrm{C}$. Sections were washed again and incubated with a biotinylated universal secondary antibody (Vectastain Alkaline Phosphatase Universal, Vector) for $30 \mathrm{~min}$ at room temperature. Slides were washed and incubated with the Vectastain ABC-AP reagent (prepared as per manufacturer's instructions) for $30 \mathrm{~min}$ at room temperature. Sections were then incubated with Vector Red/Vector Blue Alkaline Phosphatase Substrate Kit I (Vector) for $10 \mathrm{~min}$ at room temperature, rinsed with tap water, and counterstained with Mayer's Hematoxylin.

\section{Immunofluorescence}

Following euthanization, left lobes were fixed with $4 \%$ paraformaldehyde, stored at $4^{\circ} \mathrm{C}$ overnight for fixation of the tissue, mounted in paraffin, and $5 \mu \mathrm{m}$ sections were affixed to glass microscope slides for histopathology as previously described (12). For antigen retrieval, slides were heated for $20 \mathrm{~min}$ in $95^{\circ} \mathrm{C}$ citrate buffer $(\mathrm{pH} 6.0$ ) with $0.05 \%$ TWEEN- 20 then rinsed in distilled water. Sections were then blocked for $1 \mathrm{~h}$ in $1 \%$ bovine serum albumin (BSA) in PBS, followed by incubation with primary antibody for IKBKE (Cell Signaling Technology, Danvers, MA) at 1:100, overnight at $4^{\circ} \mathrm{C}$. Slides were then washed $3 \times 5 \mathrm{~min}$ in PBS, incubated with Alexafluor 647, and counterstained with DAPI in PBS for nuclear localization. Sections were imaged using a Zeiss 510-META confocal laser scanning microscope.

\section{Lactate assay}

The concentration of lactate in the medium, BAL, and lung homogenates was assessed with a Lactate Assay Kit (Eton Bioscience) according to each manufacturer's recommendations. 


\section{SUPPLEMENTAL DATA}
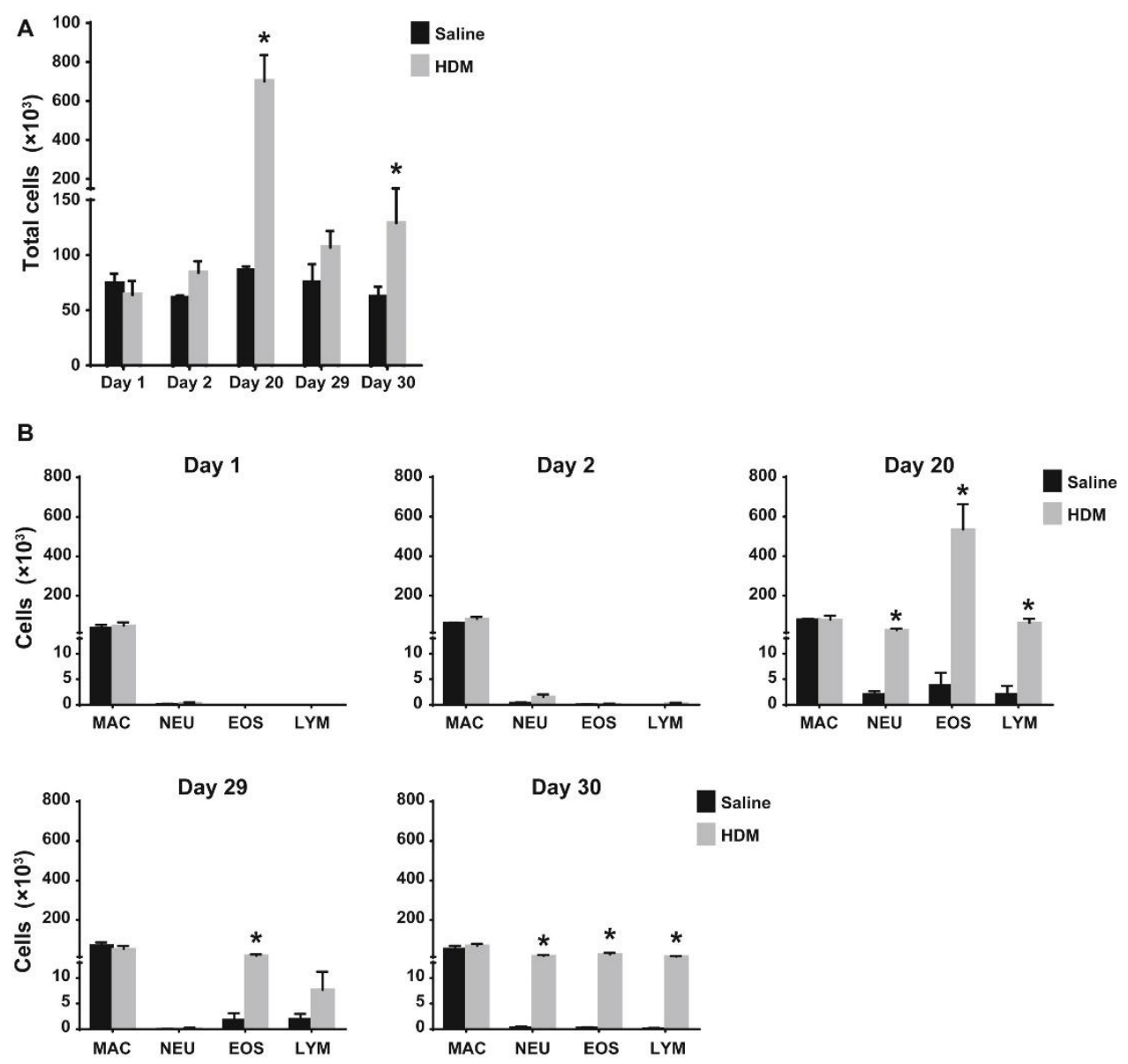

Figure E1: Airway inflammation in mice exposed to house dust mite (HDM). (A) Total and (B) differential cell counts in bronchoalveolar lavage (BAL) in response to saline or HDM, (see schematic in Figure 1A). Data are expressed as means ( \pm SEM) ( $n=5$ mice per group). * $p<$ 0.05 (Student's t test) compared with saline controls.
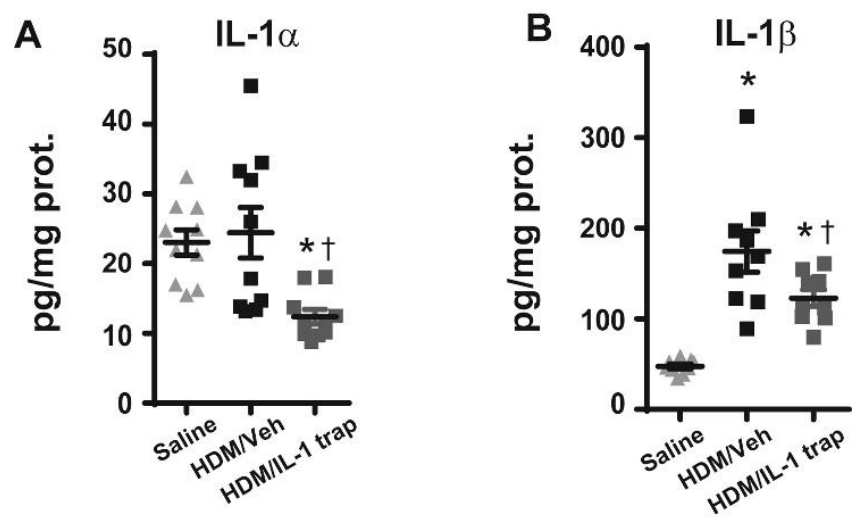
Figure E2: Assessment of levels of IL-1 $\alpha$ and IL-1 $\beta$ in lung tissues from mice subjected to vehicle control or IL1 Trap. Mice were challenged with HDM on days 15, 16, 17, and 18, and received 5 $\mathrm{mg} / \mathrm{kg}$ of IL-1 Trap or vehicle on days 14 and 17 intraperitoneally. Levels of IL-1 $\alpha$ and IL-1 $\beta$ in lung tissues homogenates were evaluated on day 20 via ELISA. ${ }^{*} P<0.05$ compared to saline control, $\uparrow p<0.05$ compared to HDM/Veh group (ANOVA).

A
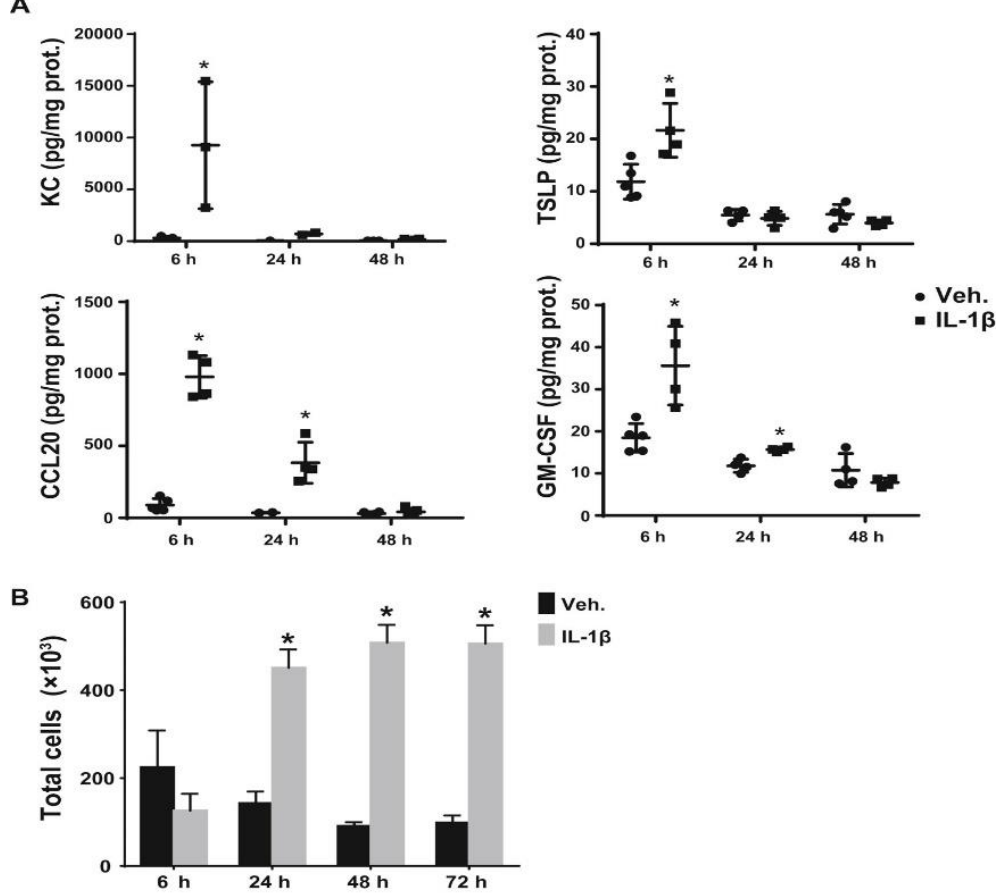

C
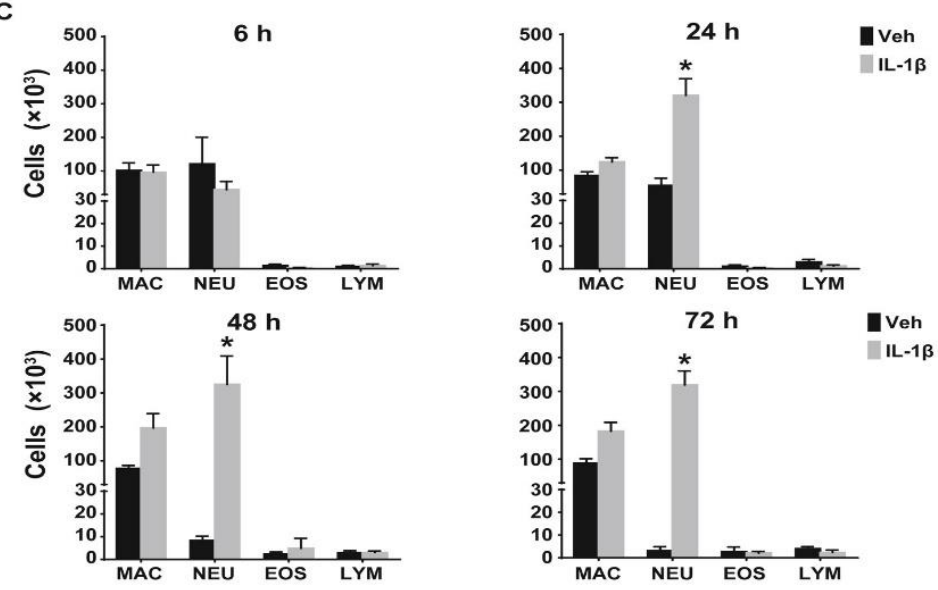

Figure E3: Evaluation of pro-inflammatory mediators and airway inflammation in mice exposed to interleukin (IL)-1 $\beta$. A: Lung tissue levels of KC, TSLP, CCL20 and GM-CSF following intranasal administration of IL-1 $\beta$ or vehicle. Total $(\mathbf{B})$ and differential cell counts $(\mathbf{C})$ in BAL from the mice at multiple time points post intranasal administration of vehicle or IL-1 1 . Data are expressed as means $\left( \pm\right.$ SEM) $\left(n=5\right.$ mice per group). ${ }^{*} p<0.05$ (ANOVA) compared with Vehicle controls. 


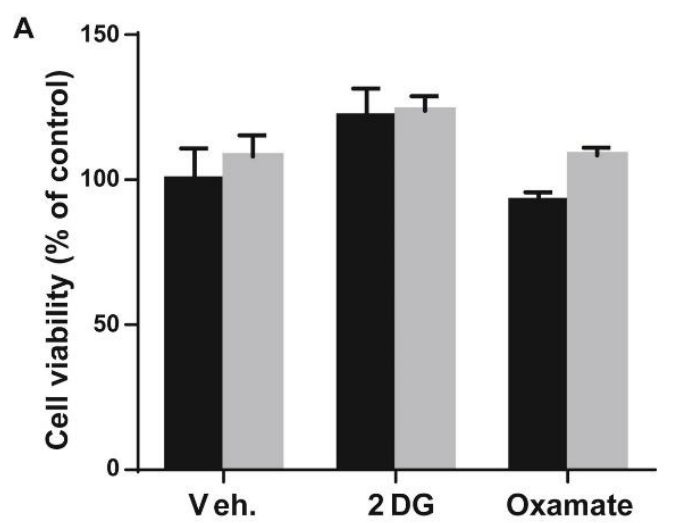

B
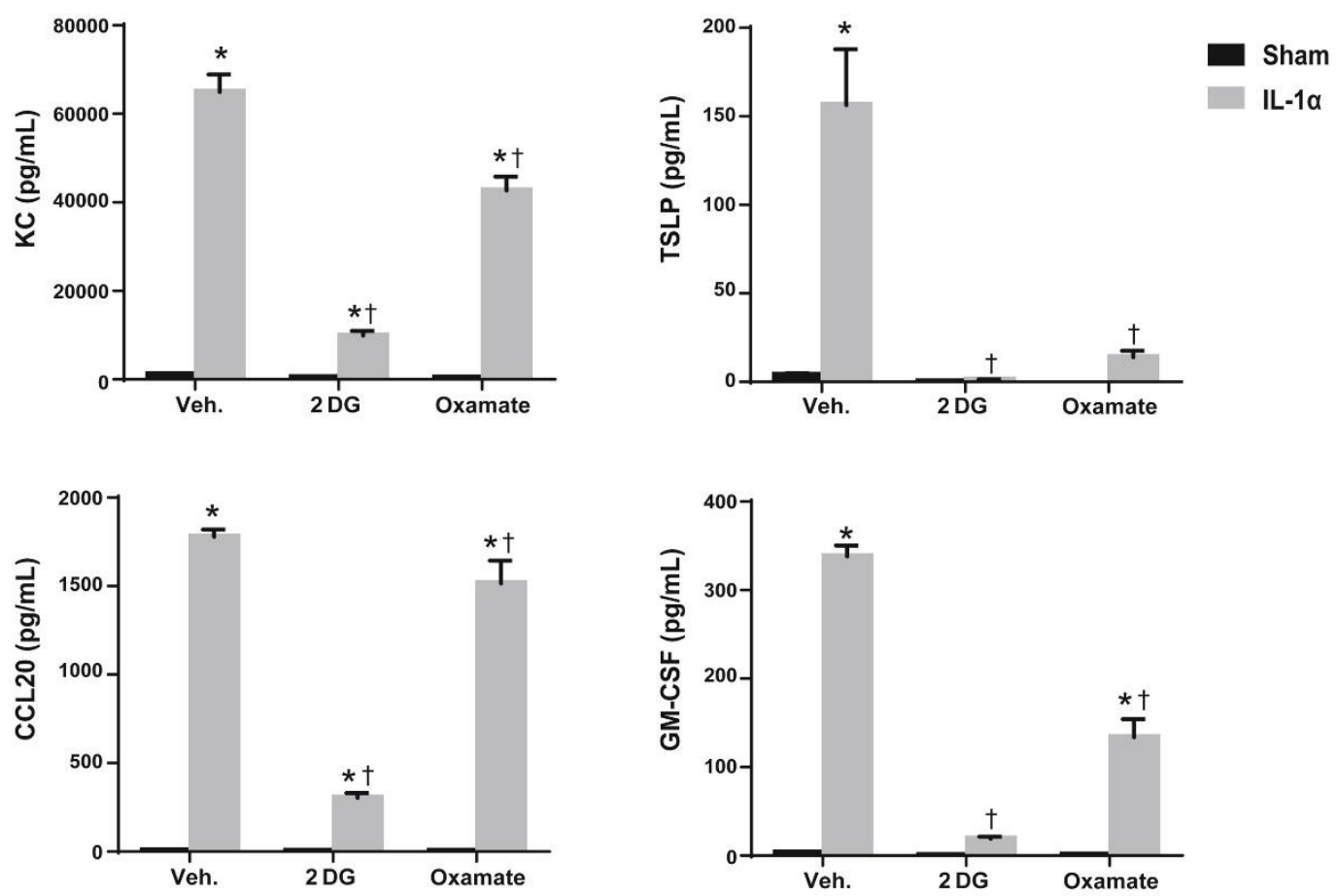

Figure E4: A: Assessment of viability following exposure to MTE cells to oxamate or 2deoxyglucose. Epithelial cells were exposed to IL-1 $\beta$ in the presence or absence of inhibitors. Cell survival was evaluated via crystal violet staining of cells. Results were expressed as \% survival compared to untreated control cultures. B: Impact of 2-DG or oxamate on IL1a-mediated increases in lactate and the indicated pro-inflammatory mediators measured $24 \mathrm{hr}$ post exposure to IL-1 $\alpha .{ }^{*} \mathrm{P}<0.05$ (ANOVA) compared to the sham group. $\dagger p<0.05$ compared to the IL-1 $\beta$ treated Vehicle group (ANOVA). 

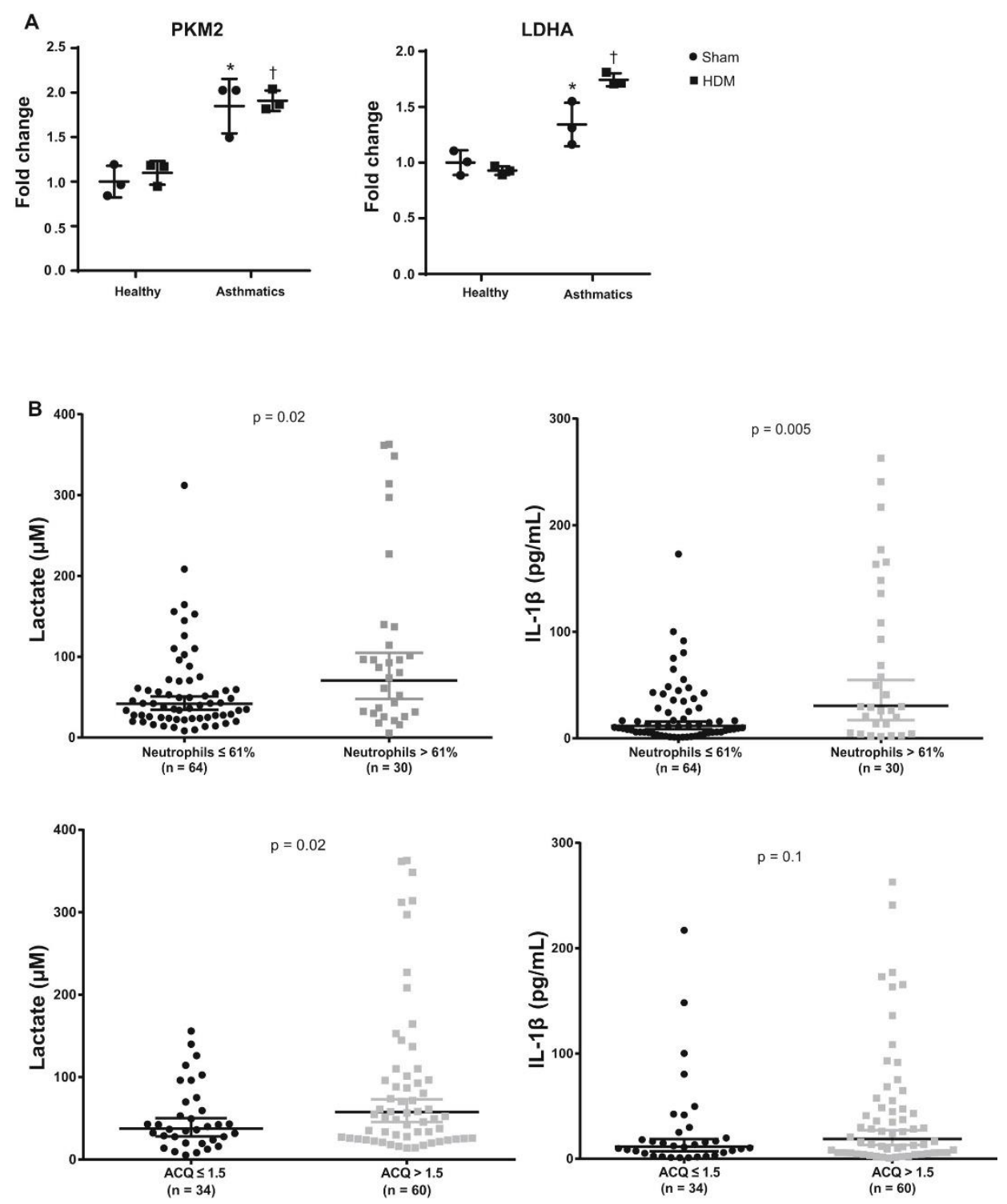

Figure E5: A: Quantification of Western blots shown in Figure 8A. Data reflects $n=3$ healthy subjects and $n=3$ asthmatics. Data were normalized to $\beta$-actin and are expressed as fold change from healthy sham controls ${ }^{*} P<0.05$ compared to the sham healthy group. $\uparrow P<0.05$ compared to HDM healthy group (ANOVA). B: Sub-analysis of sputum lactate and IL-1 $\beta$ in asthmatics with normal neutrophils (cut off $\leq 61 \%$ ) or high neutrophils (cut off $>61 \%$ ), controlled $(A C Q \leq 1.5$ ) or uncontrolled asthma (ACQ > 1.5). p-values (ANOVA or Wilcoxon rank sum test) are provided in each of the figures. 
Supplementary table I. Demographic, functional, and inflammatory characteristics of the study cohort

\begin{tabular}{llll}
\hline & Healthy subjects & Asthmatic patients & $P$ value \\
\hline $\mathrm{n}$ & 20 & 94 & \\
Age $(\mathrm{yr})$ & $53 \pm 3$ & $52 \pm 2$ & 0.86 \\
$\mathrm{BMI}(\mathrm{kg} / \mathrm{m} 2)$ & $24.30 \pm 1.05$ & $27.28 \pm 0.48$ & 0.01 \\
Age asthma onset (range) & - & $41(12-54)$ & - \\
Gender (M/F) & $10 / 10$ & $43 / 51$ & 0.3 \\
Atopy $^{*}$, no. (\%) & $4(20)$ & $42(45)$ & 0.03 \\
Positive Dpt*, no. (\% atopy) & $3(75)$ & $25(60)$ & \\
FEV1 \% predicted & $101 \pm 4.62$ & $81.56 \pm 2.07$ & 0.0001 \\
FEV1/FVC ratio & $77.68 \pm 2.51$ & $72.41 \pm 1.13$ & 0.0291 \\
FENO (ppb), median (IQR) & $20(17.3-26.8)$ & $17.5(10-36.25)$ & 0.13 \\
Eosinophils (\%), median (IQR) & $0.2(0-0.9)$ & $0.9(0.2-5.45)$ & 0.03 \\
& & & \\
Medication use & - & $5(5)$ & - \\
Not treated & - & $58(62)$ & - \\
ICS/LABA, no. (\%) & - & $1000(400-2000)$ & \\
eq Beclomethasone $\mu$ gg/ml & - & $2(2)$ & - \\
ICS alone, no. (\%) & - & $3(3)$ & - \\
OCS, no. (\%) & - & $64(68)$ & - \\
SABA, no. (\%) & - & $19(20)$ & - \\
SABA only, no. (\%) & - & $24(26)$ & - \\
LTRA, no. (\%) & & & \\
\hline
\end{tabular}

Data are expressed as means \pm SDs, or medians with interquartile range (IQR). $P$ values are based on the student's $t$ test (mean $\pm S D$ ), the $x 2$ test for proportions (sex), the Wilcoxon rank sum test (median [range]), or Poisson regression (atopy). Atopy is defined as positive test results for at least 1 specific IgE to common aeroallergens. FENO: Fraction of exhaled nitric oxide. * Dpt.: Dermatophagoid pteronyssinus. ICS: inhaled corticosteroid. LABA: long acting beta agonist. OCS: oral corticosteroids. SABA: Short acting beta agonist. LTRA: leukotriene receptor agonists. Ethnicity: All subjects are Caucasian except for 2 African subjects. 
Supplementary table II. Demographic, functional, and inflammatory characteristics of the study cohort enrolled at the University of Vermont Medical Center

\begin{tabular}{lll}
\hline & Healthy subjects & Asthmatic patients \\
\hline $\mathrm{n}$ & 7 & 6 \\
Age (range) & $23(19-27)$ & $23(19-45)$ \\
Gender (M/F) & $0 / 6$ & $3 / 4$ \\
Age asthma onset (range) & - & $4(1-18)$ \\
BMI & $23.2(19.8-26.3)$ & $28.6(22.0-35.0)$ \\
Atopy*, no. (\%) & - & $7(100)$ \\
Medication use & & \\
ICS_LABA & - & 3 \\
ICS & - & 3 \\
SABA only & - & 1 \\
SNQ & $0.4(0-0.8)$ & $1.4(1.2-1.6)$ \\
\hline
\end{tabular}

Data are expressed as median (range). ICS_LABA: inhaled corticosteroid_long acting beta agonist. ICS: inhaled corticosteroid. SABA: short acting beta agonist. SNQ: sinonasal questionnaire. *All participants were allergic to house dust mite as determined either by positive skin prick test to $D$. pteronyssinus or positive serum IgE to $D$. pteronyssinus. All subject are Caucasian.

\section{Supplementary table III. The primers used in this study}

\begin{tabular}{|c|c|c|}
\hline Genes & Forward & Reverse \\
\hline Mct4 & 5'-ATCGTGGGCACTCAGAAGTT-3' & 5'-CGCCAGGATGAACACATACTT-3' \\
\hline$P f k l$ & 5'-CATATATGTGGGGGCCAAAG-3' & 5'-GACACACAGGTTGGTGATGC-3' \\
\hline$H k 2$ & 5'-GGGACGACGGTACACTCAAT-3' & 5'-GCCAGTGGTAAGGAGCTCTG-3' \\
\hline Glut1 & 5'-TCTCTGTCGGCCTCTTTGTT-3' & 5'-CCAGTTTGGAGAAGCCCATA-3' \\
\hline Glut2 & 5'-GCCTGTGTATGCAACCATTG-3' & 5'-GAAGATGGCAGTCATGCTCA-3' \\
\hline Glut3 & 5'-TGTCACAGGAGAAGCAGGTG-3' & 5'-GCTCCAATCGTGGCATAGAT-3' \\
\hline Pkm2 & 5'-CTGCAGGTGAAGGAGAAAGG-3' & AGATGCAAACACCATGTCCA-3' \\
\hline Ldha & 5'-GGAAGGAGGTTCACAAGCAG-3' & 5'-ACCCGCCTAAGGTTCTTCAT-3' \\
\hline Pgm1 & 5'-TCAGGCCATTGAGGAAAATC-3' & 5'-CGAACTTCACCTTGCTCTCC-3' \\
\hline Pdk1 & 5'-GGCGGCTTTGTGATTTGTAT-3' & 5'-ACCTGAATCGGGGGATAAAC-3' \\
\hline Tpi1 & 5'-CCTGGCCTATGAACCTGTGT-3' & 5'-CAGGTTGCTCCAGTCACAGA-3' \\
\hline Eno1 & 5'-CTGCCTCCGAGTTCTACAGG-3' & 5'-CGCTTAGGGTTGGTCACTGT-3' \\
\hline Pgk1 & 5'-CAAGGCTITGGAGAGTCCAG-3' & 5'-TGTGCCAATCTCCATGTTGT-3' \\
\hline Gpi & 5'-GTGGTCAGCCATTGGACTTT-3' & 5'-CTGGAAATAGGCAGCAAAGC-3' \\
\hline Pfkfb3 & 5'-CAGCTACCAGCCTCTTGACC-3' & 5'-AACTTCTTGCCTCTGCTGGA-3' \\
\hline Mct1 & 5'-TCCAGTAATGATCGCTGGTG-3' & 5'-AGTTGAAAGCAAGCCCAAGA-3' \\
\hline Ikbke & 5'-CTGGATGTCCCAAAGTTCGT-3' & 5'-AGGCTGCTGCTGAGGTAGAG-3' \\
\hline
\end{tabular}




\section{REFERENCES}

1. Manise $M$, Holtappels $G$, Van Crombruggen $K$, Schleich F, Bachert $C$, Louis $R$. Sputum IgE and cytokines in asthma: relationship with sputum cellular profile. PloS one. 2013;8(3):e58388.

2. Delvaux $M$, Henket $M$, Lau L, Kange P, Bartsch P, Djukanovic $R$, et al. Nebulised salbutamol administered during sputum induction improves bronchoprotection in patients with asthma. Thorax. 2004;59(2):111-5.

3. Maes T, Cobos FA, Schleich F, Sorbello V, Henket M, De Preter $K$, et al. Asthma inflammatory phenotypes show differential microRNA expression in sputum. Journal of Allergy and Clinical Immunology. 2016;137(5):1433-46.

4. Muller L, Brighton LE, Carson JL, Fischer WA. Culturing of human nasal epithelial cells at the air liquid interface. Journal of visualized experiments: JoVE. 2013(80).

5. Dixon AE, Sugar EA, Zinreich SJ, Slavin RG, Corren J, Naclerio RM, et al. Criteria to screen for chronic sinonasal disease. CHEST Journal. 2009;136(5):1324-32.

6. Wu R, Smith D. Continuous multiplication of rabbit tracheal epithelial cells in a defined, hormonesupplemented medium. In vitro. 1982;18(9):800-12.

7. Alcorn JF, Guala AS, van der Velden J, McElhinney B, Irvin CG, Davis RJ, et al. Jun N-terminal kinase 1 regulates epithelial-to-mesenchymal transition induced by TGF-B1. Journal of cell science. 2008;121(7):1036-45.

8. Van Tassell BW, Varma A, Salloum FN, Das A, Seropian IM, Toldo S, et al. Interleukin-1 trap attenuates cardiac remodeling after experimental acute myocardial infarction in mice. Journal of cardiovascular pharmacology. 2010;55(2):117-22.

9. Tomioka S, Bates JH, Irvin CG. Airway and tissue mechanics in a murine model of asthma: alveolar capsule vs. forced oscillations. Journal of Applied Physiology. 2002;93(1):263-70.

10. Riesenfeld E, Allen GB, Bates JH, Poynter ME, Wu M, Aimiand S, et al. The temporal evolution of airways hyperresponsiveness and inflammation. Journal of allergy \& therapy. 2012;1(5):1.

11. Tully JE, Hoffman SM, Lahue KG, Nolin JD, Anathy V, Lundblad LK, et al. Epithelial NF-KB orchestrates house dust mite-induced airway inflammation, hyperresponsiveness, and fibrotic remodeling. The Journal of Immunology. 2013;191(12):5811-21.

12. Anathy V, Aesif SW, Hoffman SM, Bement JL, Guala AS, Lahue KG, et al. Glutaredoxin-1 attenuates Sglutathionylation of the death receptor fas and decreases resolution of Pseudomonas aeruginosa pneumonia. American journal of respiratory and critical care medicine. 2014;189(4):463- 74. 


\section{CHAPTER 4:}

Activation of Pyruvate Kinase M2 attenuates expression of pro-inflammatory mediators in house dust mite-induced allergic airways disease

van de Wetering $C^{*}$, Aboushousha $\mathrm{R}^{\star}$, Manuel AM, Chia SB, Erickson C, MacPherson

MB, van der Velden JL, Anathy V, Dixon AE, Irvin CG, Poynter ME, van der Vliet A, Wouters EFM, Reynaert NL, Janssen-Heininger YMW. * Equal contribution

J Immunol. 2020 Feb 15;204(4):763-774. 


\section{ABSTRACT}

Asthma is a chronic disorder characterized by inflammation, mucus metaplasia, airway remodeling and hyperresponsiveness. We recently showed that interleukin-1 (IL-1)-induced glycolytic reprogramming contributes to allergic airway disease using a murine house dust mite (HDM) model. Moreover, levels of pyruvate kinase M2 (PKM2) were increased in this model as well as in nasal epithelial cells from asthmatics as compared to healthy controls. While the tetramer form of PKM2 converts phosphoenolpyruvate to pyruvate, the dimeric form of PKM2 has alternative, non-glycolysis functions as a transcriptional co-activator to enhance the transcription of several pro-inflammatory cytokines. In the present study, we examined the impact of PKM2 on the pathogenesis of HDM-induced allergic airways disease in C57BI/6NJ mice. We report here that activation of PKM2, using the small molecule activator, TEPP46, augmented PKM activity in lung tissues and attenuated airway eosinophils, mucus metaplasia, and subepithelial collagen. TEPP46 attenuated IL-1b-mediated airway inflammation and expression of pro-inflammatory mediators. Exposure to TEPP46 strongly decreased the IL-1b-mediated increases in thymic stromal lymphopoietin (TSLP) and granulocyte macrophage colony stimulating factor (GMCSF), in primary tracheal epithelial cells isolated from C57BI/6NJ mice. We also demonstrate that IL-1bmediated increases in nuclear phospho-STAT3 were decreased by TEPP46. Lastly, STAT3 inhibition attenuated the IL-1b-induced release of TSLP and GM-CSF, suggesting that the ability of PKM2 to phosphorylate STAT3 contributes to its pro-inflammatory function. Collectively, these results demonstrate that the glycolysis-inactive form of PKM2 plays a crucial role in the pathogenesis of allergic airways disease by increasing IL-1b-induced proinflammatory signaling, in part through phosphorylation of STAT3.

\section{Key Points:}

- A small molecular activator of PKM2 attenuates allergic airways disease in mice.

- Activation of PKM2 decreases IL-1b-induced airway inflammation.

- PKM2 activation decreases IL-1b-induced nuclear phosphorylation of STAT3.

\section{Keywords:}

Asthma, Pyruvate Kinase M2, Interleukin-1ß, TEPP46, Thymic stromal lymphopoietin, Granulocyte macrophage colony stimulating factor

\section{Abbreviations}

BAL: bronchoalveolar lavage; EGF: epidermal growth factor; EGFR: EGF receptor; GLUT-1:

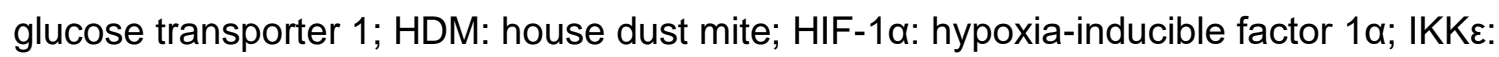
inhibitory k B kinase $\varepsilon$; MTE: mouse tracheal epithelial; PEP: phosphoenolpyruvate; PK: 
pyruvate kinase; PKM1: PK muscle isozyme M1; PKM2: PK muscle isozyme M2; a-SMA: asmooth muscle actin; TEPP46: 6-[(3-Aminophenyl)methyl]-4,6-dihydro-4-methyl2(methylsulfinyl)-5H-Thieno[29,39:4,5]pyrrolo[2,3-d]pyridazin-5-one; TSLP: thymic stromal lymphopoietin; WT: wild-type.

\section{INTRODUCTION}

Asthma is a complex pulmonary disorder that is characterized by mucus metaplasia, airways hyperresponsiveness (AHR) and remodeling, and is accompanied by a chronic inflammatory process controlled by cells of the innate and adaptive immune system (1). The precise metabolic alterations that are induced in structural or immune cells which promote the disease processes remain incompletely understood. However, glycolytic reprogramming has been shown to be important in the regulation of immune cell activation and differentiation $(1,2)$. Our laboratory recently described that interleukin-1 (IL-1)-induced glycolytic reprogramming contributes to allergic inflammation, airway remodeling and AHR in a mouse model of house dust mite (HDM)induced allergic airway disease (3). Moreover, enhanced glycolysis was shown to be required for the IL-1 $\beta$-mediated release of the pleiotropic cytokines thymic stromal lymphopoietin (TSLP) and granulocyte macrophage colony stimulating factor (GM-CSF), two major epithelium-derived inflammatory mediators implicated in the pathogenesis of asthma. Levels of lactate were also increased in sputum of asthmatics, and significant correlations were observed between lactate and IL-1 $\beta$. Moreover, lactate levels were elevated in subjects with neutrophilic asthma who had poor disease control (3), suggesting that increased glycolysis may be feature of severe asthma.

During glycolysis, glucose is converted into pyruvate which can be further metabolized in the mitochondria to produce adenosine triphosphate (ATP) via oxidative phosphorylation. Pyruvate kinase $(P K)$ catalyzes the final, rate-limiting step in glycolysis, the formation of pyruvate from phosphoenolpyruvate (PEP) while generating two molecules of ATP per glucose molecule. Pyruvate can also be converted into lactate under hypoxic conditions (anaerobic glycolysis), or in the presence of oxygen (aerobic glycolysis) in metabolically active cells such as cancer cells (4, 5). The PK family consists of four isoforms, which are encoded by two distinct genes. The Pkrl gene encodes the isoforms PKL and PKR, which are expressed in the liver and red blood cells respectively, and the PK muscle isozymes M1 and M2 (PKM1 and PKM2) which are derived from alternative splicing of the PKM gene $(6,7)$. PKM1 naturally occurs in a highly active tetrameric form, and is expressed in many differentiated tissues such as the muscle and the brain (8), whereas PKM2 can adopt monomer, dimer or tetramer structural forms that dictate its intracellular function $(9,10)$. PKM2 is highly expressed during embryonic development as well as in 
proliferating cells (9). Tetrameric PKM2 has a high binding affinity to its substrate, PEP, prompting PKM2 glycolytic activity (11). In contrast, PKM2 in its dimer form has a low binding affinity to PEP, and can translocate into the nucleus where it acts as a transcriptional co-activator to enhance transcription of multiple pro-inflammatory cytokines (12). PKM2 has been shown to phosphorylate signal transducer and activator of transcription 3 (STAT3), which in turn augments its transcriptional activity (13). PKM2-linked STAT3 activation was recently shown to contribute to LPS-induced lung injury (14).

We previously showed that in mice with HDM-induced airway disease, levels of pyruvate kinase M2 were increased, compared to controls. Similarly, primary nasal epithelial cells derived from asthmatics also displayed increased PKM2 protein levels, compared to cells from healthy controls. These observations of increases in PKM2 in settings of allergic airway disease along with its dichotomous role as a glycolysis enzyme (glycolytic kinase) or proinflammatory mediator, led us to investigate whether a small molecule activator of PKM2, which stabilizes tetrameric PKM2 to promote conversion of PEP to pyruvate, affects HDM-induced allergic airways disease and IL-1 $\beta$-induced inflammation.

Here we show that activation of the glycolysis function of PKM2 with the small molecule activator, TEPP46, exerts an anti-inflammatory effect in models of HDM- or IL-1 $\beta$-induced lung inflammation in association with diminished activation of STAT3. 


\section{MATERIALS AND METHODS}

\section{Reagents and antibodies}

All reagents were from Sigma-Aldrich unless otherwise noted.

\section{Mouse studies}

Age-matched 8-10 weeks old male and female wild-type C57BI/6NJ mice (WT mice) were bred at the University of Vermont. All animal experiments were approved by the Institutional Animal Care and Use Committee. To induce allergic airways disease, mice were sensitized intranasally with $10 \mu \mathrm{g}$ of HDM (GREER, Lenoir, NC, XPB70D3A2.5, lot 348718; volume: $2.5 \mathrm{~mL} /$ vial; endotoxin: 1140 EU/vial; Der p 1 levels: 144.9 mcg/vial; dry weight: 17 mg/vial; protein 2.92 $\mathrm{mg} / \mathrm{vial}$ ) in week 1 (day 1), re-sensitized in week 2 (day 8) followed by 5 consecutive challenges in week 3 (day 15-19). Moreover, mice were intraperitoneally (i.p.) injected once per day with 25 $\mathrm{mg} / \mathrm{kg}$ or $50 \mathrm{mg} / \mathrm{kg}$ TEPP46 (6-[(3-Aminophenyl)methyl]-4,6-dihydro-4-methyl-2-(methylsulfinyl)5H-Thieno[2',3':4,5]pyrrolo[2,3-d]pyridazin-5-one, Cayman Chemicals) on days 14-19. HDM was dissolved in saline, whereas TEPP46 was dissolved in 100\% DMSO and further diluted 1:1 in a $0.5 \%$ carboxy methyl cellulose solution in water. Therefore, the vehicle control groups were exposed to saline and received DMSO (50\%) in carboxy methyl cellulose solution (50\%). Mice were harvested on day 20, 24 hours after the last HDM installation. In separate experiments, WT mice were intraperitoneal injected with $50 \mathrm{mg} / \mathrm{kg}$ TEPP46 at day 1, followed by a second injection after 24 hours of $50 \mathrm{mg} / \mathrm{kg}$ TEPP46 at day 2, together with intranasal administration of $1 \mu \mathrm{g}$ of IL$1 \beta$ (R\&D Systems). IL-1 $\beta$ was dissolved in $0.1 \%$ Bovine Serum Albumin (BSA) in Phosphate Buffered Saline (PBS). Mice were harvested 6 or 24 hours after the IL-1 $\beta$ administration.

\section{Bronchoalveolar lavage (BAL) fluid processing}

Mice were euthanized, and BAL was performed using $1 \mathrm{~mL}$ PBS. BAL was collected and total cells were counted manually using a hemocytometer. BAL was centrifuged at 500xg for 10 minutes at $4^{\circ} \mathrm{C}$. Supernatant was stored at $-80^{\circ} \mathrm{C}$ for further analysis. The cells were resuspended in 5\% BSA in PBS and subsequently transferred to slides using a cytospin, fixed in $100 \%$ methanol for 5 minutes and stained with the Hema3 kit (Fisher Scientific, Kalamazoo, MI). Total macrophages, neutrophils, eosinophils, and lymphocytes were analyzed by counting a total of 300 cells per slide by two independent investigators blinded to the identity of the samples.

\section{Cell culture}


Primary mouse tracheal epithelial cells (MTE) were isolated from WT C57BL/6NJ mice (purchased from The Jackson Laboratory, Bar Harbor, ME) and cultured as previously described $(15,16)$. WT MTE cells were grown on 12-well transwell inserts to confluency, followed by overnight starvation, and pre-treated with $100 \mu \mathrm{M}$ TEPP46 (Cayman Chemicals) for 1 hour prior to stimulation with $10 \mathrm{ng} / \mathrm{mL} \mathrm{IL}-1 \beta$ (R\&D Systems) for $24 \mathrm{hrs}$. In all cell experiments, MTE cells were treated at the apical and basolateral side. In HDM-treated MTE cell experiments, cells were washed 24 hours after IL-1 $\beta$ treatment, incubated with DMEM/F12 medium for 2 hours before stimulation with $50 \mu \mathrm{M}$ HDM (GREER, Lenoir, NC, XPB70D3A2.5, lot 348718; volume: 2.5 $\mathrm{mL} / \mathrm{vial}$; endotoxin: $1140 \mathrm{EU} / \mathrm{vial}$, Der p 1 levels: $144.9 \mathrm{mcg} / \mathrm{vial}$; dry weight: $17 \mathrm{mg} / \mathrm{vial}$; protein $2.92 \mathrm{mg} / \mathrm{vial}$ ) for an additional 2 hours. Cells were harvested for protein or mRNA, and medium was collected for analysis of lactate and cytokine levels. To examine the contribution of phosphorylation of STAT3, MTE cells were treated with Stattic (17) (Abcam, Cambridge, UK) at the indicated concentrations for 1 hour prior to IL-1 $\beta$ treatment for 24 hrs.

\section{Assessment of mucus metaplasia and collagen deposition}

Left lung lobes were fixed in 4\% paraformaldehyde in PBS, embedded in paraffin, and sectioned. Airway mucus was analyzed via Periodic acid Schiff (PAS) stain. Collagen deposition was assessed via Masson's trichrome stain. The intensity of the staining was evaluated by scoring of slides by two independent blinded investigators.

\section{Western blotting}

Protein concentrations in cell and tissue lysates were determined by Bio-Rad DC Protein Assay Kit (Bio-Rad, Hercules, CA). Proteins were resolved using reducing sodium dodecyl sulfate (SDS)-polyacrylamide gel electrophoresis, then transferred to polyvinylidene difluoride (PVDF) membranes followed by incubation with the indicated primary antibody. PKM1 (\#7067), PKM2 (\#4053), p-STAT3 (\#8119), STAT3 (\#4904), IKKE (\#3416) and histone H3 (\#4499) antibodies were obtained from Cell Signaling Technology (Danvers, MA, USA). $\beta$-actin antibody was acquired from Sigma-Aldrich. Subsequently, membranes were incubated with peroxidaseconjugated secondary antibodies and visualized using chemiluminescence (Pierce, Rockford, IL, USA). Non-reducing gel electrophoresis assays in the presence of the disuccinimidyl suberate (DSS) crosslinker (Thermo Scientific, MA, USA) were performed to evaluate the formation of tetrameric PKM2. Densitometric analyses were performed using Image J Software. Values were normalized to corresponding $\beta$-actin bands.

\section{PKM activity assay}


PKM activity assay was performed using a pyruvate kinase activity kit according to the manufacturer's protocol (BioVision, CA, USA). Briefly, lung tissues or MTE cells were homogenized in PBS and lysates were normalized to equal protein concentrations. Equal volumes (total volume of $50 \mu \mathrm{l}$ ) of normalized lung tissue or cell lysates were used in the assay. The relative fluorescence units (RFU), which displays the rate of pyruvate yield, was normalized and expressed as RFU per minute per $\mu \mathrm{g}$ of protein.

\section{Preparation of nuclear extracts}

For fractionation, MTE cells were stimulated with $10 \mathrm{ng} / \mathrm{mL}$ IL-1 $\beta$ (R\&D Systems) for 24 hours. Fractionation of nuclear and cytosolic extracts was performed by using the Nuclear and Cytoplasmic Extraction kit (Thermo Scientific, MA, USA) according to manufacturer's protocol followed by western blotting.

\section{Lactate measurements}

Lactate levels were measured in cell culture medium with a lactate assay kit (Eton BioScience) according to manufacturer's instructions. Equal volumes of cell culture medium was used in 10 $\mathrm{kDa}$ Amicon Ultra centrifugal filters (EMD-Millipore). Samples were deproteinized by centrifugation for 1 hour at $14000 x g$ at $4^{\circ} \mathrm{C}$.

\section{Cell viability assay}

MTE cells were gently washed twice in ice cold PBS and stained with a Crystal Violet dye $(0.5 \%$ Crystal Violet solution in $20 \%$ methanol) for 20 minutes at room temperature. After incubation, the staining solution was carefully removed and the cells were washed 4 times with distilled water. Subsequently, $10 \%$ acetic acid was added to the cells for 30 seconds while shaking. Lastly, 100 $\mu \mathrm{l}$ of the acetic acid solution per well was transferred to a 96 wells plate and the optical density was measured at a wavelength of $595 \mathrm{~nm}$. In addition, a Calcein AM assay kit (Cayman Chemicals) was used according to manufacturer's instructions. Cell survival was expressed as percentage of survival compared to untreated control cultures.

\section{Real-Time quantitative PCR (Q-PCR)}

Total RNA was extracted using miRNeasy columns (Qiagen, Valencia, CA) according to the manufacturer's protocol. First-strand cDNA was synthesized from $1 \mu \mathrm{Rg}$ RNA and reverse transcribed for gene analysis using SYBR Green (Bio-rad, Hercules, CA). cDNA from the samples were amplified by real-time quantitative PCR (Q-PCR) with specific primers for Ts/p, Csf2, Cxcl1, 
Ccl20, Muc5AC, and Col1a1. The data was normalized to Ppia (also known as cyclophilin A). The primer sequences are listed in supplementary table I.

\section{Enzyme-linked immunosorbent assay (ELISA)}

CCL20, TSLP, GM-CSF, KC, IL-33, and IL-1 $\beta$ were detected by enzyme-linked immunosorbent assay (ELISA) kits (R\&D systems, Minneapolis, MN) in normalized lung tissue lysates, or supernatants from cell cultures according to the manufacturer's instructions.

\section{Statistical analysis}

Data are expressed as means \pm SEM. All cell experiments were performed at least 3 times with $\mathrm{n}=3$ per group. Significant differences between groups were determined using the GraphPad Prism software (Graphpad) by two-way ANOVA with a Tukey post hoc test for multiple comparisons. $\mathrm{P}$ values lower than 0.05 were accepted as significant. 


\section{RESULTS}

Activation of the glycolysis function of PKM2 with TEPP46 attenuates airway inflammation, mucus metaplasia, and subepithelial collagen in mice with HDM-induced allergic airways disease

To investigate the role of PKM2 in the pathogenesis of HDM-induced allergic airways disease, C57BL6/NJ mice were sensitized with HDM once in week 1, and in week 2, followed by 5 consecutive challenges in week 3 to induce allergic airways disease (Figure 1A). To promote PKM2 glycolytic activity, mice were injected intraperitoneally once per day with TEPP46 during the HDM challenges (days 14-19), starting the day prior to the HDM challenges in week 3 (Figure 1A). Administration of TEPP46 resulted in elevated activity of PKM in lung tissue from saline control animals and further increases in PKM activity were observed in lungs from mice exposed to HDM, indicating that TEPP46 augmented PKM2 activity (Figure 1B). While protein levels of PKM1 did not differ between the groups, we observed slight increases in PKM2 expression in HDM-exposed mice (Figure 1C), consistent with our previous observations, and these increases in PKM2 expression were no longer observed in mice receiving TEPP46. No statistically significant differences in overall PKM activity were observed in lungs from saline or HDM-treated mice in the absence of TEPP46 (Figure 1B, vehicle groups), despite observed increases in PKM2 expression in mice exposed to HDM (Figure 1C), suggesting that the increased expression of PKM2 does not contribute to its enhanced activity as a glycolysis enzyme, converting PEP to pyruvate. Next, we assessed the extent of inflammation by total and differential immune cell counts in the BALF. Activation of PKM2 by TEPP46 attenuated the HDM-mediated increases in total cells in the BALF, reflected by a decrease in eosinophils (Figure 1D, E), while the number of neutrophils, macrophages and lymphocytes were comparable between the HDM-treated groups. To further investigate the impact of activation of PKM2 on the extent of HDM-induced allergic airway inflammation, we evaluated protein levels of various cytokines in lung tissue homogenates. HDM-mediated increases of the cytokines CCL20 and KC, were attenuated upon PKM2 activation (Figure 1F). Small, but not statistically significant, decreases in IL-33 were observed in HDM exposed mice that received TEPP46, compared to the respective control group while IL-1 $\beta$ levels did not change (Figure 1F). Together, these results show that PKM2 activation, using TEPP46, attenuates HDM-induced inflammatory cytokines in the lung. 
A

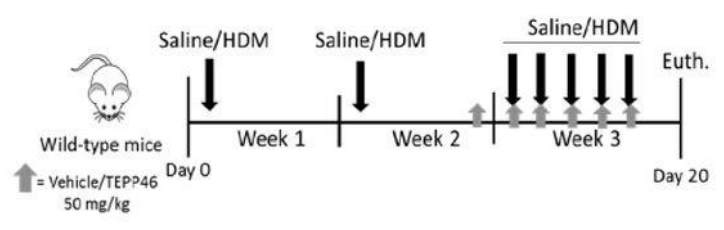

C

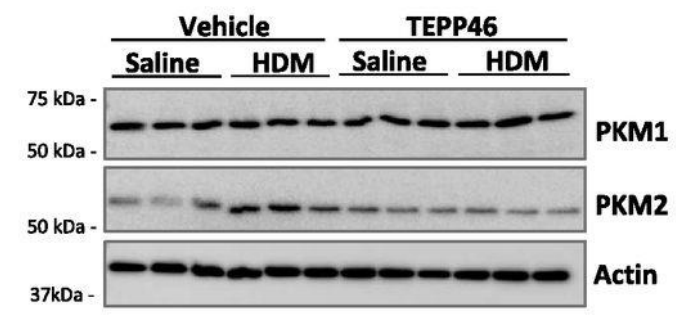

D

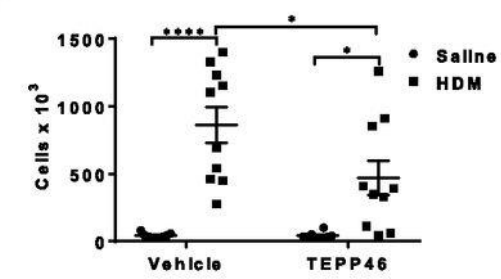

$\mathrm{F}$
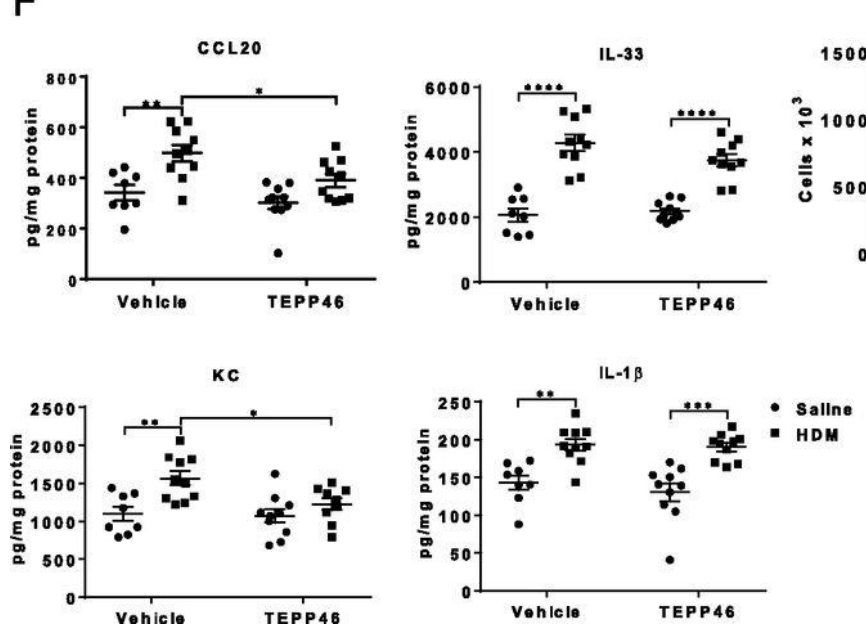
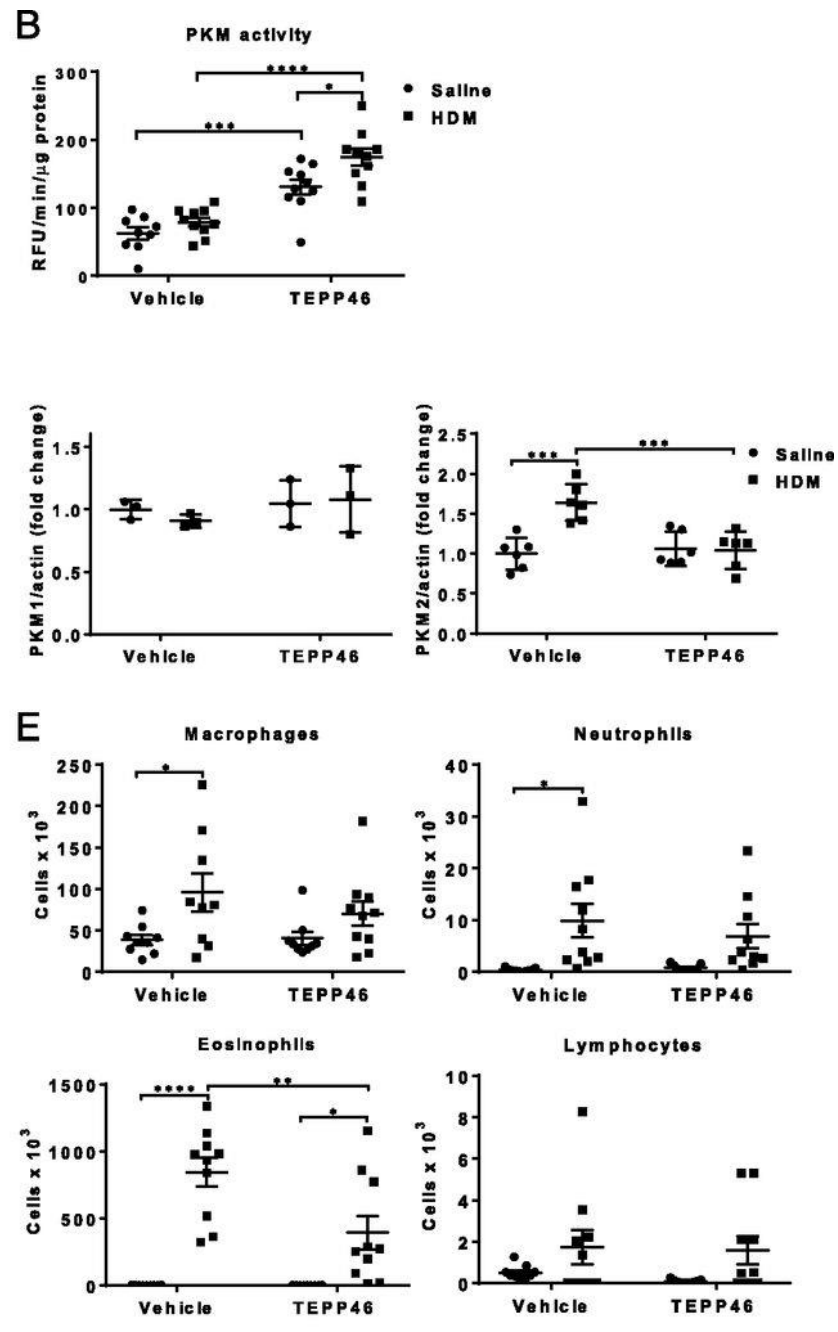

Figure 1: Activation of PKM2 by TEPP46 attenuates pro-inflammatory cytokines in mice with HDM-

induced allergic airway disease. A, Schematic depicting the exposure regimen. Mice were sensitized twice with $10 \mu \mathrm{g}$ of HDM or saline on days 1 , and 8 . Mice were treated with $50 \mathrm{mg} / \mathrm{kg}$ TEPP46 intraperitoneally daily, starting on day 14. Mice were challenged with HDM on days 15- 19 and euthanized 24 hours after the final HDM challenge. B, Assessment of PKM activity in lung tissue homogenates. C, Representative western blots and quantification for total PKM1, and PKM2 levels. b-actin; loading control. n=3-6 per group. D and E, Total and differential cell counts in bronchoalveolar lavage fluid. $F$, Measurements of CCL20, IL-33, KC, and IL-1b in lung tissue homogenates by ELISA. For A-B, D-F; $n=8$ 10 per group. ${ }^{*} \mathrm{P}<0.05 ;{ }^{* \star} \mathrm{P}<0.01 ;{ }^{* \star *} \mathrm{P}<0.001 ;{ }^{* * *} \mathrm{P}<0.0001$. 
We next evaluated the impact of PKM2 activation on airway remodeling, by assessing mucus metaplasia, subepithelial collagen and alpha smooth muscle actin ( $\alpha-S M A)$. Results in Figure 2AE demonstrate that administration of TEPP46 attenuated HDM-mediated increases in mucus metaplasia, subepithelial collagen and a-SMA content, and decreased expression of Muc5AC, and Col1a1 mRNAs. These results demonstrate that activation of PKM2 by TEPP46 attenuates airway remodeling in mice with HDM-induced allergic airways disease.
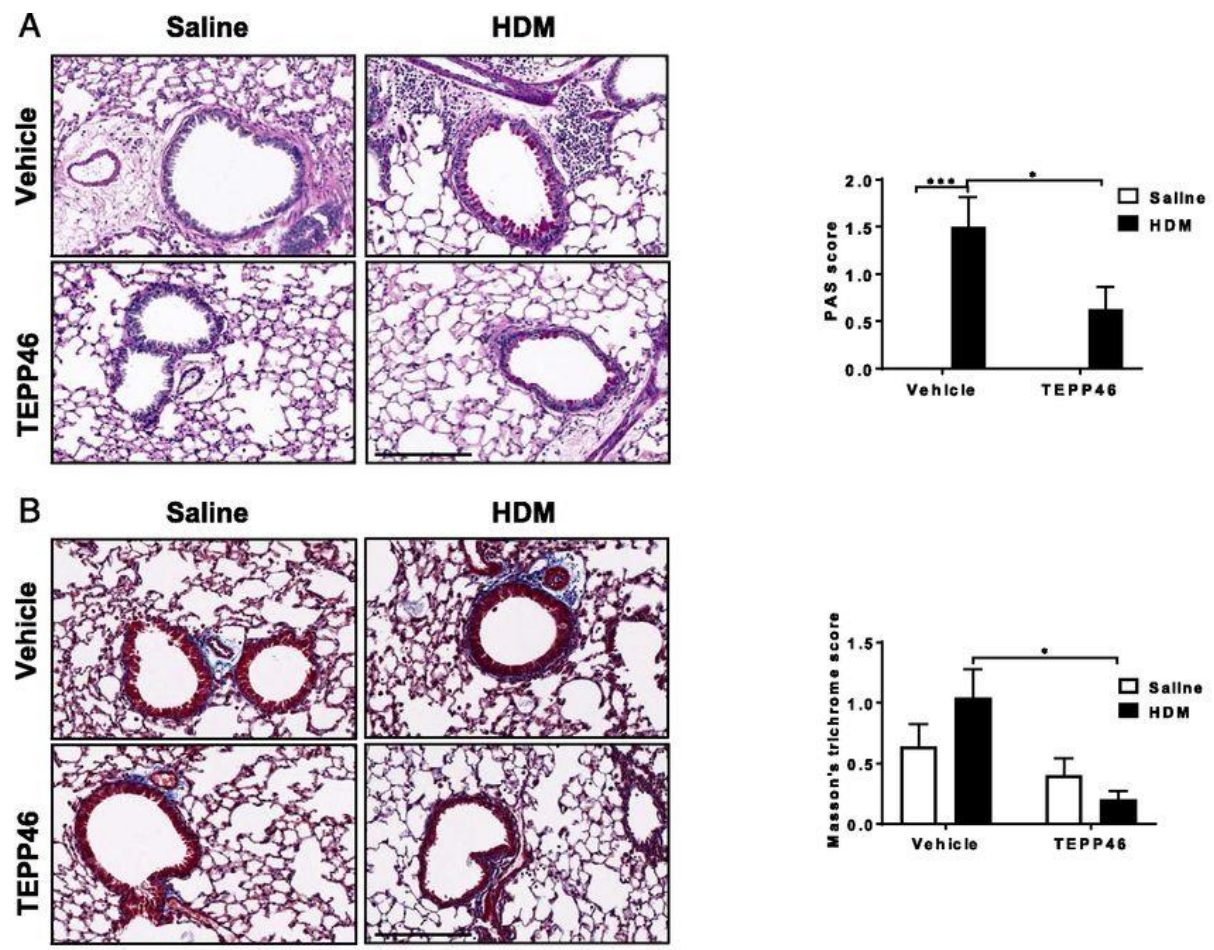

C

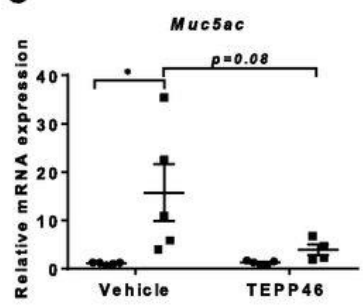

$\mathrm{E}$

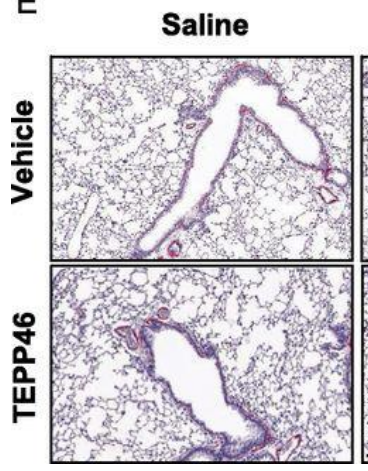

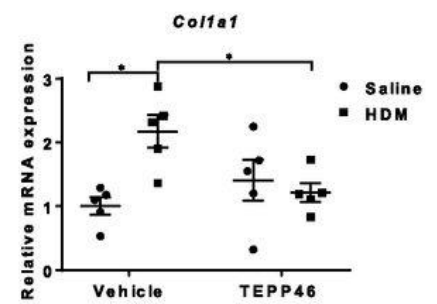

HDM

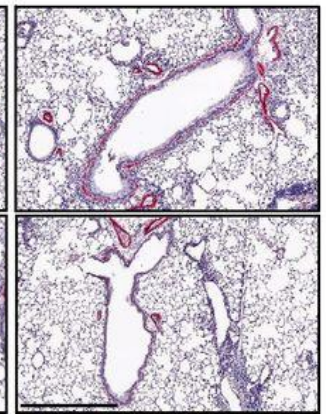

D

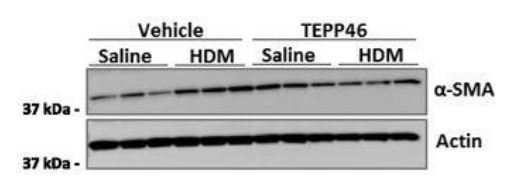


Figure 2: Activation of PKM2 by TEPP46 attenuates mucus metaplasia, subepithelial collagen, and markers of airway remodeling in mice with HDM-induced allergic airway disease. A, Assessment and quantification of mucus metaplasia by PAS staining intensity and B, Collagen deposition by Masson's trichrome staining. Scale bars: $200 \mu \mathrm{m}$. C, mRNA expression of Muc5AC, and Col1a1, normalized to Ppia. D, Representative western blots for $\alpha$-smooth muscle actin (SMA) levels and the loading control b-actin. $\mathbf{E}$, Assessment of SMA staining around large airways. Scale bar: $300 \mu \mathrm{m} . \mathrm{n}=5$ per group. ${ }^{*} \mathrm{P}<0.05 ;{ }^{* \star *} \mathrm{P}<$ 0.001 .

\section{Activation of PKM2 by TEPP46 attenuates IL-1 $\beta$-mediated pro-inflammatory responses in mouse lungs}

We have previously shown that increases in glycolysis promote pro-inflammatory responses in airway epithelial cells exposed to IL-1 $\beta$ by increasing the production of the proinflammatory cytokines TSLP, GM-CSF, KC and CCL20 (3). Results in Figure 1F demonstrate similar increases in IL-1 $\beta$ levels in lung tissue from HDM-exposed mice receiving vehicle or TEPP46, suggesting that TEPP46 does not regulate expression of IL-1 $\beta$. To examine whether PKM2 activity affects the responsiveness of lungs to IL-1 $\beta$, WT mice were intraperitoneally injected with TEPP46 prior to intranasal IL-1 $\beta$ instillation for either 6 or 24 hours (Figure 3A). TEPP46 increased the total PKM activity in WT mice, with no further increases being observed in response to IL-1 $\beta$ (Figure 3B) after 6 hours and similar results were observed after 24 hours (data not shown). As expected, IL-1 $\beta$ elicited increases in total cell counts, reflected by neutrophils 24 hours post intranasal administration, without affecting airway eosinophils, macrophages, and lymphocytes (Figure 3C and D). In animals receiving TEPP46, the IL-1 $\beta$-mediated increases in total cell counts and neutrophils were diminished (Figure $3 \mathrm{C}$ and $\mathrm{D}$ ). In agreement with these findings, mRNA expression levels of Csf2 and Ccl20 and the respective protein levels of GM-CSF, and CCL20, were significantly attenuated in mice treated with TEPP46 prior to IL-1 $\beta$ instillation for 6 hours (Figure 3E, F), while there was less to no effect on Cxcl1 and Ts/p mRNA and protein levels (KC and TSLP). Doses of $25 \mathrm{mg} / \mathrm{kg}$ or $50 \mathrm{mg} / \mathrm{kg}$ of TEPP46 were similar in their ability to induce PKM activity and dampen proinflammatory responses (Figure 3B, 3E, and 3F). Collectively, these results demonstrate that PKM2 activation decreases select IL-1 $\beta$-induced inflammatory responses in the lung. 
A
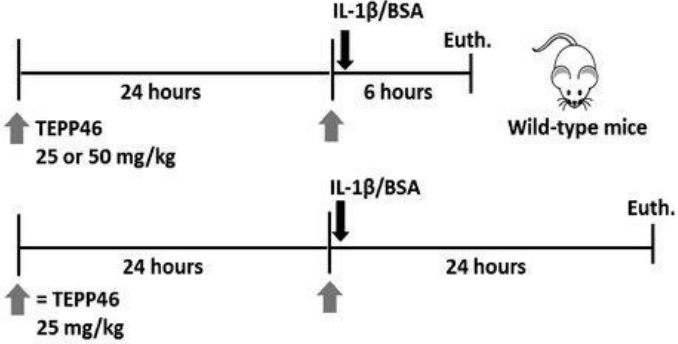

C
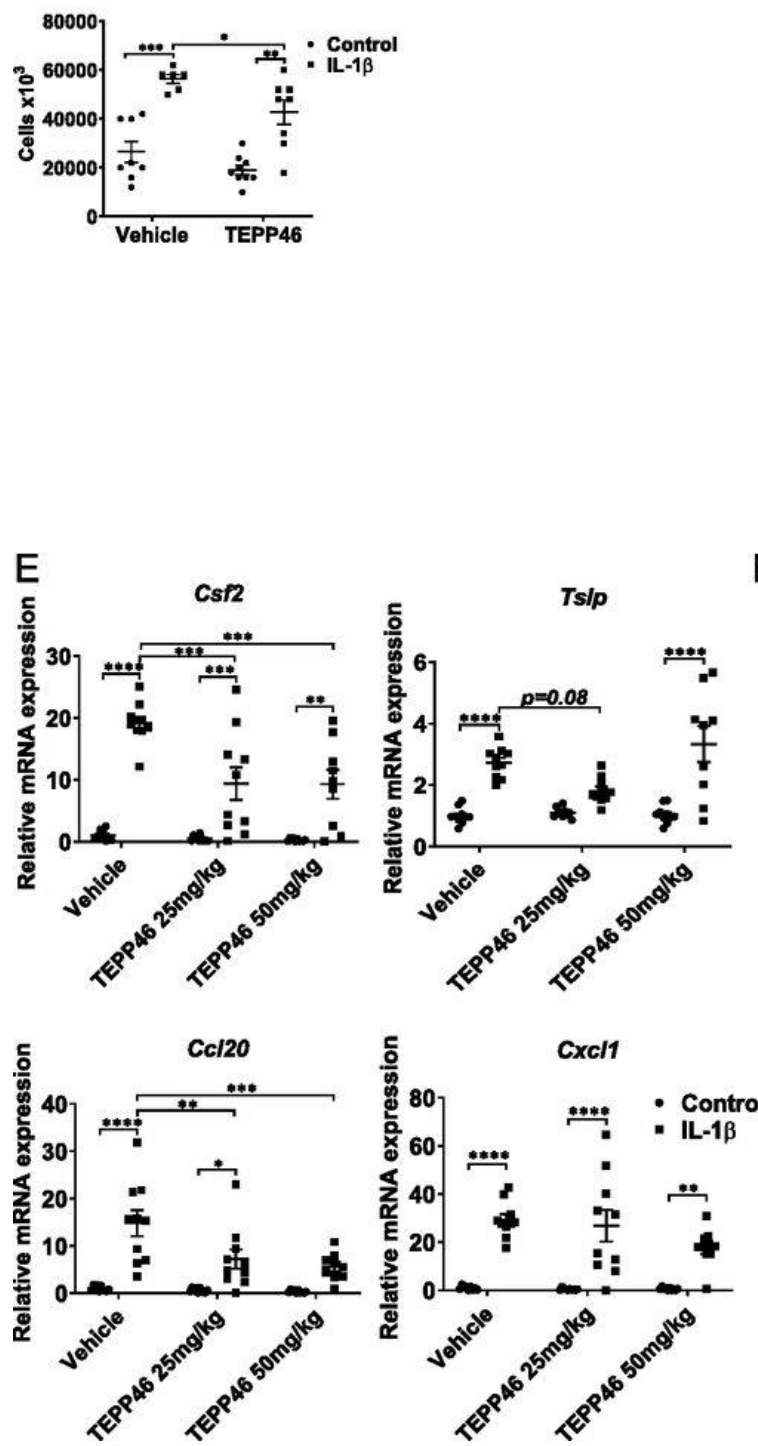

B PKM activity assay

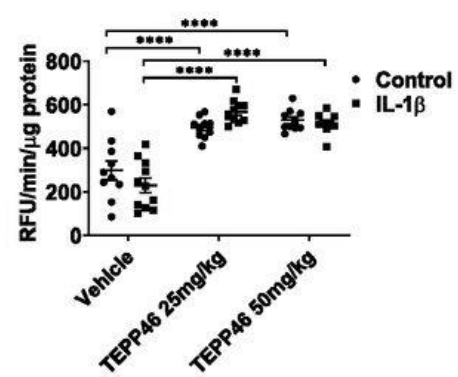

D
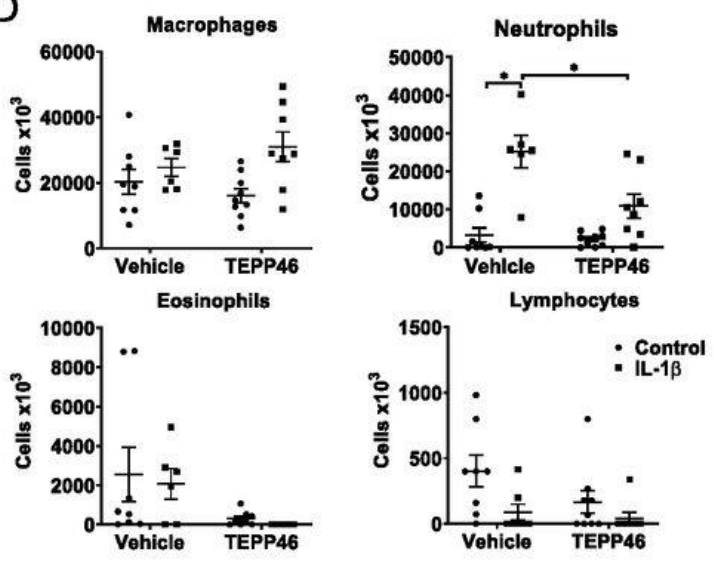

$\mathrm{F}$
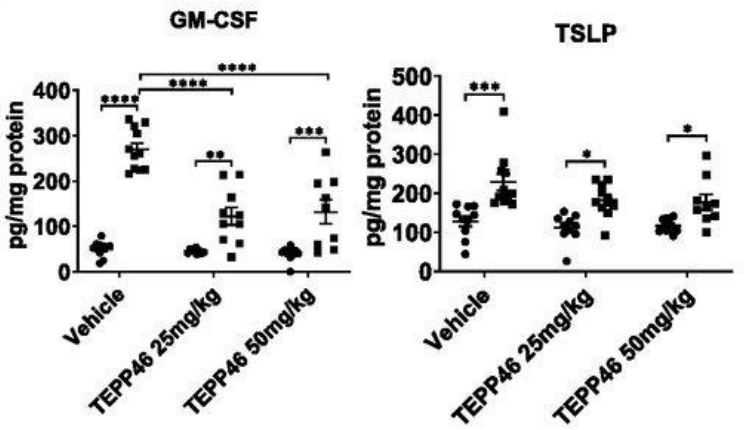

CCL20

KC
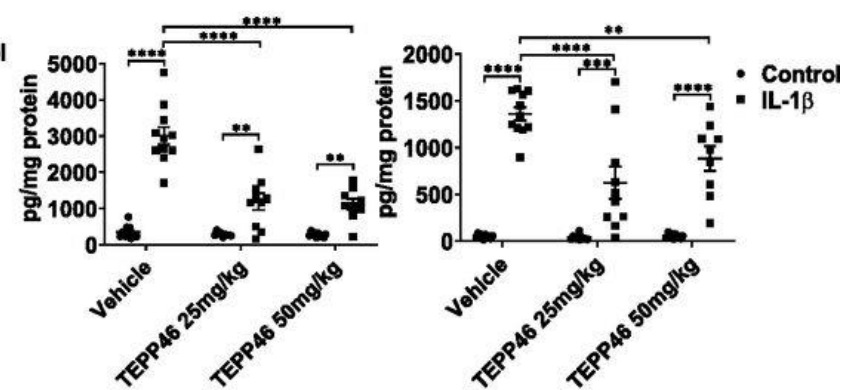

Figure 3: PKM2 activation attenuates the release of pro-inflammatory cytokines following intranasal administration of IL-1b. A, Schematic depicting the pre-treatment with 25 or $50 \mathrm{mg} / \mathrm{kg}$ TEPP46 prior to intranasal administration of $1 \mu \mathrm{g}$ of IL-1 $\beta$ for either 6 or 24 hours. The total cell count and cell differentials in the BAL fluid reflect 24 hours post IL-1 $\beta$ treatment, the other results shown are obtained 6 hours post IL$1 \beta$. $\mathbf{B}$, assessment of PKM activity in lung tissue homogenates. $\mathbf{C}$, and $\mathbf{D}$, Total and differential cell counts 
in BAL fluid. E, mRNA expression of pro-inflammatory cytokine genes in lung tissue homogenates. Results were normalized to the house keeping gene, Ppia. F, Levels of pro-inflammatory mediators TSLP, GMCSF, KC, and CCL20 in lung tissue homogenates by ELISA. $n=6-11$ per group. ${ }^{*} P<0.05$; ${ }^{* *} P<0.01$; ${ }^{* * *} P$ $<0.001 ;{ }^{* * *} \mathrm{P}<0.0001$.

\section{TEPP46 decreases nuclear translocation of PKM2 and dampens IL-1 $\beta$-mediated proinflammatory responses in mouse tracheal basal cells}

Airway epithelial cells are important contributory cells to allergic airway disease, as these cells release a number of mediators that promote innate and adaptive immune responses $(18,19)$. We previously demonstrated that IL-1 $\beta$-induced glycolysis is critical for the release of TSLP and other asthma-relevant cytokines by epithelial cells, and that IL-1 $\beta$-induced glycolysis also primes these cells to elicit augmented pro-inflammatory responses to HDM (3). PKM2 in its dimer form has a low binding affinity to PEP, and can translocate into the nucleus where it acts as a transcriptional co-activator to enhance transcription of multiple pro-inflammatory cytokines. We therefore next determined whether IL-1 $\beta$ affects the status and/or nuclear presence of PKM2 in epithelial cells, and whether PKM2 activation affects the response to IL-1 $\beta$. Primary MTE cells were pre-treated with TEPP46 for 1 hour, prior to IL-1 $\beta$ stimulation for 24 hours. This experimental regimen did not result in apparent changes in expression levels in PKM1 and PKM2 (Figure 4A). However, TEPP46 augmented overall PKM glycolytic activity in control cells, and a further enhancement of PKM activity occurred when cells were treated with TEPP46 in combination with IL-1 (Figure 4B). PKM2 is active as a glycolysis enzyme in its tetramer form, and loses its activity as a glycolytic kinase in the dimer form (11). Instead, dimeric PKM2 has been shown to translocate into the nucleus where it acts as a protein kinase to induce phosphorylation of STAT3, augmenting STAT3 transcriptional activity, leading to increased expression of proinflammatory mediators and increased expression of glycolysis enzymes including glucose transporter 1 (GLUT-1), thereby promoting glycolytic reprogramming (20). IL-1 $\beta$ led to a slight attenuation of PKM2 tetramers in MTE cells, while TEPP46 increased PKM2 tetramers in both control and IL-1 $\beta$-treated cells, relative to the respective vehicle groups (Figure $4 \mathrm{C}$ ). We did not observe an increase in nuclear PKM2 24 hrs post-administration of IL-1 3 . However, TEPP46 diminished the nuclear presence of PKM2 and increased its cytoplasmic localization, in both control and IL-1 $\beta$-stimulated cells (Figure 4D). Consistent with the attenuation of glycolytic reprogramming, TEPP46 administration led to a decrease in IL-1ß-mediated lactate secretion (Figure 4E). 
A

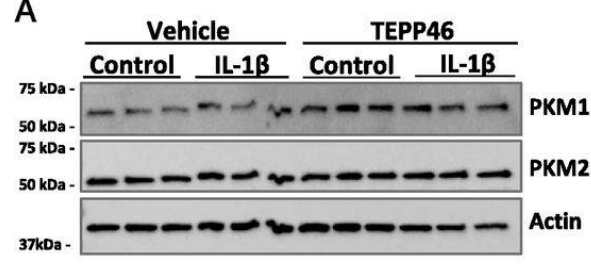

C Vehicle $\frac{\text { TEPP46 }}{\text { COnto }}$

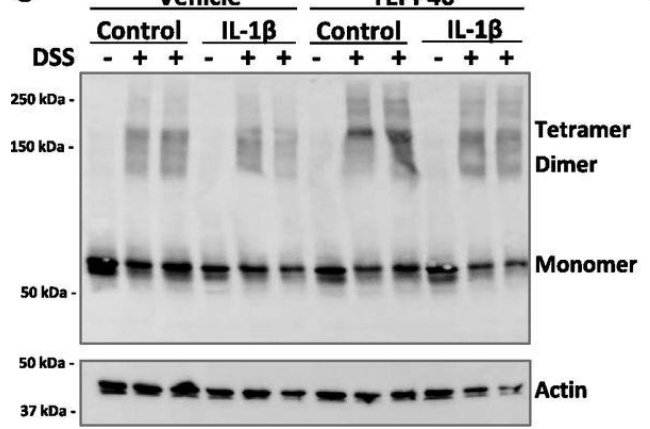

B

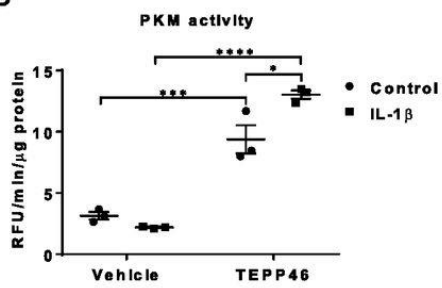

D

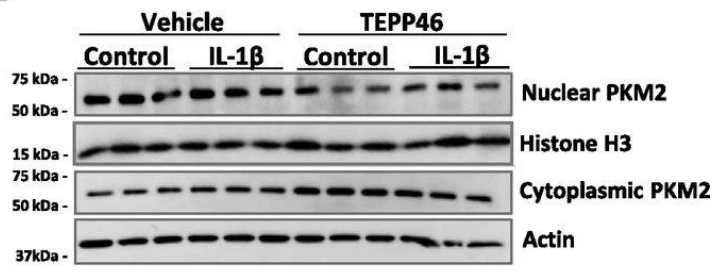

E

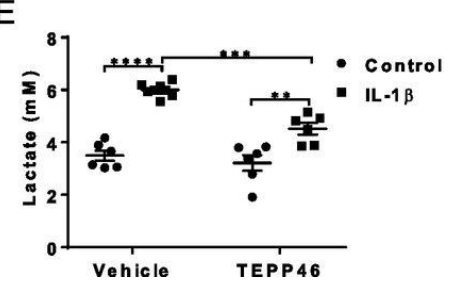

Figure 4: TEPP46 augments PKM activity, and PKM2's cytosolic presence and attenuates interleukin-1b-mediated lactate secretion in primary MTE cells. MTE cells were treated with $100 \mu \mathrm{M}$ TEPP46 for 1 hour prior to stimulation with $10 \mathrm{ng} / \mathrm{mL} \mathrm{IL-1 \beta}$ for 24 hours. A, representative western blot of total PKM1 and PKM2 levels, and b-actin. B, PKM activity assay in MTE cells and $\mathbf{C}$, representative western blot for tetrameric, dimeric and monomeric PKM2 and the loading control b-actin. MTE cells were incubated in the presence or absence (first lane of each condition) of the DSS crosslinker to evaluate the formation of the isoforms of PKM2. D, Representative western blots of nuclear and cytosolic extracts of PKM2. ( $n=3$ per group). E, Lactate levels in supernatants of MTE cells. Experiments were performed at least 3 times. ${ }^{*} \mathrm{P}<0.05 ;{ }^{* *} \mathrm{P}<0.01 ;{ }^{* *} \mathrm{P}<0.001 ;{ }^{* * *} \mathrm{P}<0.0001$.

To investigate whether activation of PKM2 attenuates pro-inflammatory cytokine release from airway epithelial cells, MTE cells were pre-treated with TEPP46 followed by stimulation with IL$1 \beta$ (Figure 5A). Strikingly, PKM2 activation strongly attenuated the IL-1 $\beta$-induced $m R N A$ and protein levels of Tslp (TSLP) and Csf2 (GM-CSF), respectively, while it modestly or did not affect Ccl20 (CCL20) or Cxcl1 (KC) (Figure 5B, C). As was stated earlier, exposure to IL-1 $\beta$ primes MTE cells to subsequent stimulation with HDM, leading to augmented release of pro-inflammatory cytokines. We therefore pre-treated primary MTE cells with TEPP46, followed by stimulation with 
IL-1 $\beta$ for 24 hours. Cells were then washed and exposed to HDM for 2 hours (Figure 5D). In agreement with our previous observations, prior exposure to IL-1 $\beta$ leads to potent HDMstimulated release of TSLP, GM-CSF, KC and CCL20. TEPP46 almost completely abolished TSLP and GM-CSF in this sequential exposure regimen, and significantly decreased KC and CCL20 (Figure 5E). TEPP46 treatment alone or in combination with IL-1 $\beta$ or IL-1 $\beta+H D M$ did not induce cell death (Figure $5 \mathrm{~F}$ ), demonstrating that the decreased cytokine production is not due to a loss of cell survival. Collectively, these data demonstrate that TEPP46 diminishes IL-1 $\beta$ and HDM-mediated proinflammatory responses in epithelial cells, in association with increases in PKM2 cytosolic presence and enhanced PKM glycolytic activity.

A

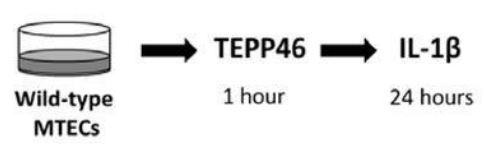

B

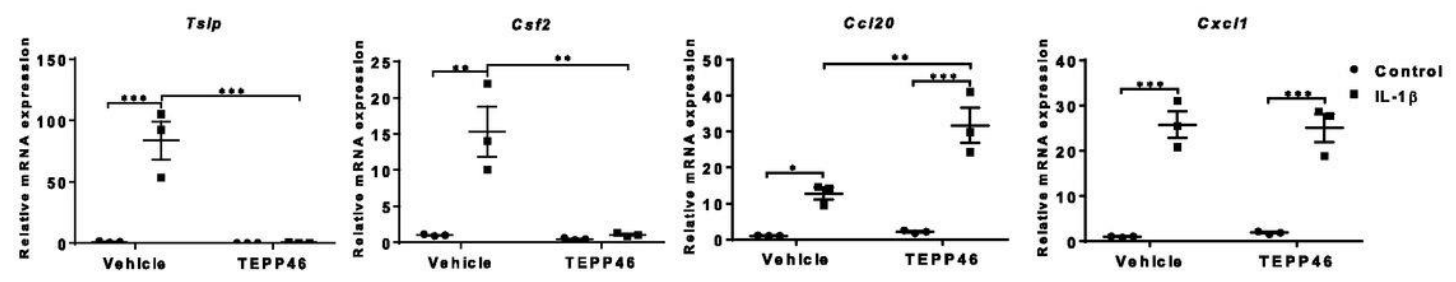

C
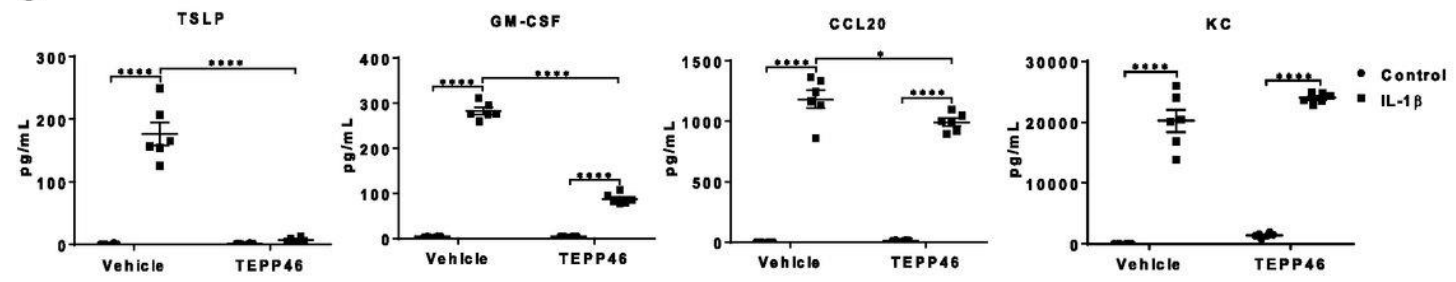

D
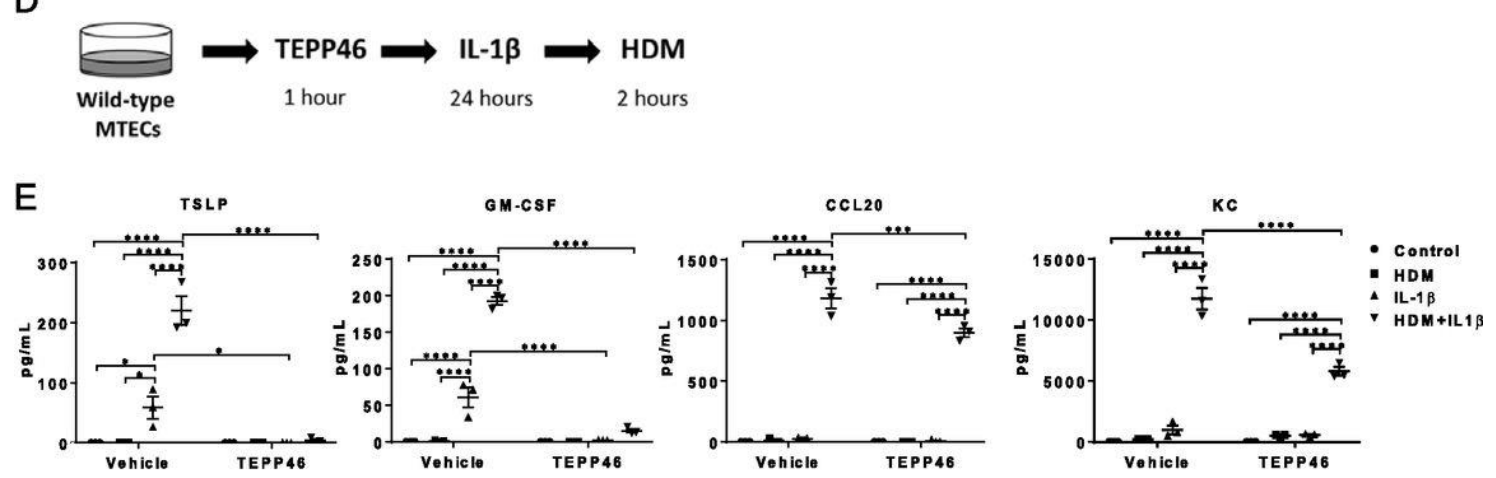

$\mathrm{F}$
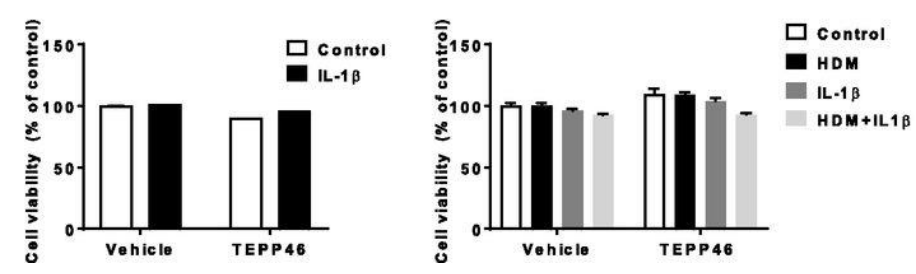
Figure 5: Activation of PKM2 attenuates IL-1b-mediated pro-inflammatory responses in primary MTE cells and the release of pro-inflammatory mediators following subsequent exposure to HDM. A, Schematic depicting the pre-treatment with $100 \mu \mathrm{M}$ TEPP46 followed by stimulation of $10 \mathrm{ng} / \mathrm{mL} \mathrm{IL}-1 \beta$ for 24 hours. B, mRNA expression of Ts/p, Csf2, Cxcl1 and Ccl20 in MTE cells. Ppia is used as housekeeping gene. C, Pro-inflammatory cytokine mediators TSLP, GM-CSF, KC, and CCL20 in cell culture supernatants of MTE cells were detected by ELISA. D, Schematic depicting the pre-treatment with $100 \mu \mathrm{M}$ TEPP46 followed by stimulation of $10 \mathrm{ng} / \mathrm{mL} \mathrm{IL}-1 \beta$ for 24 hours. Media was replaced and exposed to HDM (50 $\mu \mathrm{g} / \mathrm{mL}$ ) for an additional 2 hours. E, Proinflammatory cytokine mediators TSLP, GM-CSF, KC, and CCL20 in cell culture supernatants of MTE cells. F, Cell survival was evaluated by crystal violet staining (left) and Calcein AM assay (right) in MTE cells. $n=3-6$ per group. Experiments were performed at least 3 times. * $P$ $<0.05 ;{ }^{* \star} \mathrm{P}<0.01 ;{ }^{* \star *} \mathrm{P}<0.001 ;{ }^{* \star \star *} \mathrm{P}<0.0001$.

\section{PKM2-mediated phosphorylation of STAT3 contributes to IL-1 $\beta$-mediated proinflammatory signaling in epithelial cells}

It has been previously described that nuclear PKM2 phosphorylates STAT3, thereby augmenting the production of pro-inflammatory cytokines, including IL-6 and IL-1 $\beta(11,20)$. We therefore addressed whether PKM2 contributed to STAT3 activation, and in turn whether STAT3 promoted IL-1 $\beta$-induced pro-inflammatory signaling in mouse epithelial cells. Despite the lack of observed increases in nuclear PKM2 in response to IL-1 (Figure 4D), IL-1 $\beta$ elicited strong increases in nuclear pSTAT3 using an antibody directed against phosphorylation of tyrosine 705, the residue known to be phosphorylated by PKM2 (11) (Figure 6A). Total content of STAT3 in the nucleus was also increased in epithelial cells exposed to IL-1 $\beta$ (Figure 6A). Exposure to TEPP46 led to a strong diminution of nuclear pSTAT3 (Figure 6A), consistent with the aforementioned role of PKM2 as a STAT3 kinase (20). Similarly, phosphorylation of nuclear STAT3 was also increased in lung tissues from mice with HDM-induced allergic airways disease, and was diminished in mice also treated with TEPP46 (Figure 6B). We previously showed data suggesting that inhibitory kappa B kinase epsilon (IKKE) is a critical mediator in IL-1 $\beta$-induced glycolysis. Here we show that the IL-1 $\beta$-induced expression levels of IKKE were unaffected when MTE cells were pretreated with TEPP46, suggesting that the effect of TEPP46 on diminishing STAT3 phosphorylation may be downstream or independent of IKKE (Figure 6A). To further corroborate the role of STAT3 in promoting IL-1 $\beta$-induced pro-inflammatory responses, we used the STAT3 inhibitor, Stattic (17, 20 ), in vitro. Concentrations greater than $1 \mu \mathrm{M}$ Stattic caused marked epithelial cell death (Figure $6 \mathrm{C})$. Nonetheless, a concentration of $0.5 \mu \mathrm{M}$ Stattic diminished IL-1 $\beta$-mediated phosphorylation of STAT3 in whole cell lysates (Figure 


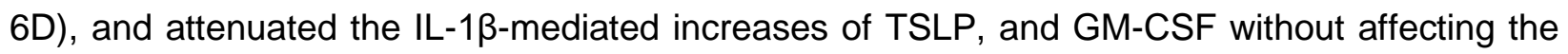
other cytokines (Figure 6E). All together, these data suggest that activation of PKM2 as a glycolytic kinase by TEPP46 diminishes the pro-inflammatory responses induced by IL-1 $\beta$ in lung epithelial cells or in mice with allergic airway disease, and that the increased kinase activity of PKM2 towards STAT3 in these settings may in part contribute to PKM2-linked inflammation.
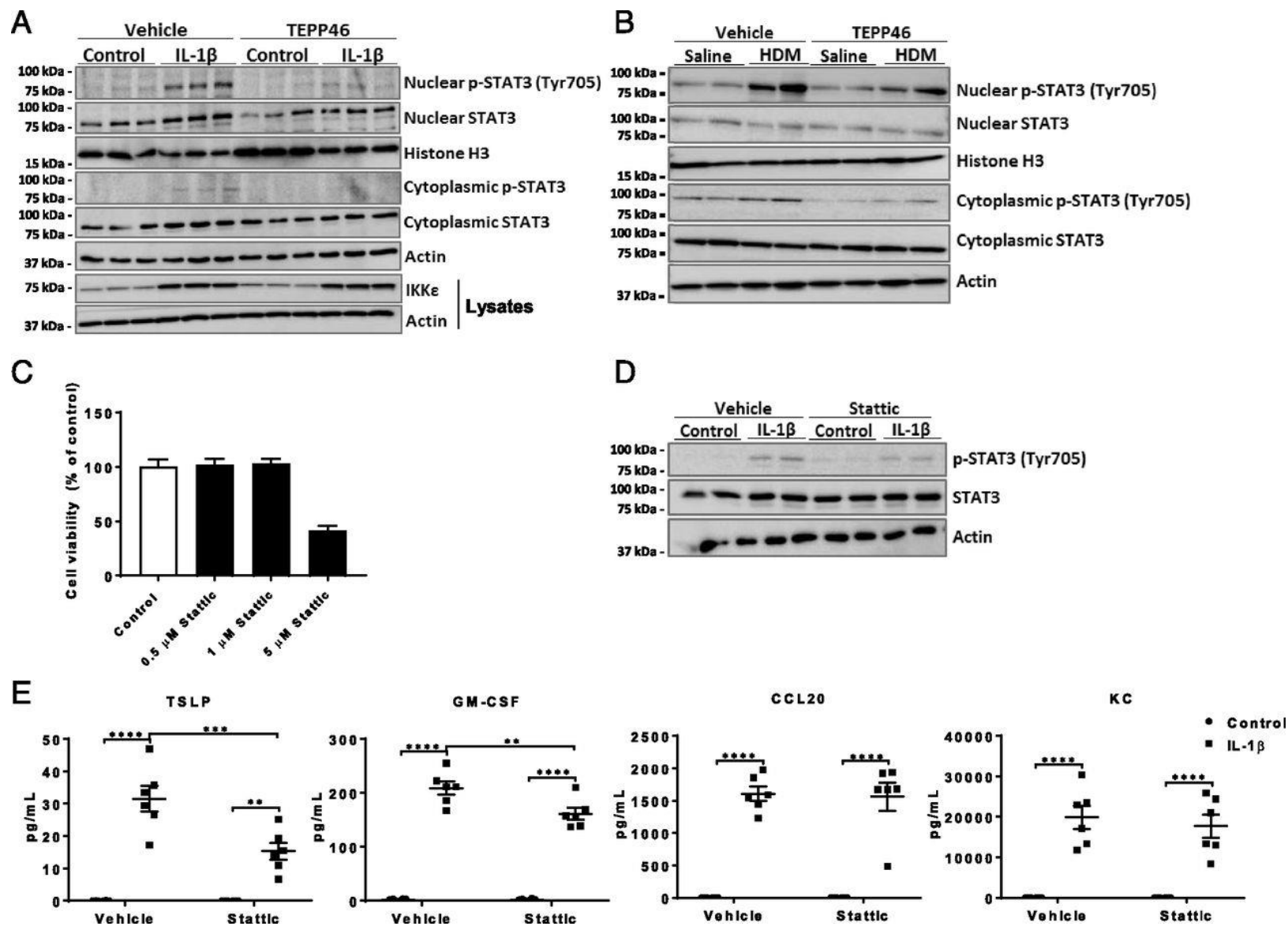

Figure 6: PKM2-mediated phosphorylation of STAT3 contributes to IL-1b-mediated proinflammatory signaling in epithelial cells. A, Representative western blots of total and phosphorylated STAT3 in nuclear and cytosolic extracts from MTE cells, and total IKKe levels in whole cell lysates. B, Representative western blots of total and phosphorylated STAT3 in nuclear and cytosolic extracts from HDM- or saline-treated lung tissues. C, Impact of Stattic on survival of MTE cells was evaluated by a Calcein AM assay. D, Representative western blots of total and phosphorylated STAT3 in whole cell lysates from control or IL-1 $\beta$ stimulated MTE cells pre-treated with Stattic or vehicle control. E, Pro-inflammatory mediators TSLP, GM-CSF, KC, and CCL20 in cell culture supernatants of MTE cells, after treatment for 1 hour with $0.5 \mu \mathrm{M}$ Stattic, followed by stimulation of $10 \mathrm{ng} / \mathrm{mL} \mathrm{IL}-1 \beta$ for 24 hours. Experiments were conducted at least 3 times. ${ }^{* *} \mathrm{P}<0.01 ;{ }^{* \star *} \mathrm{P}<0.001 ;{ }^{* * *} \mathrm{P}<0.0001$. 


\section{DISCUSSION}

Allergic airway disease is associated with chronic inflammation and airway remodeling, processes that are metabolically demanding. During glycolysis, some of the carbons derived from glucose are used to allow for biosynthetic processes. In addition, glycolysis has also been linked to proinflammatory responses in immune cells $(21,22)$. Our laboratory has previously shown that glycolysis is a feature of allergic asthma in association with neutrophilic inflammation and steroidresistant disease, and that IL1 is an important driver of glycolysis in settings of allergic airways disease in mice (3). In addition to these observations, increases in aerobic glycolysis have been shown to promote $\mathrm{T}$ cell activation (23) and to promote $\mathrm{T}$ cell effector function (21). Increases in glycolysis also have been implicated in lipopolysaccharide (LPS)-induced airway smooth muscle cell proliferation (24) and in IL-33-mediated increases in cytokine production in mast cells (25). Asthma associated single-nucleotide polymorphisms within the orosomucoid-like 3 (ORMDL3) locus have been implicated in disease susceptibility. A recent study showed that ablation of ORMDL3 attenuated IL1-mediated endoplasmic reticulum stress and cytokine responses in A549 lung epithelial cells, in association with alterations in glycolysis and glucose metabolism genes (26) indicating a potential link between glycolysis and asthma susceptibility. Increases in glycolysis, basal and maximal respiration, and oxidative stress were demonstrated in airway epithelial cells and platelets from obese asthmatics (who tend to have more severe disease), in comparison to lean asthmatics and healthy subjects (27). Notably, increases in airway lactate were demonstrated in asthmatics with a high fraction of exhaled nitric oxide (FeNO), in association with elevated expression of inducible nitric oxide synthase and arginase 2, and suggests a link between enhanced glycolysis, arginine metabolism and a high FeNO asthma phenotype (28). Nonetheless, the precise signals and settings that elicit the glycolysis-associated proinflammatory responses in lung epithelial cells remain unclear. In the present study we demonstrate the importance of the glycolytic enzyme PKM2 in promoting inflammation and airway remodeling in mice with HDM induced allergic airway disease. PKM2 has generated substantial interest due to its impact on glycolytic reprogramming in activated immune cells and tumor cells, and its emerging role as a pro-inflammatory mediator $(9,12)$. Herein we demonstrate that activation of the glycolysis function of PKM2 with TEPP46, augments pyruvate kinase activity in lung tissue and airway epithelial cells, and dampens inflammation, evidenced by attenuated airway eosinophilia and airway remodeling in mice with HDM-induced allergic airways disease. Moreover, administration of TEPP46 attenuated IL-1 $\beta$-induced airway neutrophilia in mice and significantly reduced IL-1 $\beta$-mediated expression of pro-inflammatory cytokines and lactate. These results, in addition to our previous results (3), show that enhanced glycolysis is important for the 
amplification of allergen-induced pro-inflammatory responses and show the importance that PKM2 plays in regulating this process.

Results herein point to the importance of glycolysis in the secretion of specific proinflammatory mediators from airway epithelial cells, notably TSLP and GM-CSF. Activation of PKM2 with TEPP46 almost completely abolished expression of both cytokines, while Stattic also attenuated the IL-1 $\beta$-mediated release of these cytokines (Figure 5C, and 6E). These findings are in line with our previous study wherein we demonstrated that inhibition of inhibitory kappa $B$ kinase epsilon (IKBKE) or TANK-binding kinase 1 (TBK1), two kinases critical in promoting IL-1 $\beta$-induced glycolysis also virtually abolished secretion of TSLP and GM-CSF (3). The importance of TSLP in asthma has been extensively studied $(22,29-32)$. TSLP is primarily expressed in epithelial cells and acts on both innate and adaptive immune cells thereby promoting T-helper 2 immunity and steroid resistance (33). Overexpression of TSLP results in the development of severe airway inflammation and airway hyper responsiveness $(29,32)$. The USA Food and Drug Administration (FDA) has granted Breakthrough Therapy Designation for tezepelumab, a TSLP-blocker, in patients with severe asthma. Blocking TSLP may prevent the release of other pro-inflammatory cytokines by immune cells resulting in the prevention of asthma exacerbations and improved asthma control (34). Similarly, GM-CSF has been shown to activate macrophages and promote eosinophil migration, differentiation and survival, in addition to its function in the differentiation and maturation of dendritic cells (35). Our present data showing that activation of PKM2 with TEPP46, preferentially attenuates TSLP and GM-CSF, while modestly or not affecting CCL20 and $\mathrm{KC}$, suggest that avenues to attenuate glycolysis, or to activate PKM2 in an environment where IL-1 signaling is operative will be attractive strategies to dampen TSLP and GM-CSF.

The pro-inflammatory role of PKM2 has been extensively studied in tumor and immune cells, and a number of transcription factors have been demonstrated to mediate the proinflammatory effect of PKM2 (36-38). In macrophages and tumor cells, LPS induces nuclear binding of PKM2 to hypoxia-inducible factor 1-alpha (HIF-1a) $(20,39)$. In tumor cells, an interaction between PKM2 and Jumonji C domain-containing dioxygenase (JMJD5) also has been shown (37). These interactions with PKM2 have been linked to the transcription of glycolysis genes including LDHA and GLUT-1. Moreover, nuclear PKM2 was also shown to phosphorylate STAT3 $(13,36)$, which in turn augments IL-1 $\beta$ and IL-6 production (20). In line with the latter findings, the present observations that IL-1 $\beta$ or HDM led to increases in phosphorylation of STAT3, which were attenuated by TEPP46, suggest the importance of PKM2 in promoting STAT3 phosphorylation. Our findings that inhibition of STAT3 attenuated release of TSLP and GM-CSF in airway basal 
cells, strongly suggest that the ability of PKM2 to phosphorylate STAT3 contributes to its proinflammatory function. Findings from the present study using an activator of PKM2 are in line with another study showing that activation of the glycolysis function of PKM2 attenuated the proinflammatory phenotype of macrophages from patients with atherosclerotic coronary artery disease (20), and inhibited the PKM2-HIF-1a complex (39) leading to decreased IL-1 $1 \beta$ production and glycolysis and pro-inflammatory genes $(38,39)$. Moreover, it has been shown that inhibition of STAT3, by Stattic, attenuated inflammatory injury in LPS-challenged mice (14). PKM2 and PKM1 are both encoded by the same PKM gene, however they represent different splicing products (exon 9 for PKM1, exon 10 for PKM2). Unlike PKM1, PKM2 is not a constitutive stable tetrameric enzyme, and can be allosterically regulated by fructose-1,6- bisphosphate (FBP) to enhance tetramer formation. The PKM2 tetramer can be converted to dimers following a number of post translational modifications that include phosphorylation $(40,41)$, acetylation $(42,43)$, oxidation (44), hydroxylation (37, 38), ubiquitination (45), glycosylation (46), methylation (47), and sumoylation (48) in response to various stimuli. Interestingly, epidermal growth factor (EGF)activated ERK2 binds directly to PKM2 and can induce phosphorylation of PKM2 at serine 37, in association with its nuclear translocation and increases in transcriptional activation of GLUT-1, and LDHA $(49,50)$. The EGF receptor (EGFR) is of notable interest due to its role of type 2 inflammatory responses in allergic airways disease, including mucus metaplasia (51-53). Additional studies will be required to elucidate whether EGFR activation contributed to phosphorylation and subsequently the inactivation of PKM2 that was observed in the present study. Other studies have demonstrated that PKM2 can be inactivated following oxidation of cysteine 358 (44). Changes in the oxidative environment and notably cysteine oxidations accompany allergic airway disease and lead to activation of EGFR in cells (51). Similarly, changes in the oxidative environment also control dendritic cell activation and Tcell subsets (54). Additional studies will also be required to address whether oxidative events regulate PKM2 activity herein.

Small molecule activators of PKM2 such as TEPP46 (also known as ML265) and DASA-58 have been developed to stabilize PKM2 in the tetramer configuration. TEPP46 activates PKM2 by binding to the dimer-dimer interface between two subunits of PKM2, which stabilizes tetrameric PKM2 to promote conversion of PEP to pyruvate, hence increasing its glycolytic activity. TEPP46 is highly selective in its ability to activate PKM2 (55), since it does not affect recombinant PKM1 in vitro (10) and has no significant effect in PKM2 knockout models $(39,56)$. These observations suggest that the effects observed by TEPP46 herein are due to the activation of the glycolysis function of PKM2, and not due to off target effects, although additional studies will be required to corroborate the lack of off target effects. Paradoxically, while activation of PKM2 dampened 
inflammation, ablation of PKM2 also elicited anti-inflammatory effects. As discussed earlier, the TEPP46-induced PKM2 tetramer inhibited LPS-induced expression of IL-1 $\beta$ and other HIF-1 $\alpha$ dependent genes in macrophages $(20,39)$, while macrophages lacking PKM2 also showed reduced expression of $I L-1 \beta$ and $L d$ ha mRNAs in response to LPS. These findings suggest that dimeric PKM2 has a pro-inflammatory gain of function, and that strategies to either remove PKM2 altogether, or to promote its glycolysis kinase function elicit similar anti-inflammatory effects. Another limitation of the current manuscript is that experiments were performed in mice only.

Further studies using human samples will be required to fully understand the contribution of PKM2 to pro-inflammatory responses in epithelial cells or airways from asthmatics. Altogether, our results demonstrate that the glycolysis-inactive form of PKM2 plays a crucial role in the pathogenesis of allergic airway disease in association with enhancing IL- $1 \beta$-induced proinflammatory signaling, in part through phosphorylation of STAT3, and notably the upregulation of $T s / p$ and Csf2 genes. PKM2 therefore could be a novel potential target for the development of anti-inflammatory therapies for the treatment of IL1 high, glycolysis-associated asthma. 


\section{REFERENCES}

1. Fahy, J. V. 2015. Type 2 inflammation in asthma--present in most, absent in many. Nature reviews. Immunology 15: 57-65.

2. Domblides, C., L. Lartigue, and B. Faustin. 2018. Metabolic Stress in the Immune Function of T Cells, Macrophages and Dendritic Cells. Cells 7.

3. Qian, X., R. Aboushousha, C. van de Wetering, S. B. Chia, E. Amiel, R. W. Schneider, J. L. J. van der Velden, K. G. Lahue, D. A. Hoagland, D. T. Casey, N. Daphtary, J. L. Ather, M. J. Randall, M. Aliyeva, K. E. Black, D. G. Chapman, L. K. A. Lundblad, D. H. McMillan, A. E. Dixon, V. Anathy, C. G. Irvin, M. E. Poynter, E. F. M. Wouters, P. M. Vacek, M. Henket, F. Schleich, R. Louis, A. van der Vliet, and Y. M. W. Janssen-Heininger. 2018. IL-1/inhibitory kappaB kinase epsilon-induced glycolysis augment epithelial effector function and promote allergic airways disease. The Journal of allergy and clinical immunology 142: 435-450.e410.

4. Doherty, J. R., and J. L. Cleveland. 2013. Targeting lactate metabolism for cancer therapeutics. The Journal of clinical investigation 123: 3685-3692.

5. Ward, P. S., and C. B. Thompson. 2012. Metabolic reprogramming: a cancer hallmark even warburg did not anticipate. Cancer cell 21: 297-308.

6. Noguchi, T., H. Inoue, and T. Tanaka. 1986. The M1- and M2-type isozymes of rat pyruvate kinase are produced from the same gene by alternative RNA splicing. The Journal of biological chemistry 261: 1380713812.

7. Noguchi, T., K. Yamada, H. Inoue, T. Matsuda, and T. Tanaka. 1987. The L- and R-type isozymes of rat pyruvate kinase are produced from a single gene by use of different promoters. The Journal of biological chemistry 262: 14366-14371.

8. Mazurek, S. 2011. Pyruvate kinase type M2: a key regulator of the metabolic budget system in tumor cells. The international journal of biochemistry \& cell biology 43: 969-980.

9. Christofk, H. R., M. G. Vander Heiden, M. H. Harris, A. Ramanathan, R. E. Gerszten, R. Wei, M. D. Fleming, S. L. Schreiber, and L. C. Cantley. 2008. The M2 splice isoform of pyruvate kinase is important for cancer metabolism and tumour growth. Nature 452: 230-233.

10. Anastasiou, D., Y. Yu, W. J. Israelsen, J. K. Jiang, M. B. Boxer, B. S. Hong, W. Tempel, S. Dimov, M. Shen, A. Jha, H. Yang, K. R. Mattaini, C. M. Metallo, B. P. Fiske, K. D. Courtney, S. Malstrom, T. M. Khan, C. Kung, A. P. Skoumbourdis, H. Veith, N. Southall, M. J. Walsh, K. R. Brimacombe, W. Leister, S. Y. Lunt, Z. R. Johnson, K. E. Yen, K. Kunii, S. M. Davidson, H. R. Christofk, C. P. Austin, J. Inglese, M. H. Harris, J. M. Asara, G. Stephanopoulos, F. G. Salituro, S. Jin, L. Dang, D. S. Auld, H. W. Park, L. C. Cantley, C. J. Thomas, and M. G. Vander Heiden. 2012. Pyruvate kinase M2 activators promote tetramer formation and suppress tumorigenesis. Nature chemical biology 8: 839- 847.

11. Gao, X., H. Wang, J. J. Yang, X. Liu, and Z. R. Liu. 2012. Pyruvate kinase M2 regulates gene transcription by acting as a protein kinase. Molecular cell 45: 598-609.

12. Alves-Filho, J. C., and E. M. Palsson-McDermott. 2016. Pyruvate Kinase M2: A Potential Target for Regulating Inflammation. Frontiers in immunology 7: 145.

13. Demaria, M., and V. Poli. 2012. PKM2, STAT3 and HIF-1alpha: The Warburg's vicious circle. Jak-stat 1: 194-196. 
Activation of PKM2 attenuates pro-inflammatory mediators in HDM-induced allergic airways disease

14. Hu, K., Y. Yang, L. Lin, Q. Ai, J. Dai, K. Fan, P. Ge, R. Jiang, J. Wan, and L. Zhang. 2018. Caloric Restriction Mimetic 2-Deoxyglucose Alleviated Inflammatory Lung Injury via Suppressing Nuclear Pyruvate Kinase M2Signal Transducer and Activator of Transcription 3 Pathway. Frontiers in immunology 9: 426.

15. Wu, R., and D. Smith. 1982. Continuous multiplication of rabbit tracheal epithelial cells in a defined, hormone supplemented medium. In Vitro 18: 800-812.

16. Alcorn, J. F., A. S. Guala, J. van der Velden, B. McElhinney, C. G. Irvin, R. J. Davis, and Y. M. JanssenHeininger. 2008. Jun N-terminal kinase 1 regulates epithelial-to-mesenchymal transition induced by TGFbeta1. J Cell Sci 121: 1036-1045.

17. McMurray, J. S. 2006. A new small-molecule Stat3 inhibitor. Chemistry \& biology 13: 1123-1124.

18. Holtzman, M. J., D. E. Byers, J. Alexander-Brett, and X. Wang. 2014. The role of airway epithelial cells and innate immune cells in chronic respiratory disease. Nature reviews immunology 14: 686-698.

19. Hahn, C., A. P. Islamian, H. Renz, and W. A. Nockher. 2006. Airway epithelial cells produce neurotrophins and promote the survival of eosinophils during allergic airway inflammation. The Journal of allergy and clinical immunology 117: 787-794.

20. Shirai, T., R. R. Nazarewicz, B. B. Wallis, R. E. Yanes, R. Watanabe, M. Hilhorst, L. Tian, D. G. Harrison, J. C. Giacomini, T. L. Assimes, J. J. Goronzy, and C. M. Weyand. 2016. The glycolytic enzyme PKM2 bridges metabolic and inflammatory dysfunction in coronary artery disease. J Exp Med 213: 337-354.

21. Michalek, R. D., V. A. Gerriets, S. R. Jacobs, A. N. Macintyre, N. J. Maclver, E. F. Mason, S. A. Sullivan, A. G. Nichols, and J. C. Rathmell. 2011. Cutting edge: distinct glycolytic and lipid oxidative metabolic programs are essential for effector and regulatory CD4+ T cell subsets. Journal of immunology (Baltimore, Md. : 1950) 186: 3299-3303.

22. Lambrecht, B. N., H. Hammad, and J. V. Fahy. 2019. The Cytokines of Asthma. Immunity 50: 975-991.

23. Ostroukhova, M., N. Goplen, M. Z. Karim, L. Michalec, L. Guo, Q. Liang, and R. Alam. 2012. The role of low-level lactate production in airway inflammation in asthma. American journal of physiology. Lung cellular and molecular physiology 302: L300-307.

24. Zhang, L., C. Ma, X. Wang, S. He, Q. Li, Y. Zhou, Y. Liu, M. Zhang, X. Yu, X. Zhao, F. Li, and D. L. Zhu. 2019. Lipopolysaccharide-induced proliferation and glycolysis in airway smooth muscle cells via activation of Drp1. Journal of cellular physiology 234: 9255-9263.

25. Caslin, H. L., M. T. Taruselli, T. Haque, N. Pondicherry, E. A. Baldwin, B. O. Barnstein, and J. J. Ryan. 2018. Inhibiting Glycolysis and ATP Production Attenuates IL-33-Mediated Mast Cell Function and Peritonitis. Frontiers in immunology 9: 3026.

26. Zhang, Y., S. A. G. Willis-Owen, S. Spiegel, C. M. Lloyd, M. F. Moffatt, and W. Cookson. 2019. The ORMDL3 Asthma Gene Regulates ICAM1 and Has Multiple Effects on Cellular Inflammation. Am J Respir Crit Care Med 199:478-488.

27. Winnica, D., C. Corey, S. Mullett, M. Reynolds, G. Hill, S. Wendell, L. Que, F. Holguin, and S. Shiva. 2019. Bioenergetic Differences in the Airway Epithelium of Lean Versus Obese Asthmatics Are Driven by Nitric Oxide and Reflected in Circulating Platelets. Antioxidants \& redox signaling.

28. Xu, W., S. A. A. Comhair, A. J. Janocha, A. Lara, L. A. Mavrakis, C. D. Bennett, S. C. Kalhan, and S. C. Erzurum. 2017. Arginine metabolic endotypes related to asthma severity. PLoS One 12: e0183066. 
29. West, E. E., M. Kashyap, and W. J. Leonard. 2012. TSLP: A Key Regulator of Asthma Pathogenesis. Drug discovery today. Disease mechanisms 9.

30. Smelter, D. F., V. Sathish, M. A. Thompson, C. M. Pabelick, R. Vassallo, and Y. S. Prakash. 2010. Thymic stromal lymphopoietin in cigarette smoke-exposed human airway smooth muscle. Journal of immunology (Baltimore, Md.: 1950) 185: 3035-3040.

31. Demehri, S., M. Morimoto, M. J. Holtzman, and R. Kopan. 2009. Skin-derived TSLP triggers progression from epidermal-barrier defects to asthma. PLoS Biol 7: e1000067.

32. Zhou, B., M. B. Headley, T. Aye, J. Tocker, M. R. Comeau, and S. F. Ziegler. 2008. Reversal of thymic stromal lymphopoietin-induced airway inflammation through inhibition of Th2 responses. Journal of immunology (Baltimore, Md. : 1950) 181: 6557-6562.

33. Ziegler, S. F., F. Roan, B. D. Bell, T. A. Stoklasek, M. Kitajima, and H. Han. 2013. The biology of thymic stromal lymphopoietin (TSLP). Advances in pharmacology (San Diego, Calif.) 66: 129-155.

34. Gauvreau, G. M., P. M. O'Byrne, L. P. Boulet, Y. Wang, D. Cockcroft, J. Bigler, J. M. FitzGerald, M. Boedigheimer, B. E. Davis, C. Dias, K. S. Gorski, L. Smith, E. Bautista, M. R. Comeau, R. Leigh, and J. R. Parnes. 2014. Effects of an anti-TSLP antibody on allergen-induced asthmatic responses. The New England journal of medicine 370: 2102-2110.

35. Shi, Y., C. H. Liu, A. I. Roberts, J. Das, G. Xu, G. Ren, Y. Zhang, L. Zhang, Z. R. Yuan, H. S. Tan, G. Das, and S. Devadas. 2006. Granulocyte-macrophage colony-stimulating factor (GM-CSF) and T-cell responses: what we do and don't know. Cell research 16: 126-133.

36. Yang, P., Z. Li, H. Li, Y. Lu, H. Wu, and Z. Li. 2015. Pyruvate kinase M2 accelerates pro-inflammatory cytokine secretion and cell proliferation induced by lipopolysaccharide in colorectal cancer. Cellular signalling 27: 1525-1532.

37. Wang, H. J., Y. J. Hsieh, W. C. Cheng, C. P. Lin, Y. S. Lin, S. F. Yang, C. C. Chen, Y. Izumiya, J. S. Yu, H. J. Kung, and W. C. Wang. 2014. JMJD5 regulates PKM2 nuclear translocation and reprograms HIF-1alphamediated glucose metabolism. Proceedings of the National Academy of Sciences of the United States of America 111: 279-284.

38. Luo, W., H. Hu, R. Chang, J. Zhong, M. Knabel, R. O'Meally, R. N. Cole, A. Pandey, and G. L. Semenza. 2011. Pyruvate kinase M2 is a PHD3-stimulated coactivator for hypoxia-inducible factor 1. Cell 145: 732744.

39. Palsson-McDermott, E. M., A. M. Curtis, G. Goel, M. A. Lauterbach, F. J. Sheedy, L. E. Gleeson, M. W. van den Bosch, S. R. Quinn, R. Domingo-Fernandez, D. G. Johnston, J. K. Jiang, W. J. Israelsen, J. Keane, C. Thomas, C. Clish, M. Vander Heiden, R. J. Xavier, and L. A. O'Neill. 2015. Pyruvate kinase M2 regulates Hif1alpha activity and IL-1beta induction and is a critical determinant of the warburg effect in LPS-activated macrophages. Cell Metab 21:65-80.

40. Hitosugi, T., S. Kang, M. G. Vander Heiden, T. W. Chung, S. Elf, K. Lythgoe, S. Dong, S. Lonial, X. Wang, G. Z. Chen, J. Xie, T. L. Gu, R. D. Polakiewicz, J. L. Roesel, T. J. Boggon, F. R. Khuri, D. G. Gilliland, L. C. Cantley, J. Kaufman, and J. Chen. 2009. Tyrosine phosphorylation inhibits PKM2 to promote the Warburg effect and tumor growth. Science signaling 2: ra73.

41. Christofk, H. R., M. G. Vander Heiden, N. Wu, J. M. Asara, and L. C. Cantley. 2008. Pyruvate kinase M2 is a phosphotyrosine-binding protein. Nature 452: 181-186. 
42. Lv, L., D. Li, D. Zhao, R. Lin, Y. Chu, H. Zhang, Z. Zha, Y. Liu, Z. Li, Y. Xu, G. Wang, Y. Huang, Y. Xiong, K. L. Guan, and Q. Y. Lei. 2011. Acetylation targets the $M 2$ isoform of pyruvate kinase for degradation through chaperone mediated autophagy and promotes tumor growth. Molecular cell 42: 719-730.

43. Bhardwaj, A., and S. Das. 2016. SIRT6 deacetylates PKM2 to suppress its nuclear localization and oncogenic functions. Proceedings of the National Academy of Sciences of the United States of America 113: E538-547.

44. Anastasiou, D., G. Poulogiannis, J. M. Asara, M. B. Boxer, J. K. Jiang, M. Shen, G. Bellinger, A. T. Sasaki, J. W. Locasale, D. S. Auld, C. J. Thomas, M. G. Vander Heiden, and L. C. Cantley. 2011. Inhibition of pyruvate kinase M2 by reactive oxygen species contributes to cellular antioxidant responses. Science 334: 12781283.

45. Liu, K., F. Li, H. Han, Y. Chen, Z. Mao, J. Luo, Y. Zhao, B. Zheng, W. Gu, and W. Zhao. 2016. Parkin Regulates the Activity of Pyruvate Kinase M2. The Journal of biological chemistry 291: 10307-10317.

46. Wang, Y., J. Liu, X. Jin, D. Zhang, D. Li, F. Hao, Y. Feng, S. Gu, F. Meng, M. Tian, Y. Zheng, L. Xin, X. Zhang, X. Han, L. Aravind, and M. Wei. 2017. O-GlcNAcylation destabilizes the active tetrameric PKM2 to promote the Warburg effect. Proceedings of the National Academy of Sciences of the United States of America 114: 13732-13737.

47. Liu, F., F. Ma, Y. Wang, L. Hao, H. Zeng, C. Jia, Y. Wang, P. Liu, I. M. Ong, B. Li, G. Chen, J. Jiang, S. Gong, L. Li, and W. Xu. 2017. PKM2 methylation by CARM1 activates aerobic glycolysis to promote tumorigenesis. Nature cell biology 19: 1358-1370.

48. Spoden, G. A., D. Morandell, D. Ehehalt, M. Fiedler, P. Jansen-Durr, M. Hermann, and W. Zwerschke. 2009. The SUMO-E3 ligase PIAS3 targets pyruvate kinase M2. Journal of cellular biochemistry 107: 293302.

49. Yang, W., Y. Xia, H. Ji, Y. Zheng, J. Liang, W. Huang, X. Gao, K. Aldape, and Z. Lu. 2011. Nuclear PKM2 regulates beta-catenin transactivation upon EGFR activation. Nature 480: 118-122.

50. Yang, W., Y. Zheng, Y. Xia, H. Ji, X. Chen, F. Guo, C. A. Lyssiotis, K. Aldape, L. C. Cantley, and Z. Lu. 2012. ERK1/2- dependent phosphorylation and nuclear translocation of PKM2 promotes the Warburg effect. Nature cell biology 14: 1295-1304.

51. Hristova, M., A. Habibovic, C. Veith, Y. M. Janssen-Heininger, A. E. Dixon, M. Geiszt, and A. van der Vliet. 2016. Airway epithelial dual oxidase 1 mediates allergen-induced IL-33 secretion and activation of type 2 immune responses. The Journal of allergy and clinical immunology 137: 1545-1556.e1511.

52. Burgel, P. R., and J. A. Nadel. 2008. Epidermal growth factor receptor-mediated innate immune responses and their roles in airway diseases. The European respiratory journal 32: 1068-1081.

53. Hamilton, L. M., C. Torres-Lozano, S. M. Puddicombe, A. Richter, I. Kimber, R. J. Dearman, B. Vrugt, R. Aalbers, S. T. Holgate, R. Djukanovic, S. J. Wilson, and D. E. Davies. 2003. The role of the epidermal growth factor receptor in sustaining neutrophil inflammation in severe asthma. Clinical and experimental allergy: journal of the British Society for Allergy and Clinical Immunology 33: 233-240.

54. Tan, P. H., P. Sagoo, C. Chan, J. B. Yates, J. Campbell, S. C. Beutelspacher, B. M. Foxwell, G. Lombardi, and A. J. George. 2005. Inhibition of NF-kappa B and oxidative pathways in human dendritic cells by antioxidative vitamins generates regulatory T cells. Journal of immunology (Baltimore, Md. : 1950) 174: 7633-7644. 
55. Boxer, M. B., J. K. Jiang, M. G. Vander Heiden, M. Shen, A. P. Skoumbourdis, N. Southall, H. Veith, W. Leister, C. P. Austin, H. W. Park, J. Inglese, L. C. Cantley, D. S. Auld, and C. J. Thomas. 2010. Evaluation of substituted $N, N^{\prime}$ - diarylsulfonamides as activators of the tumor cell specific $M 2$ isoform of pyruvate kinase. Journal of medicinal chemistry 53: 1048-1055.

56. Qi, W., H. A. Keenan, Q. Li, A. Ishikado, A. Kannt, T. Sadowski, M. A. Yorek, I. H. Wu, S. Lockhart, L. J. Coppey, A. Pfenninger, C. W. Liew, G. Qiang, A. M. Burkart, S. Hastings, D. Pober, C. Cahill, M. A. Niewczas, W. J. Israelsen, L. Tinsley, I. E. Stillman, P. S. Amenta, E. P. Feener, M. G. Vander Heiden, R. C. Stanton, and G. L. King. 2017. Pyruvate kinase M2 activation may protect against the progression of diabetic glomerular pathology and mitochondrial dysfunction. Nat Med 23: 753-762.

Supplemental Table I: primer sequences used in this study

\begin{tabular}{|c|c|c|}
\hline GENES & FORWARD & REVERSE \\
\hline$T s / p$ & TGAGAGCAAGCCAGCTTGTC & GTGCCATTTCCTGAGTACCG \\
\hline Csf2 & ATGCCTGTCACGTTGAATGA & CCGTAGACCCTGCTCGAATA \\
\hline Cxc/1 & TGCGAAAAGAAGTGCAGAGA & TACAAACACAGCCTCCCACA \\
\hline $\mathrm{Cc} / 20$ & AAGACAGATGGCCGATGAAG & AGCCСTTTTCACCCAGTTCT \\
\hline Muc5ac & GCACAGGAGGAAAGAGCATC & AACTTTGCCGAAAACCACAT \\
\hline Col1a1 & CACССTCAAGAGCCTGAGTC & AGACGGCTGAGTAGGGAACA \\
\hline Ppia & AACTTTGCCGAAAACCACAT & GCACAGGAGGAAAGAGCATC \\
\hline
\end{tabular}




\section{CHAPTER 5:}

Glutathionylation chemistry promotes interleukin-1 beta-mediated glycolytic reprogramming and pro-inflammatory signaling in lung epithelial cells

Aboushousha R. ${ }^{*}$, Elko $E^{*}$, Chia SB, Manuel AM, van de Wetering $C$, van der Velden JL, MacPherson MB, Erickson C, Reisz JA, D'Alessandro A, Wouters EFM, Reynaert NL, Lam YW, Anathy V, van der Vliet A, Seward DJ, Janssen-Heininger YMW. * equal contribution

Faseb J. 2021 May 35:e21525. 


\section{ABSTRACT}

Glycolysis is a well-known process by which metabolically active cells, such as tumor or immune cells meet their high metabolic demands. Previously, our laboratory has demonstrated that in airway epithelial cells, the pleiotropic cytokine, interleukin-1 beta (IL1B) induces glycolysis and that this contributes to allergic airway inflammation and remodeling. Activation of glycolysis is known to increase NADPH reducing equivalents generated from the pentose phosphate pathway, linking metabolic reprogramming with redox homeostasis. In addition, numerous glycolytic enzymes are known to be redox regulated. However, whether and how redox chemistry regulates metabolic reprogramming more generally remains unclear. In this study, we employed a multiomics approach in primary mouse airway basal cells to evaluate the role of protein redox biochemistry, specifically protein glutathionylation, in mediating metabolic reprogramming. Our findings demonstrate that IL1B induces glutathionylation of multiple proteins involved in metabolic regulation, notably in the glycolysis pathway. Cells lacking Glutaredoxin-1 (Glrx), the enzyme responsible for reversing glutathionylation, show modulation of multiple metabolic pathways including an enhanced IL1B-induced glycolytic response. This was accompanied by increased secretion of thymic stromal lymphopoietin (TSLP), a cytokine important in asthma pathogenesis. Targeted inhibition of glycolysis prevented TSLP release, confirming the functional relevance of enhanced glycolysis in cells stimulated with IL1B. Collectively, data herein point to an intriguing link between glutathionylation chemistry and glycolytic reprogramming in epithelial cells and suggest that glutathionylation chemistry may represent a therapeutic target in pulmonary pathologies with perturbations in the glycolysis pathway.

\section{Abbreviations}

2-DG: 2-deoxy-D-glucose

ACO2: aconitate hydratase

ALDOA: fructose-bisphosphate aldolase

CCL20: Chemokine (C-C-motif) ligand 20

CS: citrate synthase

ENO1: alpha-enolase

$\mathrm{FH}$ : fumarate hydratase

GAPDH: glyceraldehyde-3- phosphate dehydrogenase

GLRX: glutaredoxin-1

GLRX KO: glutaredoxin knockout cells 
GM-CSF: granulocyte macrophage colony stimulating factor

GPI: glucose-6-phosphate isomerase

GSH: glutathione

HIF: hypoxia-inducible factor

HK1: hexokinase 1

IDH2: isocitrate dehydrogenase

IL-1: interleukin-1

$\mathrm{KC}$ : Chemokine (C-X-C) motif ligand 1

KEGG: kyoto encyclopedia of genes and genomes

LDHA: L-lactate dehydrogenase A

MDH2: malate dehydrogenase, mitochondrial

MMTS: methyl methanethiosulfonate

MTE cells: mouse tracheal epithelial cells

OGDH: 2-oxoglutarate dehydrogenase

PFK: phosphofructokinase

PFKFB3: 6-phosphofructo-2-kinase/fructose-2,6-bisphosphatase 3

PKM: Pyruvate kinase $M$

PGAM1: phosphoglycerate mutase 1

PGK1: phosphoglycerate kinase1

SDH: succinate dehydrogenase

TSLP: thymic stromal lymphopoietin

TPI1: triosephosphate isomerase

WT Ctrl: wild-type control cells 


\section{INTRODUCTION}

Metabolic reprogramming is defined as an altered configuration of cellular metabolism to meet the specialized needs of cells or tissues exposed to distinct stimuli or stresses. Multiple stimuli that reconfigure cellular metabolism and various metabolites that regulate effector functions have been recognized. Aerobic glycolysis, is one example of metabolic reprogramming and is a wellknown feature of metabolically active cells enabling the use of glucose-derived carbons to synthesize macromolecules. Rapidly proliferating tumor cells utilize carbons from glucose to meet their metabolic demands. ${ }^{-}$The activation of immune cells is also accompanied by enhanced glycolysis to permit effector function and cytokine production. For example, the activation of dendritic cells or macrophages with pro-inflammatory stimuli increases glycolysis to produce lipids important for the subsequent activation of $T$ cells,,-4 and enhanced glycolysis contributes to chronic inflammation and autoimmunity. $\frac{5}{}$

Epithelial cells at mucosal barriers play key roles in protecting tissues from environmental stressors and produce a diverse array of antimicrobial factors, mucins, growth factors, chemokines and cytokines to promote homeostasis, tissue repair and afford protection against invading pathogens.,$\underline{7}$ Epithelial cells are therefore metabolically active and are likely to respond to diverse stimuli through metabolic reprogramming. Previous work from our laboratory and others has shown that aerobic glycolysis is a key feature of epithelial cell activation or regeneration, akin to observations in immune cells. $\underline{8}, \underline{9}$ Notably, we demonstrated that interleukin-1 (IL1) is a prominent inducer of glycolysis in airway epithelial cells and in settings of allergic inflammation. $\underline{8}$ Enhanced glycolysis contributed to allergic inflammation, remodeling, and hyperresponsiveness in a mouse model of house dust mite-induced allergic airway disease. Furthermore, increases in lactate, a marker of glycolysis, were observed in sputum samples from asthmatics and directly correlated with increases in airway neutrophils and disease severity. $\underline{8}$

Glycolytic reprogramming is closely linked to changes in redox homeostasis. Enhanced glycolysis offers protection against oxidative stress owing to increases in NADPH derived from the pentose phosphate pathway, an offshoot of glycolysis. Notably, tumor cells augment glycolysis to combat enhanced oxidative stress and to maintain homeostasis of glutathione, $\underline{10}$ a major cellular redox system. .11 Numerous proteins in the glycolysis cascade are redox regulated, suggesting that oxidative events contribute to increases in glycolysis. Of note, oxidation of the glycolysis enzymes glyceraldehyde-3-phosphate dehydrogenase,,$\underline{12}$, $\underline{13}$ 6-phosphofructo-2-kinase/fructose-2,6bisphosphatase 3 (PFKFB3), $\underline{14}$ triosephosphate isomerase, $\underline{15}$ lactate dehydrogenase, and enolase $^{16}$ have been demonstrated, in association with changes in their function. 
One facet of glutathione biochemistry that remains less well recognized is its ability to regulate protein oxidation. Under conditions of oxidative stress, reactive cysteines are oxidized to sulfenic acid and other intermediates. 17 Glutathione can react with sulfenic acid intermediates leading to glutathionylated moieties. Glutathione disulfide, the oxidized form of glutathione, also can induce protein glutathionylation (also known as S-glutathionylation or protein mixed disulfides). Glutathionylation is critical in the protection from irreversible overoxidation of protein cysteines, and also affects protein structure and function. Glutaredoxin-1 is a cellular enzyme that deglutathionylates its target proteins and reestablishes the reduced protein thiol groups. $\underline{18}, \underline{19}$ To date, it remains unclear whether glutathionylation affects glycolytic reprogramming and subsequent pro-inflammatory responses. Furthermore, the extent to which changes in glycolysis and other metabolic pathways occur in primary epithelial cells in response to an innate immune stimulus remains incompletely known. In the present study, we sought to investigate the importance of glutathionylation chemistry in metabolic reprogramming of primary airway basal cells via the comparative evaluation of WT or Glrx ${ }^{-/}$cells. Given the importance of IL1B as an innate immune activator, its ability to induce glycolysis in epithelial cells and prior observations that IL1B induces oxidative stress, $\underline{20}, \underline{21}$ we utilized IL1B as the stimulus to assess metabolic reprogramming using multiple-omics approaches, while emphasizing glycolysis. Our results demonstrate that IL1B-stimulated glycolysis is enhanced in the absence of Glrx and that multiple proteins in the glycolysis pathway are targets of glutathionylation.

\section{Methods}

\section{Reagents and antibodies}

Recombinant mouse IL1B was purchased from R\&D systems, Minneapolis, MN, USA. Anti-GSH antibody was acquired from Virogen, Watertown, MA, USA. GAPDH and HK1 antibody were from Cell signaling technology, Danvers, MA, USA. [U- $\left.{ }^{13} \mathrm{C}\right]$ labeled glucose and 2-deoxy-D-glucose (2DG) were obtained from Sigma-Aldrich, St. Louis, MO, USA. TSLP, GM-CSF, CCL20/MIP-3 alpha, and CXCL1/KC mouse ELISA kits were purchased from R\&D systems, Minneapolis, MN, USA.

\section{Mouse airway basal cell cultures and exposure to IL1B}

Primary mouse tracheal epithelial (MTE) cells were isolated from wild-type (WT) C57BL6/NJ mice or C57BL6/NJ mice lacking Glrx gene (GLRX KO) and cultured as previously described. $\underline{2}$, 23 Cells were grown on transwell inserts with media change every 2 days until forming a monolayer depicted by stable transepithelial electrical resistance (TEER) measurement. Media was then 
switched to plain DMEM:F12 media overnight followed by the incubation with IL1B (10 ng/mL) or $0.1 \%$ bovine serum albumin (BSA) in phosphate-buffered saline (PBS) (vehicle control) for 24 hours. To test the effect of glycolysis inhibition on pro-inflammatory cytokines release, cells were incubated in media containing $10 \mathrm{mM}$ 2-DG for 1 hour before stimulation with IL1B.

\section{${ }^{13} \mathrm{C}$-glucose labeling and metabolomics analysis}

MTE cells were grown to confluency as described above. The cells were washed and incubated overnight in plain DMEM:F12 media containing $6 \mathrm{mM}$ glucose and $2 \mathrm{mM}$ glutamine. The next day, cells were washed three times in media with no glucose, then, treated with IL1B or $0.1 \%$ BSA in PBS in media containing $6 \mathrm{mM}$ of ${ }^{13} \mathrm{C}$-glucose and $2 \mathrm{mM}$ glutamine. After 24 hours, cells were washed with PBS and pelleted. The supernatant was removed, and pellets were snap frozen in liquid nitrogen. Mass spectrometry-based metabolomics was performed at the University of Colorado, School of Medicine Metabolomics Core. Metabolites were extracted from frozen cell pellets by vortexing 30 minutes in the presence of ice-cold 5:3:2 methanol:acetonitrile:water $(\mathrm{v} / \mathrm{v} / \mathrm{v})$ at 2 million cells per $\mathrm{mL}$ as described. $\stackrel{24}{ }$ Supernatants were clarified via centrifugation at $18000 \mathrm{~g}$ for 10 minutes at $4^{\circ} \mathrm{C}$, then, analyzed on a Thermo Vanquish UHPLC coupled to a Thermo $Q$ Exactive mass spectrometer using a 5 minutes $\mathrm{C} 18$ gradient in positive and negative ion modes (separate runs) exactly as previously described. $\underline{25}$, $\underline{2}$ Quality control, metabolite annotation, and peak area integration were performed using Maven (Princeton University) as described. $\underline{26}, \underline{27}$ Isotopologue distribution was plotted in GraphPad Prism 8.0. Heat maps were created by using a range scale function to scale the data set and visualized using the ComplexHeatmap package in R. $\underline{28}, \underline{29}$

\section{DNA array}

Microarray samples were prepared with a $50 \mathrm{ng}$ RNA input as previously described using the Ovation Pico WTA System V2 (PN3302-12). Samples were biotinylated (a $5.5 \mu \mathrm{g}$ input) with the Encore Biotin Module Part No. 4200-12. Briefly, Encore Biotin Module employs a proprietary fragmentation and labeling process that combines enzymatic and chemical processes for the preparation of labeled cDNA suitable for hybridization to Affymetrix GeneChip. Efficiency of the fragmentation and labeling reactions were verified using NeutrAvidin (10 mg/mL) with a gel-shift assay. An input of $2.5 \mu \mathrm{g}$ of Biotin labeled single primer isothermal amplification (SPIA) cDNA was combined with a hybridization mix, injected into Mouse Clariom $S$ arrays, and placed in the Affymetrix GeneChip Hybridization Oven 645 at $45^{\circ} \mathrm{C}$ and 60 RPM for 16.5 hours overnight. Arrays were stained using the Affymetrix GeneChip Fluidics Station 450 and scanned with the 7G 
Affymetrix GeneChip Scanner 3000. DNA array was normalized using the Transcriptome Analysis Console software (Thermo Fisher). The data were loaded into $\mathrm{R}^{\underline{28}}$ using the Biobase package $\underline{30}$ and annotated using the $\mathrm{R}$ package affycoretools. 1 Differential expression between groups was calculated using the LIMMA package in $\mathrm{R}^{32}$ and a $P$ value of less than .05 and fold change greater than 2 were used as a cutoffs. Heat maps of mRNA expression were created using the ComplexHeatmap package in $\mathrm{R} . \underline{29}$

\section{Identification of glutathionylated proteins using GLRX-catalyzed cysteine derivatization and mass spectrometry}

Cells were lysed in $50 \mathrm{mM}$ Tris buffer pH 7.4 with $150 \mathrm{mM} \mathrm{NaCl}, 1 \%$ Igepal-630, $5 \mathrm{mM}$ ethylenediaminetetraacetic acid (EDTA), $0.1 \%$ sodium dodecyl sulfate (SDS), $0.5 \%$ sodium deoxycholate, and $80 \mathrm{mM}$ methyl methanethiosulfonate (MMTS). A $2 \mathrm{~mL}$ of blocking buffer containing $100 \mathrm{mM}$ HEPES, pH 7.5, $1 \mathrm{mM}$ EDTA, 2.5\% SDS, and $80 \mathrm{mM}$ MMTS was added to each sample and they were incubated at $50^{\circ} \mathrm{C}$ for 20 minutes to block free thiol groups on cysteine residues. Samples containing $1 \mathrm{mg}$ protein were acetone-precipitated three times with $70 \%$ icecold acetone to remove excess MMTS from the solution and resuspended in binding buffer (10 mM HEPES pH 8.0, 0.1 mM EDTA, and 0.1\% SDS). Each sample was incubated with $1 \mathrm{mM}$ $\mathrm{NADPH}, 35 \mu \mathrm{g} / \mathrm{mL}$ glutathione reductase (Sigma-Aldrich, St. Louis, MO), $25 \mu \mathrm{g} / \mathrm{mL}$ wild-type mouse $\mathrm{GLRX}, \underline{33}$ and $0.2 \mathrm{mM}$ reduced $\mathrm{GSH}$ for 30 minutes at $37^{\circ} \mathrm{C}$ to remove the $\mathrm{GSH}$ adduct from cysteine thiol groups. Negative control samples were incubated in the absence of GLRX and GSH. Proteins containing free thiol groups following GLRX-derivatization were pulled down by rotating each sample with $10 \mathrm{mg}$ of Thiopropyl Sepharose beads (Sigma-Aldrich, St. Louis, MO) for 1 hour at room temperature. The beads were washed three times with $10 \mathrm{mM} \mathrm{HEPES}(\mathrm{pH}$ 8.0), $0.1 \mathrm{mM}$ EDTA, $1 \% \mathrm{SDS}$, and $250 \mathrm{mM} \mathrm{NaCl}$ and proteins were eluted from the beads by boiling the samples in $1 \times$ Laemmli buffer for 8 minutes at $95^{\circ} \mathrm{C}$ before loading the samples onto $10 \%$ of acrylamide gels. Gels were stained with Coomassie blue, bands were excised from the gel, and washed with $50 \%$ of methanol and $5 \%$ of acetic acid overnight. The proteins were reduced with $10 \mathrm{mM}$ dithiothreitol and alkylated with $100 \mathrm{mM}$ iodoacetamide followed by digestion with Promega sequencing grade modified trypsin $(20 \mathrm{ng} / \mu \mathrm{L})$ (Promega, Madison, WI). The digested samples were analyzed on the Q Exactive Plus mass spectrometer coupled to an EASYnLC 1200 (Thermo Fisher Scientific). Samples were loaded onto a $100 \mu \mathrm{m}$ x $125 \mathrm{~mm}$ capillary column packed Halo C18 (2.7 $\mu \mathrm{m}$ particle size, $90 \mathrm{~nm}$ pore size, Michrom Bioresources, CA, USA) at a flow rate of $300 \mathrm{~nL} / \mathrm{min}$. The column end was laser pulled to a $\sim 3 \mu \mathrm{m}$ orifice and packed with minimal amount of $5 \mu \mathrm{m}$ Magic $\mathrm{C} 18 \mathrm{AQ}$ before packing with the $2.7 \mu \mathrm{m}$ particle size 
chromatographic materials. Peptides were separated by a gradient of $0 \%-35 \% \mathrm{CH} 3 \mathrm{CN} / 0.1 \%$ FA over 60 minutes, 35\%-80\% CH3CN/0.1\% FA in 1 minute, and then $80 \% \mathrm{CH} 3 \mathrm{CN} / 0.1 \% \mathrm{FA}$ for 4 minutes, followed by an immediate return to $0 \% \mathrm{CH} 3 \mathrm{CN} / 0.1 \% \mathrm{FA}$ and a hold at $0 \%$ $\mathrm{CH} 3 \mathrm{CN} / 0.1 \%$ FA. Samples were randomized in run order. Mass spectrometry data were acquired in a data-dependent "Top 10" acquisition mode with lock mass function activated $(\mathrm{m} / \mathrm{z}$ 371.1012; use lock masses: best; lock mass injection: full MS), in which a survey scan from $\mathrm{m} / z$ 350-1600 at 70000 resolution (AGC target 1e6; max IT 100 ms; profile mode) was followed by 10 higherenergy collisional dissociation (HCD) tandem mass spectrometry (MS/MS) scans on the most abundant ions at 35000 resolution (loop count = 10; AGC target 5e4; max IT 100 ms; centroid mode). MS/MS scans were acquired with an isolation width of $1.2 \mathrm{~m} / \mathrm{z}$ and a normalized collisional energy of 35\%. Dynamic exclusion was enabled (peptide match: preferred; exclude isotopes: on; underfill ratio: $1 \%$ ). Minimum AGC target $=1 \mathrm{e}$. Product ion spectra were searched using SEQUEST in the Proteome Discoverer 2.2 against a Uniprot Mus musculus protein database downloaded on June, 2017) with the "Basic" Processing and Consensus workflows. Appropriate fix and variable modifications were considered. Target Decoy PSM Validator was included in the workflow to limit the false discovery rate to less than $1 \%$.

\section{Western blot analysis}

Cell lysates were prepared and protein concentration was determined using the Bio-Rad DC protein estimation kit. Equal amounts of proteins were resolved using sodium dodecyl sulfate (SDS)-polyacrylamide gel electrophoresis and transferred to polyvinylidene difluoride (PVDF) membranes. Overnight incubation with GSH, GAPDH or HKI antibody was performed before membranes were developed using peroxidase-conjugated secondary antibodies and imaged with chemiluminescence. For immunoprecipitating glutathionylated proteins, $200 \mu \mathrm{g}$ of proteins were used in the pull down with one sample incubated with $50 \mathrm{mM}$ DTT as a negative control.

\section{Statistical analysis}

Data in this manuscript are expressed as means \pm SEM. Significant differences between groups were determined using the GraphPad Prism (GraphPad 8.2.1) software and analyzed by one-way ANOVA with a Tukey's post hoc correction test for multiple comparisons. $P$ values lower than .05 were considered significant. 


\section{RESULTS}

\section{Increased glutathionylation of multiple classes of proteins in response to interleukin-1 beta (IL1B) and exacerbation in the absence of glutaredoxin-1 (GIrx)}

We previously demonstrated that IL1B is a key inducer of glycolysis in lung epithelial cells which enhances production of pro-inflammatory cytokines and augments responsiveness to house dust mite allergen. $\underline{8}, \underline{34}$ Previous studies have also demonstrated that IL1B induces oxidative stress. $\underline{20}$, 21 We therefore explored whether increases in glutathionylation occur in response to stimulation with IL1B. In epithelial cells exposed to IL1B for 24 hours, overall glutathionylation was increased (Figure 1A). Comparative evaluation of epithelial cells lacking Glrx ${ }^{-/}$demonstrated further increases in glutathionylation compared to control cells (Figure $\underline{1 \mathrm{~A}}$ ), consistent with the physiological role of GLRX as a deglutathionylase. We next identified the proteins that were glutathionylated in this experimental setting, by blocking reduced thiols with MMTS followed by GLRX-mediated cysteine derivatization, thiopropyl sepharose bead capture and identification of captured protein by mass spectrometry. Although the absolute number of glutathionylated proteins differed between two independently conducted experiments, a substantial portion of captured proteins overlapped, including 102 proteins in WT control cells, and 277 proteins in WT cells stimulated with IL1B. In control Glrx ${ }^{-/}$cells, 167 glutathionylated proteins were detected, while in Glrx ${ }^{-/}$cells stimulated with IL1B this number increased to 179 proteins (Figure $\underline{1 \mathrm{~B}}$, Supplementary Figure $\underline{1 \mathrm{~A}})$. Four glutathionylated proteins were uniquely detected in WT control cells, and 83 glutathionylated proteins were unique to WT cells stimulated with IL1B, whereas 9 proteins were uniquely detected in control Glrx $x^{-/}$cells and 19 in Glrx ${ }^{-/}$cells treated with IL1B (Supplementary Figure 1A). Pathway enrichment analysis performed using the Kyoto Encyclopedia of Genes and Genomes (KEGG) database ${ }^{35}$ revealed that the identified glutathionylated proteins in each group occurred in overlapping categories (Figure $\underline{1 \mathrm{C}}$ ) with hits predominantly occurring in metabolic pathways, including glycolysis (Supplementary Figure $\underline{1 \mathrm{~B}}$ ). As expected, GLRX itself also was detected in all four groups (Supplementary Figure $\underline{1 \mathrm{~A}}$ ), likely reflecting the capture of exogenous GLRX used in the derivatization reaction. Immunoprecipitation of glutathionylated proteins using an anti-GSH antibody followed by Western blotting validated some of these glutathionylation targets in the glycolysis pathway (Figure 1D). Overall, these data show that IL1B increases total protein glutathionylation in airway epithelial cells that is exacerbated in the absence of GLRX and suggest that protein glutathionylation affects multiple biochemical pathways, with a notable enrichment of proteins that govern cellular glucose metabolism. 


\section{Targeted metabolomics identification of metabolites in epithelial cells stimulated with IL1B}

Given that glutathionylated proteins were enriched in metabolic pathways, we next conducted targeted metabolomics analysis in order to unravel whether the abundance of metabolites changed in epithelial cells stimulated with IL1B, and whether these patterns were affected in the absence of GLRX. Heat maps demonstrate a statistically significant difference in the abundance of multiple metabolites in MTE cells exposed to IL1B, with a further enrichment of some metabolites occurring at baseline or in response to IL1B in Glrx ${ }^{-/}$cells compared to WT counterparts (Figure 2). KEGG pathway analyses revealed significant differences in metabolites between WT control and IL1B-exposed cells in the categories of aminoacyl t-RNA biosynthesis, alanine, aspartate and glutamate metabolism, arginine biosynthesis, phenylalanine, tyrosine and tryptophan biosynthesis, glutathione metabolism and valine, leucine, and isoleucine biosynthesis (Supplementary Figure 2). Compared to WT cells exposed to IL1B, IL1B-exposed Glrx-deficient cells revealed increases in metabolites representing some of these pathways along with additional metabolites occurring in the TCA cycle as well as arginine and proline metabolism (Supplementary Figure 2 ). 


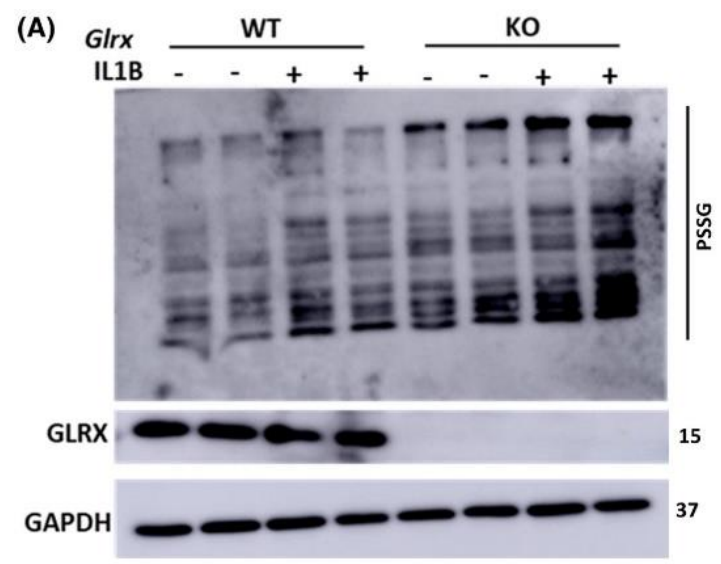

(C)

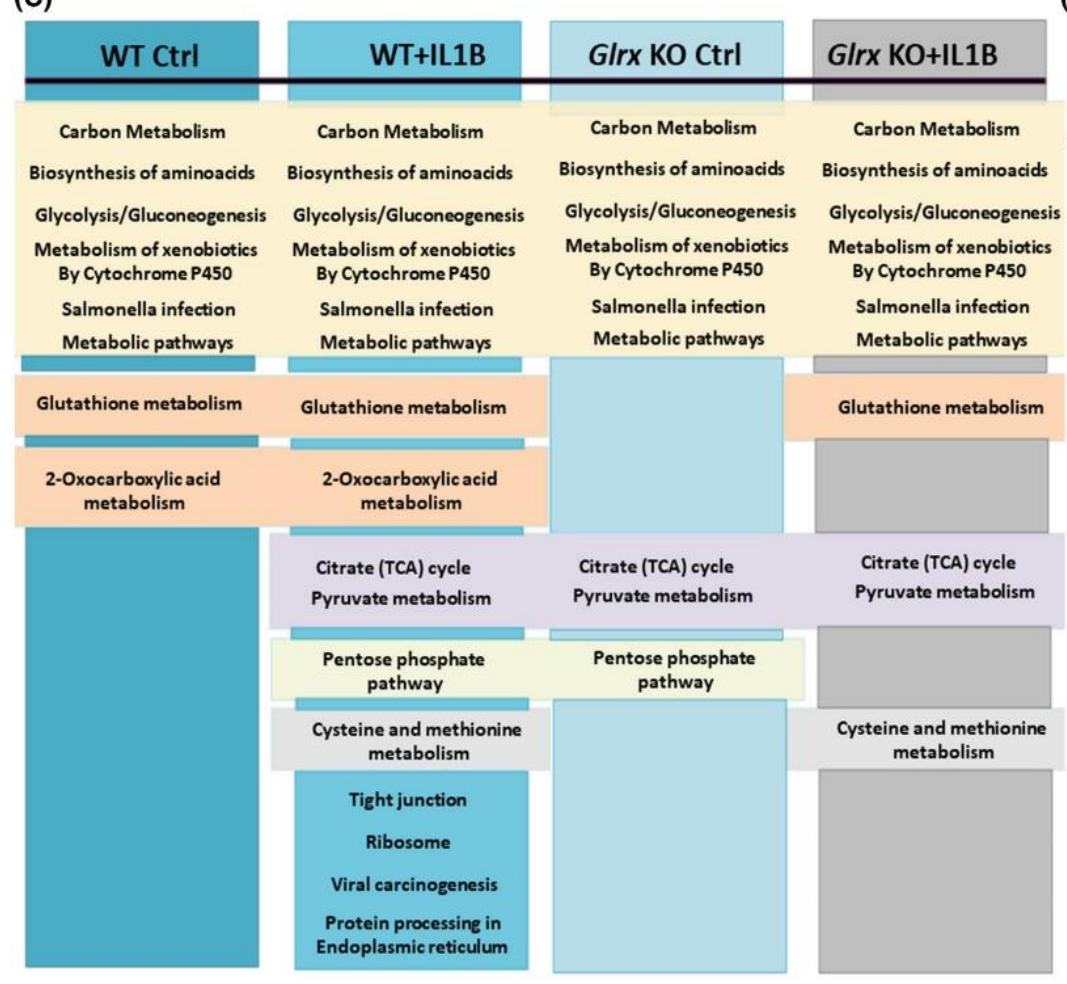

(B)

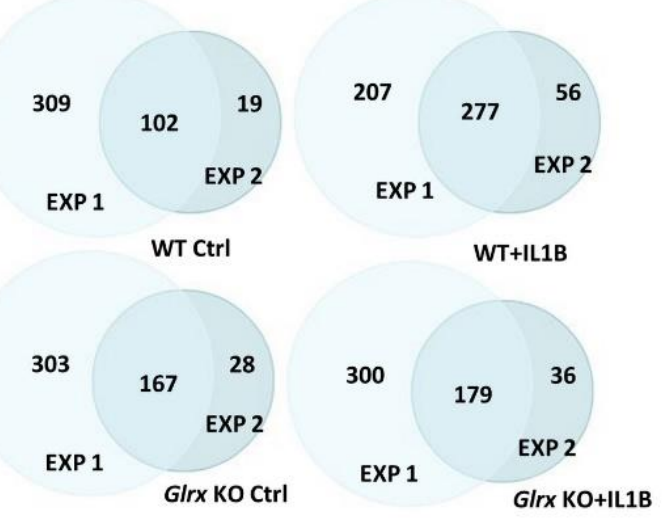

(D)

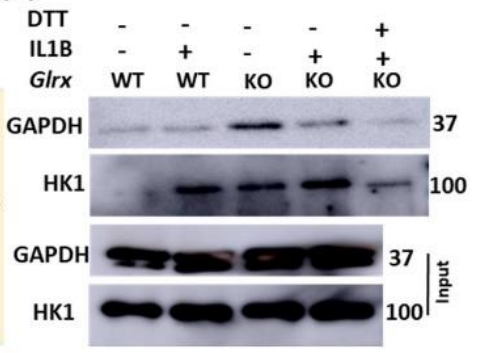

FIGURE 1: Assessment of IL1B-mediated effects on protein glutathionylation in mouse airway basal cells and the impact of ablation of Glrx. A, Western blot of total glutathionylated proteins in WT and Glrx ${ }^{-/}$airway basal cells treated with $10 \mathrm{ng} / \mathrm{mL}$ IL1B for 24 hours or vehicle control. Top: Anti-GSH, Middle: GLRX, bottom: GAPDH as a loading control. B, Venn diagrams showing numbers of glutathionylated proteins in WT ctrl, WT + IL1B, Glrx ${ }^{-/}$Ctrl, and Glrx ${ }^{-/}+$IL1B groups appearing in two independent experiments. Results in $\mathrm{C}$ and supplementary Figure 1 reflect analyses done on only the overlapping proteins between the two experiments. C, KEGG pathways analysis for glutathionylated proteins appearing in each group. D, Confirmation of select glutathionylation target using immunoprecipitation with an GSH antibody followed by Western blotting for GAPDH and HK1. Dithiothreitol (DTT) was added to the cell lysate prior to immunoprecipitation as a negative control. Bottom input panels: Western blots of whole cell lysates. 


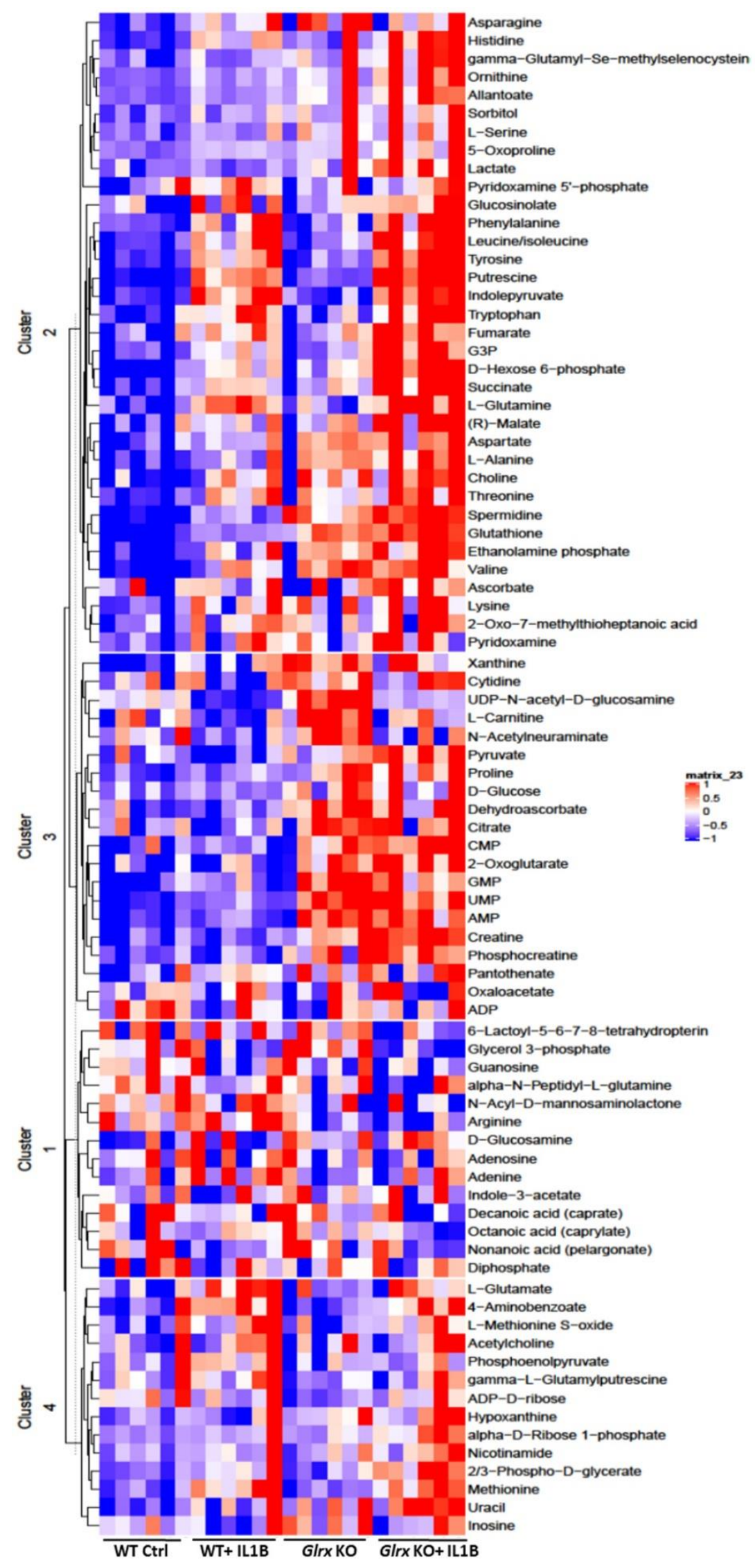

FIGURE 2: Metabolomics analysis in WT or Glrx ${ }^{-}$airway basal cells stimulated with IL1B. Heat map showing the top differentially regulated metabolites appearing in each of the four groups. Each column represents a biological replicate. The heat map was generated using range scaled metabolomics data with a $P$ value $<.05$ for one of the four comparisons 


\section{Assessment of differential gene expression in epithelial cells stimulated with IL1B}

Based on the complex differences in glutathionylated proteins and metabolites observed in epithelial cells exposed to IL1B, we next sought to explore whether gene expression changes induced by IL1B mirrored some of these changes. We therefore conducted a DNA array study to address this question. As anticipated, the most significantly upregulated mRNAs reflected genes important in inflammation and innate host responses, including the TNF signaling pathway, (Figure $\underline{3}$, Supplementary Figure $\underline{3}$ ). KEGG pathway analysis revealed significant differences between control and IL1B-exposed cells in multiple categories, and expression changes in genes within many of these classes were enhanced in Glrx $x^{-\alpha}$ cells (Supplementary Figure $\underline{3}$ ). Of note, expression of genes in glycolysis/gluconeogenesis and metabolic pathways were preferentially enhanced in Glr $x^{-\alpha}$ cells compared to the respective control groups (Supplementary Figures $\underline{3}$ and 4), consistent with targets observed in these pathways identified in the glutathionylated proteome and via targeted metabolomics.

\section{Multi-omics pathway analysis in IL1B-stimulated epithelial cells}

The assessment of the glutathionylated proteome, metabolome, and gene expression profiles revealed significant overlapping KEGG pathway enrichments across all three-omics platforms. Notably, the glycolysis pathway contained numerous hits across all three platforms. Intracellular levels of hexose-6-phosphate were significantly increased in Glrx ${ }^{-/}$cells compared to WT controls. In addition, hexose-6-phosphate, glyceraldehyde-3-phosphate, pyruvate, and lactate were significantly elevated in Glrx ${ }^{-/}$cells after IL1B stimulation compared to WT counterparts (Figure $\underline{4 A}$, right). Glrx ${ }^{-\alpha}$ cells showed constitutive increases in a number of glycolysis genes relative to WT cells including Hk1 (hexokinase1), Pfk (phosphofructokinase), Aldoa (fructosebisphosphate aldolase), Tpi1 (triosephosphate isomerase), Gapdh (glyceraldehyde-3-phosphase dehydrogenase), Pgk1 (phosphoglycerate kinase1), Eno1 (enolase), Pkm (pyruvate kinase M), and Lhda (lactate dehydrogenase A). Expression of some of these genes also increased in response to IL1B both in WT and Glr $x^{-/}$cells, consistent with earlier findings. ${ }^{-}$Interestingly, numerous of the enzymes in the glycolysis cascade were also identified as targets for glutathionylation, without any clear differences between genotypes or treatment groups (Figure 4A, left, green boxes). In addition to the modulation of the glycolysis pathway, a number of TCA metabolites including succinate, fumarate, and malate were more robustly increased in IL1B-exposed Glrx $x^{-/}$cells compared to the WT groups (Figure $\underline{4 \mathrm{~B}}$, bottom). These alterations were accompanied by changes in mRNA expression in TCA cycle genes (Figure $\underline{4 B}$, top). Interestingly, citrate synthase, aconitase, isocitrate dehydrogenase, oxoglutarate dehydrogenase, 
succinate dehydrogenases, and malate dehydrogenase were all identified as targets for glutathionylation in one or more of the treatment groups or genotypes compared (Figure $\underline{4 \mathrm{~B}}$, top, green boxes). Together, these multi-omics pathway analyses point to a prominent regulatory role for glutathionylation in the reconfiguration of the glycolysis pathway and the TCA cycle.

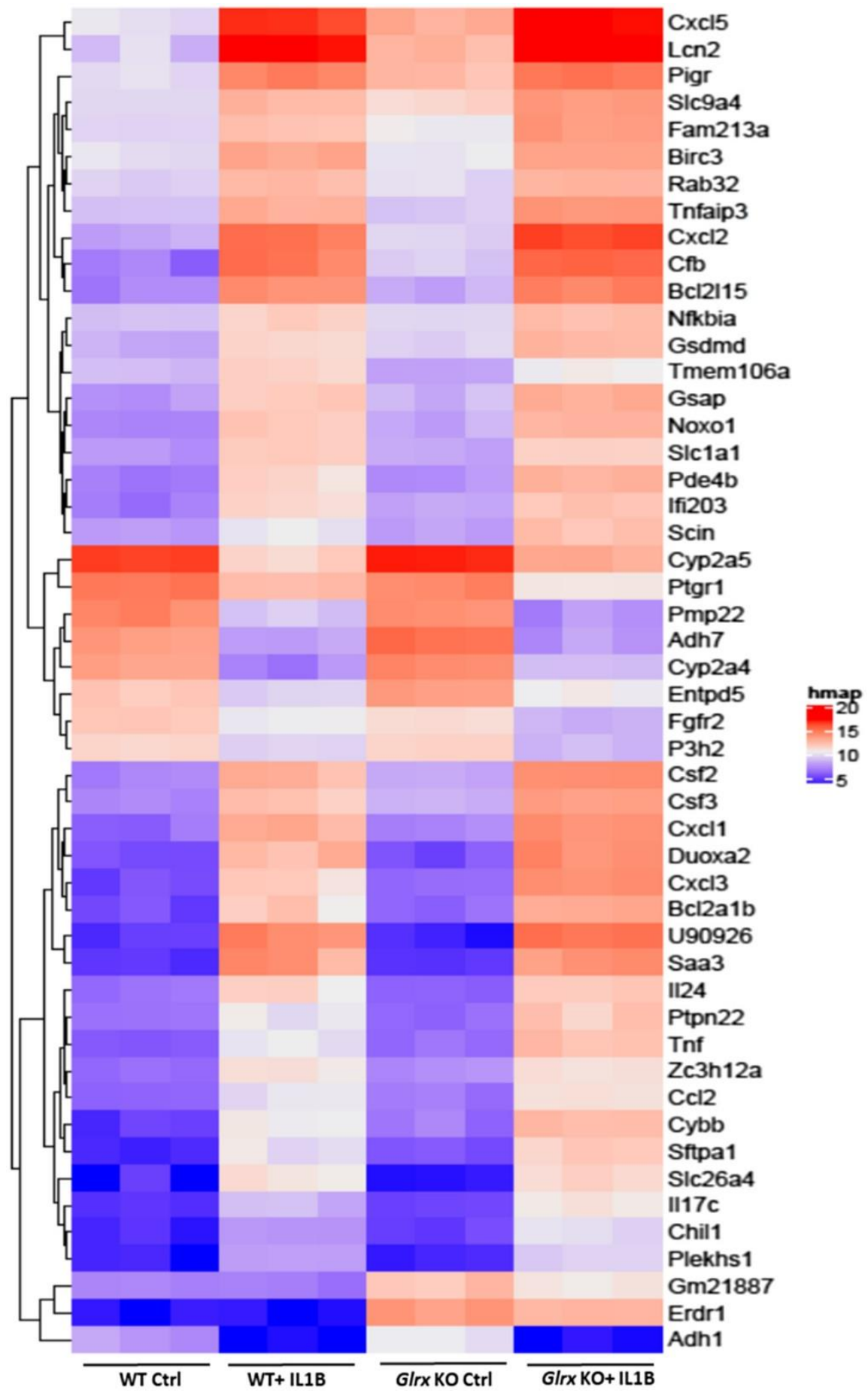

FIGURE 3: Microarray analysis in WT or Glrx ${ }^{-1}$ airway basal cells stimulated with IL1B. Heat map for the top 50 differentially regulated genes in the four groups. $P$ values were calculated by comparing all four comparisons using Linear Models for Microarray Data (LIMMA) 
(A)
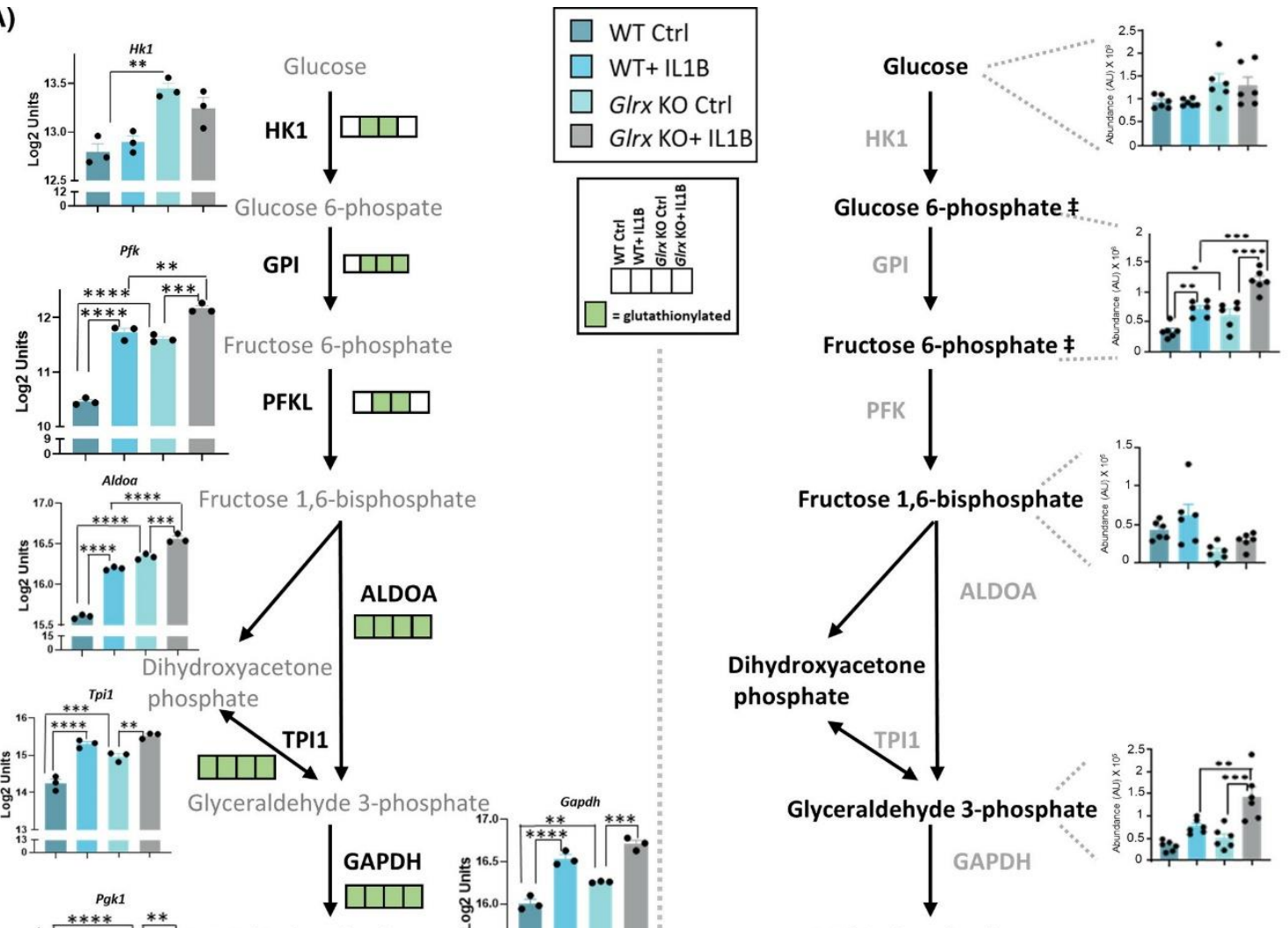

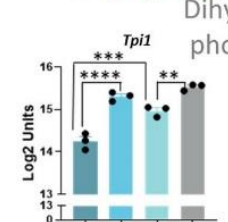

ihydroxyacetone पाD

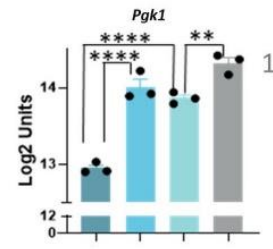

1,2-bisphosphoglycerate

Glyceraldehyde
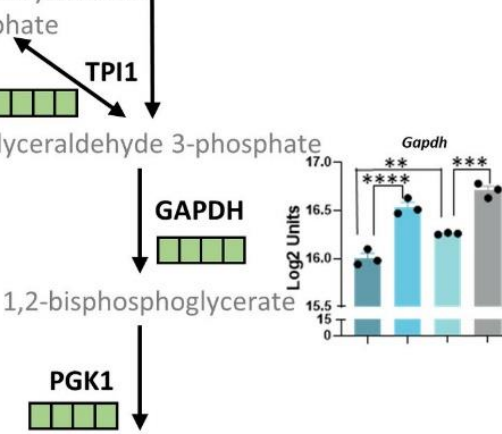

3-phosphoglycerate
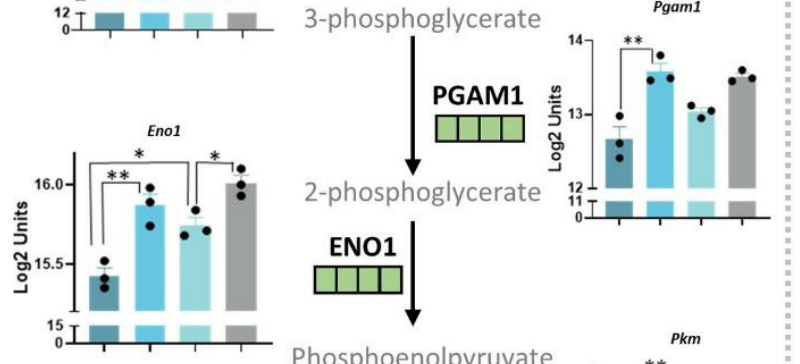

Phosphoenolpyruvate
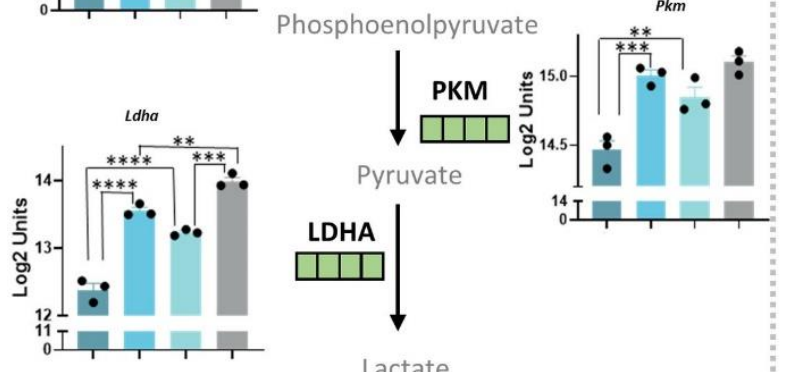

\section{1,2-bisphosphoglycerate}
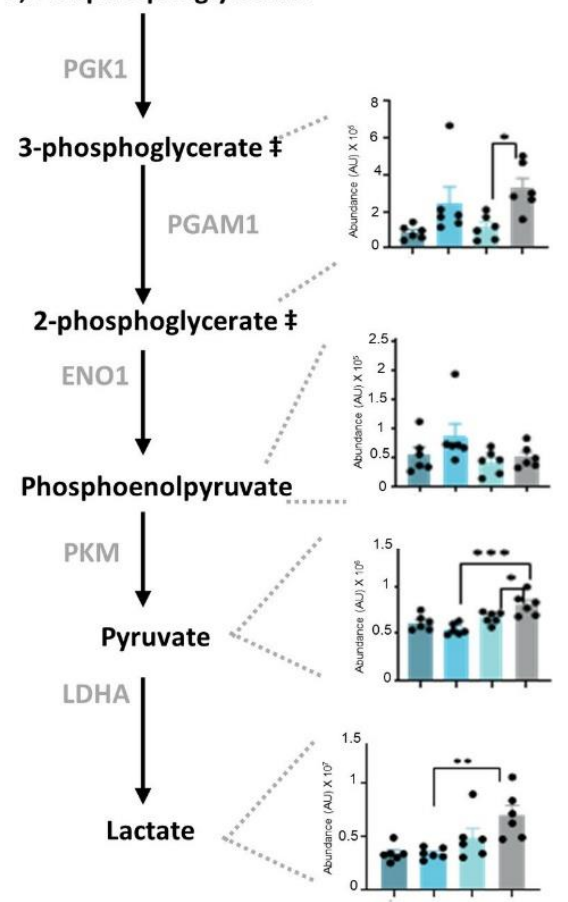
(B)
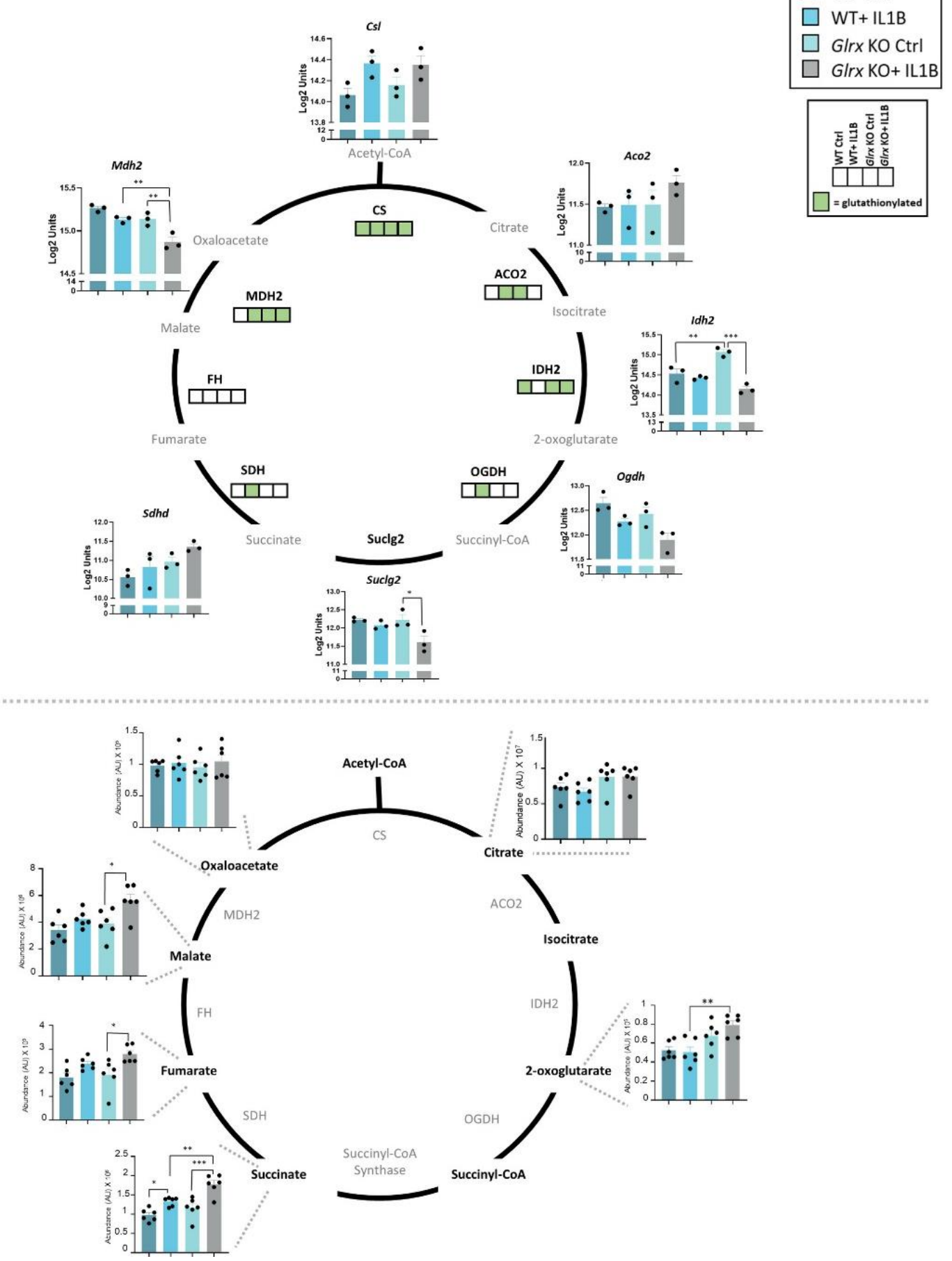

FIGURE 4A: Multi-omics integration of KEGG pathways affected in airway basal cells stimulated with IL1B that are affected by GLRX status. Visualization of hits in the TCA cycle and glycolysis pathway identified in the -omics platforms described in Figures 1-3-1-3. A, Glycolysis pathway; right panel shows the total metabolites and left panel shows the microarray gene expression data as well as glutathionylated protein hits in each of the four groups (present; green, absent; white). \#\# Metabolites measured are hexose-6 
phosphate (top) and phosphoglycerate (bottom). Hk1: hexokinase1, Pfk: phosphofructokinase, Aldoa: fructose-bisphosphate aldolase A, Tpi1: triosephosphate isomerase, Pgk1: phosphoglycerate kinase1, Gapdh: glyceraldehyde-3-phosphate dehydrogenase, Pgam1: phosphoglycerate mutase1, Eno1: enolase1, Pkm: pyruvate kinase M, Ldha: lactate dehydrogenase A, HK1: hexokinase1, GPI: glucose-6phosphate isomerase, PFK: phosphofructokinase, ALDOA: Fructose-bisphosphate aldolase, TPI1: triosephosphate isomerase, GAPDH: glyceraldehyde-3-phosphate dehydrogenase, PGK1: phosphoglycerate kinase1, PGAM1: phosphoglycerate mutase 1, ENO1: alpha-enolase, PKM: pyruvate kinase M, LDHA: L-lactate dehydrogenase A 4B: B, TCA cycle; bottom panel shows the total metabolites and top panel shows the microarray gene expression data as well as glutathionylated protein hits in each of the four groups (present; green, absent; white). Csl: citrate synthase, Aco2: aconitate hydratase, mitochondrial, Idh2: isocitrate dehydrogenase, mitochondrial, Ogdh: 2-oxoglutarate dehydrogenase, mitochondrial, Suclg2: succinate coA ligase, subunit G, Sdhd: succinate dehydrogenase, mitochondrial, Mdh2: malate dehydrogenase, mitochondrial. CS: citrate synthase, ACO2: aconitate hydratase, IDH2: isocitrate dehydrogenase, $\mathrm{OGDH}$ : 2-oxoglutarate dehydrogenase, $\mathrm{SDH}$ : succinate dehydrogenase, $\mathrm{FH}$ : fumarate hydratase, MDH2: malate dehydrogenase, mitochondrial. ${ }^{*} P<.05$, ${ }^{* *} P<.01,{ }^{* * *} P<.001$, ${ }^{* * * *}$ $P<.0001$ (ANOVA).

\section{Ablation of Glrx enhances glycolysis in airway epithelial cells and regulates pro- inflammatory signaling induced by IL1B}

In order to corroborate that glycolysis was increased in the absence of Glrx, we traced carbons from $\left[\mathrm{U}-{ }^{13} \mathrm{C}\right]$ glucose through glycolysis and TCA cycle. Consistent with unlabeled metabolite analyses, ${ }^{13} \mathrm{C}$-hexose phosphate and ${ }^{13} \mathrm{C}$-lactate were increased 24 hours post IL1B, with further increases in ${ }^{13} \mathrm{C}$-hexosephosphate, ${ }^{13} \mathrm{C}$-glyceraldehyde-3-phosphate, ${ }^{13} \mathrm{C}$ pyruvate, and ${ }^{13} \mathrm{C}^{-}$ lactate occurring in Glrx ${ }^{-\alpha}$ cells stimulated with IL1B (Figure $\underline{5 \mathrm{~A}}$ ), along with more pronounced increases in lactate detected in the media from $\mathrm{Glrx}^{-/}$cells detected in parallel experiments not using ${ }^{13} \mathrm{C}$-glucose (Figure $\underline{5 \mathrm{~B}}$ ). In addition, ${ }^{13} \mathrm{C}$-fumarate, ${ }^{13} \mathrm{C}$ malate, and ${ }^{13} \mathrm{C}$-succinate were increased in response to IL1B stimulation in WT and/or Glrx ${ }^{-/}$cells (Figure $\underline{5 \mathrm{C}}$ ). A time course analysis revealed time-dependent changes in labeled metabolites between the groups (Supplementary Figure $\underline{5}$ ). Collectively these findings demonstrate that carbons derived from glucose enter the glycolysis pathway and are used to fuel the TCA cycle, in response to IL1B and that glucose usage in these pathways is enhanced in the absence of Glrx.

We previously demonstrated that IL1B-induced glycolysis contributes to enhanced expression of select pro-inflammatory genes in epithelial cells notably of thymic stromal lymphopoietin (TSLP) and granulocyte monocyte colony stimulating factor (GM-CSF). Based on the findings that 
glycolysis is enhanced in the absence of Glrx we next addressed whether gene expression and secretion of these pro-inflammatory mediators were enhanced in the absence of Glrx. Results in Figure $\underline{6 \mathrm{~A}}$, show that IL1B induced increased gene expression of Ts/p, Csf2 (encoding GM-CSF), Ccl20, and Cxcl1 (encoding KC), with exacerbated increases of $T s / p, C s f 2$, and $C x C / 1$ apparent in IL1B-stimulated cells lacking Glrx. In addition, IL1B-mediated secretion of TSLP was higher in Glr $x^{-\alpha}$ cells compared to WT counterparts along with higher secretion of GM-CSF. In contrast, IL1B-induced CCL20 and KC were similar between WT and Glr $x^{-/}$cells (Figure $\underline{6 B}$ ). Administration of 2-deoxyglucose (2-DG), an inhibitor of glycolysis, potently inhibited secretion of TSLP and GM-CSF irrespective of genotype, and also attenuated the release of CCL20 and KC in media (Figure $\underline{6 B}$ ). These findings demonstrate that enhanced glycolysis induced by IL1B in epithelial cells lacking Glrx results in exaggerated expression of TSLP, a mediator of severe inflammation in asthma. $.36,37$ 
(A)
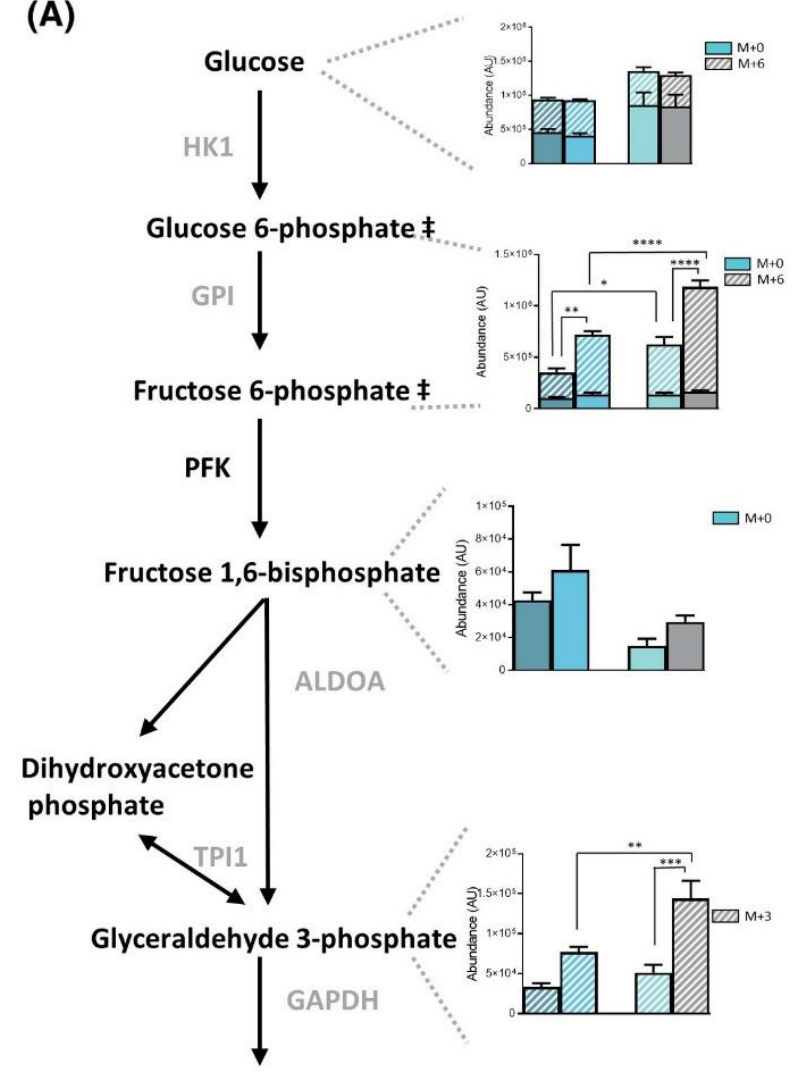

1,2-bisphosphoglycerate

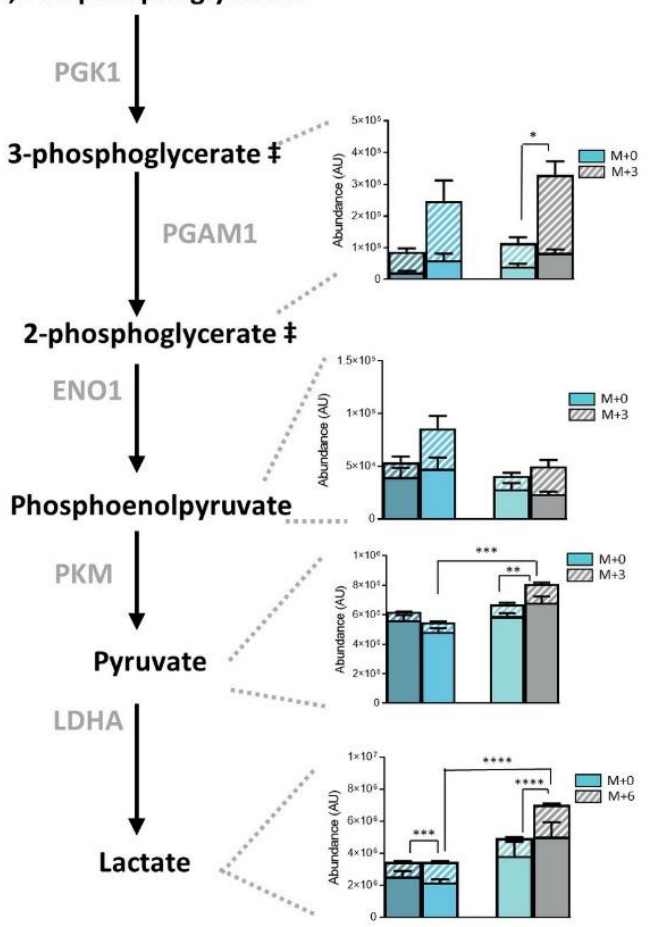


(B)

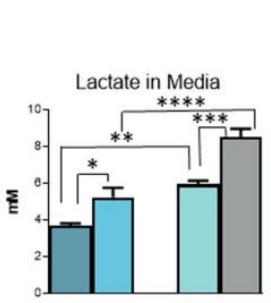

(C)
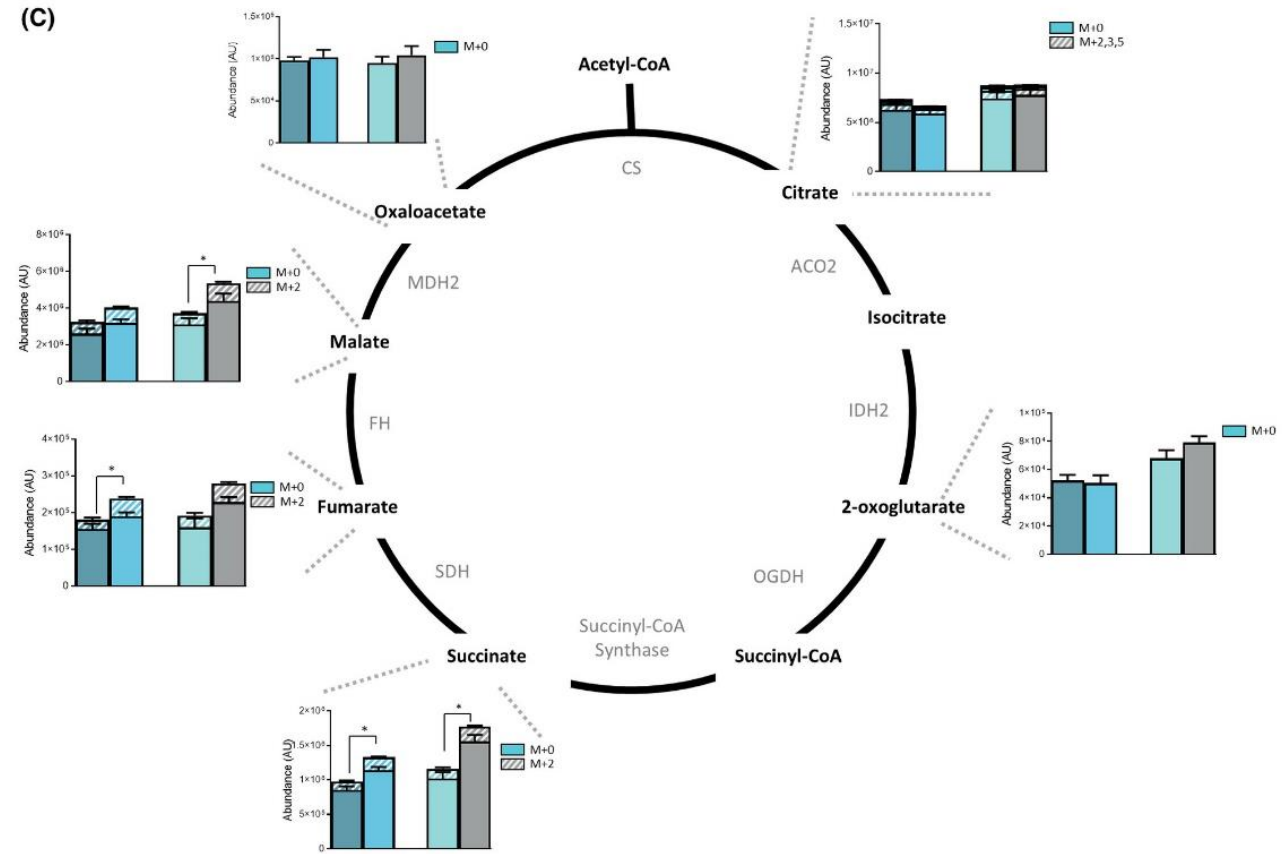

FIGURE 5: ${ }^{13} \mathrm{C}$-glucose carbon tracing confirms increases in glycolysis in epithelial cells lacking Glrx. A, Schematic for glycolysis pathway highlighting metabolites that are differentially regulated 24 hours poststimulation with IL1B and in airway basal cells lacking Glrx. Heavy metabolites are depicted as +3 and +6 and presented with hash marks. Solid color panels represent the unlabeled portion of each metabolite. \#\# Metabolites measured are hexose-6 phosphate (top) and phosphoglycerate (bottom). B, Levels of secreted lactate (in $\mathrm{mM}$ ) in cell culture supernatant of airway basal cells. C, Schematic for metabolites in the TCA cycle. Heavy metabolites are depicted as $+2,+3$, and +5 and presented with hash marks. Solid color panels represent the unlabeled portion of each metabolite. Statistics in this figure represent only the heavy portion of the metabolites, with the exception of lactate in panel B. ${ }^{*} P<.05$, ${ }^{* *}$ $P<.01,{ }^{* * *} P<.001,{ }^{* * *} P<.0001$ (ANOVA). 
(A)
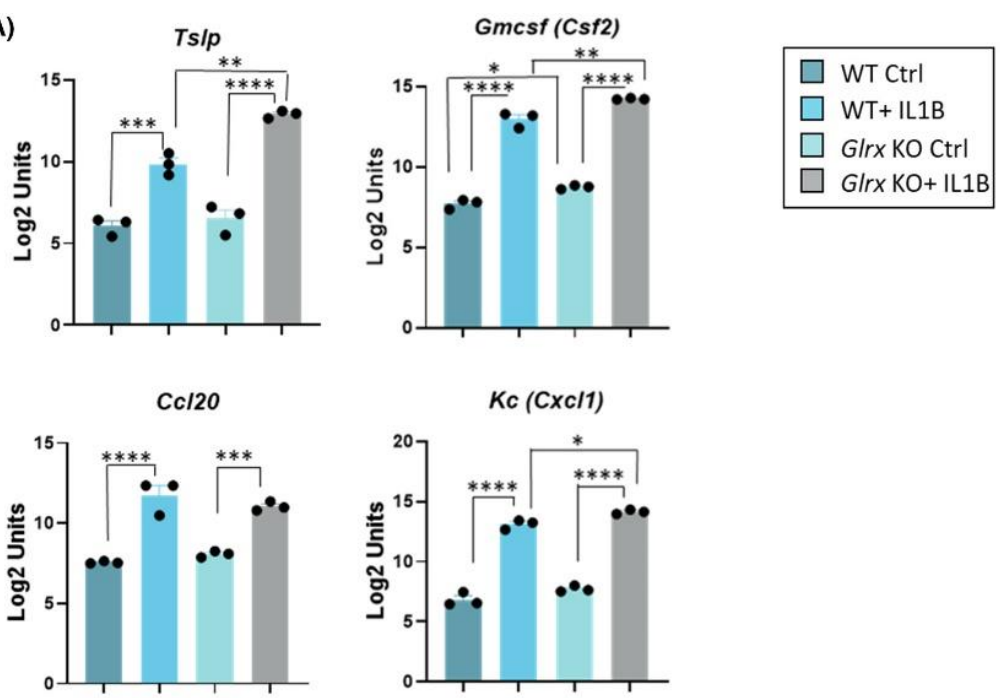

(B)

TSLP
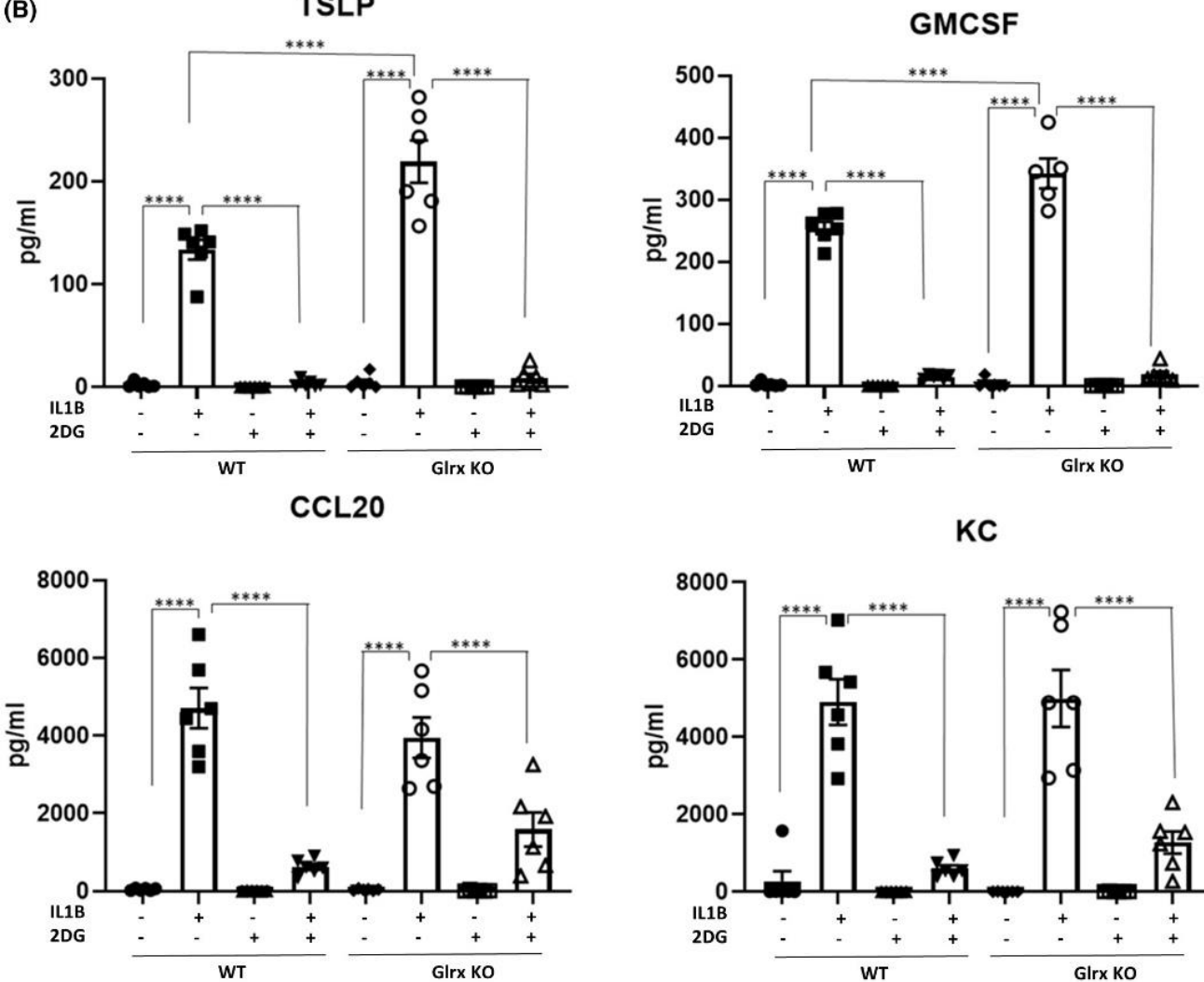

$\mathrm{KC}$

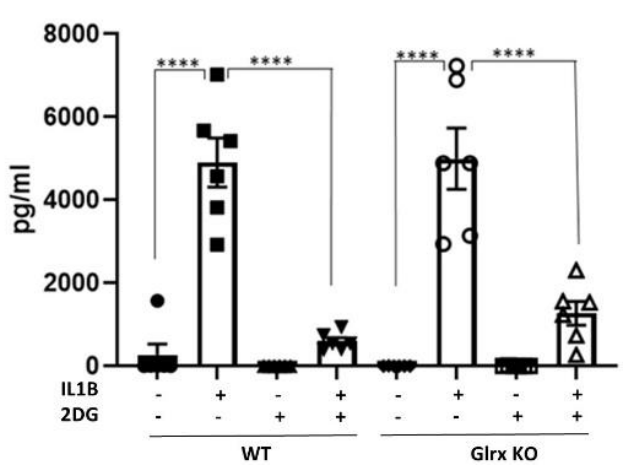

FIGURE 6: Glutaredoxin absence exacerbates pro-inflammatory signaling induced by IL1B in airway basal cells. A, Gene expression of Ts/p, Csf2, Ccl20, and Cxcl1 in WT and Glrx ${ }^{-/}$control airway basal cell or 24 hours after stimulation with IL1B. B, Secreted pro-inflammatory cytokines TSLP, GM-CSF, CCL20, and $\mathrm{KC}$ levels measured in culture media supernatant 24 hours poststimulation with IL1B. A $10 \mathrm{mM}$ of 2-DG was used for 1 hour before IL1B stimulation. 


\section{DISCUSSION}

Airway epithelial cells exert critical effector functions and have considerable plasticity, enabling them to respond to inhaled insults via production of cytokines, growth factors, anti-microbials, surfactants, mucins, etc The extent to which changes in metabolism and redox processes affect epithelial effector function remains incompletely described. Herein, we utilize a multi-omics approach involving $\mathrm{C}^{13}$-glucose tracing, targeted metabolomics, DNA array, and redox proteomics following stimulation with the critical innate immune effector, IL1B, to obtain insights into the pathways governing epithelial cell responses. Furthermore, we conducted these experiments in WT versus Glrx ${ }^{-}$epithelial cells in order to address the extent to which these cellular pathways were affected by glutathionylation chemistry. KEGG pathway analyses demonstrate the modulation of multiple pathways between the different treatment groups, with notable changes in the glycolysis and TCA cycle across the different-omics platforms. Our results show that in the absence of Glrx, IL1B-induced glycolysis is enhanced along with increased secretion of the asthma-relevant cytokine, TSLP. These observations are also supported by the identification of a substantial number of glutathionylated protein targets in the glycolysis pathway and TCA cycle and point to a close interplay between glutathionylation chemistry and glucose metabolism.

Links between glutathione, and glycolysis are well recognized in immune cell and cancer biology. Glutathione primes T-cell metabolism and effector function. $\underline{38}, \underline{39}$ Furthermore, in cancer cells, activation of the pentose phosphate pathway increases NADPH and promotes glutathione redox homeostasis to allow tumor cells to survive in oxidative environments. Interestingly, the pyruvate kinase M2 isoform, which is expressed in glycolytically active cells, can be inactivated via oxidation of key cysteines. $\frac{11}{}$ This event is important in activation of the pentose phosphate pathway and control of glutathione-mediated redox balance.11 PKM was one of the glutathionylation targets identified in the present study, suggesting that its glutathionylation may contribute to the enhanced glycolytic reprogramming induced by IL1B or in Glrx ${ }^{-/}$cells described herein. A recent study from our laboratories demonstrating that a small molecule activator of PKM2 diminished IL1B-induced lactate and TSLP secretion $\underline{34}$ supports a putative scenario wherein glutathionylation of PKM2 is linked to its inactivation and contributes to glycolytic reprogramming and the activation of pro-inflammatory signals.

The findings obtained in epithelial cells that are presented herein are supported by prior studies demonstrating that influenza A virus-infected epithelial cells show activation of numerous metabolic pathways. $\underline{40}$ A role for glycolysis in the control of influenza $A$ infection and replication is plausible based on observations that hypoxia-inducible factor $1 \alpha(\mathrm{HIF}-1 \alpha)$ deficiency in epithelial 
cells decreased glycolysis and promoted autophagy leading to enhanced influenza A virus replication and acute lung injury. 11 Further, HIF-1a-mediated activation of glycolysis in combination with inhibition of prolyl hydroxylases was shown to protect epithelial cells from neutrophil or lipopolysaccharide-induced epithelial cell death and afforded protection from acute lung injury. $\underline{42}$ Interestingly, HIF-1a glutathionylation leads to its stabilization. $\underline{43}$ Although HIF-1 $\alpha$ was not identified in the glutathionylated proteome described herein, future studies will be required to address whether HIF-1 1 -dependent signals contribute to metabolic reconfiguration in epithelial cells exposed to IL1B. Finally, a role for glycolysis and autophagy in epithelial repair was demonstrated in variant Club cells, an airway epithelial progenitor cell population. $\underline{6}$ The authors showed that inhibition of autophagy decreased glucose uptake, and furthermore that glucose deprivation, or glycolysis blockade, decreased the proliferative capacity of these progenitor cells and instead promoted their differentiation into ciliated and secretory cells. $\underline{6}$ These observations point to a critical role of the autophagy and glycolysis pathways in controlling the regenerative/differentiation potential of epithelial cells. The present study utilized airway basal progenitor cells, therefore, additional studies will be required to determine how Glrx and protein glutathionylation control epithelial differentiation.

Finally, it is important to highlight a number of caveats to our work. Following methyl methanethiosulfonate (MMTS) blocking of reduced sulfhydryls, we used wild-type murine GLRX as the catalyst for cysteine derivatization enabling deconvolution of the glutathionylated proteome. This approach differs from the protocols using chemical derivatization strategies that are commonly used in redox proteomics studies. Although we revealed numerous protein targets, the specific cysteines that are glutathionylated and their implications for alterations in structure/function will require additional protocol refinement and follow-up mechanistic studies. Those studies will be required in order to unravel the functional implications of key target cysteines in the glycolysis pathway and how their glutathionylation affects glycolytic reprogramming. An alternative explanation for the findings presented herein could be that the observed changes in glycolysis are not due to glutathionylation of a key glycolysis protein target(s), but instead are the result of a compensatory response resulting from the effects of glutathionylation on other cellular processes, such as ER stress or alterations in mitochondrial function. Furthermore, additional analysis also will be necessary to determine whether changes in glutathionylation are linked to changes in overall target protein abundance. Finally, besides its role as a deglutathionylase, murine GLRX is a dithiol oxidoreductase that can reduce disulfides. We therefore cannot rule out that the protein targets revealed by our screen were strictly glutathionylated. However, validation of selected targets in the glycolysis pathway using a glutathione-specific antibody (Figure 1D) 
support our observations. It also is puzzling that the glutathionylated proteome was not increased in IL1B-stimulated Glr $x^{-/}$cells compared to WT counterparts, which contrasts with the overall GSH Western blot shown in Figure $1 \mathrm{~A}$. The reason for this discrepancy is not clear and will await further analyses related to the efficiency of the GLRX-mediated derivatization. Finally, the experimental design herein represents a snapshot analysis conducted at one time point that does not give insight into temporal relationships between redox and metabolic states that may fluctuate dynamically following IL1B stimulation.

In summary, using a multi-omics platform, we have demonstrated herein that epithelial cells stimulated with IL1B exhibit substantial changes in cellular metabolism which are accentuated in the absence of Glrx. KEGG pathway analyses highlight target enrichments in the TCA cycle and glycolysis pathway. We also show herein that expression of a key asthma cytokine, TSLP, is enhanced in the absence of Glrx and was abrogated using the glycolysis inhibitor 2-DG. Collectively, these observations point to an intricate relationship between glutathionylation chemistry and metabolic alterations within epithelial cells, findings that offer a rationale for utilizing the GLRX/glutathionylation axis to target metabolic reconfiguration for therapeutic purposes. 


\section{REFERNCES:}

1. DeBerardinis RJ, Chandel NS. Fundamentals of cancer metabolism. Sci Adv. 2016;2(5):e1600200.

2. Everts $B$, Amiel E, Huang S-C, et al. TLR-driven early glycolytic reprogramming via the kinases TBK1-IKKE supports the anabolic demands of dendritic cell activation. Nat Immunol. 2014;15(4):323-332.

3. O'Neill LA, Pearce EJ. Immunometabolism governs dendritic cell and macrophage function. J Exp Med. 2016;213(1):15-23.

4. Tannahill GM, Curtis AM, Adamik J, et al. Succinate is an inflammatory signal that induces IL-16 through

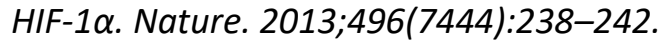

5. Angiari S, Runtsch MC, Sutton $C E$, et al. Pharmacological activation of pyruvate kinase M2 inhibits CD4(+) T cell pathogenicity and suppresses autoimmunity. Cell Metab. 2020;31(2):391-405.e8.

6. Davies DE. Epithelial barrier function and immunity in asthma. Ann Am Thorac Soc. 2014;11(Suppl 5):S244-S251.

7. Hartl D, Tirouvanziam R, Laval J, et al. Innate immunity of the lung: from basic mechanisms to translational medicine. J Innate Immun. 2018;10(5-6):487-501.

8. Qian XI, Aboushousha R, van de Wetering C, et al. IL-1/inhibitory $\kappa B$ kinase $\varepsilon$-induced glycolysis augment epithelial effector function and promote allergic airways disease. J Allergy Clin Immunol. 2018;142(2):435450.e10.

9. Li K, Li M, Li W, et al. Airway epithelial regeneration requires autophagy and glucose metabolism. Cell Death Dis. 2019;10(12):875.

10. Harris I, Treloar A, Inoue S, et al. Glutathione and thioredoxin antioxidant pathways synergize to drive cancer initiation and progression. Cancer Cell. 2015;27(2):211-222.

11. Anastasiou D, Poulogiannis G, Asara JM, et al. Inhibition of pyruvate kinase $M 2$ by reactive oxygen species contributes to cellular antioxidant responses. Science. 2011;334(6060):1278-1283.

12. Mohr S, Stamler JS, Brüne B. Posttranslational modification of glyceraldehyde-3-phosphate dehydrogenase by S-nitrosylation and subsequent NADH attachment. J Biol Chem. 1996;271(8):42094214.

13. Reisz JA, Wither MJ, Dzieciatkowska M, et al. Oxidative modifications of glyceraldehyde 3-phosphate dehydrogenase regulate metabolic reprogramming of stored red blood cells. Blood. 2016;128(12):e32e42.

14. Seo $M$, Lee YH. PFKFB3 regulates oxidative stress homeostasis via its S-glutathionylation in cancer. J Mol Biol. 2014;426(4):830-842.

15. Zaffagnini M, Michelet L, Sciabolini $C$, et al. High-resolution crystal structure and redox properties of chloroplastic triosephosphate isomerase from Chlamydomonas reinhardtii. Mol Plant. 2014;7(1):101-120.

16. Fratelli $M$, Demol $H$, Puype $M$, et al. Identification by redox proteomics of glutathionylated proteins in oxidatively stressed human T lymphocytes. Proc Natl Acad Sci USA. 2002;99(6):3505-3510.

17. Sies $H$, Jones DP. Reactive oxygen species (ROS) as pleiotropic physiological signalling agents. Nat Rev Mol Cell Biol. 2020;21(7):363-383. 
18. Janssen-Heininger $Y$, Reynaert NL, van $\operatorname{der}$ Vliet $A$, Anathy $V$. Endoplasmic reticulum stress and glutathione therapeutics in chronic lung diseases. Redox Biol. 2020;33:101516.

19. Chia SB, Elko EA, Aboushousha R, et al. Dysregulation of the glutaredoxin/S-glutathionylation redox axis in lung diseases. Am J Physiol Cell Physiol. 2020;318(2):C304-C327.

20. Li $Q$, Harraz MM, Zhou W, et al. Nox2 and Rac1 regulate H2O2-dependent recruitment of TRAF6 to endosomal interleukin-1 receptor complexes. Mol Cell Biol. 2006;26(1):140-154.

21. Oakley FD, Smith RL, Engelhardt JF. Lipid rafts and caveolin-1 coordinate interleukin-1beta (IL-1beta)dependent activation of NFkappaB by controlling endocytosis of Nox2 and IL-1beta receptor 1 from the plasma membrane. J Biol Chem. 2009;284(48):33255-33264.

22. Wu R, Smith D. Continuous multiplication of rabbit tracheal epithelial cells in a defined, hormonesupplemented medium. In Vitro. 1982;18(9):800-812.

23. Alcorn JF, Guala AS, van der Velden J, et al. Jun N-terminal kinase 1 regulates epithelial-tomesenchymal transition induced by TGF-beta1. J Cell Sci. 2008;121(Pt 7):1036-1045.

24. Nemkov T, D'Alessandro A, Hansen KC. Three-minute method for amino acid analysis by UHPLC and high-resolution quadrupole orbitrap mass spectrometry. Amino Acids. 2015;47(11):2345-2357.

25. Nemkov T, Reisz JA, Gehrke S, Hansen KC, D'Alessandro A. High-throughput metabolomics: isocratic and gradient mass spectrometry-based methods. Methods Mol Biol. 2019;1978:13-26.

26. Gehrke S, Rice S, Stefanoni D, et al. Red Blood cell metabolic responses to torpor and arousal in the hibernator arctic ground squirrel. J Proteome Res. 2019;18(4):1827-1841.

27. Nemkov T, Hansen KC, D'Alessandro A. A three-minute method for high-throughput quantitative metabolomics and quantitative tracing experiments of central carbon and nitrogen pathways. Rapid Commun Mass Spectrom. 2017;31(8):663-673.

28. r-project. R: A Language and Environment for Statistical Computing. 2020. June 22. Available from: https://www.r-project.org/. Accessed 25 June, 2020.

29. Gu Z, Eils R, Schlesner M. Complex heatmaps reveal patterns and correlations in multidimensional genomic data. Bioinformatics. 2016;32(18):2847-2849.

30. Huber W, Carey VJ, Gentleman R, et al. Orchestrating high-throughput genomic analysis with bioconductor. Nat Methods. 2015;12(2):115-121.

31. MacDonald JW. affycoretools: Functions Useful for those doing Repetitive Analyses with Affymetrix GeneChips. 2020.29 .2 Available from: https://www.bioconductor.org/packages/release/bioc/html/affycoretools.html.

32. Ritchie ME, Phipson B, Wu DI, et al. limma powers differential expression analyses for RNA-sequencing and microarray studies. Nucleic Acids Res. 2015;43(7):e47.

33. Anathy V, Lahue KG, Chapman DG, et al. Reducing protein oxidation reverses lung fibrosis. Nat Med. 2018;24(8):1128-1135.

34. van de Wetering C, Aboushousha R, Manuel AM, et al. Pyruvate kinase M2 promotes expression of proinflammatory mediators in house dust mite-induced allergic airways disease. J Immunol. 2020;204(4):763-774. 
35. Kanehisa M, Goto S. KEGG: kyoto encyclopedia of genes and genomes. Nucleic Acids Res. 2000;28(1):27-30.

36. Gauvreau GM, O'Byrne PM, Boulet L-P, et al. Effects of an anti-TSLP antibody on allergen-induced asthmatic responses. N Engl J Med. 2014;370(22):2102-2110.

37. Corren J, Parnes JR, Wang L, et al. Tezepelumab in Adults With Uncontrolled Asthma. N Engl J Med. 2017;377(10):936-946.

38. Mak TW, Grusdat M, Duncan GS, et al. Glutathione primes $T$ cell metabolism for inflammation. Immunity. 2017;46(4):675-689.

39. Kurniawan H, Franchina DG, Guerra L, et al. Glutathione restricts serine metabolism to preserve regulatory T cell function. Cell Metab. 2020;31(5):920-936.e7.

40. Tian X, Zhang K, Min J, et al. Metabolomic analysis of influenza A virus A/WSN/1933 (H1N1) infected A549 cells during first cycle of viral replication. Viruses. 2019;11(11):1007.

41. Zhao C, Chen J, Cheng L, et al. Deficiency of HIF-1 $\alpha$ enhances influenza A virus replication by promoting autophagy in alveolar type II epithelial cells. Emerg Microbes Infect. 2020;9(1):691-706.

42. Tojo K, Tamada N, Nagamine $Y$, et al. Enhancement of glycolysis by inhibition of oxygen-sensing prolyl hydroxylases protects alveolar epithelial cells from acute lung injury. FASEB J. 2018;32(4):2258-2268.

43. Watanabe $Y$, Murdoch CE, Sano S, et al. Glutathione adducts induced by ischemia and deletion of glutaredoxin-1 stabilize HIF-1 $\alpha$ and improve limb revascularization. Proc NatI Acad Sci USA. 2016;113(21):6011-6016. 


\section{SUPPLEMENTARY FIGURE 1:}

A.

\section{List of glutathionylated proteins}

\begin{tabular}{|c|c|c|c|}
\hline WT Ctrl & WT+ IL1B & Glrx KO Ctrl & Glrx KO+ IL1B \\
\hline $\begin{array}{l}\text { MYH14, ANXA6, } \\
\text { LGALS3, DLD, } \\
\text { TUBA1B, PLS3, } \\
\text { UBE2L3, GNB2, } \\
\text { KRT79, MYO1D, } \\
\text { SELENBP1, GSTO1, } \\
\text { GLRX, ACTB, PKM, } \\
\text { GAPDH, ANXA1, } \\
\text { ANXA2, EEF1A1, } \\
\text { MYH9, ENO1, TKT, } \\
\text { CBR2, LDHA, } \\
\text { CYP2F2, ALDH1A1, } \\
\text { VCP, PGD, PGK1, } \\
\text { ALDH3A1, PRDX1, } \\
\text { MDH2, PRSS1, } \\
\text { TPI1, ESD, KRT1, } \\
\text { KRT10, EEF2, } \\
\text { FLNB, KRT78, } \\
\text { ALDOA, } \\
\text { HSP9OAB1, EEF1G, } \\
\text { ETFB, PCBP1, } \\
\text { GSTM2, ALB, } \\
\text { KXD1, SFN, PPIA, } \\
\text { CSTB, RPLP0, } \\
\text { CLIC1, RNH11, } \\
\text { FLNA, ERO1A, } \\
\text { CAST, VDAC3, } \\
\text { FASN, CS, } \\
\text { YWHAG, YWHAQ, } \\
\text { HNRNPU, IDH1, } \\
\text { HNRNPM, RPL12, } \\
\text { ANXA4, KRT5, } \\
\text { CTNND1, WDR1, } \\
\text { HNRNPK, TAGLN2, } \\
\text { CCT4, PGAM1, } \\
\text { S100A6, CLTC, } \\
\text { DSTN, GSN, } \\
\text { SEPTIN-9, ATP5A1, } \\
\text { KRT2, IMMT, } \\
\text { SQOR, PRDX6, } \\
\text { SLC25A5, OTUB1, } \\
\text { TUBB5, VILL, } \\
\text { IDH2, CAP1, } \\
\text { GPD2, AHNAK, } \\
\text { MYOF, PYGB, } \\
\text { ATP1A1, KRT76, } \\
\text { GSTP1, GSTM1, } \\
\text { GOT2, AHSA1, } \\
\text { ETFA, PCBP2 }\end{array}$ & 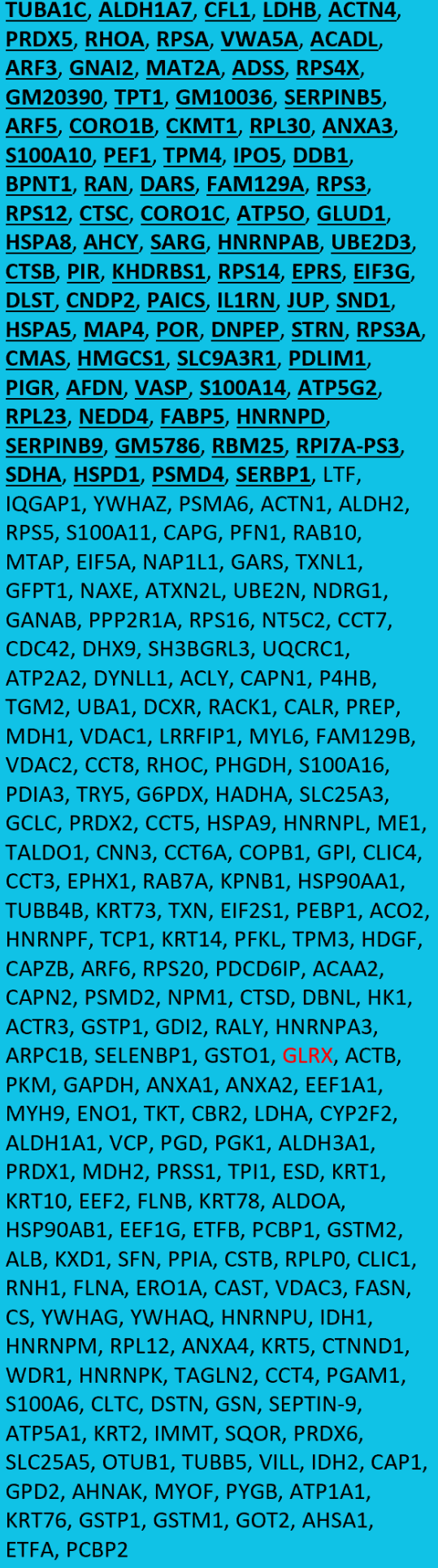 & $\begin{array}{l}\text { ACTC1, NIPSNAP3A, EIF4B, } \\
\text { LTA4H, ESPN, LASP1, } \\
\text { CTNNA1, ERP44, NME1, } \\
\text { CTSH, PARK7, CTNNB1, } \\
\text { PSMD1, HIST2H3C1, P4HB, } \\
\text { TGM2, UBA1, DCXR, } \\
\text { RACK1, CALR, PREP, MDH1, } \\
\text { VDAC1, LRRFIP1, MYL6, } \\
\text { FAM129B, VDAC2, CCT8, } \\
\text { RHOC, PHGDH, S100A16, } \\
\text { PDIA3, TRY5, G6PDX, } \\
\text { HADHA, SLC25A3, GCLC, } \\
\text { PRDX2, CCT5, HSPA9, } \\
\text { HNRNPL, ME1, TALDO1, } \\
\text { CNN3, CCT6A, COPB1, GPI, } \\
\text { CLIC4, CCT3, EPHX1, } \\
\text { RAB7A, KPNB1, HSP90AA1, } \\
\text { TUBB4B, KRT73, TXN, } \\
\text { EIF2S1, PEBP1, ACO2, } \\
\text { HNRNPF, TCP1, KRT14, } \\
\text { PFKL, TPM3, HDGF, CAPZB, } \\
\text { ARF6, RPS20, PDCD6IP, } \\
\text { ACAA2, CAPN2, PSMD2, } \\
\text { NPM1, CTSD, DBNL, HK1, } \\
\text { ACTR3, GSTP1, GDI2, RALY, } \\
\text { HNRNPA3, ARPC1B, } \\
\text { TUBA1B, PLS3, UBE2L3, } \\
\text { GNB2, KRT79, MYO1D, } \\
\text { SELENBP1, GSTO1, GLRX, } \\
\text { ACTB, PKM, GAPDH, } \\
\text { ANXA1, ANXA2, EEF1A1, } \\
\text { MYH9, ENO1, TKT, CBR2, } \\
\text { LDHA, CYP2F2, ALDH1A1, } \\
\text { VCP, PGD, PGK1, ALDH3A1, } \\
\text { PRDX1, MDH2, PRSS1, TPI1, } \\
\text { ESD, KRT1, KRT10, EEF2, } \\
\text { FLNB, KRT78, ALDOA, } \\
\text { HSP90AB1, EEF1G, ETFB, } \\
\text { PCBP1, GSTM2, ALB, KXD1, } \\
\text { SFN, PPIA, CSTB, RPLP0, } \\
\text { CLIC1, RNH1, FLNA, ERO1A, } \\
\text { CAST, VDAC3, FASN, CS, } \\
\text { YWHAG, YWHAQ, } \\
\text { HNRNPU, IDH1, HNRNPM, } \\
\text { RPL12, ANXA4, KRT5, } \\
\text { CTNND1, WDR1, HNRNPK, } \\
\text { TAGLN2, CCT4, PGAM1, } \\
\text { M100A6, CLTC, DSTN, GSN, , ATP5A1, KRT2, } \\
\text { KRT76, GSTP1 }\end{array}$ & $\begin{array}{l}\text { CNN2, DNM2, PPP1CC, PCLO, } \\
\text { MIER1, USP14, OGDH, MYO1C, } \\
\text { HNRNPC, CCT2, PPP2CB, } \\
\text { BAG3, XPO1, RPL5, LMNB1, } \\
\text { AP2M1, SEC24C, RBBP4, NARS, } \\
\text { LTF, IQGAP1, YWHAZ, PSMA6, } \\
\text { ACTN1, ALDH2, RPS5, S100A11, } \\
\text { CAPG, PFN1, RAB10, MTAP, } \\
\text { EIF5A, NAP1L1, GARS, TXNL1, } \\
\text { GFPT1, NAXE, ATXN2L, UBE2N, } \\
\text { NDRG1, GANAB, PPP2R1A, } \\
\text { RPS16, NT5C2, CCT7, CDC42, } \\
\text { DHX9, SH3BGRL3, UQCRC1, } \\
\text { ATP2A2, DYNLL1, ACLY, CAPN1, } \\
\text { PRDX6, SLC25A5, OTUB1, } \\
\text { TUBB5, VILL, IDH2, CAP1, } \\
\text { GPD2, CTSH, PARK7, CTNNB1, } \\
\text { PSMD1, HIST2H3C1, KRT79, } \\
\text { MYO1D, P4HB, TGM2, UBA1, } \\
\text { DCXR, RACK1, CALR, PREP, } \\
\text { MDH1, VDAC1, LRRFIP1, MYL6, } \\
\text { FAM129B, VDAC2, CCT8, } \\
\text { RHOC, PHGDH, S100A16, } \\
\text { PDIA3, TRY5, G6PDX, HADHA, } \\
\text { SLC25A3, GCLC, PRDX2, CCT5, } \\
\text { HSPA9, HNRNPL, ME1, } \\
\text { TALDO1, CNN3, CCT6A, COPB1, } \\
\text { GPI, CLIC4, CCT3, EPHX1, } \\
\text { RAB7A, KPNB1, SELENBP1, } \\
\text { GSTO1, GLRX, ACTB, PKM, } \\
\text { GAPDH, ANXA1, ANXA2, } \\
\text { EEF1A1, MYH9, ENO1, TKY, } \\
\text { CBR2, LDHA, CYP2F2, } \\
\text { ALDH1A1, VCP, PGD, PGK1, } \\
\text { ALDH3A1, PRDX1, MDH2, } \\
\text { PRSS1, TPI1, ESD, KRT1, KRT10, } \\
\text { EEF2, FLNB, KRT78, ALDOA, } \\
\text { HSP90AB1, EEF1G, ETFB, } \\
\text { PCBP1, GSTM2, ALB, KXD1, } \\
\text { SFN, PPIA, CSTB, RPLPO, CLIC1, } \\
\text { RNH1, FLNA, ERO1A, CAST, } \\
\text { VDAC3, FASN, CS, YWHAG, } \\
\text { YWHAQ, HNRNPU, IDH1, } \\
\text { HNRNPM, RPL12, ANXA4, } \\
\text { KRT5, CTNND1, WDR1, } \\
\text { HNRNPK, TAGLN2, CCT4, } \\
\text { PGAM1, S100A6, CLTC, DSTN, } \\
\text { GSN, SEPTIN-9, ATP5A1, KRT2, } \\
\text { IMMT, SQOR }\end{array}$ \\
\hline
\end{tabular}


B.
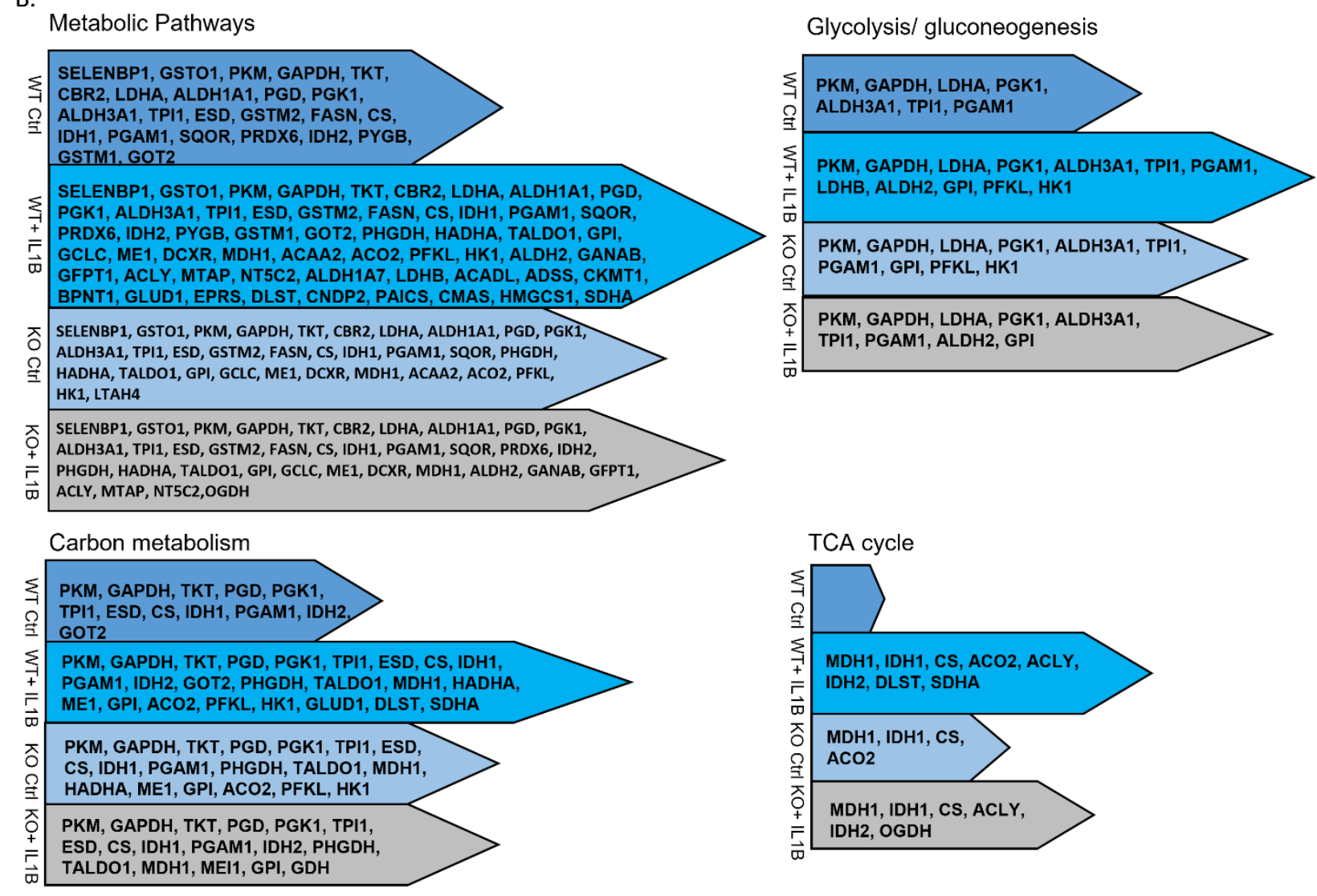

Supplementary Figure 1: S-glutathionylated proteins identified via redox-Proteomic analysis of WT or Glrx ${ }^{-/}$airway basal cells under base line conditions or in response to stimulation with IL1BA: Glutathionylated proteins appearing in control or IL1B-stimulated WT or Glr-'-airway basal cells. Unique proteins detected in each group are in bold and underlined. Exogenously added GLRX identified in the four groups is highlighted in red. B: Identity of glutathionylated proteins hits appearing in select KEGG pathways identified in Figure1C in each of the four treatment groups. 


\begin{tabular}{|c|c|c|c|}
\hline WT Ctrl vs KO Ctrl & WT Ctrl vs WT+IL1B & WT IL1B vs KO+IL1B & KO Ctrl vs KO+IL1B \\
\hline $\begin{array}{l}\text { Aminoacyl t-RNA } \\
\text { biosynthesis }\end{array}$ & $\begin{array}{l}\text { Aminoacyl t-RNA } \\
\text { biosynthesis }\end{array}$ & $\begin{array}{l}\text { Aminoacyl t-RNA } \\
\text { biosynthesis }\end{array}$ & $\begin{array}{l}\text { Aminoacyl t-RNA } \\
\text { biosynthesis }\end{array}$ \\
\hline \multirow[t]{8}{*}{$\begin{array}{l}\text { Arginine and proline } \\
\text { metabolism }\end{array}$} & & $\begin{array}{l}\text { Arginine and proline } \\
\text { metabolism }\end{array}$ & \\
\hline & $\begin{array}{l}\text { Alanine, Aspartate and } \\
\text { Glutamate metabolism }\end{array}$ & $\begin{array}{l}\text { Alanine, Aspartate and } \\
\text { Glutamate metabolism }\end{array}$ & $\begin{array}{l}\text { Alanine, Aspartate and } \\
\text { Glutamate metabolism }\end{array}$ \\
\hline & & & \\
\hline & Arginine biosynthesis & Arginine biosynthesis & \\
\hline & $\begin{array}{l}\text { Phenylalanine, tyrosine\& } \\
\text { Tryptophan biosynthesis }\end{array}$ & & $\begin{array}{l}\text { Phenylalanine, tyrosine } \& \\
\text { Tryptophan biosynthesis }\end{array}$ \\
\hline & \multirow{3}{*}{$\begin{array}{l}\text { Glutathione metabolism } \\
\text { Valine, leucine\& } \\
\text { Isoleucine biosynthesis }\end{array}$} & & \\
\hline & & Citrate (TCA) cycle & Citrate (TCA) cycle \\
\hline & & & $\begin{array}{c}\text { Glyoxylate\& } \\
\text { Dicarboxylate metabolism }\end{array}$ \\
\hline
\end{tabular}

Supplementary Figure 2: Pathway analysis for the differentially regulated metabolites shown in Figure 2: Shown are comparisons between WT ctrl to Glrx ${ }^{-/}$ctrl, WT ctrl to WT+ IL1B, WT+ IL1B to $\mathrm{Glrx}^{-{ }^{-}}+$IL1B and Glrx ${ }^{-/-}$ctrl to Glrx ${ }^{-/}+$IL1B groups. Metaboanalyst was used to generate the list with FDR value of $<0.05$ as the cut off. 


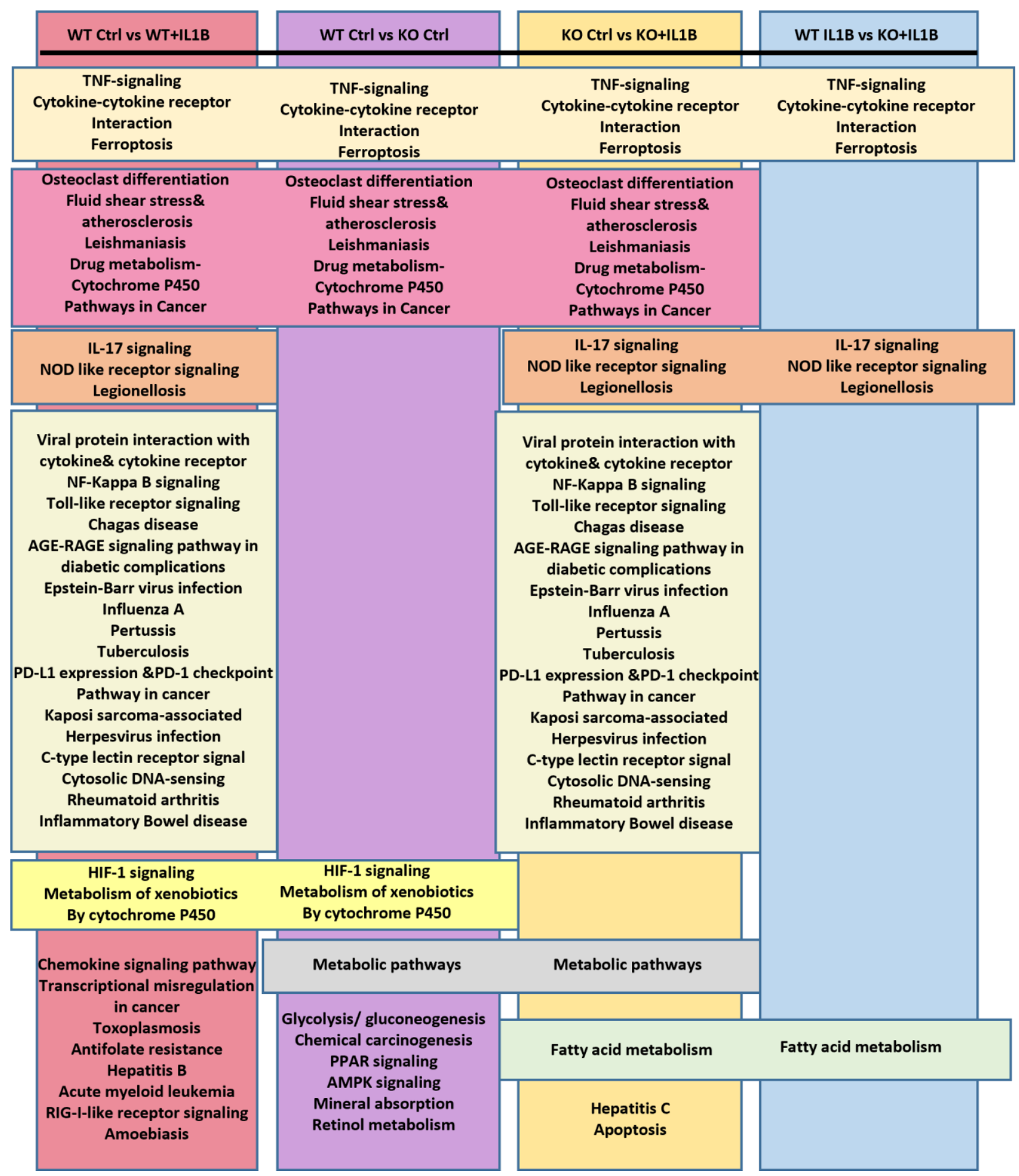

Supplementary Figure 3: KEGG pathway analysis for the differentially regulated genes shown in Figure 3: Shown are comparisons between WT ctrl to Glrx ${ }^{-1}$ ctrl, WT ctrl to WT+ IL1B, WT+ IL1B to Glrx ${ }^{-1}+$ IL1B and Glrx ${ }^{-1}$ ctrl to Glrx ${ }^{-1}+$ IL1B groups. Log fold change of 1 was chosen as a cut off value for genes included in the analysis. 


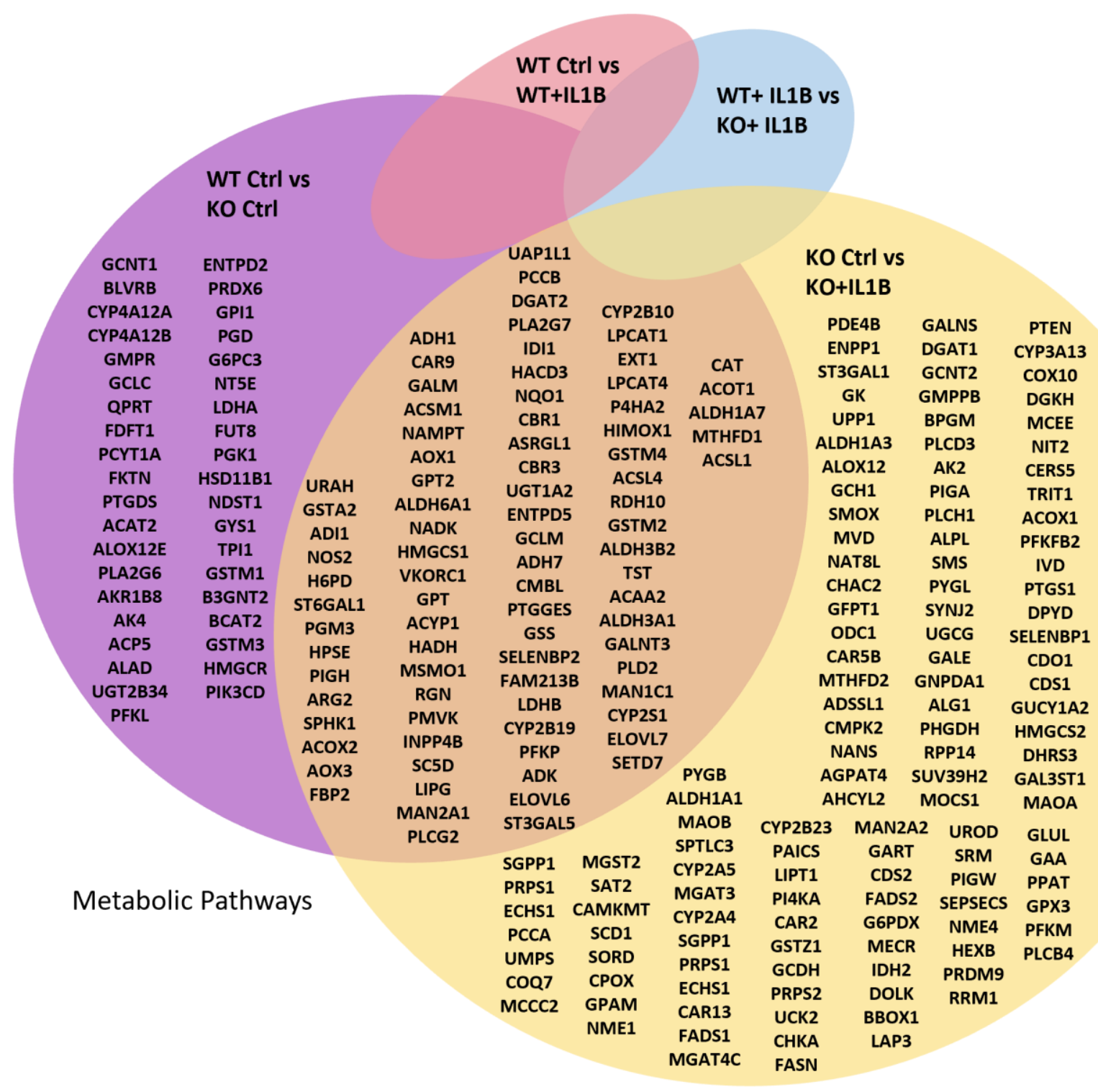

Supplementary Figure 4: Venn diagrams highlighting unique and overlapping genes in the metabolic pathways identified from KEGG analysis shown in Supplementary Figure 3. 
A.
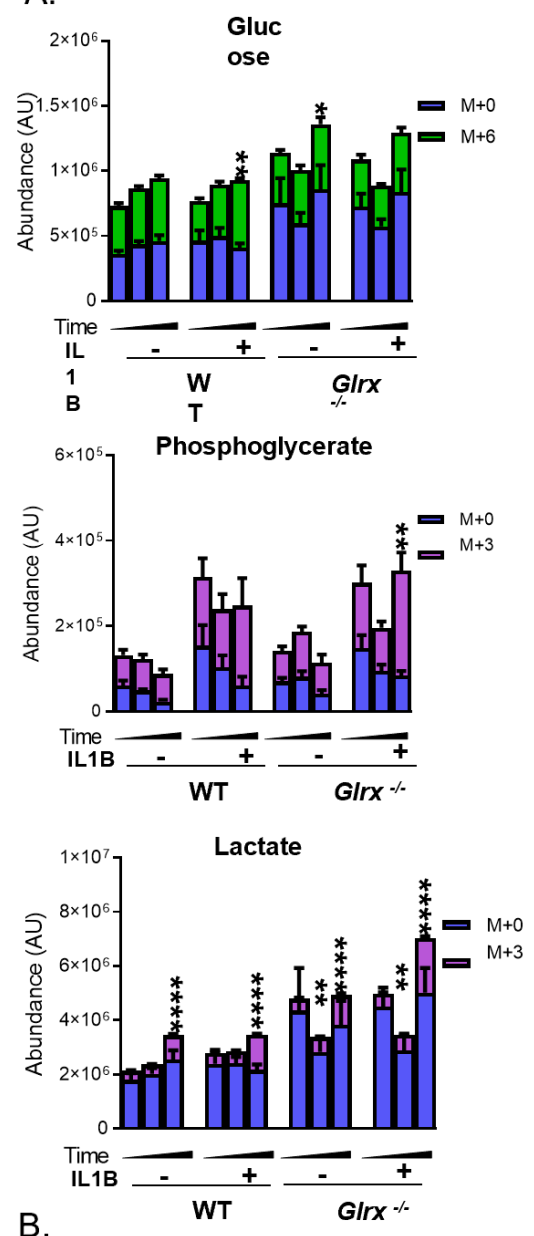

B.
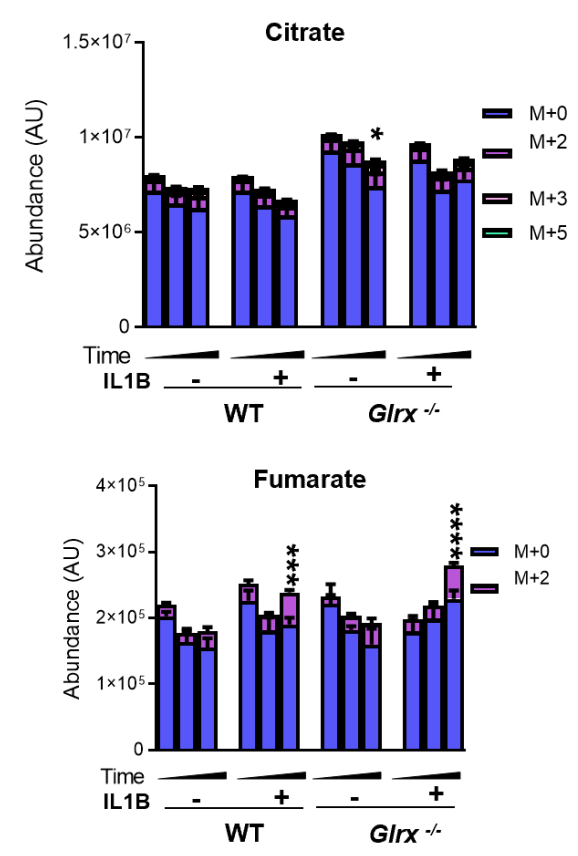
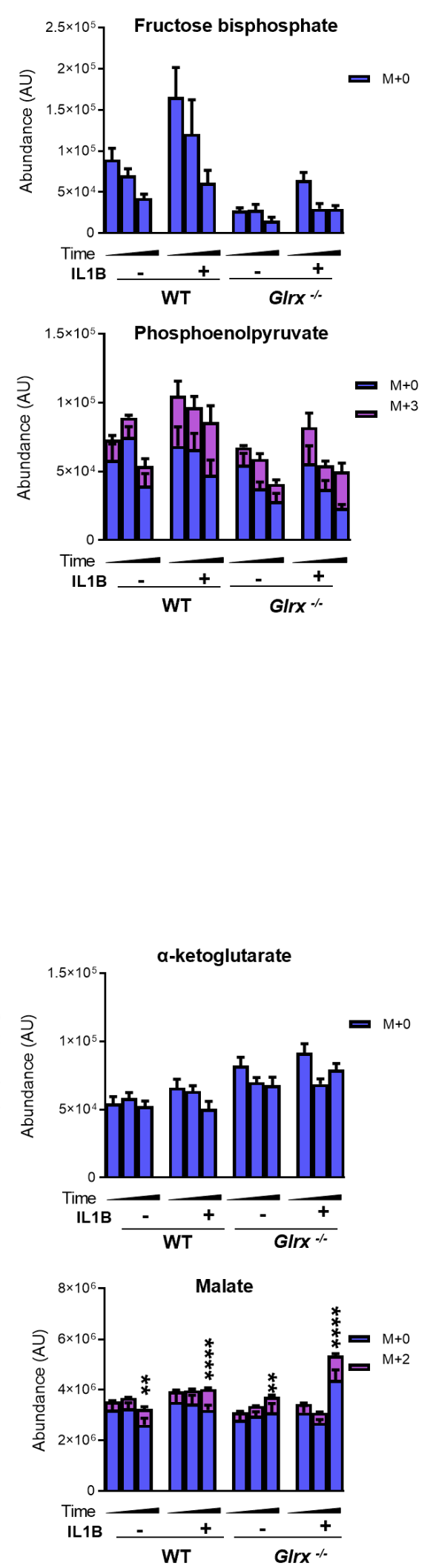
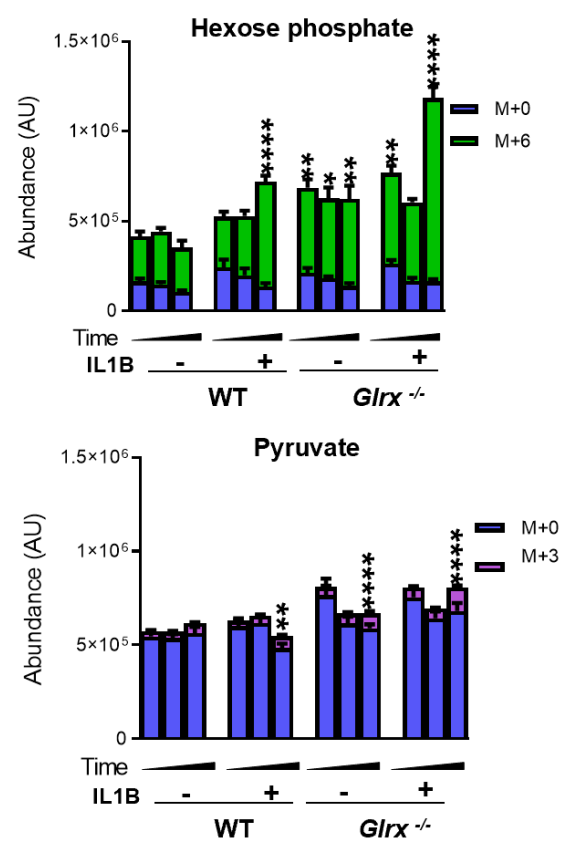
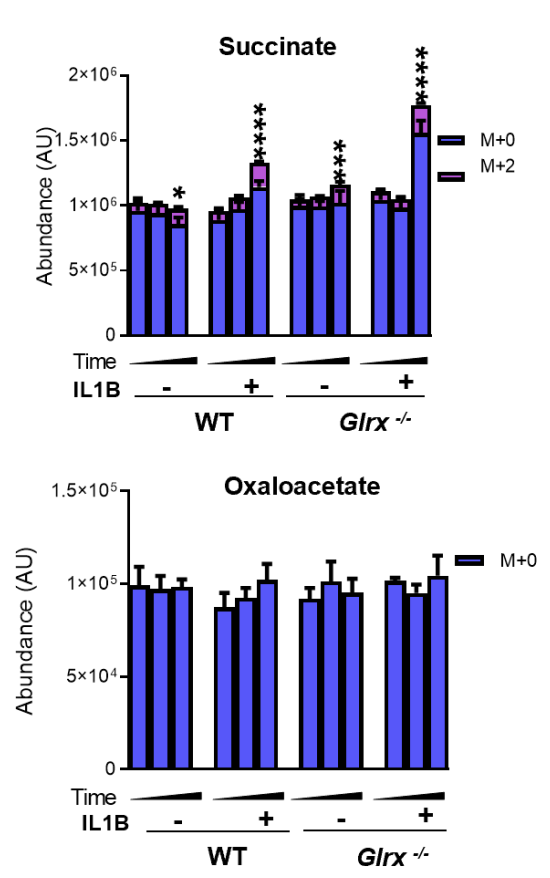
Supplementary Figure 5: ${ }^{13} \mathrm{C}$ carbon tracing metabolites from epithelial cells treated with IL1B for 2 (left), 6 (middle) and 24 (right) hours. M+0 depicts the unlabeled, while $M+2,3,5$ and 6 depict the labeled portion of the metabolites in the glycolysis pathway $(A)$ and the TCA cycle (B). ${ }^{*} p<0.05,{ }^{* *} p<0.01,{ }^{* * *} p<0.001,{ }^{* * *} p<0.0001$ (ANOVA). Statistical analyses in this represent only the heavy portion of the metabolites. All groups were compared to WT control at 2 hours. 


\section{CHAPTER 6:}

Glutaredoxin-1 controls glutathione levels via deglutathionylation of the ovarian tumor deubiquitinase, OTUB1 and stabilization of system $x_{c^{-}}$

Aboushousha R., van der Velden JL, Peng Z, Chia SB, MacPherson MB, Erickson C, Reisz JA, D’Alessandro A, Wouters EFM, Reynaert NL, Janssen-Heininger YMW.

Manuscript in progress 
CHAPTER 7: GENERAL DISCUSSION AND FUTURE PERSPECTIVES 
In the early 1920s, the German biochemist Otto Warburg made a groundbreaking discovery that completely changed our understanding about cells' metabolic state/preferences. In his work, Warburg explained that tumor cells prefer to use glycolysis for glucose metabolism, rather than the more efficient ATP generating mitochondrial oxidative phosphorylation, even in oxygen-rich conditions [1]. Since then, multiple lines of scientific evidence emerged proving that it is not only the glucose consumption, but instead, the entire metabolic network that undergoes extensive reprogramming to redirect the flow and flux of several nutrients to meet the tumor's energy and macromolecules demand. Recently, several studies confirmed that metabolic reprogramming is not confined to cancer cells alone, and that several other cell types exhibit metabolic reprogramming including proliferating cells, T-cells and myofibroblasts [2-5]. In addition, multiple cellular processes including proliferation, differentiation, autophagy, apoptosis and inflammatory responses have been described to be modulated through metabolic reprogramming [6-9]. Glycolysis, a great determinant of cell's metabolic state, generates not only ATP but also glycolytic intermediates necessary for the biosynthesis of nucleic acids, proteins, lipids and for generating NADPH reducing equivalents (through the PPP) required for redox balance [2]. Interestingly, glycolytic reprogramming and redox imbalance started to emerge as contributors to multiple diseases such as cancer, neuro/muscular degenerative and several inflammatory disorders, however the exact molecular mechanisms governing these processes are not yet fully clear [10-13]. In this thesis, we aimed at studying the connection between metabolic reprogramming, notably glycolysis, and redox homeostasis as well as the possible impact of their crosstalk on inflammatory signaling. We went beyond studying the molecular signaling in cells to examine involvement of our findings in an asthma model. The chronic inflammatory state induced by airway epithelial cell activation and/or damage, and the flux of inflammatory cells in asthma, are usually accompanied by metabolic changes as well as shifts in redox homeostasis [14-17], however the mechanistic details are yet to be unraveled.

\section{Glycolytic reprogramming and IL1B in asthma: new paradigm and asthma biomarkers}

As explained earlier in the introduction, asthma is a heterogenous disease with several phenotypes (observable characteristics), endotypes (underlying mechanisms) and a big overlap between them [18]. This makes it extremely difficult to subgroup and categorize all patients using the traditional parameters [18]. A substantial subgroup of patients, mostly with neutrophilic influx, suffer from severe disease with less response to therapy. Therefore, there is a dire need of identifying new biomarkers to help characterize this subset of patients. A better understanding of 
the mechanisms underlying asthma pathophysiology is likewise needed to help in designing new therapeutics. Glycolytic reprogramming has been described to contribute to multiple diseases, including Alzheimer's and Parkinson's disease, and Amyotrophic Lateral Sclerosis [19]. In fact, in these diseases, aberrant glycolysis has been proposed as a biomarker to the different disease stages [19]. In addition, glycolysis- related genes also serve as prognostic tools in some cancer subtypes [20]. In this thesis we highlight increases in glycolysis as a critical feature of asthma pathophysiology (chapter 3,4 ). Work by other groups also support that aerobic glycolysis can be involved in asthma by occurring in different cells and affecting different steps in its pathogenic process [21-24]. For instance, Ostroukhova et al, showed that aerobic glycolysis is enhanced in asthma due to its effect on T cell activation. As anticipated, they showed that blocking glycolysis using 2DG, a hexokinase inhibitor, attenuates T cell proliferation and cytokine production [21]. Interestingly, our data also show that inhibition of glycolysis (by 2DG and oxamate) significantly dropped levels of TSLP, GM-CSF, CCL20 and KC, cytokines important in asthma pathogenesis. Together, these findings position aberrant changes of the glycolysis pathway on the map of asthma pathogenesis.

Moreover, we herein demonstrate that IL1B is the driving signal for the increased glycolysis in HDM-induced allergic airways disease and in lung epithelial cells (chapter 3, 4, 5). The pleiotropic cytokine IL1B has been implicated in multiple diseases including diabetes [25], cancer [26], as well as several autoimmune and metabolic syndromes [27]. In addition, dysregulation in IL1B synthesis and excess of its release have been found to contribute to the pathogenesis of inflammatory bowel disease, psoriasis and rheumatoid arthritis [28]. In the lungs, IL1B is present in the airways of patients with asthma, and the cytokine has been shown to play a role in the early phase of both the inflammatory events as well as the altered airway responsiveness in asthmatics [29, 30]. Moreover, IL1B was shown to modulate airway constriction and relaxation in atopic asthmatics by its direct action on airways smooth muscle [31]. In response to inhaled allergens, IL1B instructs dendritic cells to induce the Th2 responses and directly promotes activation of Th2 cells [32]. Interestingly, and in agreement with our data, IL1B has been shown to be required for the induction of chemotactic factors, IL-33 and Muc5ac in a viral-induced asthma model, pointing at its role in neutrophilic and Th2 inflammatory response [33]. Moreover, the inflammatory state in most asthmatic patients is driven by allergen-induced Th2 response and is accompanied by elevated eosinophil counts. This group of patients luckily benefits from corticosteroid, the first line treatment in asthma patients [34]. However, a substantial subpopulation of patients exhibits a similar inflammatory response but lack steroid responsiveness, or instead display neutrophilic inflammation [34]. These patients usually suffer 
from severe uncontrolled disease. Recently, it has been shown that IL1 signaling increases glycolysis during differentiation of Th17 cells, a subset of T cells associated with severe asthma and responsible for initiating inflammatory response in autoimmune diseases [35, 36]. Remarkably, our data demonstrate that IL1B levels were increased in patients with neutrophilic, but not eosinophilic, asthma. Moreover, IL1B administration to epithelial cells increased glycolysis while blocking its signal attenuated HDM-induced glycolysis and dampened cytokine release. This is in line with evidence of the role played by IL1B in chronic obstructive pulmonary disease (COPD), whereby IL1B activates macrophages in these patients to produce inflammatory cytokines, chemokines and MMPs [37, 38]. Ongoing studies are currently aiming to study the efficacy of blocking IL1B signaling in COPD patients [39]. Additionally, elevated serum IL1B levels were associated with more inflammation in severe allergic rhinitis patients [40]. Together, our results confirm a pivotal role played by IL1B and increased glycolysis in the pathogenesis of asthma and suggest the use of increased IL1B levels and markers of glycolysis such as lactate (discussed below) in sputum or serum samples as biomarkers in asthma, specifically the neutrophilic subtype.

\section{Elevated lactate as a biomarker in asthma}

Lactate, instead of being considered as a waste product, is now recognized as a fuel source. Some studies show that lactate can be used as a precursor for glycogenesis as well as gluconeogenesis, whereby lactate is transported through the blood to the liver where it gets converted into pyruvate that will be used for glucose synthesis [41]. This suggest that lactate can be used as an immediate energy source or as a contributor to the energy reserve [42]. An interesting recent study utilizing ${ }^{13} \mathrm{C}$-lactate metabolomics revealed that in fact glucose feeds the TCA cycle through the circulating lactate [43]. Although being produced by most tissues, muscles generate the highest amount of lactate in the body [44]. Under resting conditions, the amount of lactate production and consumption are approximately equal, resulting in a very low blood lactate level (0.5- $1 \mathrm{mmol} / \mathrm{l})$ [45]. High concentration of lactate, however, occurs in several conditions such as hypoxia, shock, and intense exercise [44]. In our studies, we show that sputum samples from asthmatics had higher levels of lactate which correlated negatively with lung function (\%FEV1) compared to healthy individuals. In addition, nasal cells isolated from asthmatics displayed higher expression of LDHA, the glycolytic enzyme responsible for the conversion of pyruvate to lactate (chapter 3). Moreover, altered energy metabolism with significant increases in lactate levels, were noted in an ovalbumin-induced asthma model and were associated with recruitment of inflammatory cells [46]. In accordance with our results, others have shown that 
higher pulmonary lactate levels were detected in response to acute lung injury and sepsis, which was linked to a more severe form of the disease [47, 48]. Lactate levels in sputum of patients with acute lower respiratory tract infection were correlated with neutrophil invasion in another study [49]. Interestingly, our data demonstrate higher levels of lactate in sputum samples from patients with neutrophilic asthma compared to healthy individuals. These higher levels of lactate positively correlate with IL1B, but negatively correlate with lung function as mentioned above (chapter 3). Furthermore, activation of the glycolytic enzyme PKM2, using TEPP46, attenuated the increases in IL1B- induced lactate secretion in lung epithelial cells (chapter 4), pointing at a potential role played by epithelial PKM2 in lactate regulation. Altogether, our data propose the use of elevated lactate in sputum, along with increases in IL1B, as biomarkers for asthma, specifically the neutrophilic subtype. These easy to measure non-invasive biomarkers will help physicians position their patients in their right subcategories in order to choose their appropriate therapeutic regimen. Additionally, a thorough investigation of the mechanism behind and the role played by elevated lactate in asthma pathogenesis is necessary to better understand its contribution to the disease process.

\section{PKM2: lessons from cancer and implications in asthma}

Further exploration of the mechanism by which glycolytic alterations contribute to the allergic airways disease yielded interesting findings for pyruvate kinase PKM2. PK catalyzes the conversion of PEP to pyruvate, one of the most important and irreversible steps in glycolysis. Interestingly, our analysis shows increases in PKM2 levels both in nasal cells derived from asthmatics and in mice with HDM-induced allergic airways disease (chapter 3, 4). In addition, we demonstrate that activation of PKM2 by TEPP46 attenuates subepithelial collagen, mucus metaplasia, airway remodeling and allergen induced inflammation in mice with HDM-induced allergic airways disease (chapter 3), confirming PKM2's role in asthma pathogenesis.

PKM2 is the major isoform of PK that is expressed in cancer cells. Blood, serum and stool samples collected from cancer patients were reported with elevated levels of PKM2 [50-52]. Additionally, increases in PKM2 levels have been correlated with tumor size and stage [51]. PKM2 exists in a low active dimer or a high active tetramer isoform, as discussed earlier, with each isoform dictating different biological actions [53]. Interestingly, the switch between the two isoforms has been shown to enable tumor cells to survive in an environment with varying oxygen and nutrient supply [51]. Aside from its glycolytic function, many studies reported that PKM2 plays several non-glycolytic roles. For instance, PKM2 was demonstrated to directly interact with hypoxia-inducible factor 1 (HIF-1) subunit, promoting transactivation of its target genes. 
Interestingly, HIF-1 can also activate transcription of PKM2, thus creating a feedback loop that reprograms metabolism in cancer cells [54]. Nuclear translocation of PKM2 has also been described to play a role in cell proliferation in response to different events, such as IL-3 stimulation and EGFR activation [55, 56]. In the nucleus, PKM2 acts as a protein kinase that phosphorylates STAT3 and enhances transcription of several cancer related genes [57]. Interestingly, in our studies, PKM2 was indeed able to phosphorylate STAT3 and enhance the inflammatory events in lung epithelial cells (chapter 4). PKM2 can be regulated at multiple levels. It is worthy to note here, that PKM2 oxidation at cysteine 358 was shown to inhibit its activity and promote diversion to PPP, producing NADPH necessary for redox balance, pointing at a potential role of PKM2 in redox regulation. Expression of an oxidation resistant PKM2 mutant interestingly increased oxidative stress and inhibited tumor growth [58]. This suggest that PKM2 activators can be beneficial in cancer therapeutics, especially when combined with radiation or chemotherapy. Indeed, the small molecule activators of PKM2, TEPP46, showed drastic effects on lung and breast tumor cell viability when combined with the glucose analog 2DG in another study [59]. Paradoxically to activating PKM2, inhibiting the enzyme's activity, using small interfering RNA (siRNA), also increased apoptosis and inhibited tumor growth in mouse xenograft model [60]. These conflicting results warrant more studies to further elaborate the benefits of activating or inhibiting PKM2 in disease settings.

Although PKM2 has been extensively studied in cancer, its relevance to other diseases is still under explored. In allergic airways disease, we have shown that glycolytic reprogramming and PKM2, are critical for the disease pathogenesis. In addition, activating PKM2 using TEPP46 attenuated inflammatory cytokines, notably TSLP and GM-CSF (chapter 3, 4). Many lessons can be learned from the cancer field, where PKM2 was most studied, and tested for their relevance in asthma and allergic airways disease. For example, a clinical trial using the PKM2 activator, TP15454, was recently announced to start aiming to test the effect of activating PKM2 on the progression of patients with metastatic and progressive tumors. Given that glycolytic reprogramming is critical to asthma pathogenesis, preclinical studies should aim at examining the efficacy and safety of targeting PKM2 as potential treatment for asthmatics who display elevated glycolysis in their lungs. Murine asthma models can be a great tool to further depict these goals. For instance, and in addition to HDM- induced allergic airways disease, ovalbumin (OVA) can be used to model eosinophilic asthma, while lipopolysaccharide (LPS), in combination with OVA can be used to model neutrophilic subtype [61]. These animal studies are necessary to test the feasibility of targeting PKM2 in the different asthma subtypes. PKM2 is a key player in the glycolysis pathway and is known to play a major role in immune response, in addition to the 
paradoxical effects of activating and inhibiting it. Therefore, it is critical to carefully monitor all the possible side effects induced by manipulating (whether activating or inhibiting) PKM2 before proposing its use in asthma clinical trials. Great attention should also be given to the different characteristics that may impact the results and findings of these studies such as age, sex and weight.

\section{Metabolic reprogramming and redox homeostasis: crosstalk in lung epithelial cells and asthma pathogenesis}

As described earlier, the link between metabolic reprogramming and redox balance has been reported in cancer, whereby cancer cells enhance their metabolic capacity to keep up with the high nutrient demand. In addition, upregulation of multiple enzymatic and non-enzymatic antioxidant mechanisms, that function to scavenge the elevated ROS levels, has also been described as a hallmark of cancer, with elevated levels of glutathione being one of the key players in this regulation [62]. This link between metabolic reprogramming and redox homeostasis, notably Sglutathionylation, is however understudied. In chapter 5 and 6 , we focused our investigations on redox chemistry and metabolic reprogramming and whether one is regulating the other in lung epithelial cells. We employed a multi-omics approach consisting of glutathionylated proteome, metabolome and DNA array and ran unbiased analysis to study overlapping pathways in the three platforms. Our metabolomics analysis revealed that glycolytic reprogramming indeed occurs in epithelial cells stimulated with IL1B, further confirming our previous findings of IL1B's role in inducing glycolysis (chapter 3, 4, 5). In addition, multiple glycolytic intermediates were upregulated in cells lacking Glrx, the enzyme responsible for deglutathionylation, therefore linking metabolic reprogramming to redox biochemistry. Interestingly, in response to IL1B, and as expected in Glrx ${ }^{-/}$cells, multiple proteins were shown to be S-glutathionylated. Kyoto Encyclopedia of Genes and Genomes (KEGG) pathway analysis clustered these proteins in multiple pathways including carbon metabolism, biosynthesis of amino acids, and metabolic pathways. Importantly, the glycolysis pathway contained many S-glutathionylated intermediates in response to IL1B stimulation and in absence of Glrx, further proposing that S-glutathionylation might be a regulator of metabolic reprogramming in epithelial cells. This is also suggestive of a vicious cycle type of regulation whereby glycolytic reprogramming affects redox balance and vice versa.

Interestingly, PKM2 was one of the identified targets in our unbiased glutathionylated proteome screening, which suggests that PKM2-S-glutathionylation may contribute to the enhanced metabolic reprogramming induced by IL1B or absence of Glrx. In fact, recent work by 
our group demonstrates that S-glutathionylation of PKM2 was indeed associated with metabolic reprogramming and accompanied by enhanced inflammatory cytokine production in a mouse model of obese allergic airways disease (In review for publication). Moreover, our work in chapter 5 , shows the enhanced release of pro-inflammatory asthma-relevant cytokines, notably TSLP and to lesser extent GM-CSF, in Glrx $x^{-/}$epithelial cells (chapter 5). Additionally, s-glutathionylation can also modulate inflammation via the direct targeting of key players in the inflammatory signaling pathways, such as NF-kB. The transcription factor NF-kB that plays a pivotal role in inflammation, has been shown to be modulated by S-glutathionylation through different mechanisms. In particular, the p65 subunit of NF-KB was reported to be S-glutathionylated under certain conditions [63]. In addition, another study demonstrated that IKK, the protein responsible for IKB phosphorylation and NFKB translocation to the nucleus, is also a target for S-glutathionylation at cysteine 179 leading to its inactivation [64]. Interestingly, a recent publication demonstrates that IL1B itself can be S-glutathionylated at cysteine 188 which protects the cytokine from irreversible oxidation and positively regulates its activity [65]. All these studies, along with ours, suggest a major role played by S-glutathionylation in regulating metabolic reprogramming as well as inflammation. Therefore, targeting S-glutathionylation might provide a useful therapeutic approach in pathologies with metabolic perturbations such as asthma. S-glutathionylation, however, is very complex and cannot be looked at as one single entity, instead S-glutathionylation of each target protein may have different biological effects, even if these proteins are in a shared common pathway. Therefore, identification of key S-glutathionylated targets in the inflammatory (and other) pathways will provide more viable therapeutic targets to overcome side effects of interfering with overall S-glutathionylation.

\section{System $x_{c}:$ : a new potential target to combat pro-inflammatory signaling}

Various mechanisms have been described by which cancer cells increase their GSH content to combat oxidative stress [66]. Amongst these, upregulation of system $\mathrm{x}_{\mathrm{c}}{ }^{-}$has grabbed scientists' attention in the last decade for multiple reasons. The antiporter system $x_{c}^{-}$is upregulated in multiple cancer subtypes which correlates with more aggressive tumor and resistance to therapy [67-69]. Beside its crucial role in importing cystine necessary for GSH synthesis, system $x_{c}^{-}$'s export of glutamate is of equal significance. The transporter indeed is considered an important non-vesicular extrasynaptic source of glutamate needed for the neurotransmitter homeostasis [70]. This although very important, was not the focus of our studies and will not be discussed in this thesis. In our experiments, we demonstrate increases in SLC7A11, the active subunit of system $\mathrm{x}_{c}^{-}$, levels in response to IL1B and in cells lacking GIrx, 
two scenarios in which S-glutathionylation is increased. These increases in SLC7A11 levels, as expected, translated to higher amounts of GSH (chapter 6). Blood as well as lung samples from asthmatics typically have higher levels of GSH that relate to the extent of airway inflammation and are therefore regarded as an adaptive response to the high oxidative environment [71]. Interestingly, we demonstrate that inhibiting GSH with a system $\mathrm{x}_{c}{ }^{-}$inhibitor (or a GCL inhibitor) has favorable impact on lowering TSLP levels in epithelial cells (chapter 6). These observations point at an important role played by $\mathrm{GSH}$ and system $\mathrm{x}_{\mathrm{c}}^{-}$in regulating the pro-inflammatory signaling, and further confirm that the IL1B-induced increases in GSH is more than just an adaptive response to the increased inflammation and oxidative environment. In fact, a recent study showed the involvement of system $\mathrm{x}_{c}^{-}$in peripheral and central inflammation in an in vivo model of LPS-induced sickness and depression-like behavior in mice [72]. Moreover, increased system $\mathrm{x}_{c}^{-}$expression has been associated with inflammation and lower antimicrobial function in the development of tuberculosis (TB). Interestingly, inhibiting $x_{c}^{-}$or its genetic depletion reduced bacterial load and TB pathology in the lungs [73]. Collectively, these data point at a direct therapeutic impact of targeting $x_{c}{ }^{-}$on several inflammatory diseases, including allergic airway disease. However, careful monitoring of the oxidative stress levels and potential oxidative damage is necessary in trials involving system $x_{c}^{-}$inhibition. Importantly, more studies should take advantage of the orally administered FDA approved drug sulfasalazine ( $\mathrm{x}_{c}^{-}$inhibitor), that has been used for years in treatment of inflammatory disorders such as Crohn's disease, ulcerative colitis, and inflammatory bowel disease [67], and examine its effects on other inflammatory diseases like asthma.

\section{GSH promotes its own synthesis in an S-glutathionylation-dependent mechanism}

Our results demonstrate that elevated levels of SLC7A11 and GSH are linked to higher levels of S-glutathionylation (in IL1B and $G / x^{-/}$cells), specifically to OTUB1 S-glutathionylation (OTUB1-SSG). Moreover, administering recombinant GLRX protein, lowered overall protein Sglutathionylation, OTUB1-SSG, SLC7A11 as well as GSH levels. In addition to its regulation by phosphorylation [74], our data herein describe a new post-translational mechanism by which system $\mathrm{x}_{\mathrm{c}}^{-}$can be regulated.

The antioxidant GSH is extensively regulated to maintain the fine redox homeostasis. GSH has been described to negatively regulate its own synthesis by inhibiting GCL [75]. Our findings here however highlight a positive feedforward regulation by which GSH increases its own synthesis through S-glutathionylation. In addition to our findings, S-glutathionylation of cystathionine $\beta$-synthase (CBS), a key enzyme in the trans-sulfuration pathway, was shown to 
enhance the enzyme activity, leading to increases in cysteine synthesis, the rate limiting amino acid in GSH biosynthesis [76]. KEAP1 S-glutathionylation is also another mechanism that promotes GSH synthesis through SLC7A11 upregulation [77]. Together, these results point at the intricate regulation of $\mathrm{GSH}$, and a crucial role played by S-glutathionylation in fine-tuning it. Any dysregulation of the GSH delicate balance can be an inducer and/or contributor to different diseases as discussed earlier. From the classical oxidative damage perspective, the therapeutic aim has been focused on increasing the antioxidant GSH levels in general, however these approaches failed to provide therapeutic benefits. Therefore, detailed understanding of the exact mechanism(s) underlying changes in GSH homeostasis and its contribution to certain disease is necessary before interfering with its signaling. This will help in designing better tailored approaches that will lower many of the off-target effects. For instance, interfering with OTUB1SSG in settings such as cancers, where increases of GSH were due to SLC7A11 overexpression, will provide more therapeutic benefits, with less side effects, than completely blocking GSH synthesis.

Therapeutic roles of GLRX recombinant protein have recently emerged in multiple diseases, including cancer, cardiovascular diseases, and lung fibrosis [78-80]. Our data suggest that GLRX may also have therapeutic benefits in diseases with elevated GSH levels, including asthma. More studies however are needed to further confirm and validate these findings.

\section{Targeting TSLP in therapy for severe asthma}

TSLP is a member of the alarmin family of cytokines that also includes IL-33 and IL-25. TSLP is primarily expressed by airway epithelial cells and is released in response to allergens, viruses, and other insults [81]. TSLP expression was found to be increased in airways of patients with asthma, and it has been shown to play a crucial role in the disease pathophysiology, correlating with its severity and lung function impairment [82]. Following its release from epithelium, the cytokine activates dendritic cells to induce Th2 allergic responses [83, 84]. In neutrophilic asthma, TSLP activates dendritic cells and induces polarization of $T$ cells towards a Th17 phenotype that is associated with severe asthma $[85,86]$. TSLP was also shown to play roles in airway remodeling [87], in stimulating collagen production from fibroblasts [88] and in the crosstalk between mast and airway smooth muscle cells [89]. Interestingly, a recent clinical trial shows that patients with severe uncontrolled asthma receiving the monoclonal TSLP blocker, Tezepelumab, had fewer asthma exacerbations, better lung function and asthma control compared to patients receiving the placebo drug [90]. In this thesis, we demonstrate that in response to IL1B administration to epithelial cells, TSLP released in cell culture supernatant was 
significantly increased (chapter 3-6). These increases were attenuated when glycolysis or glutathione were inhibited or with PKM2 activation. Additionally, we demonstrate higher levels of TSLP in Glrx ${ }^{-/}$cells stimulated with IL1B compared to the WT cells, further suggesting a role played by PSSG in TSLP regulation. More studies however are needed to elaborate if increases in overall S-glutathionylation or of a specific target is eliciting these effects. Overall, our data, along with others, highlight the importance of TSLP signaling in asthma pathogenesis and point at the importance of understanding its mechanistic regulation. Careful examination of sputum and blood levels of IL1B, GSH and PKM2 in subjects enrolled in the Tezepelumab clinical trial will provide invaluable information about these players' involvement in TSLP signaling in severe asthma, and therefore is strongly recommended.

\section{Implications beyond asthma}

Finally, we would like to highlight that our findings may have translational values in diseases other than asthma. Metabolic reprogramming has been linked to multiple diseases and syndromes including diabetes, cancer, and obesity [91-93]. In addition, elevations in IL1B levels were found to be associated with numerous inflammatory conditions such as Alzheimer's, Parkinson's, stroke, atherosclerosis, and diabetes [94-98]. Results in this thesis suggest that neutralizing IL1B signaling dampens pro-inflammatory cytokines and therefore may provide desirable effects in other diseases with elevated IL1B as an underlying mechanism. Interestingly, anakinra, which is an IL1 receptor blocker, is an approved drug that is used in treatment of rheumatoid arthritis, gout, and idiopathic pericarditis. More clinical studies should test the effects of anakinra and IL1B inhibition in other diseases with aberrant IL1B signaling. Importantly, elevated levels of circulating and plasma IL1B were detected in COVID-19 patients accompanied by neutrophilic flux and severe symptoms [99]. Lactate and PKM2 expressions were also higher in COVID-19 patients, in addition to elevated levels of lactate dehydrogenase (the enzyme responsible for generating lactate) $[99,100]$.

All the aforementioned observations highlight the relevance of our work in several inflammatory diseases other than asthma.

Cancer is characterized by both metabolic reprogramming and changes in redox homeostasis. Our results show that inhibiting glycolysis and/or glutathione have desirable effects on decreasing pro-inflammatory cytokines in asthma. In addition, we propose the SLC7A11/ OTUB1-SSG as a mechanism by which GSH is regulated. In fact, many cancer subtypes have elevated levels of SLC7A11 and/ or OTUB-1 [101, 102]. The higher expression of these two proteins is linked to aggressive cancers with metastasis and resistance to therapy [103, 104]. We 
speculate that the increases in GSH might be a contributor to these events. Therefore, our new identified mechanism may provide an explanation and help in better drug design, which will have a great impact on the cancer field. Furthermore, our data highlight a new mechanism by which system $x_{c}{ }^{-}$itself is regulated (through OTUB1-SSG). In addition to its involvement in cancer, system $x_{c}^{-}$plays a major role in the physiology and pathophysiology of several neurologic and inflammatory diseases [105]. In fact, sulfasalazine, a system $x_{c}{ }^{-}$inhibitor, is an FDA approved drug used in patients with rheumatoid arthritis, Crohn's disease, ulcerative colitis and others. Our mechanistic findings may provide a valuable tool in designing more therapeutics to target system $\mathrm{X}_{\mathrm{c}}^{-}$.

Concerns usually arise when scientists propose inhibition of a common pathway (such as glycolysis or glutathione), due to its implications and involvement in many processes. Taking glycolysis pathway for instance, although its inhibition may provide beneficial effects against several diseases including cancer, other organs and tissues will be drastically affected as well. Glucose is the main energy source for multiple organs including the brain, and inhibiting glycolysis will be potentially toxic and damaging to these organs. One way of overcoming these off-target effects is to design inhibitors that do not cross the blood brain barrier for example and therefore protect the brain, however other organs will still be affected [106]. Another solution is to use combination therapy where physicians can benefit from titrating down the dose of the inhibitor, to correct the disease without affecting normal cells. This however, in many cases, is unrealistic and very difficult to be accomplished. Scientists faced same obstacles when they tested GSH inhibition in cancer where they found that total GSH depletion was not effective as a single treatment, but provided promising results when combined with other cancer chemotherapeutics [107]. In addition, studies with $\mathrm{N}$-acetylcysteine (NAC), a widely used antioxidant, provided mixed results probably owing to the nature of cancer and whether it relies on ROS for survival and metastasis [108]. Therefore, identifying specific targets and/or regulators of complex pathways such as glutathione and glycolysis has the potential to help towards the development of improved therapeutics.

\section{Limitations and unexplored areas}

As explained earlier, asthma heterogeneity, the presence of big overlap between its subtypes as well as the different response to therapy urge the identification of new biomarkers and an in-depth understanding of molecular mechanisms governing the disease pathophysiology. Our data herein suggest that increases in glycolysis and IL1B are hallmarks of neutrophilic asthma. We additionally propose elevated lactate, as an indicator for the enhanced glycolysis, as 
a promising biomarker for neutrophilic asthma patients, in addition to increases in IL1B. Our multiomics analysis indeed showed that not only lactate but also several other metabolites are increased in response to IL1B. Further tests are needed to determine if other glycolytic metabolites can also be used in the characterization of asthmatics. Moreover, our conclusions were derived after performing analysis in vitro and in vivo (primary epithelial cells, mice and human samples). However, more clinical samples derived from asthmatic patients are needed to confirm our results. Attention should be given to different patients' characteristics including sex, age, weight, and medications before drawing solid conclusions.

In addition to glycolytic reprogramming and enhanced IL1B signaling and their role in asthma pathogenesis, we also demonstrate that neutralizing IL1 signaling attenuates lactate, HKII and LDHA levels, confirming the role of IL1 in glycolytic reprogramming. The IL1 receptor antagonist anakinra has far more off-label uses beside being a drug used for rheumatoid arthritis [109]. Giving its promising results in patients with other inflammatory diseases, it will be very interesting to test the effect of anakinra in patients with neutrophilic asthma.

To study the cross talk between glycolytic reprogramming and redox homeostasis, we utilized a multi-omics approach, which we believe was invaluable and provided substantial amount of data. Beside glycolysis and TCA cycle (that we focused on in chapter 5), there are multiple other pathways that overlapped between the three omics platforms and warrant investigations. Among these, the glutathione synthesis and degradation pathway represents one of the greatest interest due to its direct link with redox homeostasis. Metabolic pathways as well as biosynthesis of amino acids are two other examples of pathways that are of high relevance and worth attention. We therefore think that our study in chapter 5 provides valuable information that can be used to direct future science.

As a rule of thumb, testing a hypothesis starts with performing analysis in cells (in vitro), then moves to in vivo animal models before reaching clinical stages. In this thesis, we unravel a new mechanism by which system $x_{c}^{-}$, and subsequently $\mathrm{GSH}$, are regulated. In addition, we demonstrate beneficial effects of inhibiting system $\mathrm{x}_{\mathrm{c}}{ }^{-}$and/ or GSH in epithelial cells. Further in vivo studies are therefore needed to validate our findings. In addition to HDM-induced allergic airways disease as a model to corroborate our results, it will be interesting to expand these in vivo studies beyond asthma to include other inflammatory conditions.

Our findings in chapter 6 might also have an impact on the cancer field, due to its relevance to some cancer subtypes with high SLC7A11 expression. Xenograft studies aiming to test S-glutathionylation/ SLC7A11/ GSH paradigm may indeed provide important value in cancer, especially given that SLC7A11-high cancers are severe, aggressive, and resistant to therapy. 


\section{Overall Conclusion}

Deciphering the mechanism(s) underlying a complex and heterogeneous disease like asthma, is of great importance in order to develop better therapeutics. In addition, identifying biomarkers for the several asthma phenotypes will have a great impact on the disease management. Results in this thesis highlight glycolytic reprogramming and redox perturbations crosstalk, and their relevance to asthma pathogenesis. We specifically show that IL1B signaling contributes to asthma pathogenesis by driving glycolytic reprogramming, increasing inflammation, and inducing airway hyperresponsiveness and remodeling. In addition, we propose IL1B and lactate as potential non-invasive biomarkers in asthma patients, notably the ones with neutrophilic phenotype. Additionally, we showed beneficial effects of activating the glycolytic enzyme PKM2 in the attenuation of airway remodeling and inflammation. These promising findings represent PKM2 as a potential therapeutic target for asthmatics with elevated IL1B and glycolysis (such as neutrophilic asthmatics).

Interestingly, results here demonstrate that S-glutathionylation chemistry has a great impact on multiple metabolic pathways in epithelial cells, notably on glycolysis. In fact, we demonstrate that not only glycolysis contributes to redox homeostasis through the PPP, but also that redox chemistry regulates glycolysis at multiple levels. In addition, we explain a sophisticated mechanism by which GSH regulates its own synthesis through an S-glutathionylation and system $\mathrm{x}_{\mathrm{c}}{ }^{-}$- dependent axis, which interestingly modulates the pro-inflammatory signaling. The overall outcome of this thesis provides new insights on how metabolic changes and redox imbalance promote inflammation in allergic lung disease, notably asthma. 


\section{$\underline{\text { References: }}$}

1. Warburg, O., On the origin of cancer cells. Science, 1956. 123(3191): p. 309-14.

2. Lunt, S.Y. and M.G. Vander Heiden, Aerobic glycolysis: meeting the metabolic requirements of cell proliferation. Annu Rev Cell Dev Biol, 2011. 27: p. 441-64.

3. Xie, N., et al., Glycolytic Reprogramming in Myofibroblast Differentiation and Lung Fibrosis. Am J Respir Crit Care Med, 2015. 192(12): p. 1462-74.

4. Marelli-Berg, F.M., H. Fu, and C. Mauro, Molecular mechanisms of metabolic reprogramming in proliferating cells: implications for T-cell-mediated immunity. Immunology, 2012. 136(4): p. 3639.

5. Goodwin, J., et al., Targeting Hypoxia-Inducible Factor-1 $\alpha /$ Pyruvate Dehydrogenase Kinase 1 Axis by Dichloroacetate Suppresses Bleomycin-induced Pulmonary Fibrosis. Am J Respir Cell Mol Biol, 2018. 58(2): p. 216-231.

6. Agathocleous, M. and W.A. Harris, Metabolism in physiological cell proliferation and differentiation. Trends Cell Biol, 2013. 23(10): p. 484-92.

7. Ho, T.T., et al., Autophagy maintains the metabolism and function of young and old stem cells. Nature, 2017. 543(7644): p. 205-210.

8. $\quad$ Andersen, J.L. and S. Kornbluth, The tangled circuitry of metabolism and apoptosis. Mol Cell, 2013. 49(3): p. 399-410.

9. Eming, S.A., T.A. Wynn, and P. Martin, Inflammation and metabolism in tissue repair and regeneration. Science, 2017. 356(6342): p. 1026-1030.

10. Kim, J., J. Kim, and J.S. Bae, ROS homeostasis and metabolism: a critical liaison for cancer therapy. Exp Mol Med, 2016. 48(11): p. e269.

11. Sbodio, J.I., S.H. Snyder, and B.D. Paul, Redox Mechanisms in Neurodegeneration: From Disease Outcomes to Therapeutic Opportunities. Antioxid Redox Signal, 2019. 30(11): p. 1450-1499.

12. Choi, M.H., et al., Oxidative Stress-Mediated Skeletal Muscle Degeneration: Molecules, Mechanisms, and Therapies. Oxid Med Cell Longev, 2016. 2016: p. 6842568.

13. Griffiths, H.R., D. Gao, and C. Pararasa, Redox regulation in metabolic programming and inflammation. Redox Biol, 2017. 12: p. 50-57.

14. Van den Bossche, J., L.A. O'Neill, and D. Menon, Macrophage Immunometabolism: Where Are We (Going)? Trends Immunol, 2017. 38(6): p. 395-406.

15. Dweik, R.A., et al., NO chemical events in the human airway during the immediate and late antigen-induced asthmatic response. Proc Natl Acad Sci U S A, 2001. 98(5): p. 2622-7.

16. Gaston, B., et al., The biology of nitrogen oxides in the airways. Am J Respir Crit Care Med, 1994. 149(2 Pt 1): p. 538-51.

17. MacPherson, J.C., et al., Eosinophils are a major source of nitric oxide-derived oxidants in severe asthma: characterization of pathways available to eosinophils for generating reactive nitrogen species. J Immunol, 2001. 166(9): p. 5763-72.

18. McDowell, P.J. and L.G. Heaney, Different endotypes and phenotypes drive the heterogeneity in severe asthma. Allergy, 2020. 75(2): p. 302-310.

19. Bell, S.M., et al., Peripheral Glycolysis in Neurodegenerative Diseases. Int J Mol Sci, 2020. 21(23).

20. Zhang, Y., et al., Glycolysis-Related Genes Serve as Potential Prognostic Biomarkers in Clear Cell Renal Cell Carcinoma. Oxid Med Cell Longev, 2021. 2021: p. 6699808.

21. Ostroukhova, M., et al., The role of low-level lactate production in airway inflammation in asthma. Am J Physiol Lung Cell Mol Physiol, 2012. 302(3): p. L300-7.

22. $\mathrm{Xu}, \mathrm{Y} . \mathrm{D}$. , et al., The early asthmatic response is associated with glycolysis, calcium binding and mitochondria activity as revealed by proteomic analysis in rats. Respir Res, 2010. 11(1): p. 107. 
23. Kim, Y.H., T. Nakayama, and J. Nayak, Glycolysis and the Hexosamine Biosynthetic Pathway as Novel Targets for Upper and Lower Airway Inflammation. Allergy Asthma Immunol Res, 2018. 10(1): p. 6-11.

24. Xu, W., et al., Platelets from Asthmatic Individuals Show Less Reliance on Glycolysis. PLoS One, 2015. 10(7): p. e0132007.

25. Larsen, C.M., et al., Interleukin-1-receptor antagonist in type 2 diabetes mellitus. N Engl J Med, 2007. 356(15): p. 1517-26.

26. Rébé, C. and F. Ghiringhelli, Interleukin-16 and Cancer. Cancers (Basel), 2020. 12(7).

27. Kaneko, N., et al., The role of interleukin-1 in general pathology. Inflamm Regen, 2019. 39: p. 12.

28. Dinarello, C.A., Interleukin-1, interleukin-1 receptors and interleukin-1 receptor antagonist. Int Rev Immunol, 1998. 16(5-6): p. 457-99.

29. Sousa, A.R., et al., Expression of interleukin-1 beta (IL-1beta) and interleukin-1 receptor antagonist (IL-1ra) on asthmatic bronchial epithelium. Am J Respir Crit Care Med, 1996. 154(4 Pt 1): p. 10616.

30. Pujol, J.L., et al., Interleukin-1 release by alveolar macrophages in asthmatic patients and healthy subjects. Int Arch Allergy Appl Immunol, 1990. 91(2): p. 207-10.

31. Hakonarson, H., et al., Autocrine role of interleukin 1 beta in altered responsiveness of atopic asthmatic sensitized airway smooth muscle. J Clin Invest, 1997. 99(1): p. 117-24.

32. Ben-Sasson, S.Z., et al., IL-1 acts directly on CD4 T cells to enhance their antigen-driven expansion and differentiation. Proc Natl Acad Sci U S A, 2009. 106(17): p. 7119-24.

33. Mahmutovic Persson, I., et al., IL-16 mediates lung neutrophilia and IL-33 expression in a mouse model of viral-induced asthma exacerbation. Respir Res, 2018. 19(1): p. 16.

34. Fahy, J.V., Type 2 inflammation in asthma--present in most, absent in many. Nat Rev Immunol, 2015. 15(1): p. 57-65.

35. Park, M.J., et al., IL-1 Receptor Blockade Alleviates Graft-versus-Host Disease through Downregulation of an Interleukin-16-Dependent Glycolytic Pathway in Th17 Cells. Mediators Inflamm, 2015. 2015: p. 631384.

36. Newcomb, D.C. and R.S. Peebles, Jr., Th17-mediated inflammation in asthma. Curr Opin Immunol, 2013. 25(6): p. 755-60.

37. $\mathrm{Yi}, \mathrm{G} .$, et al., A large lung gene expression study identifying IL1B as a novel player in airway inflammation in COPD airway epithelial cells. Inflamm Res, 2018. 67(6): p. 539-551.

38. Lappalainen, U., et al., Interleukin-1beta causes pulmonary inflammation, emphysema, and airway remodeling in the adult murine lung. Am J Respir Cell Mol Biol, 2005. 32(4): p. 311-8.

39. Culpitt, S.V., et al., Impaired inhibition by dexamethasone of cytokine release by alveolar macrophages from patients with chronic obstructive pulmonary disease. Am J Respir Crit Care Med, 2003. 167(1): p. 24-31.

40. Han, M.W., et al., Serum IL-16 can be a biomarker in children with severe persistent allergic rhinitis. Allergy Asthma Clin Immunol, 2019. 15: p. 58.

41. Stevenson, R.W., et al., Lactate as substrate for glycogen resynthesis after exercise. J Appl Physiol (1985), 1987. 62(6): p. 2237-40.

42. Nalbandian, M. and M. Takeda, Lactate as a Signaling Molecule That Regulates Exercise-Induced Adaptations. Biology (Basel), 2016. 5(4).

43. Hui, S., et al., Glucose feeds the TCA cycle via circulating lactate. Nature, 2017. 551(7678): p. 115118.

44. Andersen, L.W., et al., Etiology and therapeutic approach to elevated lactate levels. Mayo Clin Proc, 2013. 88(10): p. 1127-40.

45. van Hall, G., Lactate kinetics in human tissues at rest and during exercise. Acta Physiol (Oxf), 2010. 199(4): p. 499-508. 
46. Ho, W.E., et al., Metabolomics reveals altered metabolic pathways in experimental asthma. Am J Respir Cell Mol Biol, 2013. 48(2): p. 204-11.

47. De Backer, D., et al., Lactate production by the lungs in acute lung injury. Am J Respir Crit Care Med, 1997. 156(4 Pt 1): p. 1099-104.

48. Brown, S.D., C. Clark, and G. Gutierrez, Pulmonary lactate release in patients with sepsis and the adult respiratory distress syndrome. J Crit Care, 1996. 11(1): p. 2-8.

49. Fredman, G., et al., The inflamed sputum in lower respiratory tract infection: I-lactate levels are correlated to neutrophil accumulation. Apmis, 2019. 127(2): p. 72-79.

50. Christofk, H.R., et al., The M2 splice isoform of pyruvate kinase is important for cancer metabolism and tumour growth. Nature, 2008. 452(7184): p. 230-3.

51. Mazurek, S., et al., Pyruvate kinase type M2 and its role in tumor growth and spreading. Semin Cancer Biol, 2005. 15(4): p. 300-8.

52. Lüftner, D., et al., Tumor type M2 pyruvate kinase expression in advanced breast cancer. Anticancer Res, 2000. 20(6d): p. 5077-82.

53. Mazurek, S., Pyruvate kinase type M2: a key regulator of the metabolic budget system in tumor cells. Int J Biochem Cell Biol, 2011. 43(7): p. 969-80.

54. Luo, W., et al., Pyruvate kinase M2 is a PHD3-stimulated coactivator for hypoxia-inducible factor 1. Cell, 2011. 145(5): p. 732-44.

55. Hoshino, A., J.A. Hirst, and H. Fujii, Regulation of cell proliferation by interleukin-3-induced nuclear translocation of pyruvate kinase. J Biol Chem, 2007. 282(24): p. 17706-11.

56. Yang, W., et al., Nuclear PKM2 regulates 8 -catenin transactivation upon EGFR activation. Nature, 2011. 480(7375): p. 118-22.

57. Gao, X., et al., Pyruvate kinase $\mathrm{M} 2$ regulates gene transcription by acting as a protein kinase. Mol Cell, 2012. 45(5): p. 598-609.

58. Anastasiou, D., et al., Inhibition of pyruvate kinase $\mathrm{M} 2$ by reactive oxygen species contributes to cellular antioxidant responses. Science, 2011. 334(6060): p. 1278-83.

59. Tee, S.S., et al., PKM2 activation sensitizes cancer cells to growth inhibition by 2-deoxy-D-glucose. Oncotarget, 2017. 8(53): p. 90959-90968.

60. Goldberg, M.S. and P.A. Sharp, Pyruvate kinase M2-specific siRNA induces apoptosis and tumor regression. J Exp Med, 2012. 209(2): p. 217-24.

61. Yu, Q.L. and Z. Chen, Establishment of different experimental asthma models in mice. Exp Ther Med, 2018. 15(3): p. 2492-2498.

62. Traverso, N., et al., Role of glutathione in cancer progression and chemoresistance. Oxid Med Cell Longev, 2013. 2013: p. 972913.

63. Qanungo, S., et al., Glutathione supplementation potentiates hypoxic apoptosis by Sglutathionylation of p65-NFkappaB. J Biol Chem, 2007. 282(25): p. 18427-18436.

64. Reynaert, N.L., et al., Dynamic redox control of NF-kappaB through glutaredoxin-regulated Sglutathionylation of inhibitory kappaB kinase beta. Proc Natl Acad Sci U S A, 2006. 103(35): p. 13086-91.

65. Zhang, X., et al., Positive Regulation of Interleukin-16 Bioactivity by Physiological ROS-Mediated Cysteine S-Glutathionylation. Cell Rep, 2017. 20(1): p. 224-235.

66. Kennedy, L., et al., Role of Glutathione in Cancer: From Mechanisms to Therapies. Biomolecules, 2020. 10(10).

67. Ji, X., et al., XCT (SLC7A11)-mediated metabolic reprogramming promotes non-small cell lung cancer progression. Oncogene, 2018. 37(36): p. 5007-5019.

68. Guo, W., et al., Disruption of xCT inhibits cell growth via the ROS/autophagy pathway in hepatocellular carcinoma. Cancer Lett, 2011. 312(1): p. 55-61. 
69. Yang, Y. and D. Yee, IGF-I regulates redox status in breast cancer cells by activating the amino acid transport molecule XC. Cancer Res, 2014. 74(8): p. 2295-305.

70. Mohan, A., et al., Molecular diffusion model of neurotransmitter homeostasis around synapses supporting gradients. Neural Comput, 2011. 23(4): p. 984-1014.

71. Reynaert, N.L., Glutathione biochemistry in asthma. Biochim Biophys Acta, 2011. 1810(11): p. 1045-51.

72. Albertini, G., et al., Genetic deletion of $x C T$ attenuates peripheral and central inflammation and mitigates LPS-induced sickness and depressive-like behavior in mice. Glia, 2018. 66(9): p. 18451861.

73. Cai, Y., et al., xCT increases tuberculosis susceptibility by regulating antimicrobial function and inflammation. Oncotarget, 2016. 7(21): p. 31001-13.

74. Gu, Y., et al., mTORC2 Regulates Amino Acid Metabolism in Cancer by Phosphorylation of the Cystine-Glutamate Antiporter xCT. Mol Cell, 2017. 67(1): p. 128-138.e7.

75. Hicks, L.M., et al., Thiol-based regulation of redox-active glutamate-cysteine ligase from Arabidopsis thaliana. Plant Cell, 2007. 19(8): p. 2653-61.

76. Niu, W.N., et al., S-glutathionylation enhances human cystathionine B-synthase activity under oxidative stress conditions. Antioxid Redox Signal, 2015. 22(5): p. 350-61.

77. Carvalho, A.N., et al., S-Glutathionylation of Keap1: a new role for glutathione S-transferase pi in neuronal protection. FEBS Lett, 2016. 590(10): p. 1455-66.

78. Chang, Y., et al., Redox Regulator GLRX Is Associated With Tumor Immunity in Glioma. Front Immunol, 2020. 11: p. 580934.

79. Burns, M., et al., Role of Glutaredoxin-1 and Glutathionylation in Cardiovascular Diseases. Int J Mol Sci, 2020. 21(18).

80. Anathy, V., et al., Reducing protein oxidation reverses lung fibrosis. Nat Med, 2018. 24(8): p. 11281135.

81. Roan, F., K. Obata-Ninomiya, and S.F. Ziegler, Epithelial cell-derived cytokines: more than just signaling the alarm. J Clin Invest, 2019. 129(4): p. 1441-1451.

82. Mitchell, P.D. and P.M. O'Byrne, Epithelial-Derived Cytokines in Asthma. Chest, 2017. 151(6): p. 1338-1344.

83. Gauvreau, G.M., et al., Thymic stromal lymphopoietin: its role and potential as a therapeutic target in asthma. Expert Opin Ther Targets, 2020. 24(8): p. 777-792.

84. Watson, B. and G.M. Gauvreau, Thymic stromal lymphopoietin: a central regulator of allergic asthma. Expert Opin Ther Targets, 2014. 18(7): p. 771-85.

85. Gao, H., S. Ying, and Y. Dai, Pathological Roles of Neutrophil-Mediated Inflammation in Asthma and Its Potential for Therapy as a Target. J Immunol Res, 2017. 2017: p. 3743048.

86. Tanaka, J., et al., Human TSLP and TLR3 ligands promote differentiation of Th17 cells with a central memory phenotype under Th2-polarizing conditions. Clin Exp Allergy, 2009. 39(1): p. 89-100.

87. Redhu, N.S., et al., Thymic stromal lymphopoietin induces migration in human airway smooth muscle cells. Sci Rep, 2013. 3: p. 2301.

88. Cao, L., et al., TSLP promotes asthmatic airway remodeling via p38-STAT3 signaling pathway in human lung fibroblast. Exp Lung Res, 2018. 44(6): p. 288-301.

89. Kaur, D., et al., Mast cell-airway smooth muscle crosstalk: the role of thymic stromal lymphopoietin. Chest, 2012. 142(1): p. 76-85.

90. Menzies-Gow, A., et al., Tezepelumab in Adults and Adolescents with Severe, Uncontrolled Asthma. N Engl J Med, 2021. 384(19): p. 1800-1809.

91. Martin, S.D. and S.L. McGee, Metabolic reprogramming in type 2 diabetes and the development of breast cancer. J Endocrinol, 2018. 237(2): p. R35-r46. 
92. Faubert, B., A. Solmonson, and R.J. DeBerardinis, Metabolic reprogramming and cancer progression. Science, 2020. 368(6487).

93. Michelet, X., et al., Metabolic reprogramming of natural killer cells in obesity limits antitumor responses. Nat Immunol, 2018. 19(12): p. 1330-1340.

94. Shaftel, S.S., W.S. Griffin, and M.K. O'Banion, The role of interleukin-1 in neuroinflammation and Alzheimer disease: an evolving perspective. J Neuroinflammation, 2008. 5: p. 7.

95. Stojakovic, A., et al., Role of the IL-1 Pathway in Dopaminergic Neurodegeneration and Decreased Voluntary Movement. Mol Neurobiol, 2017. 54(6): p. 4486-4495.

96. Murray, K.N., A.R. Parry-Jones, and S.M. Allan, Interleukin-1 and acute brain injury. Front Cell Neurosci, 2015. 9: p. 18.

97. Libby, P., Interleukin-1 Beta as a Target for Atherosclerosis Therapy: Biological Basis of CANTOS and Beyond. J Am Coll Cardiol, 2017. 70(18): p. 2278-2289.

98. Dinarello, C.A., M.Y. Donath, and T. Mandrup-Poulsen, Role of IL-1beta in type 2 diabetes. Curr Opin Endocrinol Diabetes Obes, 2010. 17(4): p. 314-21.

99. McElvaney, O.J., et al., Characterization of the Inflammatory Response to Severe COVID-19 IIIness. Am J Respir Crit Care Med, 2020. 202(6): p. 812-821.

100. Szarpak, L., et al., Lactate dehydrogenase level as a COVID-19 severity marker. Am J Emerg Med, 2021. 45: p. 638-639.

101. Lim, J.K.M., et al., Cystine/glutamate antiporter xCT (SLC7A11) facilitates oncogenic RAS transformation by preserving intracellular redox balance. Proc Natl Acad Sci U S A, 2019. 116(19): p. 9433-9442.

102. Saldana, M., et al., Otubain 1: a non-canonical deubiquitinase with an emerging role in cancer. Endocr Relat Cancer, 2019. 26(1): p. R1-r14.

103. Bekeschus, S., et al., xCT (SLC7A11) expression confers intrinsic resistance to physical plasma treatment in tumor cells. Redox Biol, 2020. 30: p. 101423.

104. Zhou, Y., et al., OTUB1 promotes metastasis and serves as a marker of poor prognosis in colorectal cancer. Mol Cancer, 2014. 13: p. 258.

105. Lewerenz, J., et al., The cystine/glutamate antiporter system x(c)(-) in health and disease: from molecular mechanisms to novel therapeutic opportunities. Antioxid Redox Signal, 2013. 18(5): p. 522-55.

106. Pelicano, H., et al., Glycolysis inhibition for anticancer treatment. Oncogene, 2006. 25(34): p. 4633-46.

107. Desideri, E., F. Ciccarone, and M.R. Ciriolo, Targeting Glutathione Metabolism: Partner in Crime in Anticancer Therapy. Nutrients, 2019. 11(8).

108. Kwon, Y., Possible Beneficial Effects of N-Acetylcysteine for Treatment of Triple-Negative Breast Cancer. Antioxidants (Basel), 2021. 10(2).

109. Cavalli, G. and C.A. Dinarello, Anakinra Therapy for Non-cancer Inflammatory Diseases. Front Pharmacol, 2018. 9: p. 1157. 


\section{CHAPTER 8: SUMMARY}


The goal of this $\mathrm{PhD}$ thesis was to understand how changes in redox biochemistry affect cellular metabolism and whether this plays a role in the biology of lung epithelial cells and allergic lung diseases. We started our investigations by examining the importance of metabolic reprogramming in allergic airways disease, before linking these changes with the redox environment. Work herein highlights glycolytic reprogramming and redox perturbations as contributors in the pathophysiology of allergic airways disease (in mice) and in asthma. In addition, we describe a new complex mechanism by which glutathione, a major determinant of redox homeostasis, regulates its own synthesis with relevance to inflammatory allergic lung diseases, notably asthma.

After the introduction of key players investigated in this thesis along with the supportive background and the study questions (chapter 1 and 2), we first explored the importance of metabolic alterations in the development of allergic airway disease in mice. In chapter 3, we reported the critical role played by enhanced glycolysis in house dust mite-induced allergic airways disease, an asthma model that we used in our studies. We additionally described IL1B signaling as the driver for the aforementioned glycolytic reprogramming and demonstrated its contribution to the pro-inflammatory responses in epithelial cells. Moreover, we confirmed our results in human samples where we showed that both sputum and nasal cells derived from asthmatics exhibited higher lactate levels, indicative of elevated glycolysis, compared to healthy controls. This higher lactate levels correlated positively with IL1B but negatively with lung function. We therefore suggested the potential use of elevated lactate and IL1B as non-invasive biomarkers in asthmatics.

Results from chapter 3 demonstrated increases in expression of PKM2, a key enzyme in glycolysis pathway, levels in nasal epithelial cells derived from asthmatics as well as in mice with HDM-induced allergic airways disease. Therefore, in chapter 4, we designed our experiments to study a potential role played by PKM2 in asthma pathogenesis. Here, we showed that PKM2 indeed promoted the allergic response in HDM-induced allergic airways disease and in epithelial cells, contributing to the disease pathogenesis. In addition, activating the PKM2 tetramer, using TEPP46, decreased mucus metaplasia, attenuated collagen deposition, and dampened IL-1Binduced proinflammatory cytokines in mice. As a result, we highlight the possibility that targeting PKM2 might be of significant value in asthma therapeutics. 
After confirming the involvement of metabolic alterations in the development and pathogenesis of allergic airways disease, we moved to investigate the involvement of redox perturbations in this regulation. Utilizing an unbiased multi-omics approach in chapter $\mathbf{5}$, we first confirmed the IL1B contribution to the enhanced glycolysis. Secondly, we demonstrated a role played by S-glutathionylation chemistry in regulating glycolysis in epithelial cells. This result stemmed from the correlation between overall increases in S-glutathionylation and elevated glycolysis. In addition, multiple glycolytic genes were targets for S-glutathionylation, further linking metabolic reprogramming with redox biochemistry. Interestingly, we also showed the impact of the higher S-glutathionylation on inflammatory signal in epithelial cells, notably the increases in TSLP and GM-CSF. This further emphasize the link between redox perturbations and inflammatory cytokines in asthma.

Our multi-omics analysis in chapter 5 unraveled an interesting observation whereby GSH, the mainstay of redox homeostasis, is increased in response to IL1B. In chapter $\mathbf{6}$, we sought to better understand the molecular mechanism underlying this GSH upregulation and whether this have any impact on IL1B-induced inflammatory signaling and therefore is relevant to asthma. In this chapter we identified a new mechanism by which GSH, through S-glutathionylation of the deubiquitinase OTUB-1, positively regulated its own synthesis. This mechanism involved the upregulation of system $\mathrm{x}_{c}^{-}$, a cystine transporter that have implications in multiple diseases. In addition, we demonstrated the contribution of GSH (and system $x_{c}^{-}$) in IL1B-induced proinflammatory responses, specifically TSLP. The outcome of this chapter highlights the role played by S-glutathionylation biochemistry in metabolic reprogramming and its contribution to inflammatory events. In addition, in this chapter we identify a new paradigm important in GSH regulation that may translate to better therapeutics in multiple inflammatory conditions involving GSH, including asthma.

In chapter 7, we discussed the main findings of the thesis and elaborated on connecting the dots between metabolic reprogramming, redox homeostasis and their relevance in asthma pathogenesis. Overall, work in this thesis broaden our current knowledge on the crosstalk between glycolytic reprogramming and redox perturbation and their implications in asthma pathogenesis 
CHAPTER 9: IMPACT 


\section{Background and rationale}

Metabolism is the term used to describe the chemical processes taking place in our bodies to generate energy and supply our cellular needs. Any alterations in these reactions whether to support increase energy demand or other processes such as growth or proliferation will fall under metabolic reprogramming [1]. Originally described in cancer cells to support tumor growth under harsh conditions, invasion and metastasis, the term metabolic reprogramming now is not confined to cancer [2]. In fact, metabolic reprogramming has been linked to many diseases and syndromes including diabetes, amyotrophic lateral sclerosis, obesity, and chronic obstructive pulmonary disease [3-6]. Glycolysis can simply be defined as the breakdown of the sugar (glucose) in several steps and by multiple enzymes to generate energy. It is one of the earliest and most studied metabolic pathways that impact our daily cellular functions.

Oxidants are normal byproducts of multiple cellular processes that were originally thought to always be harmful. However, in the recent century, numerous studies have proved otherwise, and now oxidants are known to have several roles in physiological processes including wound healing and fighting pathogens [7, 8]. In addition, oxidants serve as important regulators of many cellular processes. One mechanism by which oxidants work is through oxidizing their target proteins, changing their structure and function. However, too much oxidants will indeed be harmful, and in order to prevent damage from these excessive amounts of oxidants, our bodies adapt several ways to combat them. The term redox homeostasis then appeared to describe the balance between the reactive oxidant species (ROS) production and removal. Scientists have identified links between glycolytic reprogramming (changes in the glycolysis pathway) and redox homeostasis, whereby the sugar breakdown provides molecules (NADPH) necessary for synthesizing glutathione (GSH), a major determinant of redox homeostasis [9]. Similarly to metabolic reprogramming, redox homeostasis has also been described to contribute to many diseases including cancer and several inflammatory conditions [10, 11].

Asthma is a very common lung disease that affects more than 300 million people around the globe. The disease has drastic impact on patients' quality of lives and economies [12, 13]. Typical symptoms of asthma include shortness of breath, wheezing, cough, chest tightness accompanied with reversible airway blockade. Airway chronic inflammation, aifflow obstruction, airway hyperresponsiveness (AHR) and remodeling are considered the characteristics of asthma, however the disease varies a lot between individuals. Several genetic and physiological factors are behind the variability and severity of the symptoms in different patients [13]. Therefore, scientists and clinicians have divided asthma into several subtypes to help group together patients with similar clinical symptoms (phenotypes) or same underlying mechanism (endotypes). This 
aims at tailoring treatment strategies with the goal of improving patients' clinical symptoms and quality of lives. Unfortunately, due to asthma complexity, there is a huge overlap between these different subtypes that makes it very challenging to subcategorize all asthma patients. Additionally, most patients who suffer from the classical asthma eosinophilic inflammation are responsive to corticosteroid treatments. However, a substantial group of these patients do not respond to treatments, or display a neutrophilic inflammation with severe disease [14]. Therefore, a better understanding of the molecular mechanism/s underlying asthma is needed. In addition, the identification of new biomarkers is very critical to benefit this subgroup of patients with no specific asthma subtype and are not responsive to the current therapies. In this work, we aim at understanding the molecular mechanism underlying asthma, and specifically investigating a potential role played by glycolytic reprogramming and redox homeostasis in the development and progression of the disease.

\section{Findings:}

As stated earlier, in this thesis, our first aim was to study if metabolic reprogramming contributes to pro-inflammatory signaling in epithelial cells and whether this affects the pathogenesis of allergic airways disease and asthma. Here, we demonstrate that indeed enhanced glycolysis is a major contributor to the inflammatory response and worsening lung function in asthma models. Specifically, we show that the key inflammatory mediator, IL1B, is responsible for the increased glycolysis identified in our analysis. Importantly, we detected higher levels of IL1B in samples derived from asthma patients compared to healthy controls. These elevated IL1B levels positively correlate with higher amounts of lactate, used as a marker for enhanced glycolysis. Interestingly, this correlation was found solely in patients with neutrophilic asthma, which is a severe subtype that does not usually respond to treatments. Moreover, we show that IL1B signaling induces the release of the pro-inflammatory cytokine TSLP from epithelial cells, which has also been linked with severe asthma. The contribution of the glycolytic enzyme PKM2 in asthma pathogenesis further confirm the involvement of glycolytic reprogramming in the development of the disease. Remarkably, we show the advantages of activating PKM2 that lowers mucus metaplasia, collagen deposition and inflammation.

Our second main objective in this thesis was to investigate if redox perturbations affect asthma pathogenesis. Our results in epithelial cells highlight multiple pathways that are regulated by redox homeostasis including metabolic pathways, glycolysis, and biosynthesis of amino acids. Interestingly, we also show that glycolysis is one of the pathways that is extensively regulated by S-glutathionylation, an important oxidation process of reactive cysteines. This conclusion is based 
on the enhanced glycolysis detected in settings of increased S-glutathionylation, as well as the identification of multiple glycolytic intermediates being targets for S-glutathionylation. Importantly, TSLP and GM-CSF, two pro-inflammatory cytokines relevant in asthma, were found to be increased in settings of increased S-glutathionylation. These data point at a role played by Sglutathionylation and redox imbalance in regulating glycolytic reprogramming, inflammation, and contribution in asthma pathogenesis.

Our last aim was to unravel the mechanism by which IL1B and/or enhanced Sglutathionylation increases GSH, the mainstay of redox homeostasis, levels. In here, we show that IL1B stabilizes system $x_{c}^{-}$, a key determinant in GSH homeostasis, in an SLC7A11/ OTUB1SSG- dependent manner. This highlights a new unique regulatory mechanism by which GSH, through S-glutathionylation, regulates its own synthesis. Finally, we demonstrate that lowering GSH levels (by the inhibition of its rate limiting synthesizing enzyme $\mathrm{GCL}$ or system $\mathrm{x}_{\mathrm{c}}^{-}$) attenuates TSLP release from lung epithelial cells, suggesting GSH relevance in several inflammatory disorders including asthma.

\section{Relevance, direct impact and target groups:}

Results in this thesis broaden our current understanding on mechanisms underlying asthma pathogenesis. Herein, we demonstrate that glycolytic reprogramming and redox perturbations are both contributors to the disease development. In addition, we propose increases in IL1B and lactate as potential non-invasive and rapid biomarkers in asthma, specifically in the neutrophilic subtype. These biomarkers will help in better characterization of these patients and categorize them into their respective asthma phenotype and therefore will aid with their treatment strategy. In addition, we show that inhibiting IL1 signaling attenuates pro-inflammatory cytokines. IL1 receptor blocker, anakinra, is an FDA approved drug already in the market for treatment of rheumatoid arthritis. Our data suggest that patients with severe asthma subtypes may benefit from the use of this drug. Beyond asthma, elevated IL1B has been linked to multiple diseases including diabetes, stroke, and Alzheimer's disease [15-17]. Strikingly, COVID-19 patients exhibit higher levels of lactate and PKM2, as well as elevated circulating and plasma IL1B levels in severe cases $[18,19]$. Therefore, it is worthwhile to test effect of neutralizing IL1B signaling in these patients.

In addition, the new SLC7A11/ OTUB1-SSG mechanism of GSH regulation proposed here is enlightening to the redox field and will have a great impact on multiple diseases in which GSH is increased including asthma. Some aggressive cancer subtypes have upregulated SLC7A11 and/or OTUB1 and this is usually accompanied by elevated levels of GSH. Our results may then 
explain part of the mechanistic details underlying these cancers and help tailor better therapeutics. Furthermore, the effect of GSH inhibition on attenuating pro-inflammatory cytokines in epithelial cells point at GSH being a potential regulator of inflammatory diseases such as asthma. Importantly, these results change the public notion that antioxidants are always good to better understand the fine balance between oxidants and antioxidants.

Moreover, herein we demonstrated the beneficial effects of activating the glycolytic enzyme PKM2 in lowering inflammation and asthma. This result may translate into developing PKM2 activators that will not only benefit asthma patients but also patients with other inflammatory conditions as well. Together all the above observations highlight the relevance of our work and broaden its implications beyond asthma.

Results presented herein are (and will be) published in reputable scientific journals and will broaden the knowledge of the research and academic field, in addition to gaining the interest of the pharmaceutical industry. However, it is also important that this work reach more of the general public outside of the scientific field. Therefore, we strongly recommend simplifying the scientific findings and presenting them in the forms of articles in newspapers, magazines, social media, and videos for easier access by the general population. Additionally, a clear detailed communication between scientists, health care professionals and their patients/ their families is a must to increase awareness of ongoing research and new approaches to combat disorders and diseases. 


\section{$\underline{\text { References: }}$}

1. Ward, P.S. and C.B. Thompson, Metabolic reprogramming: a cancer hallmark even warburg did not anticipate. Cancer Cell, 2012. 21(3): p. 297-308.

2. Marelli-Berg, F.M., H. Fu, and C. Mauro, Molecular mechanisms of metabolic reprogramming in proliferating cells: implications for T-cell-mediated immunity. Immunology, 2012. 136(4): p. 3639.

3. Martin, S.D. and S.L. McGee, Metabolic reprogramming in type 2 diabetes and the development of breast cancer. J Endocrinol, 2018. 237(2): p. R35-r46.

4. Szelechowski, M., et al., Metabolic Reprogramming in Amyotrophic Lateral Sclerosis. Sci Rep, 2018. 8(1): p. 3953.

5. Michelet, X., et al., Metabolic reprogramming of natural killer cells in obesity limits antitumor responses. Nat Immunol, 2018. 19(12): p. 1330-1340.

6. Zhao, H., P.A. Dennery, and H. Yao, Metabolic reprogramming in the pathogenesis of chronic lung diseases, including BPD, COPD, and pulmonary fibrosis. Am J Physiol Lung Cell Mol Physiol, 2018. 314(4): p. L544-I554.

7. Sen, C.K., et al., Oxygen, oxidants, and antioxidants in wound healing: an emerging paradigm. Ann N Y Acad Sci, 2002. 957: p. 239-49.

8. Peterhans, E., Oxidants and antioxidants in viral diseases: disease mechanisms and metabolic regulation. J Nutr, 1997. 127(5 Suppl): p. 962s-965s.

9. Obrosova, l., et al., Glycolytic pathway, redox state of $N A D(P)$-couples and energy metabolism in lens in galactose-fed rats: effect of an aldose reductase inhibitor. Curr Eye Res, 1997. 16(1): p. 34-43.

10. Ghoneum, A., et al., Redox Homeostasis and Metabolism in Cancer: A Complex Mechanism and Potential Targeted Therapeutics. Int J Mol Sci, 2020. 21(9).

11. Bekeschus, S., et al., Oxidants and Redox Signaling: Perspectives in Cancer Therapy, Inflammation, and Plasma Medicine. Oxid Med Cell Longev, 2017. 2017: p. 4020253.

12. John, J., et al., Regional Disparity in Asthma Prevalence and Distribution of Asthma Education Programs in Texas. J Environ Public Health, 2020. 2020: p. 9498124.

13. Dharmage, S.C., J.L. Perret, and A. Custovic, Epidemiology of Asthma in Children and Adults. Front Pediatr, 2019. 7: p. 246.

14. Kuruvilla, M.E., F.E. Lee, and G.B. Lee, Understanding Asthma Phenotypes, Endotypes, and Mechanisms of Disease. Clin Rev Allergy Immunol, 2019. 56(2): p. 219-233.

15. Dinarello, C.A., M.Y. Donath, and T. Mandrup-Poulsen, Role of IL-1beta in type 2 diabetes. Curr Opin Endocrinol Diabetes Obes, 2010. 17(4): p. 314-21.

16. Murray, K.N., A.R. Parry-Jones, and S.M. Allan, Interleukin-1 and acute brain injury. Front Cell Neurosci, 2015. 9: p. 18.

17. Shaftel, S.S., W.S. Griffin, and M.K. O'Banion, The role of interleukin-1 in neuroinflammation and Alzheimer disease: an evolving perspective. J Neuroinflammation, 2008. 5: p. 7.

18. McElvaney, O.J., et al., Characterization of the Inflammatory Response to Severe COVID-19 IIlness. Am J Respir Crit Care Med, 2020. 202(6): p. 812-821.

19. Szarpak, L., et al., Lactate dehydrogenase level as a COVID-19 severity marker. Am J Emerg Med, 2021. 45: p. 638-639. 



\section{ACKNOWLEDGMENTS}


The journey of obtaining Ph.D. is an immense undertaking that spans many years. There are numerous people to acknowledge and be grateful for and I would like to start with thanking my promotion team.

Professor Wouters, Yvonne, and Niki, thank you very much for all the efforts you put to make my dream come true. You guided me through my project, answered my questions, provided great feedback, and made insightful contributions to my work. Yvonne, you believed in me and my abilities to obtain a $\mathrm{PhD}$, convinced me to start, always pushed me to do my best and above all gave me the opportunity to work and learn in your lab. Your enthusiasm and passion to science always amazed and inspired me. Your door was always open to my endless questions. Our long discussions and meetings have taught me how to critic my own work and sharpened my thoughts. Thank you Yvonne for all of this, and I am looking forward to our work in the future.

I was also fortunate to work in a friendly and caring environment in the Janssen-Heininger's lab. I would like to thank Dr. Jos van der Velden not only for his valuable scientific input but also for keeping the lab fun. Our daily morning chats about everything, coffee and water morning walks and random jokes here and there made my time in the lab more fun. To my bench mate for many years, Karrie Lahue, and friend, Wyatt Chia, I miss working with both of you. You were so kind to help me find my ways in the lab when I first started, taught me many techniques, covered me on the weekends, and much more. I already miss our long chats, lunches, Karrie's brownies, Wyatt's baking trials, dinners, language lessons, and fun outside the lab. I am also very thankful for our previous lab members: Xi Qian, Bob Schneider, Allison Manuel, Evan Elko, Cheryl Van de Wetering and Dylan Casey for the many things we enjoyed together. Joe Druso, I always enjoyed asking you questions because of the way you simplify and explain things. I admire your patience and passion for teaching and I believe you will affect many students' lives if you decide to follow this path. Liz Corteselli, I enjoyed working with you very much and thank you for your input on my work. Cuixia Erickson, my bench buddy, you do a great work in the lab (upstairs and downstairs), keep it up my friend! Oh, and do not stop getting me chocolates and sweets! Zhihua Peng, I sincerely thank you for your help with the CRISPR design. This was a nightmare for me, but you made it more like a dream. I promise one day I will learn skiing and make you proud. Max Macpherson, thank you for keeping up with me the last couple years. You were always ready to answer my early morning emails, help me with the enormous amount of recombinant protein I ask for, sequence and check plasmids quality controls and much more. 
To my friends here in Vermont, a BIG THANK YOU! You always listened to me with passion and made my work sounds exciting, even in the times that I was down. You forgave me for the many many times I had to cancel our gatherings because I have an experiment going on, or when I needed to feed my cells. You always had arms open when I needed to vent about things not working the way they should. You helped taking care of the kids when I needed to go to work during school and daycare breaks. I feel I have a family here in Vermont because of you all. Friends in Egypt, USA, Canada and other countries, you also supported me, made me laugh, and gave me hope. I miss you all and hopefully we will see each other soon.

To my beloved Osama, we started our journey together 14 years ago, and I wouldn't change one single thing about it. Thank you for believing in me, comforting me, encouraging me, making me laugh, and holding me tight. I would have never accomplished this without you. Hamza and Karim, words are not enough to express how much love and pride I carry for the two of you. I apologize if I was ever too busy to be the good mom you needed, but know that I will always be here when you need me, no matter how busy I seem.

$$
\begin{aligned}
& \text { إلى أغلى الناس، إلى أبى وأمى: أهديكما هذه الرسالة اليوم وأتمنى أن تكونا فخورين بأبنتكما. حقاً أنا لا أجد كلمات تكفي لوصف كل }
\end{aligned}
$$

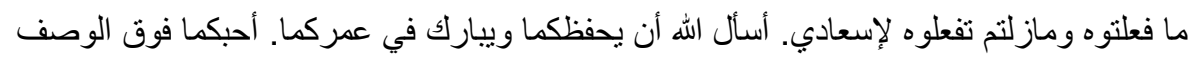

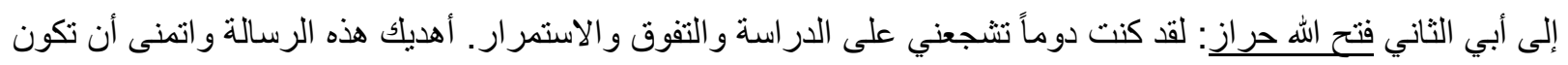

$$
\begin{aligned}
& \text { فخوراً بي. أفتقدك كثير اً، رحمك الله يا حبيبي. } \\
& \text { إلى أمي الغالية إيفاء: شكر ا على كل شيء، على الدعم و الدعاء والحب. أتمنى وجودك بجانبي. } \\
& \text { هيثُ، مريم، أمانى، محمود، و الأو لاد: عائلتي و أحبائي شكر ا لكم، أفتقدكم كثير اً. }
\end{aligned}
$$




\section{List of Publications and Curriculum Vitae}




\section{List of Publications:}

1. Aboushousha R, van der Velden JL, Peng Z, Chia SB, Manuel AM, MacPherson M, Erickson C, Reisz JA, D’Alessandro A, Wouters EFM, Reynaert NL, Janssen-Heininger YMW. (2021) Glutaredoxin controls glutathione levels via deglutathionylation of ovarian tumor ubiquitin aldehyde binding 1 and stabilization of system XC. To be submitted to Cell Metabolism

2. van de Wetering $C$, Manuel AM, Sharafi M, Aboushousha R, Qian X, Erickson C, MacPherson M, Chan G, Adcock I, ZounematKermani N, Schleich F, Louis R, Bohrnsen E, D'Alessandro A, Wouters EF, Reynaert NL, Li J, Wolf CR, Henderson CJ, Lundblad LKA, Poynter ME, Dixone AE, Irvin CG, van der Vliet A, van der Velden JL, JanssenHeininger YMW. (2021) Glutathione-S-transferase P promotes glycolysis in asthma in association with oxidation of pyruvate kinase M2. Redox Biol. 47:102160

3. Manuel $\mathrm{AM}^{*}$, van de Wetering $\mathrm{C}^{*}$, van der Velden JL, Aboushousha R, MacPherson $\mathrm{M}$, Erickson C, Poynter ME, Irvin CG, Taatjes DJ, Dixone AE, van der Vliet A, Anathy V, Janssen-Heininger YMW. (2021) Dimethyl Fumarate Attenuates Allergic Airways Inflammation in Mice in Association with Decreases in Glycolytic Reprogramming. Am J Physiol Lung Cell Mol Physiol. In revision

4. Aboushousha $\mathbf{R}^{*}$, Elko $E^{*}$, Chia SB, Manuel AM, van de Wetering $C$, van der Velden JL, MacPherson M, Erickson C, Reisz JA, D'Alessandro A, Wouters EFM, Reynaert NL, Lam YW, Anathy V, van der Vliet A, Seward DJ, Janssen-Heininger YMW. (2021) Glutathionylation chemistry promotes interleukin 1 beta-mediated metabolic reprogramming and pro-inflammatory signaling in lung epithelial cells. FASEB $\boldsymbol{J}$. 35:e21525 * Co-first authors.

5. Manuel AM, van de Wetering $C$, MacPherson M, Erickson $C$, Murray $C$, Aboushousha $\underline{\mathbf{R}}$, van der Velden JL, Dixon AE, Poynter ME, Irvin CG, Taatjes DJ, van der Vliet A, Anathy V, Janssen-Heininger YMW. (2021) Dysregulation of pyruvate kinase M2 promotes inflammation in murine obese allergic asthma. Am J Respir Cell Mol Biol. 64:709-721 
6. Chia SB, Nolin JD, Aboushousha R, Erickson C, Irvin CG, Poynter ME, van der Velden JL, Taatjes D, van der Vliet A, Anathy V, Janssen-Heininger YMW. (2020) Glutaredoxin deficiency promotes activation of the transforming growth factor beta pathway in airway epithelial cells, in association with fibrotic airway remodeling. Redox Biology. 37:101720

7. van de Wetering $C^{*}$, Aboushousha $\mathbf{R}^{*}$, Manuel AM, Chia SB, Erickson $C$, MacPherson MB, van der Velden JL, Anathy V, Dixon AE, Irvin CG, Poynter ME, van der Vliet A, Wouters EFM, Reynaert NL, Janssen-Heininger YMW. (2020) Pyruvate Kinase M2 Promotes Expression of Proinflammatory Mediators in House Dust Mite-Induced Allergic Airways Disease. J Immunol. 204:763-774. * Co-first authors.

8. Chia SB, Elko EA, Aboushousha R, Manuel AM, van de Wetering C, Druso JE, van der Velden J, Seward DJ, Anathy V, Irvin CG, Lam YW, van der Vliet A, Janssen-Heininger $\mathrm{Y}$. (2019) Dysregulation of the glutaredoxin/S-glutathionylation redox axis in lung diseases. Am J Physiol Cell Physiol. 318:C304-C327.

9. Basso L, Aboushousha R, Fan CY, Iftinca M, Melo H, Flynn R, Agosti F, Hollenberg MD, Thompson RJ, Bourinet E, Trang T, Altier C. (2019) TRPV1 regulates opioid analgesia during inflammation. Sci Signaling. 12:eaav0711.

10. Elko EA, Cunniff B, Seward DJ, Chia SB, Aboushousha R, van de Wetering $C$, van der Velden J, Manuel A, Shukla A, Heintz NH, Anathy V, van der Vliet A, Janssen-Heininger YMW. (2019) Peroxiredoxins and Beyond; Redox Systems Regulating Lung Physiology and Disease. Antioxid Redox Signal. 31:1070-1091.

11. Anathy V, Lahue KG, Chapman DG, Chia SB, Casey DT, Aboushousha R, van der Velden JLJ, Elko E, Hoffman SM, McMillan DH, Jones JT, Nolin JD, Abdalla S, Schneider R, Seward DJ, Roberson EC, Liptak MD, Cousins ME, Butnor KJ, Taatjes DJ, Budd RC, Irvin CG, HoY-S, Hakem R, Brown KK, Matsui R, Bachschmid MM, Gomez JL, Kaminski $\mathrm{N}$, van der Vliet A, Janssen-Heininger YMW (2018) Reducing protein oxidation reverses lung fibrosis. Nature Medicine. 24:1128-1135. 
12. Qian X, Aboushousha R, van de Wetering C, Chia SB, Amiel E, Schneider RW, van der Velden JLJ, Lahue KG, Hoagland DA, Casey DT, Daphtary N, Ather JL, Randall MJ, Aliyeva M, Black KE, Chapman DG, Lundblad LKA, McMillan DH, Dixon AE, Anathy V, Irvin CG, Poynter ME, Wouters EFM, Vacek PM, Henket M, Schleich F, Louis R, van der Vliet A, Janssen-Heininger YMW (2017) IL-1/inhibitory kB kinase $\varepsilon$-induced glycolysis augments epithelial effector function and promote allergic airways disease. $\boldsymbol{J}$ Allergy Clin Immunol. 142:435-450.e10.

13. Iftinca M, Flynn R, Basso L, Melo H, Aboushousha R, Taylor L, Altier C (2016) The stress protein heat shock cognate 70 (HSC70) inhibits the Transient Receptor Potential Vanilloid type 1 (TRPV1) channel. Mol Pain. 12:1744806916663945

14. Flynn R, Chapman K, Iftinca M, Aboushousha R, Varela D, Altier C (2014) Targeting the Transient Receptor Potential Vanilloid Type 1 (TRPV1) Assembly Domain Attenuates Inflammation-induced Hypersensitivity. J Biol Chem. 289: 16675-87. 


\section{CURRICULUM VITAE}

Reem Aboushousha was born on August 15, 1986, in Alexandria, Egypt. She finished her secondary school diploma in 2003 with A+ grade. She then joined Faculty of Pharmacy, Alexandria University, in Egypt in 2003 and obtained her bachelor's degree in Pharmaceutical Sciences in 2008. She worked as a community pharmacist until 2010 in Alexandria, Egypt. During the same period, she joined staff at Faculty of Pharmacy and worked as a teaching assistant of biochemistry for clinical pharmacy students. In 2010, she moved to Canada with her husband and decided to start her career in science. She joined Dr. Christophe Altier's laboratory at the University of Calgary, Cumming School of Medicine, as a graduate student in 2013. During her Master studies, Reem's passion for science grew and she received multiple honorary awards, including the Queen Elizabeth Award and the Alberta Graduate Student Scholarship. She obtained her master's degree in Medical Sciences in 2015. In September 2015, she joined Dr. Yvonne Janssen-Heininger's laboratory at the University of Vermont as a research technician before deciding to join the PhD program in Maastricht under the supervision of Prof. Dr. E.F.M. Wouters, Prof. Dr. Y.M.W. Janssen-Heininger and Dr. N.L. Reynaert. Her PhD project focused on studying metabolic reprogramming and redox regulation in lung diseases. Work from her PhD has been published in several manuscripts as described in this thesis. Reem plan to continue a scientific career at the University of Vermont after obtaining her PhD. 